University of Louisville

ThinkIR: The University of Louisville's Institutional Repository

$5-2020$

\title{
A novel approach to HIV prevention: understanding multi-level influences on HIV pre-exposure prophylaxis (PREP) uptake and outreach among African Americans.
}

Suur Debrah Ayangeakaa

University of Louisville

Follow this and additional works at: https://ir.library.louisville.edu/etd

Part of the Community Health and Preventive Medicine Commons, and the Public Health Education and Promotion Commons

\section{Recommended Citation}

Ayangeakaa, Suur Debrah, "A novel approach to HIV prevention: understanding multi-level influences on HIV pre-exposure prophylaxis (PREP) uptake and outreach among African Americans." (2020). Electronic Theses and Dissertations. Paper 3367.

https://doi.org/10.18297/etd/3367

This Doctoral Dissertation is brought to you for free and open access by ThinkIR: The University of Louisville's Institutional Repository. It has been accepted for inclusion in Electronic Theses and Dissertations by an authorized administrator of ThinkIR: The University of Louisville's Institutional Repository. This title appears here courtesy of the author, who has retained all other copyrights. For more information, please contact thinkir@louisville.edu. 


\title{
A NOVEL APPROACH TO HIV PREVENTION: UNDERSTANDING MULTI- LEVEL INFLUENCES ON HIV PRE-EXPOSURE PROPHYLAXIS (PREP) UPTAKE AND OUTREACH AMONG AFRICAN AMERICANS
}

\author{
By \\ Suur Debrah Ayangeakaa \\ MPH, Chicago State University, May 2013 \\ B.A., Dordt College, May 2009

\begin{abstract}
A Dissertation
Submitted to the Faculty of the in Partial Fulfillment of the Requirements

for the Degree of

Doctor of Philosophy

in Public Health Sciences

Department of Health Promotion and Behavioral Sciences

University of Louisville

Louisville, Kentucky
\end{abstract} \\ School of Public Health and Information Sciences of the University of Louisville
}

May 2020 
Copyright $^{\circledR} 2020$ by Suur Debrah Ayangeakaa

All rights reserved 



\title{
A NOVEL APPROACH TO HIV PREVENTION: UNDERSTANDING MULTI- LEVEL INFLUENCES ON HIV PRE-EXPOSURE PROPHYLAXIS (PREP) UPTAKE AND OUTREACH AMONG AFRICAN AMERICANS
}

\author{
By \\ Suur Debrah Ayangeakaa \\ MPH, Chicago State University, May 2013 \\ B.A., Dordt College, May 2009
}

Dissertation Approved on

April 30, 2020

by the following Dissertation Committee

Jelani Kerr, PhD, MSPH - Dissertation Chair

Ryan Combs, $\mathrm{PhD}$, MA

Lesley Harris, MSW, PhD

Kimberly Parker, PhD, MPH, MCHES 


\section{DEDICATION}

This dissertation is dedicated to my family.

To

My Dad, Mr. Levi O’bem Yakubu, my Mom, Dr. Angell Nguemo Yakubu for all your countless years of sacrifice and perseverance against all odds to give me and my siblings a solid foundation and a bright future, for believing in me, and for investing in my education.

To

To my American host Family, Dad, Marty Ozinga (III) and Mom Ruth Ozinga (of blessed memory). Your love, prayers, and financial support made the furthering of my education possible. I wish mom were here to see this; she would be so proud of me. To

My husband, Dr. Akaa Daniel Ayangeakaa. This PhD was your idea. You believed I could do it.

To

My two lovely children, Anna and Josiah. You endured so much. You are troupers 


\section{ACKNOWLEDGEMENTS}

First and foremost, my most sincere appreciation goes to God Almighty for his love and faithfulness towards me. Lord knows, were it not for my faith in God, I would not be here today, and I would most certainly not have completed this $\mathrm{PhD}$ program. Many odds were stacked against me, but with the help of God, I prevailed. With God's guidance and the help He provided through a supportive spouse, family, friends, and an incredibly empathetic graduate school environment at the U of L School of Public Health and Information Sciences (SPHIS), I was able to stay the course and run the race to the finish line. I owe you my life, dear heavenly Father. To you be all the glory, honor, and adoration. You reign forever!

This dissertation would not be possible without the support of my committee. I want to thank Dr. Jelani C. Kerr (my dissertation chair), Dr. Ryan Combs, Dr. Lesley Harris, and Dr. Kimberly Parker for taking the time to help me finetune the conceptualization of this dissertation, for reading, reviewing, and editing this dissertation and the other papers we have worked on together. Thank you for your valuable insight and feedback to ensure that this work meets the standard for a successful completion of the doctoral program in public health. I want to specially thank my dissertation chair and dedicated mentor, Dr. Kerr. You went above and beyond for me. Thank you for believing in me and for investing your time and resources that afforded me the requisite preparation and support during my doctoral studies. Through your endearing and professional demeanor, along with your consistent display of exemplary leadership qualities, you 
instilled in me the level of discipline, sound judgment, goal orientation, and resultsdriven mentality needed to successfully complete this doctoral program. I learned a great deal from working with you on multiple research projects from inception to dissemination during the course of my studies, an experience that will no doubt contribute to my success in the field of public health and beyond. Thank you!

My heartfelt gratitude goes to Dr. Combs for welcoming me into your research group and for giving me the chance to receive hands-on training and to develop field expertise in qualitative research approaches under your leadership. That experience greatly influenced my decision to ultimately pursue a purely qualitative dissertation. I am very grateful. To Dr. Lesley Harris, I sincerely appreciate you investing so much of your time and effort into helping me further sharpen and solidify my qualitative research skills (particularly the grounded theory approach) that formed the basis of my entire dissertation. Your consistent mentorship and tutelage ensured that I could take on such a dauting task and execute it with confidence. Thank you! To Dr. Kimberly Parker, you have been a true inspiration and an encouragement. Your mentorship (especially your line of questioning) spurred me to think outside the box and to envision myself in the real world effecting real change and impacting real world problems as a research expert. Thank you! I am excitedly looking forward to future collaborations.

To my participants in the AFYA study as well as the ASO project from Louisville, KY and across the U.S., thank you for sharing your expertise and experiences with me. Without your voices, this dissertation project would not be complete. I sincerely appreciate you all! 
A special thank you goes to the Department of Health Promotion and Behavioral Sciences, Dr. Monica Wendel, the current Chair, and Dr. Richard Wilson, the former Chair, for being highly supportive and accommodating of me throughout this process. Dr. Wendel, because of your philosophy of science class, I came to enjoy and appreciate qualitative research so much so that my dissertation process was truly enjoyable. I owe you my thanks! I also sincerely appreciate all of my professors who taught and mentored me at various stages of the program, Dr. Muriel Harris, Dr. Scott LaJoie, Dr. Anne Wallis, Dr. Kelly Pryor, and many others, forgive me for not mentioning all your names. I sincerely thank the entire AFYA project team, my fellow student colleagues, the support personnel, and co-researchers under Dr. Kerr's leadership, for your support during this research process. I want to specially thank Dr. Jeanelle Sears, you are a rare gem. I sincerely appreciate all your help with coding and going back and forth over multiple sessions to finalize emerging concepts and categories. I sincerely appreciate you.

I would like to thank the entire SPHIS community, faculty, staff and students alike, who impacted me in one way or another during my time at $\mathrm{U}$ of $\mathrm{L}$. A special thank you to our incredible SPHIS staff, Barbara Parker, Robin Newlon, Vikki Lewis, Wanda Long, Mellissa Schreck, Paige Wills Carol Stowers, Tammi Thomas, Tammy Albers and the entire student services clan, Jason Banta (you saved my life so many times with your stellar IT skills, Thank you!). You all touched me on a personal level and I am truly grateful for your love, smiles, small talk around the building and moral support.

I would also like to specially thank John Chenault, associate professor, Reference Department, Kornhauser Health Sciences Library for training me in endnote and literature search from the beginning of the PhD program in the Fall of 2015 and for 
continued technical support throughout my time at $\mathrm{U}$ of $\mathrm{L}$ and especially during the dissertation phase. I owe you my most sincere thanks.

To my family, my brothers, sisters, and extended family, Doom Yakubu, Chivir Ikyernum, Levi, Yakubu Jnr. (Baba), Israel Yakubu (Izzy), Jemimah Yakubu (Jaiye), Olive Yakubu, Vershima Ayangeakaa, Jennifer Okwu (Boks), Joy Tsegha, Ngufan Kwaghko. Thank you all for standing by me and for all your prayers and support. Izzy, you have called me Doc (andocu) for God knows how long, and today, your dream for me has come true. To my parents, thank you for raising me in the fear of God, for giving me a solid foundation, and implanting in me the love for education and scholarship. You taught me to reach for the sky and to never be afraid to pursue my dreams. Mummy, you were a shining example and an inspiration to me that completing a doctoral degree is possible even when you are a woman ( growing up in a culture that sometimes misunderstands and undervalues the female gender), a dedicated wife, a very busy mother (of seven), a multiple business owner and much more. You proved to me that all things are possible with God and dedication to hard work. I have been truly inspired by your exemplary lifestyle. You are an embodiment of courage, perseverance, tenacity, and resilience. It is truly a privilege to have followed in your footsteps. You encouraged us to take education seriously and to strive to positively influence society even when the odds are against us. You thought me well, mummy. Thank you! Thanks to you and daddy, I am a doctor today. I appreciate all your prayers, encouragement, and endless support throughout this difficult process.

To my incredibly intelligent and talented husband, my best friend, my confidant, my hero, my knight in shining armor, my destiny helper and super dad to our kids, the 
legendary professor of nuclear physics ( my future Nobel prize winner, as I call you), Dr. Akaa Daniel Ayangeakaa. Pursuing a PhD in Public was your idea. You are the real MVP here. When life happened and it appeared, at the time, like my dreams of becoming a medical doctor and changing the world were shattered, you reminded me of the bigger picture. You encouraged me not to give up on my passion for health care and medicine. You believed in my potential even when I was too blinded by grief and loss to see it at the time. You knew that I could do it and you encouraged me never to give up. Your unwavering support for me during this process was highly instrumental to my success. You stood by my side, endured many trials and tribulations and you sacrificed much, too much, to get me to the finish line. For that, I owe you my eternal gratitude. Thank you for being there for me and the kids, for holding the forth, for stepping up and filling the void created by my long days, weeks, and months of absences due to going back and forth between Louisville and home (Chicago, IL during the first two years of the PhD program and Annapolis, MD during the last two years). Words fail me to express how truly honored I am to have been blessed with a soul mate like you. The last 16 years (11 years of marriage and 5 years of friendship and courtship) have been the best years of my life, to say the least. I love you to life! "Thank you" is a gross understatement. I am so grateful to you for your selfless love and genuine kindness towards me. I love you so much, honey.

To my grad school bff, Dr. Baraka Muvuka. Oh my world, Baraka!!! Look how far we have both come! You were with me from the beginning of the U of L journey since we met on that faithful day during the SPHIS orientation. Throughout our program at SPHIS, we laughed, cried, played, joked around, and worked together so well. We 
were like twins (thing 1 and thing 2, like some referred to us). My dear friend, the blows on this journey were severely softened because of you. Thank you so much for being there for me. Lord knows we needed each other, and He connected us so perfectly. I am eternally grateful for our friendship and truly honored to have met you. Thanks to your beloved husband Steven Nwaokolo (SJ) too for sharing you with me all these years. I sincerely appreciate all you were and still are to me and my family. I could not have done this with without your support, dearest friend. Thank you!

How can I forget my village! To my Louisville, KY support system, Judith Civir Chia (my sister, friend, and landlady, you are a real blessing), Angela Brewer ( a rare gem and genuine human being), SirRitta Rhodes (my sister from another mother), Vitalis and Beth Lanshima (my brother and sister in-law as you are best known), Dr. Theophilus and Mrs. Taiwo OlaOlorun (my family away from family), Hurstbourne Baptist Church family, Fifth Street Baptist Church family, especially my Papa, Rev. Philip L Hodge Snr. and Mama Rev. Sharon Hodge. Thank you all so much! It really does take a village, and I would not have made it in once piece were it not for your love, prayers, encouragement, and support. Thank you all for cooking, doing laundry, providing free babysitting, letting me vent, cry, and bounce ideas off you even when you had no idea what I was saying. I sincerely appreciate all of you, and I pray that God will bless you all richly in return for your kindness towards me.

Lastly, but not the least, I would like to thank my funders, the Jewish Heritage Fund for Excellence who made this project financially possible. 


\begin{abstract}
A NOVEL APPROACH TO HIV PREVENTION: UNDERSTANDING MULTILEVEL INFLUENCES ON HIV PRE-EXPOSURE PROPHYLAXIS (PREP) UPTAKE AND OUTREACH AMONG AFRICAN AMERICANS

Suur Debrah Ayangeakaa
\end{abstract}

April 30, 2020

This dissertation examined and provided basis for addressing factors related to HIV pre-exposure prophylaxis (PrEP) engagement, outreach, and uptake among high-risk African American youth groups in Louisville, KY. This qualitative dissertation study was designed through the lens of an interpretive framework of social constructivism which holds that multiple realities and interpretations exist and are socially constructed through the lived experiences of individuals and their interactions with others. Thus, lived experiences of various African American youth groups in Louisville as well as key informants of AIDS service organizations (ASOs) across the U.S. (including Louisville) were explored to develop a deeper understanding of the barriers and facilitators to PrEP engagement, outreach, and uptake among African American youth priority groups. Seven chapters delineate the dissertation path. Chapter one provides a background and introduces the research problem and study rationale. Chapter two presents a detailed review of the literature; provides a focused overview of the epidemiologic landscape of HIV among African Americans in the U.S. as well as in Kentucky; influences on PrEP 
uptake among African Americans; and gaps in the literature. Chapter three outlines the methods utilized to answer the various research questions. Chapters four, five, and six each function as distinct manuscripts addressing the various research questions. Findings revealed multi-level/multifaceted factors influencing PrEP-use among African Americans. These include intrapersonal factors (PrEP awareness/knowledge, perceived HIV risk and PrEP need, fears/reservations about PrEP, and acceptability of PrEP), interpersonal relationships, sociocultural issues (stigma, homophobia, and homonegativity), and systemic and structural factors (such as cost of PrEP medication, insurance coverage, availability and accessibility of PrEP, and responses to PrEP engagement strategies of AIDS service organizations). Strategies and lessons learned from a national sample of ASOs informed the development of a context specific framework for successfully implementing PrEP outreach among African American groups. This dissertation addresses gaps in literature by utilizing findings to create a framework that serves as recommendations for other ASOs, including Louisville ASOs, seeking to improve PrEP service delivery and outreach among African American priority groups. 


\section{TABLE OF CONTENTS}

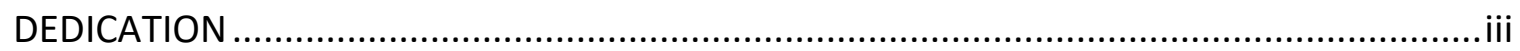

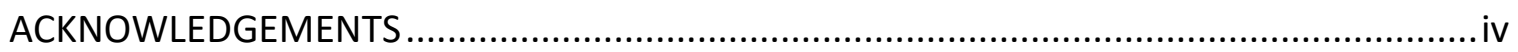

ABSTRACT

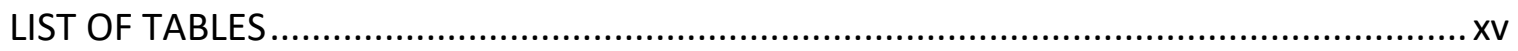

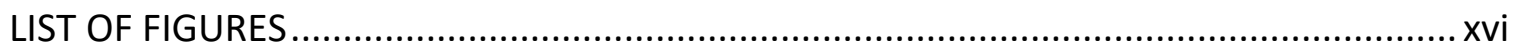

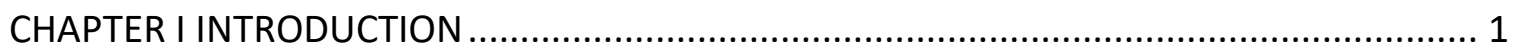

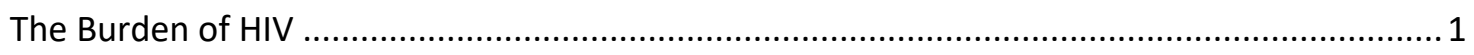

African American Sub-Groups at High Risk for HIV ...................................................... 4

Determinants of HIV Disparities among African Americans....................................................10

Examining Various HIV Prevention Approaches .............................................................11

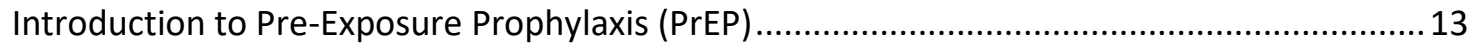

CDC Guidelines for Administering PrEP ..........................................................................15

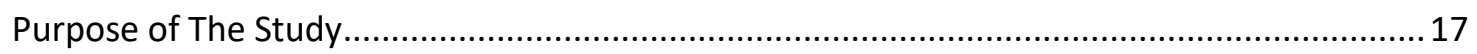

African American High-Risk Youth Groups .....................................................................19

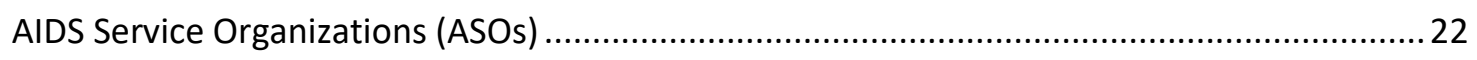

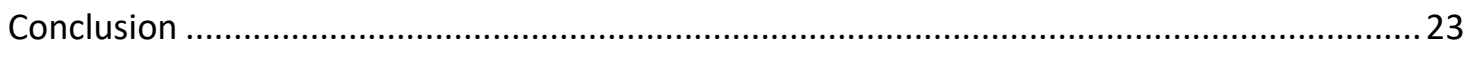

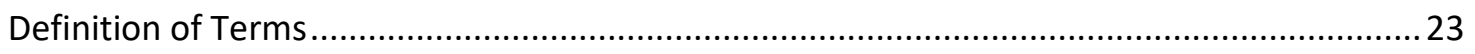

CHAPTER II LITERATURE REVIEW ..................................................................... 26

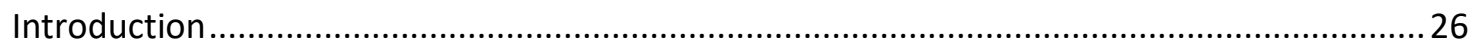

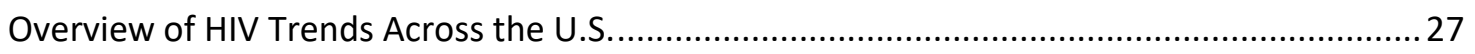

Factors Driving HIV Disparities Among African Americans..................................................30

A Brief Overview of Biomedical Interventions and Background to PrEP .................................43

Summary of PrEP Clinical Trials/Demonstration Studies ....................................................4

Examining Factors influencing PrEP Among African Americans..............................................4

AIDS Service Organizations' Role in Increasing PrEP Uptake ...................................................51 
Gaps in the Literature and Rationale for the Study........................................................53

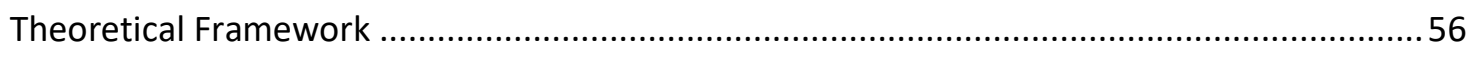

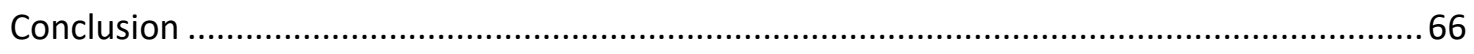

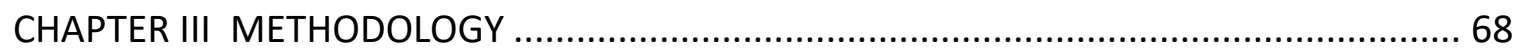

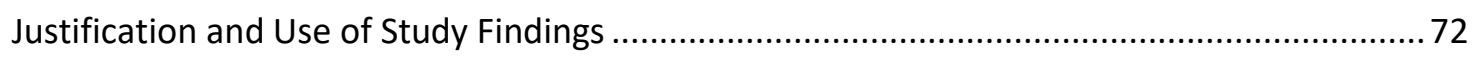

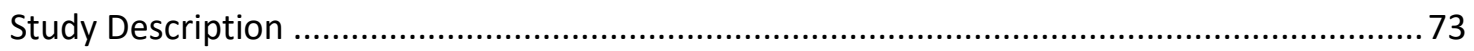

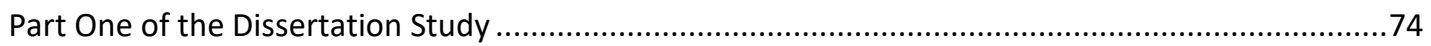

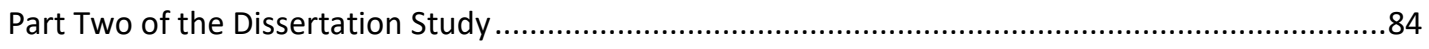

Philosophical Assumptions and Interpretive Framework .................................................99

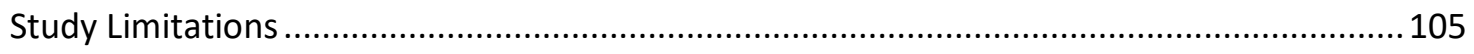

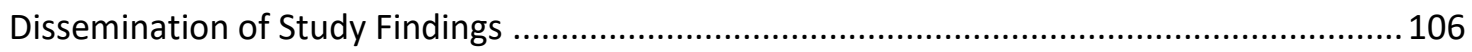

CHAPTER IV: PAPER I UNDERSTANDING INTRA-PERSONAL FACTORS IMPACTING PREEXPOSURE PROPHYLAXIS (PREP) ENGAGEMENT AMONG YOUNG AFRICAN AMERICANS

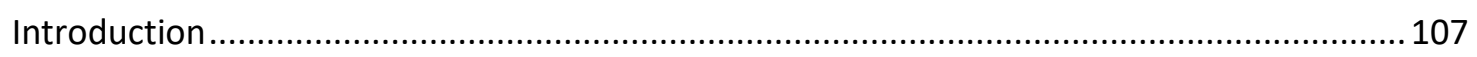

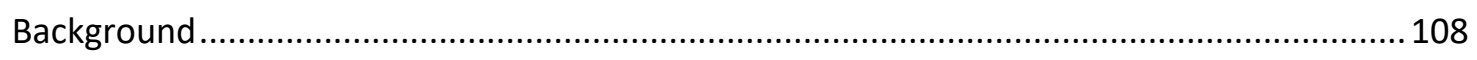

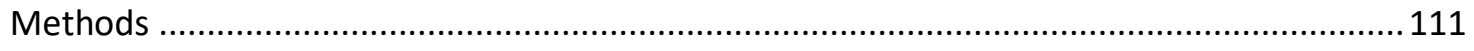

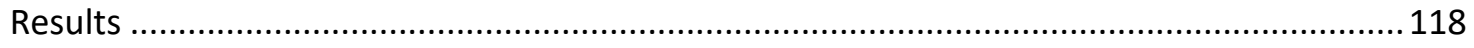

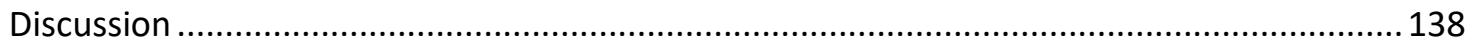

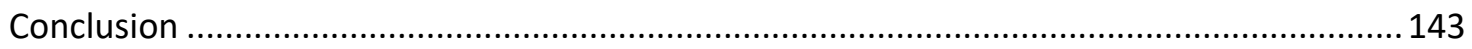

CHAPTER V: PAPER 2 BEYOND THE INDIVIDUAL: EXAMINING SOCIOECOLOGICAL AND STRUCTURAL INFLUENCES ON HIV PRE-EXPOSURE PROPHYLAXIS (PREP) ENGAGEMENT

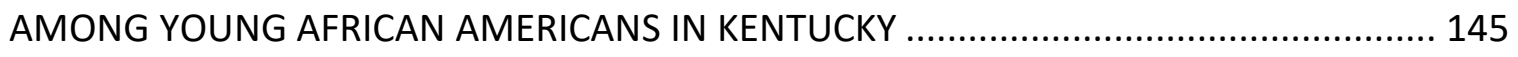

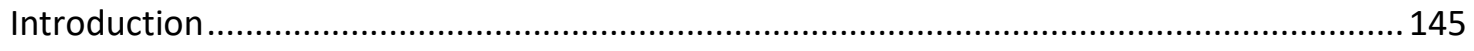

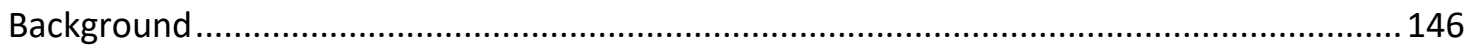

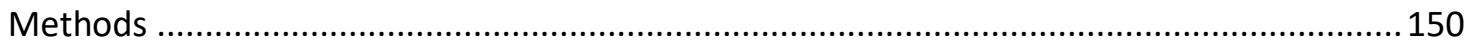

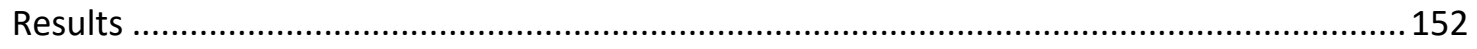

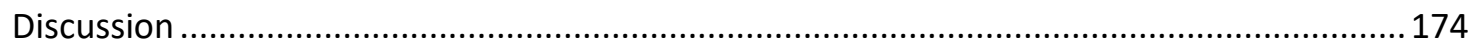

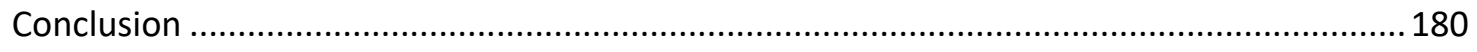

CHAPTER VI PAPER 3 "BECOMING ONE WITH THE COMMUNITY": A GROUNDED THEORY STUDY EXPLORING AIDS SERVICE ORGANIZATIONS' STRATEGIES FOR 


\section{SUCCESSFULLY IMPLEMENTING HIV PRE-EXPOSURE PROPHYLAXIS (PREP) OUTREACH}

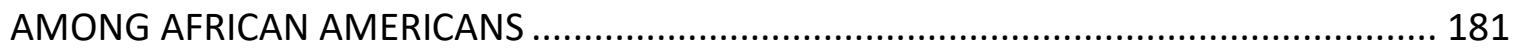

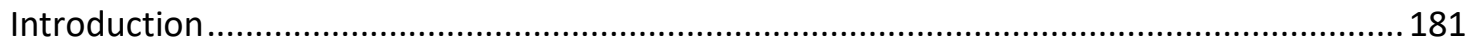

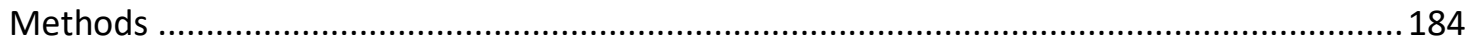

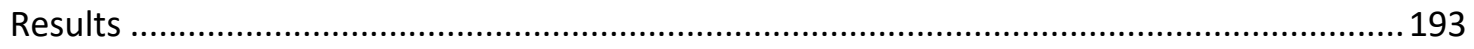

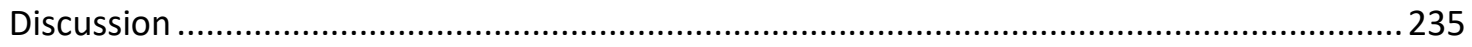

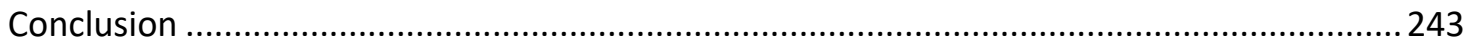

CHAPTER VII DISCUSSION, IMPLICATIONS, RECOMMENDATIONS.............................. 245

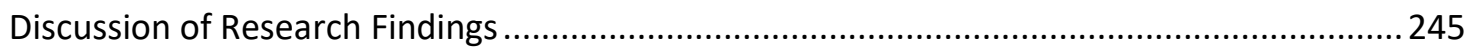

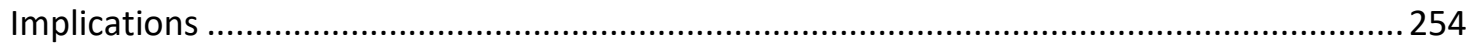

Recommendations for Implementing PrEP Engagement and Outreach among African

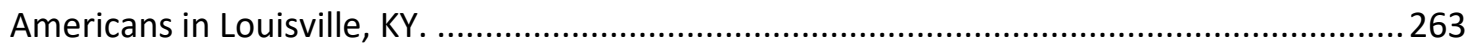

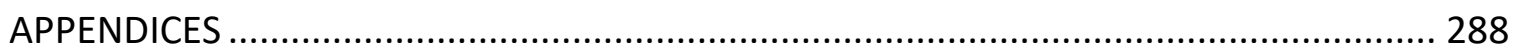

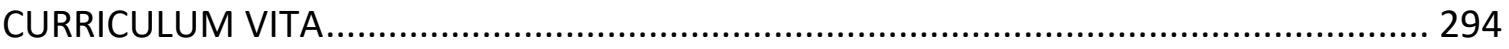




\section{LIST OF TABLES}

Table 1 National ASO Sample Study Eligibility criteria ........................................... 85

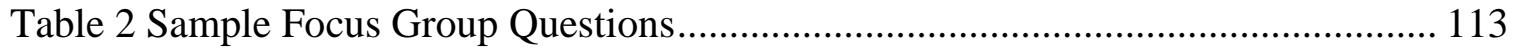

Table 3 Focus Group Sample Description. ................................................................. 117

Table 4 Characteristics of key informants from national sample of ASOs. Sample size

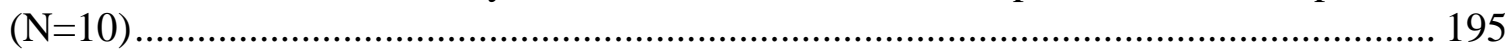

Table 5 Becoming one with the community: A Dynamic Process of ASO PrEP

Engagement and Outreach with African Americans. ............................................... 196

Table 6 Recommendations for Implementing PrEP engagement and outreach among

African Americans in Louisville, KY .......................................................................... 264 


\section{LIST OF FIGURES}

Figure 1 New HIV Cases by Race and transmission group, U.S. 2010 vs. 2016............. 2 Figure 2 HIV Cases increase among Latino and African American Gay and Bisexual Men

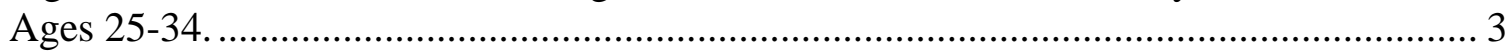

Figure 3: Annual HIV Disease Diagnosis Rates by Race/Ethnicity, Sex, Year of Diagnosis, 2011-2015, in Kentucky ......................................................................... 3

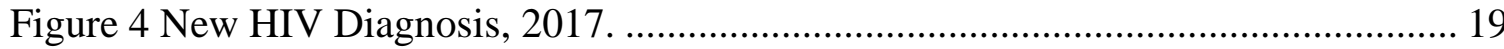

Figure 5 New Diagnosis Among Young Gay and Bisexual Men by Race/Ethnicity in the

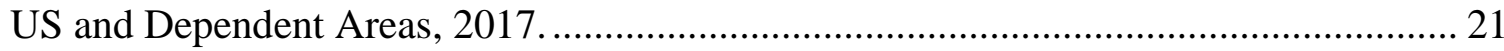

Figure 6 HIV Disease Diagnosis Rate by Age and Year of Diagnosis, 2011-2015,

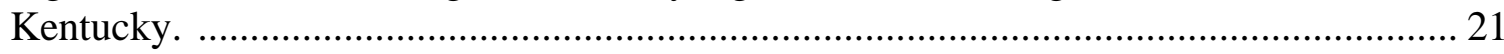

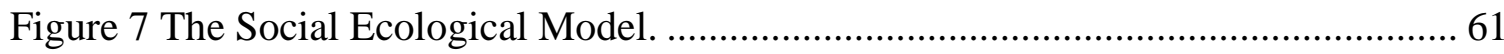

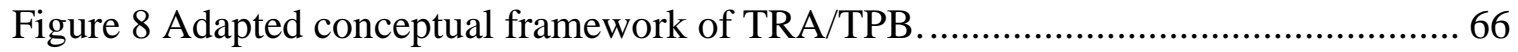

Figure 9 African American Youth Focus Groups Analysis Process.............................. 81

Figure 10 National ASO Key Informant Interviews Data Analysis process. ................. 96

Figure 11 Relationship Among Themes: Intrapersonal Factors Influencing Intention to

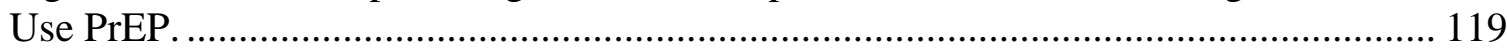

Figure 12 Facilitators or barriers to PrEP engagement and uptake among priority high

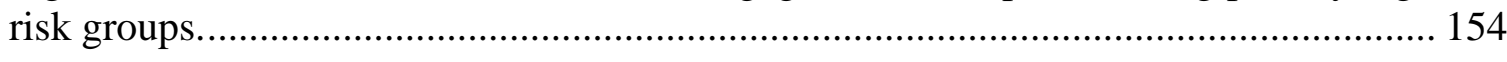

Figure 13 Becoming One with The Community: A Dynamic Process/Context Specific Framework for ASO Successful PrEP Implementation with African Americans. 197 


\section{CHAPTER I}

\section{INTRODUCTION}

\section{The Burden of HIV}

In the United States and in the state of Kentucky, (Human Immunodeficiency Virus/Acquired Immunodeficiency Syndrome ( HIV/AIDS ) disparities continue to be a major public health concern, especially among racial, sexual, gender minority groups. Although the U.S. has seen reductions in annual HIV diagnosis, some populations continue to experience disproportionately higher rates. African Americans represent one of the minority groups disproportionately impacted by HIV (Centers for Disease Control and Prevention, 2019c). While the annual HIV incidence declined over the years, rates of diagnosis either stabilized or increased for some minority groups (Figure 1) (Centers for Disease Control and Prevention, 2019c). For instance, between 2010-2016, while HIV incidence rates decreased for White gay and bisexual men, incidence increased for Hispanic gay and bisexual men, and remained stable for African American gay and bisexual men (Figure 2). The annual HIV incidence increased approximately $65 \%$ among 25-34 year old African American gay and bisexual men between 2010-2016 (Centers for Disease Control and Prevention, 2017d).

An estimated 1.1 million people are living with HIV across the U.S., and African Americans are disproportionately affected (HIV.gov, 2017). The Centers for Disease Control and Prevention (CDC) estimates that approximately $42 \%$ of all people living with 
diagnosed HIV in the U.S. by the end of 2015 were African American (Centers for Disease Control and Prevention, 2017d). They also had the highest proportion (52\%) of all deaths related to HIV disease for that year (Centers for Disease Control and Prevention, 2018b). In 2017, although African Americans only represented 13\% of the population, they accounted for the highest proportion (43\%) of all newly diagnosed HIV cases compared to any other racial group (Centers for Disease Control and Prevention, 2019b).

Figure 1 New HIV Cases by Race and transmission group, U.S. 2010 vs. 2016.

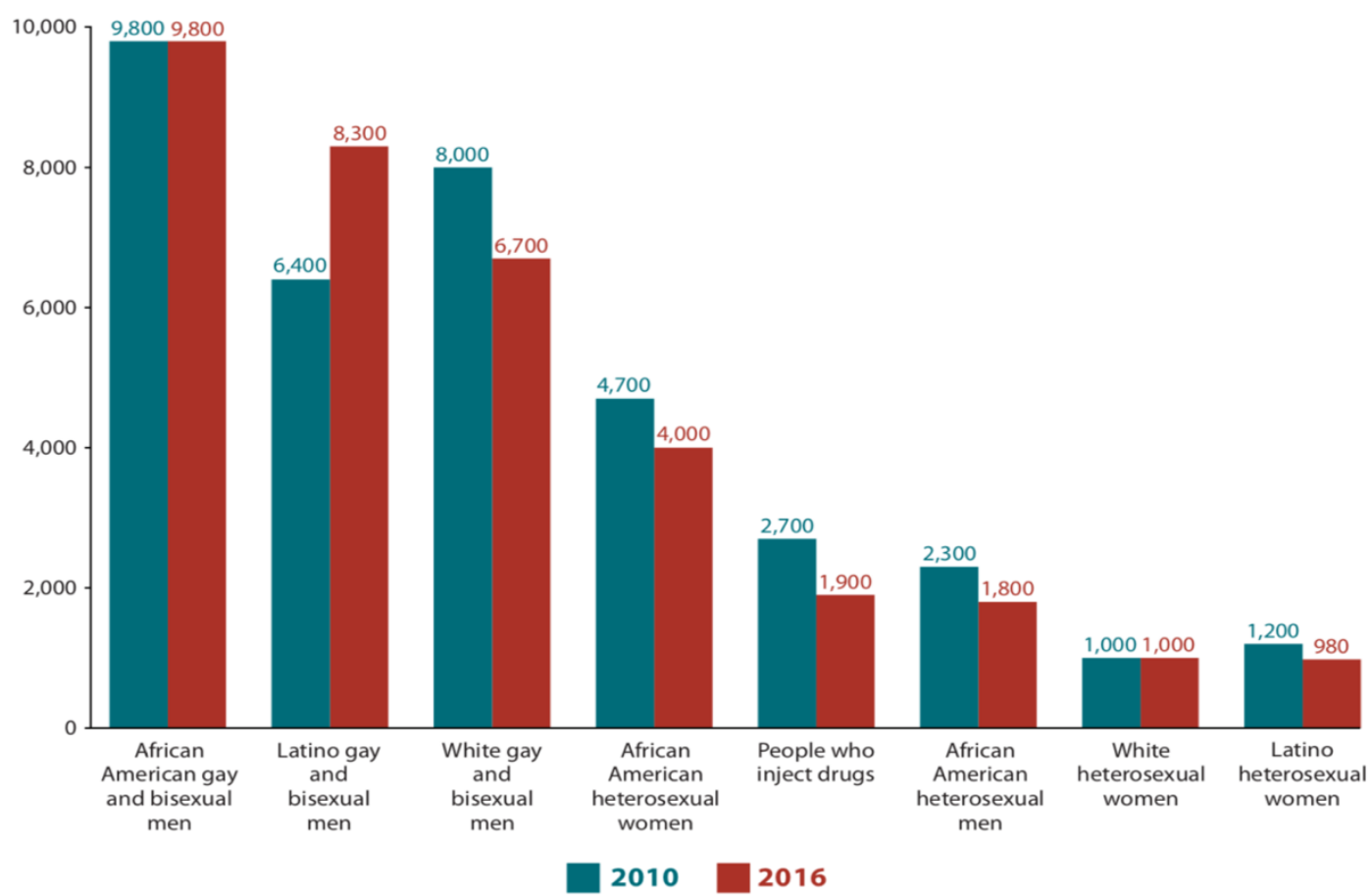

Source: CDC, HIV Incidence Estimated Annual Cases in the US., 2010-2016, Feb 2019.

Similar trends in HIV rates among African Americans have also been observed in the state of Kentucky. A report by Kentucky Cabinet for Health and Family Services Department for Public Health HIV/AIDS Branch (2017) indicates that Kentucky has seen an estimated 10,244 cases of diagnosed HIV since the beginning of the HIV/AIDS epidemic; and African Americans are disproportionately impacted. African Americans 
accounted for $37 \%$ of the newly diagnosed HIV cases in 2015 , despite only comprising 9\% of the total Kentucky population (Kentucky Cabinet for Health and Family Services Department for Public Health HIV/AIDS Branch, 2017).

Figure 2 HIV Cases increase among Latino and African American Gay and Bisexual Men Ages 25-34.

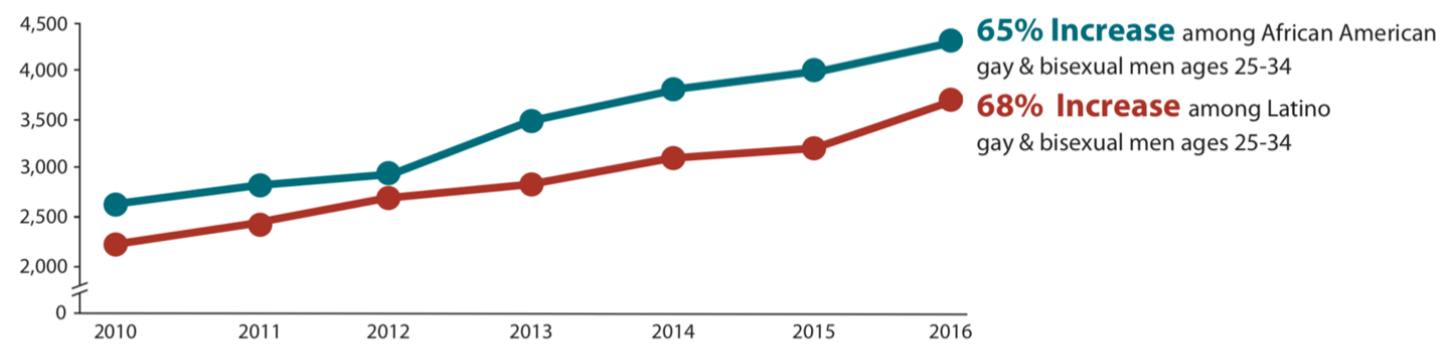

Source: CDC, HIV Incidence Estimated Annual Cases in the US., 2010-2016, Feb.2019.

Rates of newly diagnosed HIV cases were higher for African American men and women, compared to their White counterparts across a five-year trend (Figure 3). The report noted that African American men had 4.4 to 6.2 times higher rates than White males, while African American women had 9.6 to 12.8 times higher rates than White females over a five-year period.

Figure 3: Annual HIV Disease Diagnosis Rates by Race/Ethnicity, Sex, Year of Diagnosis, 2011-2015, in Kentucky.

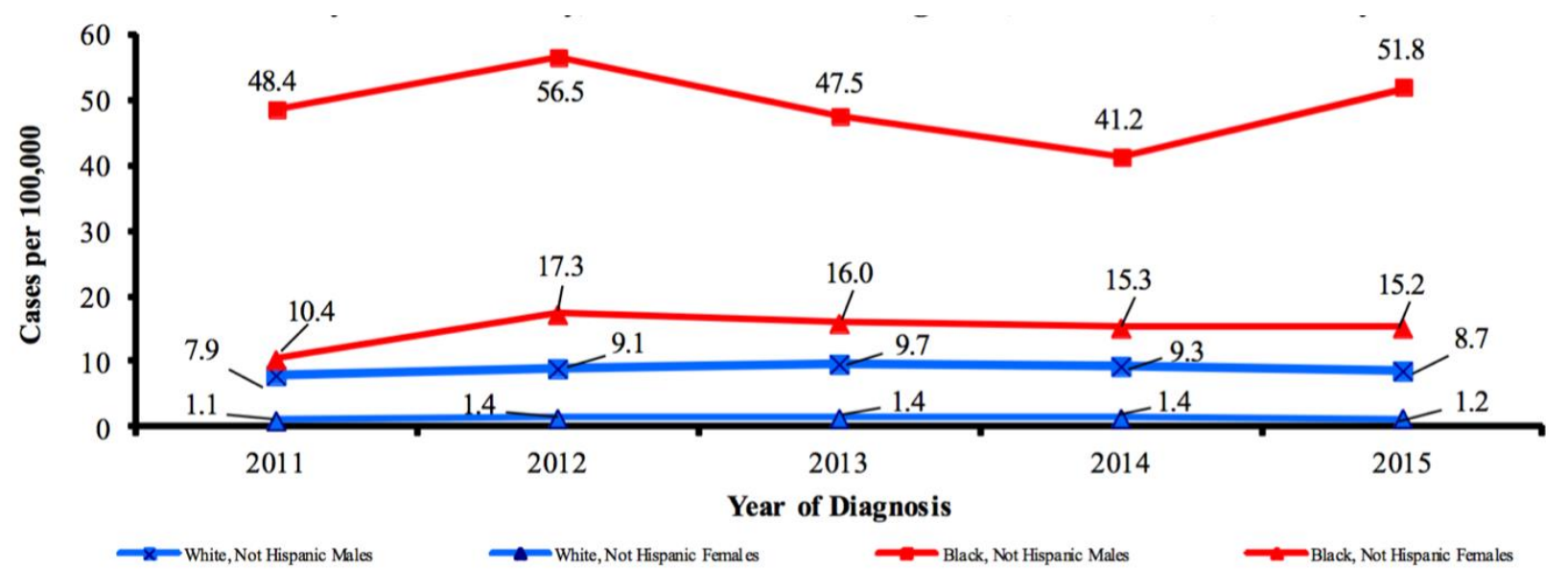

Source: Kentucky AIDS Surveillance Report, 2017. 


\section{African American Sub-Groups at High Risk for HIV}

While HIV rates are disproportionately high among African Americans, these high rates are not evenly distributed within this population. Sub-groups at high risk of HIV include (1) gender and sexual minorities, specifically men who have sex with men (MSM), transgender women (TGW); (2) persons who inject drugs (PWID); (3) high-risk heterosexual individuals such as (a) persons who exchange sex for money or other items, along with their partners; (b) serodiscordant couples, i.e. HIV-negative persons with HIV-positive partners; and (c) non-users of condoms whose partners have an unknown HIV status.

\section{Men who have sex with men (MSM)}

African American men who have sex with men (MSM) are some of the most heavily affected by HIV. The CDC estimates that more than half of all newly diagnosed HIV cases in the U.S. are among MSM, although this group only makes up $2 \%$ of the U.S. population (Centers for Disease Control and Prevention, 2018c). In Kentucky, MSM account for $56 \%$ of cumulative HIV cases reported among adults and adolescents (Kentucky Cabinet for Health and Family Services Department for Public Health HIV/AIDS Branch, 2017). MSM of color, especially African American MSM, experience disproportionately high HIV rates compared to their White counterparts. While White MSM saw a 10\% decline in HIV diagnoses between 2011-2015, African American MSM experienced a $4 \%$ increase in HIV diagnosis (Centers for Disease Control and Prevention, 2018i). This rise in HIV diagnosis was even more notable among African American gay and bisexual men ages 25-34 years old who experienced a $30 \%$ increase in diagnosis between 2011-2015 (Centers for Disease Control and Prevention, 2018a). As of 2016 African American MSM accounted for more than half (58\%) of HIV cases diagnosed 
among men (Centers for Disease Control and Prevention, 2018c) and the highest rates (38\%) of all new HIV diagnoses among MSM (Centers for Disease Control and Prevention, 2018a). Three quarters of all newly diagnosed HIV cases among African American MSM occur in young people between the ages of 13-34 years old (Centers for Disease Control and Prevention, 2018a).

\section{Transgender persons}

Transgender persons of color, especially transgender women who have sex with men, are also quite heavily affected by HIV. Transgender persons are individuals who identify with or expresses gender characteristics that are opposite to the sex they were assigned at birth (Centers for Disease Control and Prevention, 2016a). There are an estimated 1 million transgender adults in the U.S. (Centers for Disease Control and Prevention, 2018e). About $14 \%$ of transgender women reportedly have HIV, and $44 \%$ of African American transgender women have HIV (Centers for Disease Control and Prevention, 2018e). The CDC estimates that transgender women accounted for $84 \%$ of all HIV diagnoses among transgender persons between 2009 and 2014, and 51\% of those cases were among African American transgender women (Centers for Disease Control and Prevention, 2018e). Recent findings from the CDC HIV testing efforts among priority populations revealed that transgender women had the highest HIV diagnosis rates compared to other women and transgender men (Centers for Disease Control and Prevention, 2016a).

\section{Persons who inject drugs (PWIDs)}

Sharing syringes places PWIDs at an increased risk of acquiring HIV (Centers for Disease Control and Prevention, 2018d). In 2016, about 9\% of HIV diagnoses were 
attributed to injection drug use, and African Americans accounted for $31 \%$ of those cases (Centers for Disease Control and Prevention, 2018g). In Kentucky, injection drug use accounted for 10\% of cumulative HIV cases through 2017 (Kentucky Cabinet for Health and Family Services Department for Public Health HIV/AIDS Branch, 2017). These rates are expected to rise due to the national opioid crisis which has significantly impacted Kentucky (Centers for Disease Control and Prevention, 2018n). One study examined HIV and HIV-related behaviors in PWIDs across 20 U.S. cities in 2015. Using data from the CDC's National HIV Behavioral Surveillance (NHBS), they found that $27 \%$ of the HIVnegative PWIDs in the study reported sharing syringes, $67 \%$ engaged in condomless vaginal sex, and 22\% reported heterosexual condomless anal sex within the last 12 months prior to the study (Burnett, Broz, Spiller, Wejnert, \& Paz-Bailey, 2018). While sharing needles and engaging in condom-less vaginal and anal sex was more common among Whites than African American HIV-negative participants, they had the highest HIV prevalence (11\%) than Whites (6\%) among PWIDs (N=10,343) (Burnett et al., 2018). The high prevalence of HIV within the African American communities continues to place African American PWIDs at increased risk of HIV (Centers for Disease Control and Prevention, 2016c). Their vulnerability to HIV may be exacerbated by marginalization and discriminatory practices such as excessive policing, criminalizing laws, the war on drugs, inequitable sentencing, and mass incarceration that target African Americans (Kerr \& Jackson, 2016). 


\section{High-Risk Heterosexual Individuals \\ Commercial Sex Workers}

In addition to MSM and PWIDs, the HIV epidemic is also concentrated among other high-risk heterosexuals — commercial sex workers (CSWs) and their clients. The CDC contends that at least one of these groups (MSM, CSWs, and clients of CSWs) has a $>5 \%$ HIV prevalence rate compared to a $<1 \%$ prevalence rate in the general population (Centers for Disease Control and Prevention, 2017a). One meta-analysis of 14 studies on cisgender female sex workers from 1987-2013 reported a national pooled HIV prevalence of $17.3 \%$ with a wide variation in reported prevalence across individual studies (from 0.3\%-32\%) (Paz-Bailey, Noble, Salo, \& Tregear, 2016). Due to limited data on commercial sex workers, it is difficult to determine the precise HIV prevalence or incidence in this population. Evidence suggests that the odds of engaging in high-risk sex, i.e. sex without using condoms or with multiple partners of unknown HIV status, increases during transactional sex (Bobashev, Zule, Osilla, Kline, \& Wechsberg, 2009; Robinson \& Yeh, 2011). Individuals may also not be in a position to easily negotiate condom-use during sex (Centers for Disease Control and Prevention, 2016b). One study that examined correlates of transactional sex among drug users, MSM, and sex partners of these groups, the majority [76\%] of whom were African American, found associations between transactional sex and risky sexual behavior and other high-risk behaviors (Bobashev et al., 2009). Being African American, homeless, and having a history of incarceration and injection drug use was associated with higher odds of purchasing sex (Bobashev et al., 2009). Transactional sex is also common among low-income womenpredisposed to disadvantaged conditions and residing in areas prone to substance use. 
Many of these women are African American (Abad et al., 2015; Paz-Bailey et al., 2016;

Reilly et al., 2013; Sherman et al., 2018).

\section{Serodiscordant Heterosexual Couples}

Besides commercial sex workers and PWIDs, serodiscordant heterosexual couples and other heterosexual persons who do not use condoms or who have partners whose HIV status is unknown are at increased HIV risk. Nationally, heterosexual contact accounts for approximately $23 \%$ of HIV diagnoses (Centers for Disease Control and Prevention, 2017c). Approximately 87\% of HIV cases among young women are attributed to heterosexual contact, while only about $4 \%$ of HIV cases among young men are due to heterosexual contact (Centers for Disease Control and Prevention, 2018g). Crepaz, Dong, Chen, and Hall (2017) examined data from the national HIV surveillance system for diagnosed HIV cases between 2010-2015 and found that 6,000 cases per year in men and women were attributed to serodiscordant heterosexual contact (that is, sexual contact between a HIV-negative person with a HIV positive partner). In their study, heterosexual partnerships accounted for one in four HIV cases among men and five in ten HIV cases among women. HIV rates due to serodiscordant sexual activity were shown to be much higher among African Americans as well as persons residing in the southern part of the U.S.

\section{African American Heterosexual Women}

Heterosexual women account for $16 \%$ of all newly diagnosed HIV cases in the U.S., and African American women are disproportionally affected (Centers for Disease Control and Prevention, 2018f, 2018i). Although HIV rates among African American women have declined in recent years (Centers for Disease Control and Prevention, 
2018b), HIV rates for this sub-group remain alarmingly higher than rates for women of other races. In 2016 African American women accounted for 61\% (4,560) of HIV diagnosis among all women compared to $19 \%(1,450)$ for White women and $16 \%(1,168)$ for Hispanic/Latina women (Centers for Disease Control and Prevention, 2018f). One in five new HIV cases are among women, and African American women account for 59\% of those cases (Centers for Disease Control and Prevention, 2019a). The African American woman is 17 times more likely to be diagnosed with HIV compared to the White woman (Hess, Hu, Lansky, Mermin, \& Hall, 2017).

African American women are often predisposed to unfavorable life conditions and drastic social changes that may, in turn, directly or indirectly increase their risk of HIV. Researchers note that "the decline in marriage rates among black women, coupled with women's declining economic power and reduced partner options has shifted the balance of their gender power, making it difficult for many of them to negotiate condom use for self-protection" (Sharpe et al., 2012, p. 250). In addition to diminished gender power especially in negotiating safer sex practices -intimate partner violence, high prevalence of STIs, diminished male-female sex ratios due to excessive incarceration of African American males, and disrupted HIV care engagement of formally incarcerated partners have been shown to influence HIV risk among African American women (Adams et al., 2018; Braksmajer, Senn, \& McMahon, 2016; Sharpe et al., 2012). Overall, women of color often fare poorly in HIV outcomes because many "face significant discrimination as a result of race or ethnicity and sex, and they suffer disproportionately from poverty, low health literacy, and lack of access to high-quality HIV care" (Aziz \& Smith, 2011, p. S231) 


\section{Determinants of HIV Disparities among African Americans}

Health disparities do not exist in a vacuum (DiClemente, Salazar, \& Crosby, 2007). There are underlying systemic root causes of such avoidable differences observed among people based on their race, social status, class, gender, or sexual orientation etc. These forces transcend mere individual choices, behaviors, or genetics. To understand why health disparities exist, public health researchers consider several factors, including structural, social, societal, historical, and political circumstances, which are known to contribute to health outcomes of individuals, communities, and populations. For African Americans, the interaction between those factors such as where they live and work, as well as the resources available to them, may influence their health outcomes as well as their risks of exposure to diseases like HIV/AIDS. Such factors are referred to as social determinants of health (SDOH). These are conditions in which people are born, grow up, live, work and age, and the systems put in place to deal with illness, which are then influenced by policies, economic, social, and political forces (World Health Organization, 2016). Individual health risks and outcomes are mediated by SDOH. These may fuel the disparities in health outcomes seen across different populations as is the case for HIV/AIDS among African Americans. Some determinants of HIV among African Americans include, but are not limited to, racial and ethnic segregation (Kahana et al., 2016), lack of trust in the health care system, conspiracy beliefs (Bogart \& Thorburn, 2005), socio-economic factors (housing and means of transportation), high incarceration rates (which influence sexual networks), HIV-related stigma (Adimora \& Schoenbach, 2005; Kerr \& Jackson, 2016; Kerr, Valois, DiClemente, et al., 2015; Reif et al., 2014), and homophobia and homonegativity (Adimora \& Auerbach, 2010). 


\section{Examining Various HIV Prevention Approaches}

Until recently, intervention approaches employed in the battle against HIV/AIDS have been individual-level and behavior-focused. Individual-level interventions target specific behaviors or individual factors without necessarily taking into consideration other factors (such as the environment or culture) which may influence individual behaviors. Since HIV is mostly transmitted through sexual contact, many studies focus on abstinence, condom use, delaying sexual initiation, reducing number of sexual partners, and acquiring sexual negotiation skills (Cardoza, Documét, Fryer, Gold, \& Butler, 2012; DiClemente et al., 2007; Johnson, Scott-Sheldon, Huedo-Medina, \& Carey, 2011; Kirby, Laris, \& Rolleri, 2007). However, evidence suggests that sexual-risk behaviors alone may be limited in explaining the disparities in HIV rates among African Americans (Gant, Gant, Song, Willis, \& Johnson, 2014). Researchers argue that structural factors, rather than mere individual behaviors, fuel the HIV epidemic (Dean \& Fenton, 2010; Kahana et al., 2016; Wohlfeiler \& Ellen, 2007). While behavior-focused interventions have been shown to be effective in reducing HIV risk, this approach is limited and its effects often short-lived (DiClemente et al., 2007). Behavioral interventions (which often rely on individual-level models) alone are limited in preventing HIV, since factors beyond the control of individuals may shape their risks of exposure to diseases like HIV.

Individual behaviors do not exist in isolation but are, rather, intertwined or influenced by other factors such as interpersonal, social, economic and cultural contexts which may restrain or promote such behaviors (DiClemente et al., 2007). Thus, interventions employing a multi-level approach tend to hold better promise in effecting behavior change in the long term (DiClemente et al., 2007). Multi-level approaches target 
social and economic factors, racial disparities, environmental, and political factors that may influence HIV risk (Ellen et al., 2015; Gant et al., 2014; Kahana et al., 2016; Wohlfeiler \& Ellen, 2007). These approaches often employ theoretical models like the social ecological model (SEM) (Baral, Logie, Grosso, Wirtz, \& Beyrer, 2013;

DiClemente et al., 2007). They may employ various levels of the SEM or combination of levels influencing HIV including the individual-level (biological and behavioral factors influencing HIV risk), group-level, interpersonal-level (social support/networks), community-level (organizational/institutional interactions), and policy-level (laws at the local, state, and national level) (Baral et al., 2013). Evidence suggests that comprehensive, multi-level/multifaceted approaches, such as those which combine several levels of the social ecological model, are more efficacious in preventing HIV (Charania et al., 2011; Jackson, Geddes, Haw, \& Frank, 2012; Prado, Lightfoot, \& Brown, 2013).

Moreover, no intervention implemented at any single level of the SEM can considerably account for all the factors, including the structural barriers and enablers within an individual's environment that may be critically important in influencing individual behaviors. This underscores the importance of much broader, more comprehensive, multi-level approaches to behavior change in addressing HIV risk (Baral et al., 2013; DiClemente et al., 2007; Wohlfeiler \& Ellen, 2007). Employing a multifaceted approach that entails decreasing barriers and improving access to high-impact biomedical interventions, like HIV Pre-Exposure Prophylaxis (PrEP), is expected to positively improve HIV prospects for high-risk groups. 


\section{Introduction to Pre-Exposure Prophylaxis (PrEP)}

Biomedical interventions, which typically involve the use of antiretroviral therapies (ART), have shown promise in the fight against HIV. Early initiation of ART has been shown to reduce the risk of sexual HIV transmission at a high efficacy rate (96\%) among partners in serodiscordant relationships who initiated early ART (Cohen et al., 2011; Cohen, Smith, et al., 2013). Studies have demonstrated that proper uptake of ART greatly reduces viral load (blood levels of virus) to undetectable levels (that is, amount of virus in plasma falls below 50 copies per milliliter of blood), thus making the chances of transmitting the virus slim to none (Attia, Egger, Müller, Zwahlen, \& Low, 2009; Crepaz et al., 2017; Gardner, McLees, Steiner, del Rio, \& Burman, 2011). This has implications for HIV prevention among various high-risk groups. Consequently, ART has been recommended and utilized in the form of post-exposure prophylaxis (PEP) and preexposure prophylaxis (PrEP) for preventing HIV in high-risk individuals (Abbas, 2011; Baeten \& McCormack, 2016; Baeten, Haberer, Liu, \& Sista, 2013; Cohen, Liu, Bernstein, \& Philip, 2013; Grant \& Smith, 2015; Smith et al., 2005).

HIV PrEP, in particular, has shown promise in significantly impacting the HIV epidemic and decreasing disparities (Centers for Disease Control and Prevention, 2017f). PrEP is commonly marketed as Truvada. In 2012, the FDA approved Truvada - a combination of emtricitabine (FTC) and tenofovir (TDF) — as a PrEP agent for HIV prevention (U.S. Food and Drug Administration, 2012). However, other PrEP medications may only contain either TDF or FCT (Lehman et al., 2015). Truvada, when taken daily, has been proven to decrease the number of new HIV cases in high-risk groups (Dolling et al., 2016; Grant et al., 2010). Descovy, another PrEP medication, 
received FDA approval in 2019, however, indications for use did not include cisgender women, since at the time of the approval, the effectiveness of Descovy had not been evaluated in persons at risk for contracting HIV through receptive vaginal sex (U.S. Food and Drug Administration, 2019).

Numerous clinical trials and demonstration projects (Baeten et al., 2012; Choopanya et al., 2013; Grant et al., 2010; McCormack, Dunn, Desai, Dolling, Gafos, Gilson, Sullivan, Clarke, Reeves, Schembri, et al., 2016) conducted domestically and internationally have established the safety and effectiveness of these drug regimens in preventing HIV cases through primary prevention (U.S. Food and Drug Administration, 2012). PrEP is said to be "well tolerated and highly effective for HIV prevention - for men and women, cisgendered and trans-gendered [sic], with different HIV exposures, across the globe" (Baeten \& McCormack, 2016, p. 1). It has been shown to be safe and effective in men and transgender women who have sex with men, with a reported $44 \%-$ 86\% reduction in HIV incidence (Dolling et al., 2016; Grant et al., 2010). Some early women-only trials in South Africa indicated lack of effectiveness of PrEP among women, which was cause for concern (Celum \& Baeten, 2012). However, a series of metaanalysis of current evidence on randomized controlled trials, which included women, indicated effectiveness of PrEP with high levels (75\%) of adherence (Hanscom et al., 2016). Effectiveness has also been shown for heterosexual men and women (Baeten et al., 2012; Elion \& Coleman, 2016). PrEP, however, does not protect $100 \%$ against HIV acquisition. Also, resistance to antiretroviral medications is possible in persons who become HIV infected while taking PrEP; this effect, however, appears to be minimal (Lehman et al., 2015). Kaiser Permanente in San Francisco (KPSF) reported successful 
implementation of a specialized PrEP program among its large integrated health care system, resulting in no new HIV cases among PrEP users since July 2012 (Volk et al., 2015).

Despite the efficacy of PrEP, several challenges from the perspectives of providers and eligible or potential PrEP users influence its uptake (these will be explored in greater detail the next chapter). Challenges to PrEP uptake range from awareness to beliefs and perceptions of PrEP that, in turn, affect its availability, accessibility, and acceptability by populations who need it the most. Examples include provider knowledge, awareness, attitudes, concerns, and prescribing practices (Hakre et al., 2016; Karris, Beekmann, Mehta, Anderson, \& Polgreen, 2014; Krakower, Ware, Mitty, Maloney, \& Mayer, 2014; Petroll et al., 2017). Other examples from eligible or potential PrEP-user perspectives include lack of willingness to use PrEP, mistrust of the health care system, stigma, and negative misconceptions (Eaton, Driffin, Bauermeister, Smith, \& Conway-Washington, 2015; Eaton, Driffin, Kegler, et al., 2015; Mutchler et al., 2015)

\section{CDC Guidelines for Administering PrEP}

In 2014, the CDC released comprehensive PrEP-administering guidelines for providers to facilitate PrEP delivery to HIV-negative individuals at substantial risk of HIV (Centers for Disease Control and Prevention, 2014b). The individuals considered to be at substantial risk of acquiring HIV include serodiscordant couples (those in an ongoing relationship with an HIV-positive partner only in situations where the HIV positive partner is neither taking antiretroviral medications nor has a suppressed viral load); heterosexual men and women who do not regularly use condoms during sex with partners of unknown HIV status (who are at substantial risk such as injection drug users); 
gay or bisexual men who have had anal sex without a condom in the past 6 months or have been diagnosed with an STD; and injection drug users in the past 6 months who share injection equipment or in drug treatment for injection use in the last 6 months. In 2017, the CDC revised its guidelines to improve clarity in clinical care and to update developing evidence base for PrEP efficacy and uptake (Centers for Disease Control and Prevention, 20181). For example, in the revised clinical guidelines, the CDC removed the requirement for injection drug users to be in treatment for injection use in the last 6 months preceding PrEP engagement as this was confusing to clinicians (Centers for Disease Control and Prevention, 20181).

One shortfall of this initiative is that the CDC failed to specifically list African American women in its PrEP clinical guidelines as one of the groups at substantial risk of HIV. Yet, according to the CDC, African American women are highly vulnerable to HIV(Centers for Disease Control and Prevention, 2019a). One in five new HIV cases are among women, African American women account for 59\% of those cases (Centers for Disease Control and Prevention, 2019a). Also, gender disparity gaps in PrEP-use among persons indicated for PrEP in the U.S. have been reported. For instance, only 4.7\% (of 78,360) PrEP perceptions filled in 2016 were filled by women (Huang, Zhu, Smith, Harris, \& Hoover, 2018). Despite this, there is a dearth of studies focusing on examining PrEP uptake among U.S. women (Auerbach, Kinsky, Brown, \& Charles, 2015).

\section{Problem Statement}

The use of PrEP is a promising approach for preventing HIV in various at-risk populations (Abbas, 2011; Baeten \& McCormack, 2016; Baeten et al., 2013; Grant \& Smith, 2015). PrEP has been shown to be effective, but various concerns threaten to stifle 
its uptake. Despite the promise of this intervention in slowing down HIV rates among various high-risk groups, uptake remains a challenge (Adams \& Balderson, 2016; Petroll, Staden, \& Westergaard, 2016). This slow uptake of PrEP is especially common among African American groups (Eaton, Driffin, Bauermeister, et al., 2015). Thus, additional research is warranted to develop a deeper understanding of the various factors influencing low PrEP uptake among African American high-risk groups.

\section{Goal}

The overarching goal of this dissertation is to examine and address factors related to low PrEP engagement and outreach that impact PrEP uptake among African American youth in Louisville, KY at high risk for HIV.

\section{Purpose of The Study}

The purpose of this dissertation study was three fold: (1) to qualitatively explore and understand the barriers and facilitators to PrEP uptake among various African American youth (18-29 years old) around Louisville, Kentucky in priority groups (persons demonstrating heightened HIV risk), (2) to examine effective strategies for scaling up PrEP engagement and outreach among African American priority groups, and (3) to develop recommendations for local AIDS service organizations (ASOs) to improve PrEP outreach/delivery efforts among African American priority groups.

\section{Research Questions}

The following research questions were posed for this dissertation study:

R1. What are the multi-level barriers and facilitators to PrEP engagement, from the perspectives of African American youth groups in Louisville, KY who are at high risk for HIV? 
R2. What are the strategies, nationally, for PrEP outreach and delivery among various groups at high risk for HIV, particularly African Americans, from the perspectives of key informants in ASOs across the country?

R3. How should ASOs in Louisville, KY approach PrEP outreach/delivery with African American groups at high risk to improve PrEP engagement, based on evidence from research questions 1 (local context) and 2 (national context)?

\section{Study Aims}

The dissertation study had three specific aims:

A1. To explore and understand barriers and facilitators to, and engagement with, PrEP uptake among priority groups.

A2. To develop a context-specific framework grounded in experiences of ASOs of how they have successfully implemented PrEP outreach among African American priority high-risk groups.

A3. To identify and describe effective strategies in the form of recommendations for ASOs in Louisville, KY to improve service delivery and outreach to African American priority high-risk groups for PrEP engagement.

\section{Rationale for study population selection}

There were three target populations in this dissertation:

1. African American youth (18-29 years old) in priority high-risk groups

2. ASOs in Louisville, Kentucky

3. ASOs outside of Kentucky (national sample) 


\section{African American High-Risk Youth Groups}

For the first target population, this dissertation focused on African American youth (18-29 years old) who are at high risk for HIV and who fit any of these high-risk categories: (1) gender and sexual minorities and (2) high-risk heterosexual individuals (male and female). A third desired category, persons who inject drugs (PWID), was not possible because no one recruited to participate in the AFYA study (from which data for this portion of the dissertation was derived) self-identified as PWID. The selection of risk categories was informed by the CDC recommendations for engaging persons at substantial risk of HIV (Centers for Disease Control and Prevention, 2014b). The age selection was informed by U.S. and Kentucky data, which depict high HIV incidence among youth, particularly in the age group of 20-29 year-olds (Figures 4 \& 5) (Centers for Disease Control and Prevention, 2019e; Kentucky Cabinet for Health and Family Services Department for Public Health HIV/AIDS Branch, 2017).

Figure 4 New HIV Diagnosis, 2017.

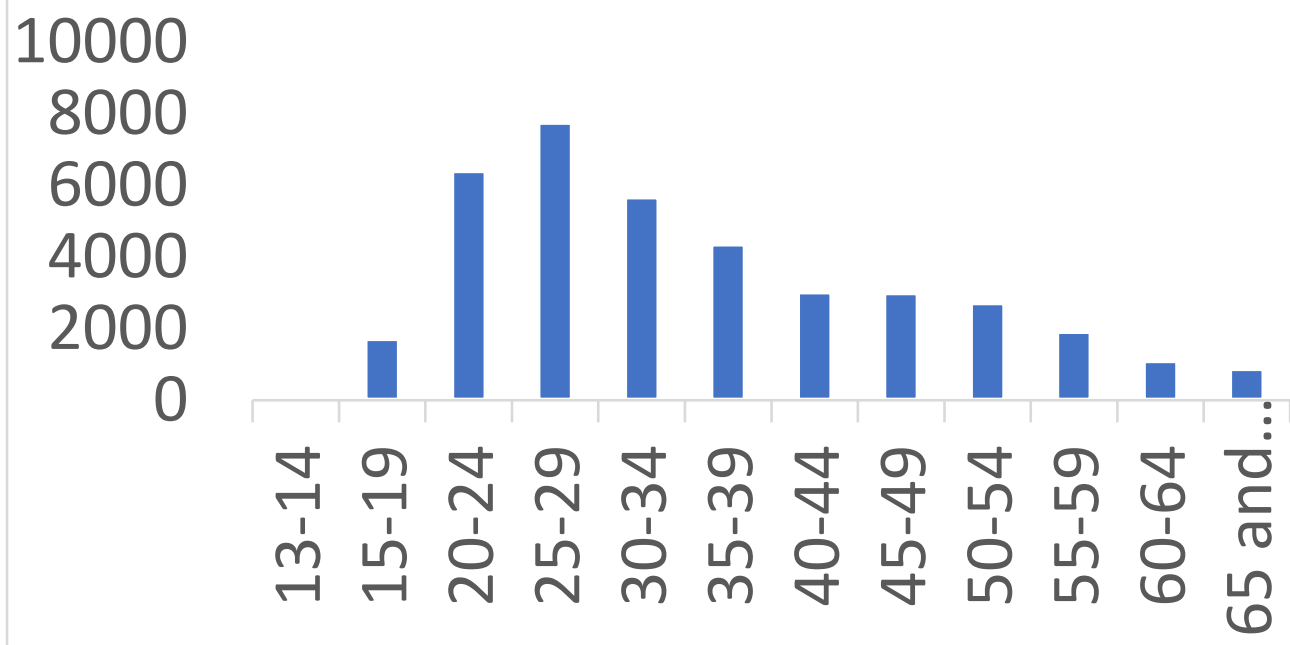

Source: CDC Statistics Overview, 2019. 
While HIV affects all ages, young people (particularly youth between the ages of 20-29 years old) are especially at heightened HIV risk. The CDC estimates that more than half of young people living with HIV are unaware of their status, and for those who have been diagnosed, less than half are getting the care that they need to keep their viral loads suppressed to avoid transmitting the virus to others (Zanoni \& Mayer, 2014). These alarming HIV rates among young people represent the highest rates of undiagnosed HIV as well as the lowest rates of viral load suppression seen in any other age group (Centers for Disease Control and Prevention, 2018g). In 2016, approximately 1 in 5 (21\%) new HIV cases diagnosed that year were among youth 13-24 years old, and 20-24-year-olds accounted for an estimated $80 \%$ of all new cases among youth (Centers for Disease Control and Prevention, 2018g). Additionally, while rates of HIV diagnoses remained stable in the U.S. between 2012-2016, they increased for young persons, ages 25-29 years old (Centers for Disease Control and Prevention, 2017e). In 2017, 25-29-year-olds had the highest rate $(32.9 \%)$ and 20-24-year-olds had the second highest rate $(28.7 \%)$ of all newly diagnosed HIV cases in the U.S. compared to the national average rate of $11.8 \%$ (Centers for Disease Control and Prevention, 2017e). Young African Americans had the highest rates, compared to their White and Hispanic counterparts (Centers for Disease Control and Prevention, 2018g). The majority (93\%) of new HIV cases diagnosed among youth in 2017 were attributed to male-to-male contact, and higher among African American youth (Centers for Disease Control and Prevention, 2018g). African American male-to-male contact accounted for $51 \%$ (of 6,800) compared to $25 \%$ among Hispanics and 18\% among Whites (Figure 5). 
Figure 5 New Diagnosis Among Young Gay and Bisexual Men by Race/Ethnicity in the US and Dependent Areas, 2017.

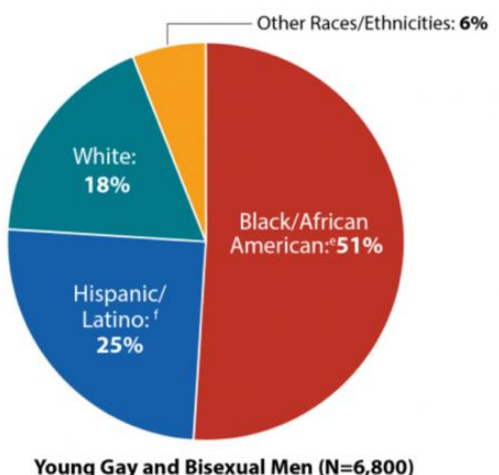

Source: CDC HIV surveillance Report, 2017.

Kentucky annual HIV trends also show that 20-29-year-olds have the highest rates of newly diagnosed HIV cases compared to other age groups (Figure 6). Locally, Jefferson County/Louisville Metro (henceforth referred to as Louisville metro) in Kentucky has the highest rates of HIV diagnosis in Kentucky (Kentucky Cabinet for Health and Family Services Department for Public Health HIV/AIDS Branch, 2016). These rates are higher among young people, many of whom are African American.

Figure 6 HIV Disease Diagnosis Rate by Age and Year of Diagnosis, 2011-2015, Kentucky.

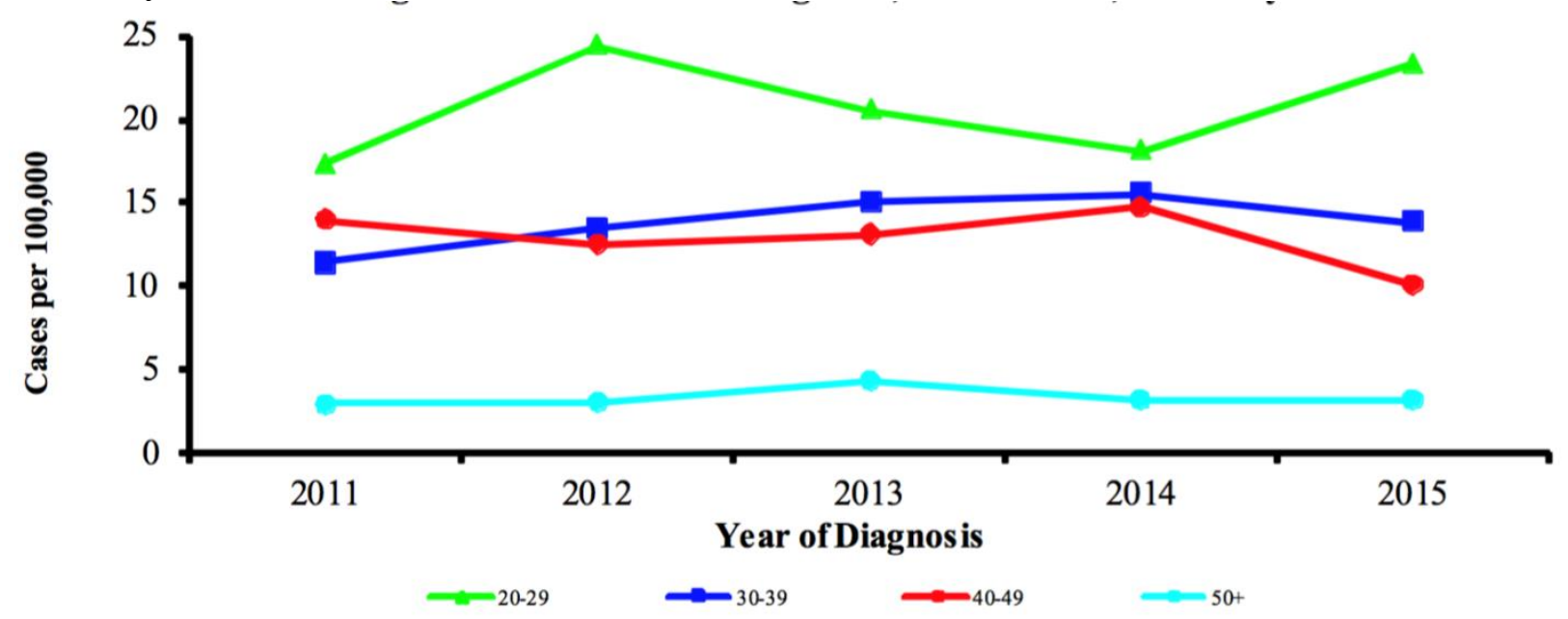

Source: Kentucky AIDS Surveillance Report, 2017. 


\section{AIDS Service Organizations (ASOs)}

The choice to involve ASOs in this study stems from the vital role they play in HIV prevention. These service agencies may have existing relationships with the communities within which they serve and hold the potential to access populations considered to be at heightened risk for HIV (Owczarzak \& Dickson-Gomez, 2011). ASOs are well positioned because of their current work in HIV prevention, such as HIV/STD testing services. In one study, ASOs as well as local clinics and community health centers providing HIV testing and other STD services were instrumental to PrEP delivery, as they provided referrals or showed potential clients how and where to access PrEP services (Elopre, Kudroff, Westfall, Overton, \& Mugavero, 2017). However, research also indicates that few ASOs are adequately engaging with African Americans in priority groups who could benefit from PrEP (Elopre et al., 2017; Smith et al., 2016). This underscores the need to assess and address factors impacting the ability of ASOs to adequately engage African Americans with PrEP.

Thus, this dissertation assessed current PrEP-implementing practices among two ASO samples: (a) a national sample of ASOs who have demonstrated success with PrEPengagement efforts among African Americans in priority groups and (b) a sample of Louisville ASOs who have the potential to engage African American priority groups with PrEP for HIV prevention, but may need to scale up their efforts (results of this portion of the study are described elsewhere and currently under review) (Ayangeakaa et al., 2020). The dissertation further aimed to utilize findings from the national ASO sample as well as the study with the priority populations to inform the efforts of Louisville ASOs in scaling up PrEP outreach and engagement among this population with high HIV vulnerability. 
Assessment of the Louisville ASO sample mainly served as background to developing recommendations that can be tailored to local ASO's needs.

\section{Conclusion}

African Americans experience disproportionate rates of HIV, and some subgroups within this population who practice high-risk behaviors are at greater risk of becoming infected. Despite various interventions, which have been mostly behavioral in nature until recently, disparity gaps in HIV rates among African Americans have persisted. HIV PrEP holds promise to slow down HIV rates and decrease disparities, but uptake is low among African Americans. Therefore, it is imperative to explore multilevel factors influencing PrEP uptake among African Americans and to determine effective strategies for engaging with African Americans youth in various high-risk groups. Assessing individual factors pertaining to PrEP use and access among various African American youth risk groups as well as structural factors influencing PrEP engagement among them is expected to help identify effective strategies for improving HIV prevention and reducing disparities. Also, since most studies focus mainly on MSM and bisexual males without adequately exploring other high-risk groups, this study attempted to fill that gap in the literature by including youth in other high-risk categories (in addition to MSM and bisexual) indicated for PrEP and recommended by the CDC.

\section{Definition of Terms}

African American: a person having origins in any of the Black racial groups of Africa (Census.gov, 2011). 
AIDS Service Organizations (ASOs): this refers to a community-based organization (CBO) providing services related to prevention and or treatment of HIV/AIDS (AIDSinfo.nih.gov, 2019). For the purposes of this study, an AIDS service organization (ASO) includes CBOs and health departments and university hospitals who are providing PrEP delivery services, with emphasis on only ASOs conducting outreach within the community.

Biomedical Prevention: refers to use of medications to prevent the spread of HIV cases. Cisgender: refers to "a person whose gender identity corresponds with the sex the person had or was identified as having at birth" (“Cisgender", 2019).

Community-Based Organization (CBO): refers to any organizations based within the community that provides various support services or health care services including, but not limited to, HIV services.

Heterosexual: characterized by a tendency to direct sexual desire toward the opposite sex ("Heterosexual", 2019).

High-Risk: refers to individuals at "substantial risk" for HIV as defined by the CDC: any one possessing characteristics fitting any one of these groups: serodiscordant couples (those in an ongoing relationship with an HIV-positive partner), any heterosexual man or woman who does not regularly use condoms, and gay or bisexual men who have had anal sex without a condom in the past 6 months or have been diagnosed with an STD and injection drug users in the past 6 months who share injection equipment (Centers for Disease Control and Prevention, 2014b). 
High-Risk Youth: for the purposes of this dissertation, refers to any African American young person between the ages of 18-29 years old who fits the definition of high-risk groups above.

PrEP Service Delivery: refers to any services relating to PrEP education, referral, follow-up, adherence support, and onsite PrEP prescribing services.

PrEP Outreach: refers to any activity that serves the purpose of locating, recruiting, and engaging clients and target audiences for the purpose of providing PrEP services.

PrEP Engagement: refers to the acceptability of PrEP as an option for HIV prevention as well as willingness to become informed and educated about PrEP such as through PrEP promotion efforts and outreach efforts of interventionists like ASOs.

Serodiscordant Couple: refers to relationships, in which only one partner is HIVpositive, while the other partner is HIV-negative (USAID.gov).

Sex Workers: Anyone who engages in transactional sex; that is, exchanging sex for money or other items (Bobashev et al., 2009).

Social Determinants of Health: These are conditions in which people are born, grow up, live, work, and age, and the systems put in place to deal with illness, which are then influenced by policies, economic, social, and political forces (World Health Organization, 2016).

Transgender: refers to "a person whose gender identity differs from the sex the person had or was identified as having at birth" (“Transgender", 2019).

Treatment as Prevention: the use of antiretroviral medications by HIV-positive individuals to reduce HIV transmission (McCormack, Gafos, Desai, \& Cohen, 2014). 


\section{CHAPTER II}

\section{LITERATURE REVIEW}

\section{Introduction}

The previous chapter provided a brief background about the HIV epidemic in the U.S. and the importance of biomedical interventions like PrEP for preventing HIV. HIV PrEP is a promising approach to HIV prevention with the potential to reduce infectivity rates and narrow disparity gaps, but uptake remains low among African American highrisk groups. The chapter also pointed to the potential role AIDSs service organizations (ASOs) could play in improving PrEP uptake among African American groups within their local communities.

In this chapter, I explore literature on the underlying factors influencing high rates of HIV and low PrEP uptake among African Americans. To achieve this aim, this chapter provides a review of national and state (Kentucky) trends in HIV rates with a focus on African Americans, and a review of research findings regarding HIV and PrEP uptake disparities, challenges, and intervention strategies previously explored within this population.

Structurally, the chapter comprises seven main sections with overarching themes, accompanied by several relevant sub-sections under each main section. The first section gives a focused overview of HIV trends across the U.S. and in the state of Kentucky, and a rationale for targeting African American high-risk groups. The second section describes 
the impact of social determinants of health and other factors on HIV disparities among African American groups. The third section provides a brief overview and background to PrEP. The fourth discusses PrEP for HIV prevention, highlighting factors surrounding PrEP uptake among African Americans. The fifth section examines the role of community-based organizations such as AIDS service organizations (ASOs) in increasing PrEP uptake within the community, especially among high-risk African American groups. The sixth section addresses gaps in the literature and provides a rationale for the study. The seventh section discusses the theoretical frameworks guiding the dissertation. The chapter concludes with a summary of each section.

\section{Overview of HIV Trends Across the U.S.}

\section{National Trends}

In the U.S., approximately 1.1 million people are living with HIV and approximately 1 in $7(14.3 \%)$ of them are unaware of their HIV positive status (Centers for Disease Control and Prevention, 2018j; HIV.gov, 2017; Laufer, O'Connell, Feldman, \& Zucker, 2015; Reif, Safley, McAllaster, Wilson, \& Whetten, 2017). In the last decade, an average of 50,000 newly diagnosed HIV cases were observed every year in the U.S. (Centers for Disease Control and Prevention, 2013), but the HIV incidence rate has been declining in recent years. Between 2010 to 2015 , the rates of newly diagnosed HIV cases declined approximately $8 \%$ (from 41,800 to about 38,500 , respectively) due to advances in prevention (Centers for Disease Control and Prevention, 2018i).

While HIV/AIDS does not discriminate based on age, gender, race/ethnicity, religion, and sexual orientation, disparities do exist in the distribution of morbidity and mortality. These disparities cut across racial/ethnic lines as well as gender and 
geographical locations (Centers for Disease Control and Prevention, 2018h, 2018i; Reif et al., 2014). Geographically, HIV is not evenly distributed, and rates are more heavily concentrated in the U.S. South. In 2016, more than half of the newly diagnosed HIV and AIDS cases were in the southern states; they had the highest rates of HIV diagnosis (16.8 per 100,000 persons), compared to other regions in the country (Northeastern, West, and Midwestern regions each had 11.2, 10.2, and 7.5, respectively) (Centers for Disease Control and Prevention, 2018h, 2018i).

The disproportionate distribution of HIV rates also exists along racial and ethnic lines. For instance, minority populations such as African Americans and Hispanics often bear the highest burden of HIV and AIDS compared to their White counterparts. Among the different races/ethnicities, African Americans are disproportionately affected (Centers for Disease Control and Prevention, 2018h). By the end of 2013, African Americans had the highest death rate $(15.3$ per 100,000) from HIV cases ever diagnosed as stage 3 (AIDS), compared to Hispanics (4.1) and Whites (2.0). By 2014, African Americans also had the highest estimated rates of cases classified as AIDS (25.4 per 100,00 population) compared to Hispanics/Latinos (7.7) and Whites (2.7) (Centers for Disease Control and Prevention, 2014a). Additionally, according to CDC estimates, the cumulative number of persons who have ever been diagnosed with AIDS in the U.S was approximately $1,232,346$ by the end of 2016, and African Americans accounted for $41 \%(508,711)$ of that number (Centers for Disease Control and Prevention, 2017d).

\section{Local (State of Kentucky) Trends}

Disparities in HIV rates do not only exist at the national level. According to a report by Kentucky Cabinet for Health and Family Services Department for Public Health 
HIV/AIDS Branch (2017), disproportional HIV rates are also observed across age groups, with young people (20-29 years old) experiencing the highest rates of diagnosis. Of all the newly diagnosed HIV cases across the state of Kentucky in 2015, young people ages 20-29 had the highest rates in general (Figure 6). The report also noted that, "the rates among [African Americans] in all age groups were at least four times higher than the rates among their White counterparts of the same age group" (Kentucky Cabinet for Health and Family Services Department for Public Health HIV/AIDS Branch, 2017, p. 23).

Figure 6 HIV Disease Diagnosis Rate by Age and Year of Diagnosis, 2011-2015.

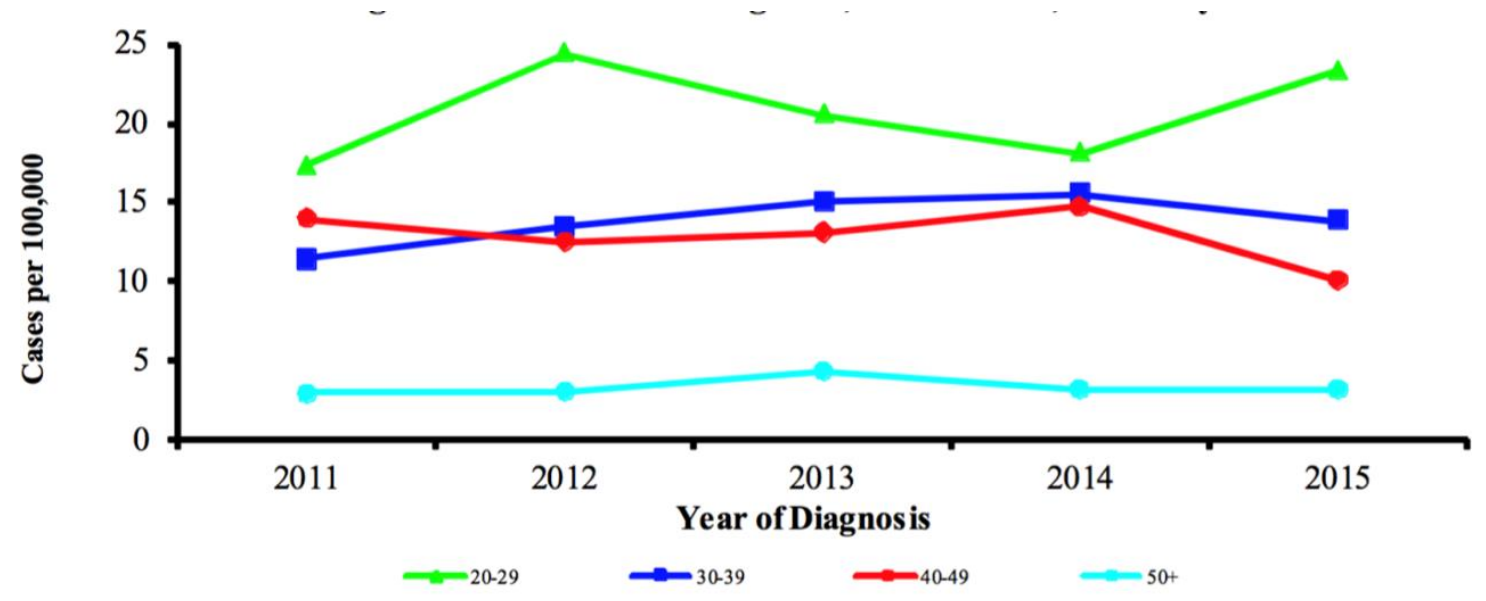

Source: Kentucky AIDS Surveillance Report, 2017.

HIV rates are expected to rise in the state of Kentucky, owing to the opioid crisis. Kentucky is home to 54 of 200 vulnerable counties that the CDC estimates are at risk of an HIV outbreak due to injection drug use. Many of these are in the Appalachian mountains (Centers for Disease Control and Prevention, 2018n; Ungar, 2017). Kentucky is one of the top 10 states in the nation to be affected by the opioid crisis (National Institute on Drug Abuse, 2018). In 2016, Kentucky experienced deaths from opioid overdose at a rate (23.6 deaths per 100,000 persons) almost double the national average 
rate (13.3 per 100,000 persons), and many of these overdose deaths were related to heroin (National Institute on Drug Abuse, 2018). According to the CDC, increased incidence of viral cases is a deadly consequence of the opioid crisis, given that the use of contaminated injection drug equipment is considered a primary route of transmission for blood-borne cases like HIV and hepatitis C (Centers for Disease Control and Prevention, 2018k). Kentucky is said to be poised for the next HIV outbreak, similar to the one which hit Austin, IN, “a tiny city with scarce medical resources where HIV sickened more than 200 people out of a population of 4,200" in 2015 (Ungar, 2017). An HIV outbreak could be complicated by the recent history of increased opioid abuse, along with injection drug use, complicated by poverty, low income, and unemployment observed in many rural counties around the state (Ungar, 2017).

Locally, out of the 15 various area development districts (ADD) in Kentucky, the KIPDA/North Central Regional Area, where Jefferson County is located (which includes the city of Louisville), had the highest rates (48\% or 4,930) of cumulative HIV cases diagnosed through 2017. Jefferson County, in turn, had the highest number of cases $(4,524)$ among the seven counties in this particular ADD (Kentucky Cabinet for Health and Family Services Department for Public Health HIV/AIDS Branch, 2017).

\section{Factors Driving HIV Disparities Among African Americans}

Health disparities — differences in health outcomes among various groups based on race, gender, social economic status, or other characteristics — are well documented. According to Healthy People 2020, health disparities are defined as types of health differences closely linked with social, economic, and/or environmental disadvantage (Healthy People 2020, 2014). These "adversely affect groups of people who have 
systematically experienced greater obstacles to health based on their racial or ethnic group; religion; socioeconomic status; gender; age; mental health; cognitive, sensory, or physical disability; sexual orientation or gender identity; geographic location; or other characteristics historically linked to discrimination or exclusion" (Healthy People 2020, 2014).

\section{Social Determinants of HIV Vulnerability}

A deeper look at determinants of HIV among African Americans may shed some light on why the disparities exist and how they can be adequately addressed. For many groups like African Americans, the interaction between factors like where they live and work and the resources available to them may influence their health outcomes as well as their risks of exposure to diseases. These factors are referred to as social determinants of health (SDOH). These are conditions in which people are born, grow up, live, work and age, and the systems put in place to deal with illness, which are then influenced by policies, economic, social, and political forces (World Health Organization, 2016). Individual health risks and outcomes are mediated by SDOH. These do fuel the disparities in health outcomes seen across different populations as is the case for HIV/AIDS among African Americans. Evidence suggests that structural factors (which constitute social determinants of health) such as living in disadvantaged settings, poverty, unemployment, racial and ethnic segregation, to name a few, may increase vulnerability to HIV (Kahana et al., 2016).

Marmot et al. (2010) in the report Fair Society, Healthy Lives argue that social and economic differences in health status (that drive health inequalities) cannot simply be attributed to genetics, unhealthy behaviors, or limited access to medical care; these are 
rather a reflection of social and economic inequalities in society. Consequently, higher order factors, rather than mere individual behaviors, fuel the HIV epidemic (Dean \& Fenton, 2010; Kahana et al., 2016; Wohlfeiler \& Ellen, 2007). Social determinants of health may offer some insights into these disproportionate rates (Gant et al., 2014). Another evidence-based argument suggests that structural factors (which constitute $\mathrm{SDOH}$ ) such as living in disadvantaged settings, poverty, unemployment, racial and ethnic segregation, health care, etc., may increase vulnerability to HIV (Kahana et al., 2016).

There is increasing evidence pointing towards a link between social determinants of health and health outcomes including HIV risk (Scott \& Wilson, 2011; Sharpe et al., 2012; Upchurch, Mason, Kusunoki, \& Kriechbaum, 2004; Viner et al., 2012; Willard, Chutuape, Stines, Ellen, \& Interventions, 2012). Yet, HIV interventions do not always adequately address social determinants of health which may influence individual risks. Evidence suggests a link between increased vulnerability to HIV and several SDOH such as living in disadvantaged settings, income, education, housing, poverty, unemployment, racial/ethnic segregation, and access to health care services (Adimora \& Auerbach, 2010; Cene et al., 2011; Gant et al., 2014; Kahana et al., 2016; Viner et al., 2012). Similarly, social capital, poverty, and income inequality have been shown as predictors of AIDS rates and other sexually transmitted cases in the U.S. (Holtgrave \& Crosby, 2003).

\section{Poverty}

Poverty is a leading cause of health inequalities (Oldenburg, Perez-Brumer, \& Reisner, 2014; Wagstaff, 2002). There is overwhelming evidence suggesting that poorer individuals are predisposed to ill health and tend to live shorter than their more affluent 
counterparts (Marmot et al., 2010). According to Marmot et al. (2010), people who reside in poorer areas not only have a shorter life span but will often spend their already short lives with a disability. Evidence also suggests that growing up in poverty can have adverse health implications in adulthood. Early childhood poverty has been shown to have detrimental effects on health or productivity in adulthood including type 2 diabetes, arthritis, early/premature death, poorer productivity (earnings and work hours), hypertension, and other cardiovascular diseases (Duncan, Ziol-Guest, \& Kalil, 2010; Raphael, 2011; Ziol-Guest, Duncan, Kalil, \& Boyce, 2012). Study findings revealed that "compared with children whose families had incomes of at least twice the poverty line during their early childhood, poor children complete 2 fewer years of schooling, work 451 fewer hours per year, earn less than half as much, received $\$ 826$ per year more in food stamps as adults, and are more than twice as likely to report poor overall health or high levels of psychological distress" (Duncan et al., 2010, p. 312).

Many African Americans live in poverty. The current poverty rate for the U.S. is $12.7 \%$, and approximately $22.0 \%$ of Black/African Americans live below the poverty line (United States Census Bureau, 2017). In Kentucky, the current poverty rate is at $17.4 \%$. The US Census Bureau's American Community Survey estimates that at the local level (in Jefferson County/Louisville Metro area), about 17.7\% (of 598,062 people) were living below the poverty level at the end of 2016 , and approximately $31.1 \%$ of those persons living in poverty were African Americans (United States Census Bureau, 2016).

The poverty rate is higher among African Americans than any other racial group in the US. This directly or indirectly impacts HIV vulnerability and HIV/AIDS outcomes through limited access to quality resources (Centers for Disease Control and Prevention, 
2018b). "Economic hardships may Figure prominently in increasing the likelihood of contracting infectious diseases. For instance, poverty may limit choices for selection of residential neighborhoods to ones in which HIV/AIDS and STDs cluster" (Sharpe et al., 2012, p. 250). One study examined social determinants of health among Black/African American men with diagnosed HIV from 2005-2009 in 17 U.S. areas. Heterosexual males located in census tract areas with high concentration of people living below the poverty level had high levels of HIV diagnosis (Gant et al., 2014).

Researchers have established a strong relationship between the HIV epidemic and living in poverty-stricken areas of the U.S. Denning and DiNenno (2010) examined HIV prevalence rates among 18-50-year-old heterosexual individuals across 23 impoverished urban areas across the country and found the HIV prevalence rate $(2.1 \%)$ to be 20 times higher than the national HIV prevalence rate $(0.1 \%)$ among heterosexuals. The researchers concluded that there is a generalized epidemic $(>1 \%$ prevalence rates in the general population) in impoverished areas of the U.S. They also found a statistically significant inverse relationship between HIV prevalence rates and all of the socioeconomic status metrics they examined - "education, annual household income, poverty level, employment, and homeless status" (Denning \& DiNenno, 2010)

Furthermore, individuals who are poorer and more disadvantaged may have difficulty accessing HIV preventive and treatment services due to limited affordability of such services. Findings from a robust qualitative study that explored the perspectives of African Americans living in poorly resourced communities with high rates of HIV/AIDS revealed limited access to health services as a barrier to HIV prevention among African American youth in the study (Cene et al., 2011). The authors asserted that concentrated 
poverty often leaves residents no choice but to access care at free public facilities, but this practice may be stigmatizing to such recipients (Cene et al., 2011). Poverty precludes health care uptake. Living in poverty or disadvantaged conditions has been associated with underutilization of HIV prevention and treatment services, HIV/AIDS antiretroviral therapy (ART), as well as missed medical appointments for HIV-related care (Kahana et al., 2016). In an examination of associations between structural characteristics and HIV disease among a sample of 1,891 HIV positive youth and adolescents (69.49\% of which were African American), Kahana et al. (2016) also found that youth living in more disadvantaged areas were less likely to report ART use. In one study, poverty-related experiences (food insecurity and hunger) were shown to predict non-ART adherence (Kalichman \& Grebler, 2010). Some participants in this study had to choose between getting their HIV medication or paying for food.

\section{Access To Health Services}

Evidence suggest that racial and ethnic minorities have less access to health care compared to Whites. This drives the wide gaps in health outcomes observed among racial and ethnic minorities (Alegría, Alvarez, Ishikawa, DiMarzio, \& McPeck, 2016; Fiscella \& Sanders, 2016). Limited access to high-quality health services like HIV prevention (HIV testing and prophylaxis) as well as treatment services influence health outcomes for people at risk for HIV and living with HIV (Centers for Disease Control and Prevention, 2018b). According to the 2017 Ryan White HIV/AIDS Program Annual Client-Level Data Report, African Americans fare worse at several points of the HIV care continuum (various stages of living with HIV from the time of testing/diagnosis, linkage to care and retention in care, prescription and adherence to treatment/medications, and achievement 
of viral suppression [extremely low or undetectable levels of HIV virus in the body]) (Health Resources and Services Administration, 2018, 2019b). One study that examined national HIV testing prevalence among high school students and young adults ages 18-24 years observed testing rates to be decreasing, especially among young African American females (Van Handel, Kann, Olsen, \& Dietz, 2016).

Moreover, HIV testing is highly important for early diagnosis and treatment. HIV testing reduces missed opportunities and lowers transmission rates, saving money in the long run for lifetime treatment of an HIV infected individual (about \$380,000) (Castel et al., 2015). Lack of awareness of HIV status affects the rates of cases in communities, resulting in missed opportunities and delay in getting care. This may worsen transmission to others and widen disparity gaps (Centers for Disease Control and Prevention, 2018b). Early testing ensures early diagnoses so that infected individuals can be linked to care, receive appropriate treatment, and become virally suppressed. Antiretroviral therapy reduces the chances of the virus destroying the infected person's immune system (Sanders et al., 2005). This increases a person's prognosis and greatly reduces the chances of transmission to others (Centers for Disease Control and Prevention, 2018b; Gardner et al., 2011; Sangaramoorthy, Jamison, \& Dyer, 2018).

Viral suppression is contingent upon access to medication. Yet, although retention in care for African Americans is relatively comparable to the national averages for clients in the Ryan White HIV/AIDS program, disparities in viral suppression are apparent (Health Resources and Services Administration, 2018). The CDC reports that although overall ART prescriptions increased over time, a lower proportion of African Americans living with HIV are in treatment and have suppressed viral loads (Centers for Disease 
Control and Prevention, 2018m). A study by Bradley, Mattson, Beer, Huang, and Shouse (2016) revealed that ART prescriptions increased over the course of four years (20092013) from $89 \%$ to $94 \%$. However, although this increase was noted among all racial groups, especially among non-Hispanic African Americans (an 8\% increase, compared to a 3\% increase among Whites and 7\% increase among Hispanics), African American adults still had a statistically significantly $(\mathrm{P}$-value $=0.01)$ lower ART prescription rate (92.9\%) compared to Whites (95.2\%). Bradley et al. (2016) also noted that "in 2013, sustained viral suppression was $46 \%$ lower among 18-29 year olds compared with persons aged 50 years and older and 25\% lower among African Americans compared with Whites" (P6). Similarly, although viral suppression increased for all minority groups from 2010-2017, African Americans consistently had the lowest percentages across all seven years compared to all other racial/ethnic groups (Health Resources and Services Administration, 2019b). Researchers assert that these stark disparities in sustained viral suppression are likely reflective of social determinants of health such as those that impact access to health care (Bradley et al., 2016).

Disparities in health care access experienced by African Americans may be attributed to poverty, lack of insurance, stigma, social and emotional burden associated with a positive HIV status, housing, low health literacy, discriminatory practices such as excessive incarceration of African American men, and unjust zoning laws (Aziz \& Smith, 2011; Centers for Disease Control and Prevention, 2018b; Denning \& DiNenno, 2010; Fiscella \& Sanders, 2016; Health Resources and Services Administration, 2018; Kerr \& Jackson, 2016; Kerr, Valois, Siddiqi, Vanable, \& Carey, 2015; Sangaramoorthy et al., 2018). 


\section{Health Policies and Political Influence}

During his early days in office, President Barack Obama charged the Office of National AIDS Policy with the task of developing the National HIV/AIDS Strategy (White House Office of National AIDS Policy, 2010). This strategy had three main goals: to reduce the number of people who become infected with HIV; to increase access to care and improve health outcomes for people living with HIV; and to reduce HIV-related health disparities (White House Office of National AIDS Policy, 2010). The intent of this strategy was to direct efforts and funding toward reducing the burden of HIV for Americans. The strategy also recommended that efforts be focused on high-risk populations like African Americans and gay, bisexual, and transgender individuals (White House Office of National AIDS Policy, 2010).

In addition to developing the National HIV/AIDS Strategy, the Patient Protection and Affordable Care Act (ACA) was signed into law to improve health care coverage, access, and affordability for Americans (AIDS.gov, 2015). The ACA is said to be "one of the most important pieces of legislation in the fight against HIV/AIDS in our history"(AIDS.gov, 2015). Prior to the ACA, health insurance companies could choose to deny coverage to persons with pre-existing conditions like HIV (AIDS.gov, 2015). There is increased coverage for HIV care and people living with HIV who are too poor to afford private insurance coverage are now eligible for Medicaid coverage without the previous requirement to have an AIDS diagnosis (AIDS.gov, 2015). Additionally, the law encourages a patient-centered medical home model which fosters high quality and 
improved retention in care for persons chronically infected with HIV/AIDS (AIDS.gov, 2015).

\section{Environment}

Factors within the built and social environment of African Americans may impede their ability to access resources needed to reduce HIV/AIDS disparities. The built environment includes buildings, transportation systems, services and public resources (Satcher, Okafor, \& Dill, 2012). Where a person lives may determine the resources that person can or cannot access at a given time. For instance, distance from health services may deter disadvantaged persons who do not live close to such facilities. Additionally, limited transportation can hinder access to HIV testing (Centers for Disease Control and Prevention, 2018b). Housing also affects health outcomes. Among persons living with HIV who either had temporary or unstable housing, retention in care and HIV viral suppression were observed to be consistently low. This implies that housing has serious implications for HIV outcomes (Health Resources and Services Administration, 2018, 2019b).

Research suggests that substandard neighborhood characteristics impact vulnerability and distribution of HIV/AIDS risks and outcomes within populations who are subjected to such poor living conditions (Adimora \& Auerbach, 2010; Buot et al., 2014; Nunn et al., 2014). In one study, findings revealed that census tracts that were predominantly African American within the city had "higher concentrations of HIV/AIDS, crime, poverty, and poorer health outcomes" (Brawner, Reason, Goodman, Schensul, \& Guthrie, 2015, p. 12). Additionally, their findings revealed a trend in high HIV incidence and prevalence corresponding with "social and structural factors, such as 
overcrowding, disadvantage, limited permeability of neighborhood boundaries, and hampered availability and accessibility of health-related resources" (Brawner et al., 2015, p. 13).

Neighborhood or physical environment can and often does influence formation of sexual networks and HIV risk, especially for groups like African Americans, who, due to residential segregation, cluster together or reside in neighborhoods with other African Americans (Lutfi, Trepka, Fennie, Ibanez, \& Gladwin, 2015). Residential segregation has been linked to adverse health outcomes, including sexual risk, HIV risk, and poor survival rates following an AIDS diagnosis, particularly for African Americans (Buot et al., 2014; Do, Frank, \& Iceland, 2017; Fennie, Lutfi, Maddox, Lieb, \& Trepka, 2015; Lutfi et al., 2015). Evidence suggests that geographical location contributes to tight-knit sexual networks, and this may partially explain why most African Americans choose other African Americans as sexual partners (Adimora \& Schoenbach, 2005). A study by Sullivan et al. (2014) identified individual, dyadic/sexual network and community-level differences between African American and White MSM. Some of their findings revealed that African American MSM in the study lived in neighborhoods with high proportions of other African American residents. They were also more likely than White MSM to reside in areas considered to be economically distressed (census tract areas subjected to poverty, high school graduation, unemployment and median income). Another study which examined heterogeneity of HIV prevalence in sexual networks of African American MSM also found high HIV prevalence in sexual networks of HIV-negative MSM compared to their White counterparts (Hernández-Romieu et al., 2015). 


\section{Stigma}

Another barrier to HIV prevention efforts is stigma — defined as "a mark of shame or discredit" ("Stigma", 2019). Stigma leads to discrimination and marginalization of various groups of persons at heightened risk for HIV (Mahajan et al., 2008; Prado et al., 2013). This is arguably one of the most influential SDOH which greatly impacts HIV disparities among African Americans (Abara, Coleman, Fairchild, Gaddist, \& White, 2015; Centers for Disease Control and Prevention, 2018b; Coleman, Tate, Gaddist, \& White, 2016; Foster \& Gaskins, 2009; Kerr \& Jackson, 2016; Kerr, Valois, DiClemente, et al., 2015). Stigma is often expressed as discrimination towards persons living with HIV and may serve as a barrier to adherence to treatment for persons living with HIV/AIDS (Buseh, Kelber, Hewitt, Stevens, \& Park, 2006; Nyblade, 2006a). Stigma manifests itself in different ways such as attitudes and beliefs of the public, personalized stigma - the fear of being rejected by others if HIV status becomes revealed (Buseh et al., 2006) — or as perceived stigma, experienced stigma, or internalized stigma as described by Nyblade (2006b). As a social determinant of health, stigma plays a significant role in healthseeking behaviors and has been associated with HIV and other health issues (Dean \& Fenton, 2010; Hatzenbuehler, Phelan, \& Link, 2013). For instance, individuals may avoid learning or revealing their HIV status for fear of being shunned or discriminated against by employers, family, and friends (Foster \& Gaskins, 2009; Liu, Canada, Shi, \& Corrigan, 2012).

Addressing stigma among African Americans is extremely important as stigma substantially influences HIV prevention efforts as well as how persons with HIV are 
treated within their respective communities. Fletcher et al. (2016) examined HIV/AIDSrelated stigma among African American women living with HIV in the Southern U.S., where stigma is quite pronounced. Participants in the study reported experiencing stigma at multiple levels (interpersonal, community, and institutional levels). At the interpersonal level, participants reported experiencing stigma in the form of discriminatory attitudes and behaviors towards them from family and friends, following the disclosure of their HIV status. At the community level, participants experienced stigma from interactions within their immediate social circles such as churches or public housing settings, where others within their community treated them badly due to their positive HIV status. At the institutional level, participants reported experiencing stigma in form of exclusion, stigmatization, and discrimination due to policy violations of privacy and confidentiality of persons living with HIV by institutions such as health care systems, pharmacies or places of employment (Fletcher et al., 2016).

Stigma also deters the use of HIV prevention services (Prado et al., 2013). A study by Eaton, Driffin, Kegler, et al. (2015) that examined how stigma and other determinants affect care engagement among African American MSM found that of the 544 African American MSM participants, 29\% reported experiencing stigma from health care providers with regards to their race or sexual orientation. Consequently, health carerelated stigma was associated with longer gaps in time since last medical exam for HIVnegative African American MSM participants and longer gaps in time since last HIV care appointment for HIV-positive African American MSM in the study.

Further, stigma also affects uptake of biomedical interventions such as HIV PrEP (Calabrese \& Underhill, 2015; Herron, 2016). Eaton, Kalichman, et al. (2017) found a 
strong association between stigma beliefs and a lack of interest in using PrEP among men and transgender women who have sex with men in a large southeastern U.S. city. Many participants in the study believed PrEP was for promiscuous persons. In another study with gay, bisexual men and other MSM at heightened risk for HIV, participants expressed that anti-gay and HIV-related stigma were a deterrent to seeking HIV PrEP or other HIV prevention services (Cahill et al., 2017). Similarly, in an ethnographic study which examined factors influencing PrEP adherence among African American MSM, Garcia et al. (2016) found that stigma negatively impacted perceived self-efficacy or community efficacy to adhere to PrEP. This underscores the importance of more innovative, much broader, more comprehensive, multi-level approaches to address social and structural determinants influencing HIV risk (Baral et al., 2013; DiClemente et al., 2007; Wohlfeiler \& Ellen, 2007).

\section{A Brief Overview of Biomedical Interventions and Background to PrEP}

Over the years, traditional behavioral or individual-focused prevention efforts alone have not adequately impacted the HIV epidemic at population levels. Therefore, researchers have considered more radical options to address the HIV epidemic.

Combining behavioral interventions with biomedical approaches was conceptualized as an approach with great potential to end the epidemic. This is expected to greatly reduce HIV acquisition in individuals with heightened HIV risk.

Multiple medical/prevention trials confirmed efficacy of early initiation of ART in reducing risk of sexual HIV transmission among serodiscordant couples. An efficacy rate as high as $96 \%$ reduction in HIV risk was observed in partners who initiated early ART (Cohen et al., 2011; Cohen, Smith, et al., 2013). Efficacy of PEP has been 
established mainly in animal studies (Black, 1997; Otten et al., 2000). Only a couple of case-control studies established efficacy in humans (Cardo et al., 1997; Centers for Disease Control and Prevention, 1995). In 2005, PEP was recommended for use in individuals exposed to HIV either from work-related exposures or through practicing of high-risk behaviors (Smith et al., 2005). PEP has been shown to be useful for preventing HIV in both cases of occupation-related and non-occupation-related exposures to HIV(Mayer \& Venkatesh, 2010; Rey, 2011). However, it's uptake has not been widespread (especially in cases of non-occupational exposures) due to various challenges including the time-sensitive nature of the intervention which requires a potential user to know, beforehand, where and how to access it (Cohen, Liu, et al., 2013). Researchers have also argued that PEP is not likely to make a major impact on the HIV epidemic at population levels given the reactive, rather than proactive, nature of PEP as well as the difficulty associated with ascertaining exposures deemed high enough risk for PEP initiation (Schechter et al., 2004). Researchers believe that lessons learned from PEP delivery could inform the implementation of PrEP in reducing HIV risk in HIV-negative individuals at heightened risk of HIV (Cohen, Liu, et al., 2013). PEP uptake is marginal, particularly in non-occupational cases (known as nPEP). Thus, researchers believe that PrEP would be well-suited for persons engaging in high risk behaviors who are indicated for nPEP (Cohen, Liu, et al., 2013).

\section{Summary of PrEP Clinical Trials/Demonstration Studies}

In addition to PEP, PrEP was conceptualized in anticipation of a foreseeable end to the HIV epidemic. In 2012, the FDA approved Truvada for daily use as a HIV preexposure prophylaxis for HIV prevention in populations at heightened risk of becoming 
infected with HIV (US Food and Drug Administration, 2012). Truvada is a combination of emtricitabine and tenofovir disoproxil fumarate (FTC-TDF), but other PrEP medications may contain only either TDF or FTC (Lehman et al., 2015). Both the combination as well as the monotherapy PrEP options have proven effective in decreasing the rate of HIV acquisition in various high-risk populations including persons who inject drugs, MSM, transgender women who have sex with men, heterosexual men and women, and heterosexual serodiscordant couples (Baeten \& McCormack, 2016; Choopanya et al., 2013; Dolling et al., 2016; Grant et al., 2014; Grant et al., 2010; McCormack, Dunn, Desai, Dolling, Gafos, Gilson, Sullivan, Clarke, Reeves, \& Schembri, 2016).

\section{PrEP Efficacy Studies among MSM and/or Transgender Women}

The iPrEx study, a randomized control trial, was conducted with 2,499 HIVseronegative MSM and transgender women between the ages of 18-67 years old in 11 sites across six countries over the course of 2.8 years. In the study, PrEP was shown to reduce HIV incidence by $44 \%$ overall and by $92 \%$ for those in the control group who were adherent to the drug (they were consistent in taking the daily oral PrEP agent combination drug [FTC-TDF] and had detectable levels of medicine in their blood) (Grant et al., 2010). Similarly, the PROUD study — an open-label randomized control trial - examined real world effect of PrEP among gay and other MSM in England between November 2012 to April 2014. They found that PrEP was highly effective in reducing HIV risk even more so in the real world than in placebo trials. The study also revealed that the use of PrEP did not increase risk compensation (the tendency to engage 
in riskier sexual behaviors if perceived to be protected by PrEP) (McCormack, Dunn, Desai, Dolling, Gafos, Gilson, Sullivan, Clarke, Reeves, \& Schembri, 2016).

\section{PrEP Trials among Heterosexual Serodiscordant Couples}

PrEP trials have been conducted among heterosexual men and women as well. A randomized control study examined efficacy of combination PrEP agent TDF-FTC and single agent TDF in a sample of 4,747 heterosexual serodiscordant couples enrolled in the study and randomized into three groups (placebo, TDF, or TDF-FTC), in nine sites in Kenya and Uganda, between 2008 to 2010 (Baeten et al., 2012). In the study, efficacy of both medications in reducing HIV was observed among both men and women; HIV reduction rates of $67 \%$ and $75 \%$, were observed in the TDF and TDF-FTC groups, respectively, relative to the placebo group. Although there were no statistically significant differences in efficacy rates of TDF or combination TDF- FTC between men and women, in the TDF group, men and women saw a $63 \%$ and $71 \%$ reduction rates, respectively, while in the TDF-FTC group, the HIV acquisition reduction rates were $84 \%$ and $66 \%$ for men and women, respectively. Additionally, for those with detectible levels of medicine in their blood, $86 \%$ and $90 \%$ reduction in rates of acquiring HIV were observed for the TDF and TDF- FTC groups, respectively.

\section{PrEP Efficacy Studies among Persons Who Inject Drugs (PWID)}

Choopanya et al. (2013) assessed PrEP efficacy in reducing HIV risk in a

randomized, double blind, placebo-controlled study conducted between 2005 and 2010 in Bangkok, Thailand. The study included 20-60-year-old individuals recruited from 17 drug treatment centers and reporting injection drug use in the year prior to the study. Not only was PrEP found to be effective in decreasing HIV acquisition risk at $48.9 \%$ in the 
study, but a strong decrease in high-risk injection behaviors was also observed among participants. For instance, injection drug use within the past 3 months among participants decreased from $63 \%$ at enrollment to $23 \% 12$ months into the study, and $18 \%$ by 72 months. Adherence was also observed to be high ( $83.8 \%$ of days of taking study drug) among participants, and women as well as persons aged 40 years and older had better adherence in the study.

While PrEP has been shown to decrease HIV acquisition risk in various populations, efficacy is contingent upon adherence to medication (Kashuba, Patterson, Dumond, \& Cohen, 2012). Efficacy was shown to increase from $48 \%$ to $74 \%$ in participants with detectable blood levels of the study drug, tenofivir, in the PrEP efficacy trial with PWID (Choopanya et al., 2013). This effect was also observed in another placebo-controlled trial by Donnell et al. (2014), where blood plasma levels of the study drug were measured in the control arms with persons taking TDF or the TDF-FTC combination therapy. Findings revealed high efficacy of PrEP drugs to prevent HIV at $81 \%$ efficacy rate for TDF and $91 \%$ for TDF-FTC groups in persons who had blood plasma concentrations of 40ng/L (high adherence level) (Donnell et al., 2014). Antiviral resistance following PrEP use is possible and has been observed in cases where a person who has actively taken PrEP seroconverts to an HIV-positive status. These are, however, rare and have been reported to be more common in cases with monotherapy such as FTC alone (Lehman et al., 2015).

\section{Examining Factors influencing PrEP Among African Americans}

Research suggests that access to PrEP is paramount for its uptake among various high-risk groups, especially African American groups (Eaton, Driffin, Bauermeister, et 
al., 2015; Lelutiu-Weinberger \& Golub, 2016). However, barriers preclude these target groups from readily accessing PrEP. These barriers include, but are not limited to: lack of awareness or knowledge about PrEP (Eaton, Driffin, Bauermeister, et al., 2015; Eaton, Matthews, et al., 2017); medical mistrust (Cahill et al., 2017; Eaton, Driffin, Kegler, et al., 2015; Eaton et al., 2014; Lelutiu-Weinberger \& Golub, 2016); PrEP-related stigma and conspiracy beliefs (Eaton, Kalichman, et al., 2017; Elopre et al., 2018); structural issues such as where and how to access PrEP; cost; insurance; location/proximity to clinics; transportation issues (Elopre et al., 2017); provider attitudes; provider unwillingness to prescribe PrEP, especially to younger populations (Hart-Cooper, Irwin, \& Scott, 2018; Mullins, Zimet, Lally, \& Kahn, 2016); poor provider-patient communications about sexual practices (Eaton et al., 2014); and limited availability of culturally sensitive PrEP clinics or providers (like gender affirming clinics, which are important for effectively reaching transgender women with PrEP) (Sevelius, Keatley, Calma, \& Arnold, 2016). Understanding factors influencing uptake of PrEP among African American high-risk populations will set a precedent for intervention development to improve PrEP uptake.

\section{PrEP Acceptability}

One of the challenges to PrEP uptake among African Americans is PrEP acceptability. Researchers contend that for any PrEP intervention to be successful (i.e. to attain the goal of widespread PrEP uptake), engagement of high-risk populations, for whom PrEP is highly recommended, is a critical first step (Lelutiu-Weinberger \& Golub, 2016). For populations with a history of oppression and marginalization such as African Americans and sexual minorities, stigma, mistrust of the health care industry, conspiracy 
beliefs, and misconceptions may affect acceptability of or engagement with wellintentioned interventions like PrEP (Eaton, Driffin, Kegler, et al., 2015; Eaton et al., 2014; Eaton, Kalichman, et al., 2017; Mutchler et al., 2015).

Race-based medical mistrust has been found to be a strong predictor of health care engagement (Eaton, Driffin, Kegler, et al., 2015) and willingness to engage in PrEP use among African American MSM (Eaton et al., 2014). Due to the lack of trust of medical professionals of other races, some high-risk groups like African American MSM expressed discomfort with talking to their providers about sex, let alone PrEP. This poses a barrier to effective client engagement for HIV prevention. Other concerns affecting interest in PrEP have been reported among young African American MSM, which include fears about PrEP safety, side effects, and toxicity as well as negative misconceptions from peers who may assume that a person on PrEP must be HIV positive (Mutchler et al., 2015).

\section{PrEP Accessibility}

A lack of comprehensive access is slowing down HIV prevention efforts, especially among high-risk populations (Lelutiu-Weinberger \& Golub, 2016). Finding a PrEP provider may serve as a barrier to PrEP access for African Americans with a history of limited access to other health care services. Locally, in Louisville, KY, efforts have been made to inform the implementation of a dedicated PrEP clinic in the city (Muvuka et al., 2016). There are also several PrEP providers within a 50-mile radius of Louisville who can be located using a PrEP finder tool on the website of a local AIDS service organization serving Louisville Metro area (Volunteers of America, 2018). PrEP 
accessibility is also expected to increase as more providers become more knowledgeable about PrEP and more willing to participate in PrEP delivery (Hart-Cooper et al., 2018).

Evidence suggests that identifying and lowering access-related barriers among African American high-risk groups is likely to improve PrEP uptake among them. Researchers acknowledge that, "affordability of medication, laboratory testing, and clinical care visits required for the safe prescription and monitoring of PrEP is a critical issue for its access by those who would benefit from its use" (Smith, Van, \& Huggins, 2017 , p. 2). PrEP is very expensive for consumers. The cost of Truvada is about $\$ 1,300$ per month (Heitz, 2014). Evidence also suggests, based on 2015 estimates, that "the estimated annual cost of PrEP medications and care per person was $\$ 12,913$ for MSM, $\$ 11,711$ for heterosexual and PWID females, and \$11,694 for heterosexual and PWID males" (Smith et al., 2017, p. 5). Medication expense and the inability to pay may pose a barrier to low-income individuals, many of whom may be African Americans, interested in this intervention. To address this barrier, prescription medication assistance programs exist to help clients pay for PrEP (Heitz, 2014). Gilead's U.S. Advancing Access program covers up to $\$ 200$ per month of the client's co-payment and has no income restrictions (Heitz, 2014). Gilead may also cover the full cost of the drug for some uninsured or underinsured patients, based on certain eligibility criteria Levitt (2014). Additionally, the Patient Access Network (PAN) Foundation provides assistance for medications such as Truvada for insured patients who fall at or below 500 percent of the federal poverty line and may cover up to $\$ 7,500$ per year in medication copay cost (Patient Access Network Foundation, 2016). A study by Smith et al. (2017) examined the extent to which PrEP financial assistance needs are either met or unmet nationally for 
covering cost of PrEP medication, clinical visits, or laboratory costs across various highrisk gropus. "Of persons estimated to have indications for its use, $75 \%$ of MSM, $76 \%$ of Het females, $71 \%$ of Het males, and 50\% of PWID have public or private insurance to cover most PrEP care costs" (Smith et al., 2017, p. 6). However, some persons are in need of financial assistance for medication alone (less than 1\%) and for both medication and PrEP-related care (7\%) in each risk category. Researchers suggest that addressing this unmet financial need warrants policy initiatives geared towards more accommodating eligibility criteria for insurance as well as medication assistance programs.

Many insurance plans cover the cost of PrEP. For instance, some states have approved Truvada to be covered for PrEP through Medicaid fee-for-service drug formulary. The state of New York is an example of one of those states in which Medicaid covers PrEP. This initiative, which served to remove financial barriers to PrEP access, was shown to considerably increase PrEP prescriptions filled by Medicaid beneficiaries (Laufer et al. (2015). In this study, the researchers reported a 17\% increase in PrEP prescriptions filled by Medicaid beneficiaries over a three-year period (from 2012-2015). The proportion of African American recipients filling PrEP prescriptions increased substantially by $67.3 \%$ over that period (Laufer et al., 2015).

\section{AIDS Service Organizations' Role in Increasing PrEP Uptake}

AIDS service organizations (ASOs), who are also community-based organizations (CBOs) providing HIV/AIDS services, have a critical role to play in advancing PrEP because of their work providing HIV services within the community. ASOs have the potential to reach out to and engage underserved populations with PrEP education and to provide tailored outreach to these groups. Service organizations have 
been shown to improve PrEP uptake by partnering with academics in community-focused initiatives such as PrEP awareness campaigns (Collier, Colarossi, \& Sanders, 2017). ASOs may also influence PrEP uptake by integrating PrEP promotion within existing HIV/STD prevention services such as HIV testing initiatives (Ayala et al., 2013). Integration of biological interventions such as PrEP into existing organizations' programs could facilitate reach and foster sustainability as well as set precedent for scale-up of interventions like PrEP (Wingood, Rubtsova, DiClemente, Metzger, \& Blank, 2013).

Positive relationships between service providers and priority groups present a great opportunity for promoting PrEP to high-risk populations (Flash, Dale, \& Krakower, 2017). ASOs have been shown to have great potential to improve PrEP uptake through strategic PrEP outreach and delivery services and their interaction with priority groups (cite). Researchers examined the extent to which health care providers and CBOs influence PrEP awareness among priority populations, namely MSM. Seeing a health care provider, getting tested, and receiving condoms from an HIV/AIDS-focused CBO (that is an ASO) were significantly associated with PrEP awareness in the study (Raifman, Flynn, \& German, 2017). Despite this prime opportunity, very few CBOs are adequately engaging with PrEP. In a national sample of 175 CBOs, Smith et al. (2016) assessed $\mathrm{CBO}$ opinions regarding behavioral prevention interventions. Most CBOs are aware of biomedical interventions including PrEP. However, few (13\%) CBOs reported meeting client PrEP needs, although 64\% of CBOs are willing to expand these efforts in the future. CBOs in clinical settings were also more likely than non-clinical CBOs to support expansion of biomedical prevention initiatives (Smith et al., 2016). 


\section{Gaps in the Literature and Rationale for the Study}

HIV PrEP is one of the CDC's high impact biomedical interventions for HIV prevention among priority high-risk populations. PrEP has been proven to be safe and

effective in decreasing the number of new HIV cases in various high-risk groups (Baeten \& McCormack, 2016; Dolling et al., 2016; Grant et al., 2010; McCormack et al., 2016;

Volk et al., 2015), yet uptake remains low among African American groups (Eaton, Driffin, Bauermeister, Smith, \& Conway-Washington, 2015). A recent report on PrEPuse data indicates substantial disparities among African Americans. Of all the 78,360 PrEP perceptions filled in 2016, only $11.2 \%$ were filled by African Americans compared to $68.7 \%$ filled by Whites (Huang et al., 2018). This underscores the importance of scaling up PrEP-use among all African American high-risk groups, with the hopes of narrowing the disparity gaps among them.

Some studies exploring barriers to PrEP uptake among certain high-risk groups have included African Americans as a fraction of their study population (Cahill et al., 2017; Eaton, Kalichman, et al., 2017; Elopre et al., 2017; Lelutiu-Weinberger \& Golub, 2016). A few other studies target African Americans, but most of these studies mainly include African American MSM (Eaton, Driffin, Bauermeister, et al., 2015; Eaton, Driffin, Kegler, et al., 2015; Eaton et al., 2014; Mutchler et al., 2015; Philbin et al., 2016) and in fewer instances, African American transgender women (Eaton, Kalichman, et al., 2017; Eaton, Matthews, et al., 2017). While this is important, given that African American MSM are at much higher risk for HIV, it is also important to include the other sub-groups who, like MSM, are at heightened HIV risk and contribute to the widening disparity gaps. Incidentally, there is a paucity of studies targeting multiple African 
American high-risk groups (African American women, serodiscordant couples and other high-risk heterosexuals, besides MSM and transgender persons, and even fewer studies specifically target African American high-risk youth groups within these contexts (Mutchler et al., 2015). This dissertation intends to address these gaps and add to the knowledge base by including multiple African American risk groups, not only MSM, as well as focusing on youth groups (specifically 18-29-year-olds). Exploring the facilitators and unique challenges to PrEP engagement among the various African American priority high-risk groups is expected to elucidate the factors influencing low PrEP uptake among these groups.

Additionally, ASOs may serve as the bridge between biomedical/research advances such as PrEP and the community as these agencies often serve populations that may be at heightened risk for HIV and indicated for PrEP. Evidence suggests that when researchers collaborate with service agencies, adaptation of research-based interventions is more successful (Kelly et al., 2000; Owczarzak \& Dickson-Gomez, 2011). Despite this recognition of ASO's important role in HIV prevention, there is a dearth of research assessing recommended strategies for ASOs to scale up PrEP. Evidence suggests that few CBOs are adequately engaging with PrEP or may be ill equipped to do so effectively (Smith et al., 2016). More specifically, strategies for ASOs to effectively reach and engage with hard-to-reach African American groups at heightened risk of acquiring HIV either remain understudied or are sparsely published in the literature. This dissertation intends to fill this knowledge gap in the literature by involving ASOs in the study to assess strategies for scaling up PrEP outreach/delivery. 
Moreover, research suggests that involving ASOs in research-based interventions for HIV prevention holds great potential in translating such interventions into practice (Dworkin, Pinto, Hunter, Rapkin, \& Remien, 2008; Kelly et al., 2000; Owczarzak \& Dickson-Gomez, 2011). However, research findings often are not implemented due to the inability of organizations to access or translate such findings within their respective realms of practice. Kelly et al. (2000) contend that HIV prevention research alone is insufficient for curbing the HIV epidemic unless findings are effectively disseminated to service organizations providing prevention services, who are in turn able to effectively implement such interventions by tailoring them to community needs. While many evidence-based interventions have been developed by researchers in academic institutions and disseminated in scholarly journals, this format and language may not be easily understood by community-based organizations (Centers for Disease Control and Prevention, 2018g). Researchers contend that "if effective public health programs, products, and practices are not widely and effectively disseminated, they will not achieve their potential impact to improve the public's health" (Glanz, Rimer, \& Viswanath, 2008, p. 314). Thus, research findings ought to be disseminated to ASOs in a format that is accessible, as well as easy to follow and implement. This dissertation attempts to fill this gap by ensuring that findings of effective strategies for improving PrEP delivery and outreach to African American groups at high risk of HIV are disseminated to ASOs in Louisville, KY in an easily accessible format to aid in their efforts to scale up PrEP outreach and service delivery among African American high-risk groups.

Approaching this dissertation from two angles was necessary, namely examining the perspectives of priority groups regarding factors impacting low PrEP engagement 
among them as well as exploring effective strategies for ASOs to improve PrEP outreach/delivery to priority groups. Addressing wide disparity gaps in HIV rates among African Americans warrants a shift from behavior-centered-only approaches to more ecological approaches. Kurth, Celum, Baeten, Vermund, and Wasserheit (2011) argue that to effectively address the HIV epidemic at population levels, interventions will need to employ combination packages which incorporate multiple approaches (including behavioral, biomedical, social, and structural) at various levels of influence (individual, interpersonal, community, and societal). Employing a multi-faceted approach to understanding low PrEP uptake among African Americans entails examining barriers and facilitators that may act at multiple levels of influence. This is expected to enhance the identification of effective strategies for improving PrEP use among African American high-risk groups and potentially narrowing disparity gaps among them. This dissertation employs an ecological framework to examine factors influencing PrEP uptake at multiple levels, including individual, intrapersonal, organizational, community, and policy.

\section{Theoretical Framework}

This dissertation is guided by three theoretical frameworks: 1) symbolic interactionism and pragmatism (which are the theoretical underpinnings of constructivist grounded theory methods) 2) Social Ecological Model (SEM), and 3) Theory of Reasoned Action/Theory of Planned Behavior (TRA/TPB). Constructs from the second and third deductive theories are utilized only in part one of the dissertation and were only used as theoretical sensitizing concepts to aid in exploring individual, sociocultural, and structural factors impacting low PrEP engagement and uptake among African American groups. 
In this dissertation, the SEM is utilized as sensitizing tools for questionnaire development and as an overarching framework to assess multiple (individual, sociocultural, and structural) factors influencing low PrEP uptake at multiple levels of influence. Constructs from other supporting theories (TRA/TPB) were combined with the SEM and explored within its context for further elicitation of individual-level characteristics shaping the way African American high-risk groups respond to PrEP for HIV prevention. Combining individual behavioral theories with theories which operate at multiple levels helps to achieve a substantial level of effectiveness; this process of combining theories is common in health behavior change interventions (Glanz \& Bishop, 2010). The SEM framework served as a sensitizing tool for developing the study questionnaire to capture factors influencing PrEP uptake at multiple levels — the individual, intrapersonal, organizational, community, and policy-levels.

An ecological approach to this study is imperative because health, disease, and disability are influenced by factors beyond the control of the individual. Consequently, public health researchers contend that individual behaviors do not exist in isolation but are, rather, intertwined or influenced by other factors within the social and physical environments which may restrain or promote behaviors (DiClemente et al., 2007). Ecological approaches, which situate behavior within the contexts of the social and physical environments, are therefore more efficacious for effecting long-term behavior change such as is needed in STI/HIV prevention science (Baral et al., 2013; DiClemente et al., 2007). Thus, the SEM framework is appropriate for this study because it provides the foundation for a multi-level approach to examining the multiple factors imbedded within the complex contexts of both the social and physical environments of African 
American high-risk groups, which may impact their response to PrEP intervention for HIV prevention. In addition to SEM, constructs from TRA/TPB were also utilized during questionnaire development to further elicit nuanced discussions regarding intrapersonal factors that may impact PrEP engagement and PrEP-use decisions among participants.

\section{Symbolic Interactionism and Pragmatism}

Part two of the dissertation employed a constructivist grounded theory approach (CGT). CGT method (Charmaz, 2014) has its philosophical underpinnings in symbolic interactionism (SI) (Blumer, 1986) and pragmatism (Lewis, 1976). SI assumes that meanings and actions are formed and shaped by language and symbols, and there is reciprocal relationship between actions and meaning (Blumer, 1986; Charmaz, 2014). That is, people act towards any given situation based on the how they interpret it, and the meanings ascribed to the situation may in turn be modified by the situation (experiences and encounters) (Blumer, 1986; Charmaz, 2014).

CGT evolved from grounded theory (GT) (Glaser \& Strauss, 1967), but differs slightly from traditional GT in that CGT takes a strong stance on social constructivist interpretive worldview "to acknowledge subjectivity and researcher involvement in the construction and interpretation of data..." (Charmaz, 2014, p. 14). Grounded theory has been defined as " a qualitative research design in which the inquirer generates a general explanation (a theory) of a process, an action, or an interaction shaped by the views of a large number of participants" (Creswell, 2013, p. 83). This approach to qualitative inquiry was originally conceptualized by Glaser and Strauss (1967). Grounded theory entails a systematic approach to gathering and analyzing data in an inductive and iterative manner that allows concepts and themes to emerge from data for theory development 
(Strauss \& Corbin, 1994). Grounded theory is recommended when theories are lacking to explain or understand a particular phenomenon (Creswell, 2013). One central tenet and advantage of grounded theory is to ensure the use of multiple perspectives or multiple voices when collecting and interpreting data to yield theory development (Salazar, Crosby, \& DiClemente, 2015). Proponents of this approach insist that interpretations of data must include the perspectives and voices of those who are being studied (Charmaz, 2014; Strauss \& Corbin, 1994). This serves to reduce bias from the researcher and ensures that data gets presented in a way that accurately represents the perspectives of the target audience, rather than the beliefs, perceptions, or opinions of the researcher. A major advantage of grounded theory is the level to which the approach ensures rigor, depth, and richness of qualitative inquiry through a systematic process of data collection and interpretation, which 'grounds' theory in the experiences of study participants (Charmaz, 2006, 2014; Creswell, 2013; Glaser \& Strauss, 1967; Strauss \& Corbin, 1994). This approach was useful for exploring strategies for ASOs who work with African American high-risk groups to enhance PrEP outreach/delivery and to potentially scale up PrEP-uptake among these groups. Thus, this study assumed that ASOs will approach PrEP implementation based on the meanings they ascribe to it and their interpretations of PrEP implementation may be impacted by their unique experiences providing PrEP outreach with African American priority groups. This also helped to determine the range of meanings of PrEP interpretation held by various participants and the corresponding actions (strategies) that were informed by those meanings. This research data derived from this type of approach forms the basis for context-specific frameworks for explaining processes embedded within these social interactions. 
Consequently, I anticipated that concepts would emerge from the data to inform the development of a PrEP outreach and engagement context specific framework. This framework is expected to guide intervention development for ASOs in the scaling up of PrEP outreach and engagement of priority populations to ultimately increase PrEP uptake among these groups.

\section{Social Ecological Model (SEM)}

The SEM provides the basis for observing factors affecting health outcomes at multiple levels of influence (Figure 7). The SEM was conceptualized based on the premise that physical, social, and environmental influences may play a pivotal role in health and disease outcomes among individuals (Stokols, 1992). Proponents of the ecological perspective assert that there is a reciprocal relationship between behavior and the environment (McLeroy, Bibeau, Steckler, \& Glanz, 1988; Runyan, DeVellis, DeVellis, \& Hochbaum, 1982; Stokols, 1996). That is, behavior can be shaped by and can shape the social and physical environment. Also, behavior is affected by and can affect multiple levels of influence; multiple levels of influence, in turn, can and do affect behavior (McLeroy et al., 1988; Stokols, 1996). The SEM recognizes that individuals are part of and influenced by their larger environments and social systems; also, health outcomes or behaviors of individuals are influenced by several factors existing at multiple dimensions or multiple levels within those environmental contexts (Glanz \& Bishop, 2010; Glanz et al., 2008). As applied in health promotion, the SEM consists of five levels: individual or intrapersonal (e.g. attitudes and knowledge, behavior control), interpersonal (family, peer groups, sexual networks), institutional or organizational (e.g. cost, provider access, access to health care), community or societal (e.g. cultural beliefs, 
myths, stigma, homophobia, discrimination, etc.), and public policy level, with the understanding that factors may overlap across these levels (Golden \& Earp, 2012; McLeroy et al., 1988).

The first level of the SEM is the individual level. These are biological or behavioral factors influencing health outcomes of individuals. Characteristics associated with the individual level include health status or developmental history, knowledge, attitudes, behaviors, perceptions, skills, etc. (McLeroy et al., 1988). The individual level of the SEM framework is applied in this dissertation to explore how individual characteristics within the environment of African American youth at high risk of HIV may inform their decision to either engage or not with PrEP for HIV prevention.

Figure 7 The Social Ecological Model.

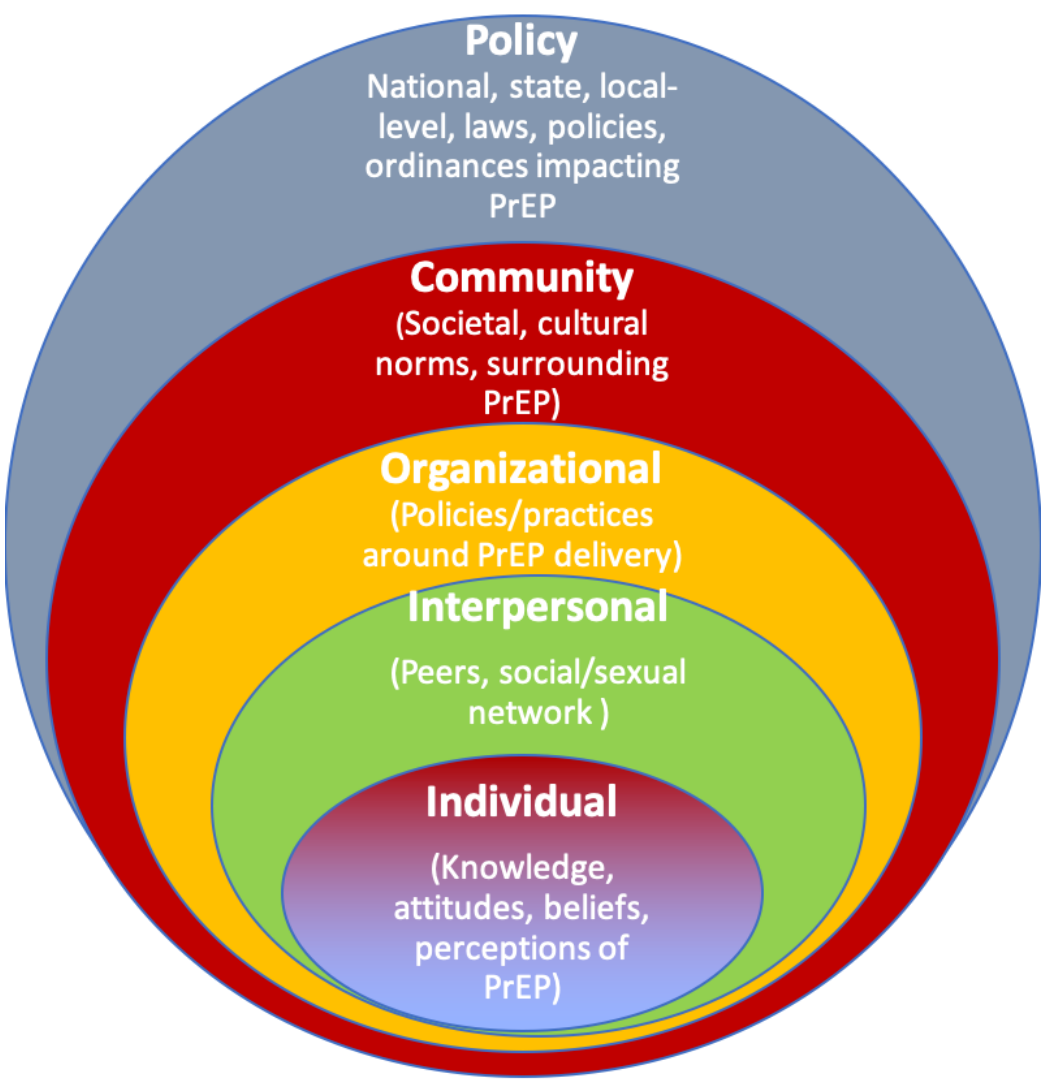


Individual-level characteristics explored, which may influence participants attitudes towards PrEP use, include PrEP knowledge, awareness, self-efficacy to use PrEP, perceptions of PrEP use and PrEP access, perceived barriers, and facilitators towards PrEP use. Previous studies have assessed individual factors affecting PrEP engagement (Crosby et al., 2014; Eaton, Driffin, Bauermeister, et al., 2015).

The second level of the SEM, the interpersonal level/group level, goes beyond the individual factors to include support systems and networks (like peer groups, family, sexual networks etc.) which have an impact on individual behaviors. "Interpersonal relationships with family members, friends, neighbors, contacts at work, and acquaintances are important sources of influence in the health related behaviors of individuals" (McLeroy et al., 1988, p. 356). In this dissertation, barriers and facilitators to PrEP engagement assessed include how participants' interactions with their peers, relations, social or sexual networks influence PrEP engagement decisions.

The third level — organizational level of the SEM — includes factors like formal or informal rules, regulations, and policies within an organization which may influence behavior change. These characteristics are important as institutions or organizations can play a vital role in health outcomes of individuals. Organizations may serve as targets for spearheading activities and diffusing health promotion programs (McLeroy et al., 1988). This level of the SEM is applied in this dissertation by exploring how organizational practices or policies within an ASO may be a barrier or facilitator of PrEP uptake for African American high-risk groups. Examples will include current ASO practices such as PrEP outreach and PrEP promotion approaches (campaign strategies and targeted messaging) or the lack thereof towards African Americans within various risk groups, 
and how these practices affect the response of these priority groups to PrEP. For instance, if ASOs within the community lack cultural awareness and are not tailoring interventions to African Americans or being inclusive of all risk groups, PrEP promotion campaign interventions may not be effective for stimulating PrEP use among this population. PrEP promotion campaign and/or commercial messages, depending on how they are framed, may either facilitate or deter PrEP use for some high-risk groups.

At the fourth level of the SEM, the community level, factors involve social, cultural, and societal norms and may leverage relationships among organizations and institutions for influencing behavior (Baral et al., 2013). Although there are many definitions of community, for the purpose of the SEM, McLeroy et al. (1988) define community in terms of relationships. Their definitions include a) considering community in terms of mediating structures such as "family, informal social networks, churches, voluntary associations, and neighborhoods" to which individuals may belong (p.363); b) defining community in terms of relationships among organizations within geopolitical settings, which often influence the behavioral outcomes of the individuals within a community; and c) looking at community in terms of the role power structures play in defining community health problems by way of controlling how resources are allocated within various communities. While this dissertation does not seek to directly explore factors influencing PrEP engagement at this level, we recognize that data may reflect community level influences to PrEP engagement that may be reported by participants in the study. These factors, such as medical mistrust and health care settings as they influence PrEP use among high-risk groups, have been previously reported (Philbin et al., 2016). 
The fifth and final level of the SEM is the policy level. Characteristics of this level include local, state, and national level policies, laws, and ordinances designed to protect the health of communities (McLeroy et al., 1988). Interventions at this level often address structural determinants of health, such as environmental factors, organizational and institutional policies, programs, and practices, as well as legislative and regulatory approaches (Golden \& Earp, 2012). In this dissertation, policies impacting PrEP engagement will be explored with ASOs.

SEM has been recommended to guide development and implementation of health promotion programs and previous research studies including HIV intervention. Studies have employed various levels or combination of levels of the SEM (Baral et al., 2013; Eaton, Driffin, Bauermeister, et al., 2015; Loosier et al., 2016; Philbin et al., 2016). In one study, researchers modified the SEM to assess multiple layers of HIV risk among vulnerable populations in which they represented HIV risk layers/levels as individual, network, community, policy, and stage of the HIV epidemic (Baral et al., 2013). At the individual level, the researchers looked at biological or behavioral factors associated with acquiring or transmitting HIV; intrapersonal-level risks included social sexual networks such as groups predisposed to exposure to HIV and family and social networks which could provide support or help foster social norms; community-level risks assessed environmental sources of prevention and treatment as well as stigma and discrimination; policy-level risks were described as policies created and implemented with implications to either promote or reduce HIV risks such as policies which criminalize homosexuality and substance abuse prevention interventions like needle exchange programs, methadone treatment initiatives, etc. Finally, at the stage of epidemic level, researchers explained 
that individual risks of HIV acquisition should be interpreted within the context of community HIV incidence or prevalence, since HIV risks are contingent upon the stage of the epidemic within the community. The researchers concluded that the modified SEM model interventions (which situate individual-level risks in the context of networks, community, and public policy) hold better promise in altering the course of the HIV epidemic at population levels (Baral et al., 2013). In another study, Philbin et al. (2016) also assessed factors impacting PrEP use among Black MSM across multiple levels individual, interpersonal, community, and structural levels. They demonstrated that exploring factors influencing low PrEP uptake at more than one level is important for developing effective interventions to successfully address such factors among vulnerable groups like African American MSM.

\section{Theory of Reasoned Action/Theory of Planned Behavior (TRA/TPB)}

The constructs of the TRA/TPB (Ajzen, 1991) aid in the understanding of how PrEP uptake is influenced by individual factors which may serve to either facilitate or deter a behavior or health outcome, in this case PrEP uptake. TRA/TPB posits that attitudes towards a behavior (PrEP uptake), subjective norms (perceived social pressure, stigma around HIV/PrEP), and perceived behavioral control factors (PrEP knowledge, perceived PrEP access, PrEP awareness) predict the performance of a behavior (PrEP use) or, more precisely, influence the intention to perform that behavior (Ajzen, 1991). Based on this theory, if individuals have positive attitudes towards a particular behavior (in this case PrEP use), if they have favorable subjective normative beliefs (that is they believe that their social network or referents approve of PrEP), and perceived control (that is they are aware of PrEP, have PrEP knowledge, have confidence to use PrEP, and 
have the resources to access PrEP), they will be more likely to perform the behavior (Ajzen, 1991).

Figure 8 Adapted conceptual framework of TRA/TPB.

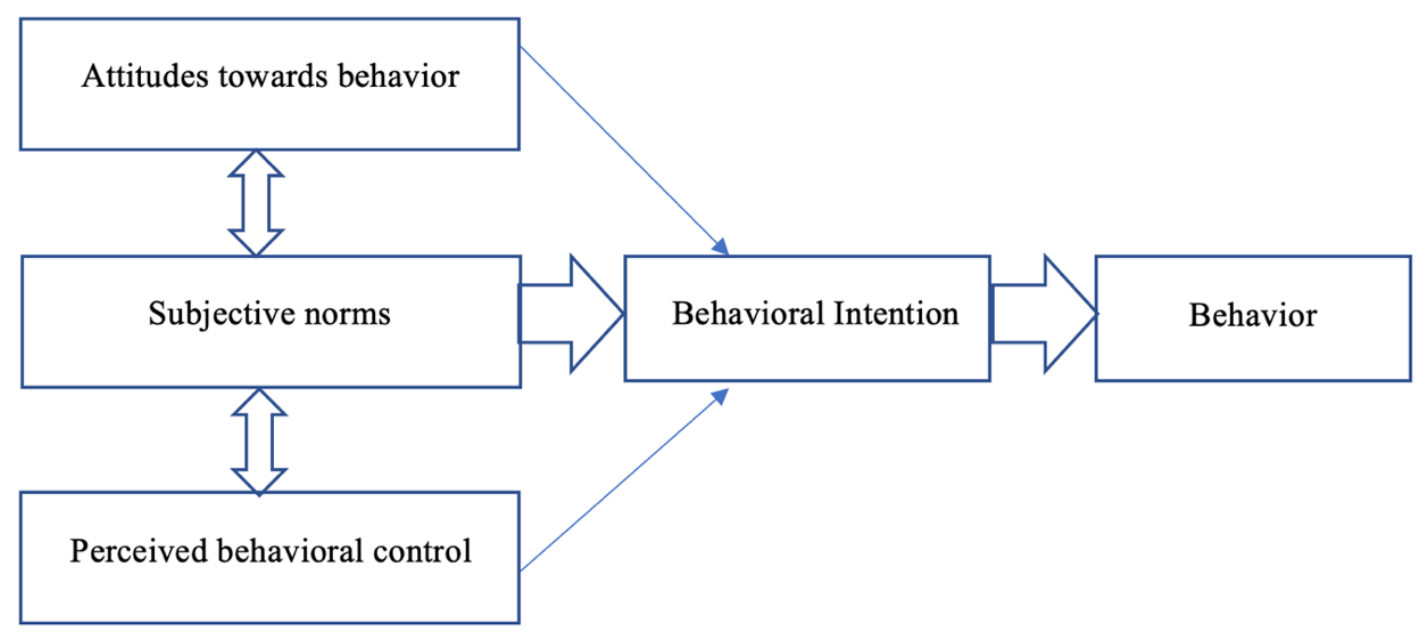

Source: Theory of Planned Behavior (Ajzen, 1991).

\section{Conclusion}

Despite a decline in HIV incidence nationally, African Americans continue to be disproportionately impacted. The literature demonstrates that PrEP holds potential to impact the HIV epidemic. Clinical trials and demonstration projects have established PrEP's safety and efficacy and it has received FDA approval. Yet, its uptake among African American high-risk groups, a population that may need it the most, has been marginal due to the social determinants of health and other factors influencing the acceptability, availability, and accessibility of PrEP. Despite this recognition, there has been limited research geared towards understanding factors influencing PrEP engagement and uptake among various African American high-risk groups.

Moreover, evidence suggests that AIDS service organizations hold the potential for scaling up PrEP awareness and uptake by tailoring PrEP outreach to African 
American high-risk groups. Yet, there are very few studies exploring ASO involvement and strategies for scaling up PrEP especially among various African American groups. This dissertation seeks to add to this body of knowledge by exploring factors impacting low PrEP uptake among multiple African American high-risk groups as well as identifying strategies for ASOs to improve PrEP outreach/ delivery to African American high-risk groups to aid in the scale-up of PrEP uptake among them.

Details of the study, including study design, scope, procedures, and study implications are discussed in the next chapter. 


\section{CHAPTER III METHODOLOGY}

\section{Introduction}

In the previous chapter, I reviewed evidence from previous studies, and identified gaps in the literature. I also provided the rationale behind this current study. To reiterate, this study explored factors (barriers and facilitators) contributing to low PrEP engagement and uptake among various African American priority groups. It also examined strategies for AIDS service organizations (ASOs) to improve PrEP outreach/delivery efforts for scale-up of PrEP among African American high-risk groups. In this chapter I will describe what methods were employed to accomplish this purpose. I also describe and provide justification for the various approaches utilized to answer my research questions. Structurally, this chapter includes seven main sections (with sub-sections as applicable): 1) scope of the proposed study; 2) justification and use of findings; 3) study description; 4) philosophical assumptions and interpretive framework; 5) criteria for establishing rigor and trustworthiness; 6) study limitations; and 7) dissemination of study findings.

\section{Qualitative Research}

Deciding on which method of inquiry to use depends on the nature of the research questions as well as the nature of data needed for that particular research study (Onwuegbuzie \& Leech, 2005; Rich \& Ginsburg, 1999). Quantitative and qualitative inquiry differ in several ways. Quantitative approaches are better suited for addressing 
the research questions of "what", "when", "how many", while the qualitative approach provides the platform for exploring the "how" and "why" as well as the contexts and motivations behind risk behaviors (Rich \& Ginsburg, 1999). Creswell (2013) states that "qualitative research begins with assumptions and the use of interpretive/theoretical frameworks that inform the study of research problems addressing the meaning individuals or groups ascribe to a social or human problem" (2013, p. 44). Whereas the field of quantitative research takes a positivist paradigmatic approach to inquiry, the qualitative research field takes a more interpretivist view to inquiry (Salazar et al., 2015). Positivism focuses on relationships between facts which can be directly observed and verified; that is, it "involves the use of methods that should be objective and involves testing of theories through the generalization and falsification of hypothesis in order to assemble "facts"” (Salazar et al., 2015, p. 210). On the other hand, "interpretivists view the world as a multiplicity of realities in which each individual perceives, understands, experiences, and makes meaning of that reality in different ways; thus reality is socially constructed" (Salazar et al., 2015, p. 211). The quantitative approach also focuses more on assessing relationships and factors as well as stability and generalizability of concepts to larger populations (Yardley, 2000). Rich \& Ginsburg (1999) state that, "while it can indicate, what happens to people from differing demographic groupings, quantitative inquiry lacks the ability to look at the complex interplay among factors that produce individual choice or behavior. Although it cannot look for trends among large groups, qualitative research is an ideal approach to elucidate how a multitude of factors such as individual experience, peer influence, 
culture, or belief interact to form people's perspectives and guide their behavior" (p.327).

Unlike the quantitative approach to inquiry, which focuses on breadth and generalizability of a given concept, qualitative inquiry allows for depth and complexity in understanding of a given issue from the perspective of the participants being studied (Creswell, 2013; Yardley, 2000). This complexity and the level of detail can only be achieved by speaking directly to the people and allowing them to express themselves without any imposition of preconceived information the researcher expects to find or what has been observed in previous literature (Salazar et al., 2015). Consequently, whereas quantitative research is more deductive in nature, involving the testing of prederived hypothesis, qualitative research employs an inductive approach where meaning is derived from interpretation of data which may eventually constitute a hypothesis of theory (Salazar et al., 2015).

Therefore, considering the differences between the two approaches to research, qualitative inquiry is a more appropriate design for this current study. This study focuses on exploring and deriving a deeper and richer understanding of factors precluding African Americans from optimally engaging with PrEP for HIV prevention. The qualitative approach provides the platform for the voices of those most affected to be heard, as individuals share their own stories within the context of their personal experiences (Creswell, 2013). These types of methods also "genuinely offer a complementary set of investigative approaches which can bring fresh insight into health and illness" (Yardley, 2000, p. 216). While previous studies have reported quantitatively on various factors influencing low PrEP uptake among some populations, the experiences 
of young African American high-risk groups with regards to PrEP engagement may differ from those of the general population. Understanding the nuances in attitudes and behaviors or predispositions towards PrEP use among hard-to-reach and marginalized populations would require a more exploratory approach, such as qualitative inquiry. This allows for the elicitation and contextualization of underlying reasons behind low PrEP engagement within this population.

While qualitative inquiry is better suited for exploring a problem and deriving deeper meaning, this process is not without its challenges, which will be taken into consideration. For instance, qualitative research is labor-intensive. Thus, the qualitative researcher must be willing to commit time and resources to field work, complex timeconsuming data analysis processes (which entail sorting through high volumes of data to derive themes), and commit to extensive lengthy writing that must reflect the perspectives of the participants (Creswell, 2013). Another caveat to qualitative inquiry is that this field of research study "does not have firm guidelines or specific procedures and is evolving and constantly changing" (Creswell, 2013, p. 49). Additionally, this methodology is quite complex and requires data reduction, an intricate process of interpreting people's lived experiences in the attempt to arrive at an understanding of their perspectives (Salazar et al., 2015). Thus, to avoid an oversimplification of this process, Salazar et al. (2015) recommend that the researcher should immerse themselves in the study and take an insider's point of view, which is referred to as "emic and should entail using multiple methods such as interviewing, observation, focus groups, and so on" (P. 221). The current study takes these issues into consideration. For instance, the study employed various methods of data collection including focus groups, interviews, field 
notes, etc. Focus groups were conducted with priority populations for whom PrEP is recommended, while in-depth interviews were conducted with key informants from ASOs who have experience conducting HIV prevention (especially PrEP outreach). Emphasis has been placed on ASOs conducting PrEP delivery with African American high-risk groups.

\section{Scope of the Proposed Study}

The proposed study has two parts. A portion of the study (part one) is nested within an existing larger study, the AFYA study (described below). Part one of the proposed study utilizes data from the AFYA study, but varies slightly from the AFYA study's scope and aims. Part two assesses PrEP implementation strategies among AIDS Service Organizations (ASOs) outside of Louisville Kentucky, who have been successful in PrEP service delivery and outreach among various high-risk populations, especially those ASOs who have a history of success with African American high-risk groups. The intent of this is to provide the opportunity for ASOs in Louisville to gain insight into the experiences of other ASOs around the country. The Louisville ASOs can learn from other ASOs about challenges and facilitators to PrEP delivery and recommended practices for engaging high-risk groups (especially African American high-risk groups) for PrEPdelivery. This second part of the study also assessed Louisville ASOs' current strategies as a basis for tailoring recommendations.

\section{Justification and Use of Study Findings}

Understanding reasons for low PrEP use and engagement among African American groups as well as engaging ASOs to improve their PrEP outreach to the various high-risk groups is critical for scaling up PrEP uptake and decreasing the threat of HIV to 
the African American population. The results from this dissertation are expected to inform the improvement of ASOs' PrEP service delivery programing as well as health promotion interventions targeting African American groups for improving PrEP uptake. This dissertation elucidates factors influencing low PrEP uptake among African Americans. There is a particular focus on priority high-risk groups. The study also contributes to the scant literature on PrEP among multiple African American priority groups. Additionally, the findings provide an understanding of preferred and effective outreach methods for reaching African Americans with HIV prevention messages as well as strategic ways for addressing barriers and concerns toward PrEP uptake among various high-risk groups. This dissertation facilitated the identification of pertinent areas and relevant strategies for focusing and tailoring the development of interventions geared towards increasing PrEP implementation among African Americans by ASOs in Louisville/Jefferson County.

\section{Study Description}

This dissertation involved two study designs described below as parts one and two. Data from both studies were utilized to answer the following research questions:

R1. What are the multi-level barriers and facilitators to PrEP engagement, from the perspectives of African American youth groups in Louisville, KY who are at high-risk for HIV?

R2. What are the strategies, nationally, for PrEP outreach and delivery among various high-risk groups, particularly African Americans, from the perspectives of key informants in ASOs across the country? 
R3. How should ASOs in Louisville, KY approach PrEP outreach/delivery with African American groups at high-risk to improve PrEP engagement, based on evidence from research questions 1 (local context) and 2 (national context)?

The study aims in the dissertation included:

A1. To explore and understand barriers and facilitators to, and engagement with, PrEP uptake among priority groups.

A2. To develop a context specific framework grounded in experiences of ASOs of how they have successfully implemented PrEP outreach among African American priority high-risk groups.

A3. To identify and describe effective strategies in the form of recommendations for ASOs in Louisville, KY to improve service delivery and outreach to African American priority high-risk groups for PrEP engagement.

\section{Part One of the Dissertation Study}

\section{A Description of the AFYA study}

A portion of the dissertation utilized data from the AFYA study to answer the first research question (R1). The purpose of the AFYA study was to increase PrEP availability and PrEP use among African Americans with ties to west Louisville (WL; a locale demonstrating the highest HIV rates in Kentucky). The goal was to increase PrEP awareness and access to priority high-risk groups: men who have sex with men [MSM], transgender females, people who inject drugs (PWID), high-risk heterosexuals (sex workers, people with HIV positive partners, individuals demonstrating heightened sexual risk behaviors, non-users of condoms with HIV status unknown partners). AFYA study focuses on African American youth, ages 18-29 years old in high-risk groups, residing in Louisville metro area (with emphasis on west Louisville). 
The intent of the AFYA project is to implement a multi-level (behavioral and structural) intervention to: 1) advance knowledge, willingness to prescribe, and PrEP prescription among health care providers serving west Louisville clients; and 2) increase PrEP uptake among high-risk African American youth (ages 18-29 years old). One of the project's aims is to scale up AIDS service organizations' (ASO) PrEP outreach efforts through educational programming and improving capacity for service delivery around PrEP.

\section{Summary of AFYA study methods applicable to this dissertation study}

The AFYA study utilized a generic qualitative approach to generate data (Merriam \& Tisdell, 2015). Theory development was not a goal for the AFYA study. The project conducted focus groups with priority high-risk groups, many of whom are marginalized. Focus groups are great for special groups and often maximize group dynamics to enrich conversation, as group members stimulate each other (Salazar et al., 2015).

\section{Study Setting}

The research study took place in Louisville/Jefferson county. The University of Louisville facilities served as an on-site location for data collection. This location was chosen because of the study team's access to existing facilities which are convenient and accessible to study participants. One focus group was held in the home of a community organizer.

\section{Inclusion Criteria}

Participants were allowed to participate in the study if they self-identified as an African American, between the ages of 18-29 years old, reside in the Louisville Metro 
area in Kentucky (west or south Louisville), and self-report engagement in high-risk behaviors fitting the description of at least one of these high-risk groups: men who have sex with men (MSM), transgender persons, persons who inject drugs (PWIDs), and other high-risk heterosexual individuals (commercial sex workers and serodiscordant heterosexual couples as well as other heterosexual persons who do not use condoms or have partners with unknown HIV status).

\section{Recruitment}

Recruitment strategies for the AFYA study included the following: partnering with local community-based organizations (CBOs) known to provide HIV prevention services to any of the target high-risk groups (ex. Kristy Love Foundation [an organization that focuses on supporting survivors of sex trafficking]); hiring recruiters to help recruit the hard-to-reach groups (LGBTQ+); conducting outreach at local churches where we set up a table and made an announcement during service; advertisements (print and social media); speaking at meetings for community organizations/neighborhood association meetings (example, Shawnee Neighborhood Association, and the Signature Partnership Council); partnering with organizations to carry out HIV testing to recruit individuals getting tested for HIV; placing or distributing flyers at local CBO offices (House of Ruth); distributing flyers at Syringe Exchange Program (SEP) at Louisville Metro Department of Health and Wellness; recruiting through mobile SEP; recruiting in west Louisville grocery stores and events (with high-risk African Americans and or LGBTQ+ in attendance such as a ball, the Black Magick Festival); recruiting at parties and community meetings; on-site recruiting and distributing of flyers at University of 
Louisville; and focus group participants also spread to social networks through word-ofmouth and by direct referral of peers through the method of respondent-driven sampling.

\section{Sampling}

The AFYA study utilized a type of purposive sampling technique called chain referral sampling, specifically respondent-driven sampling (RDS). RDS begins with an individual (a seed) who is identified based on specific characteristics fitting the purpose of the study. This individual is then asked to refer or nominate another individual with similar characteristics to participate in the study (Trotter II, 2012). Typically, each individual who participates in the study gets to recruit up to three of their peers into the study and receives an incentive for each peer who participates (Heckathorn, 1997). The process is repeated in multiple waves until the desired sample size is reached (Heckathorn, 1997; Trotter II, 2012). This type of chain referral sampling technique facilitates the recruitment of hard-to-reach/hidden populations by incentivizing individuals participating in the study for recruiting other members of their social networks with similar characteristics into the study (Heckathorn, 1997; Trotter II, 2012). RDS is said to be a more valid and reliable technique in recruiting hidden populations since it reduces sampling bias by ensuring a more representative sample through its multiple waves approach, unlike other non-probability sampling techniques (Heckathorn, 1997; Trotter II, 2012). As part of the larger study, which included a survey as well as focus groups, participants representing priority group members were recruited as seeds and offered a small incentive for recruiting up to three other priority group members. 
Participants were asked to complete the demographic survey before recruitment and receive $\$ 10$ for each recruit who completes the survey (up to $\$ 30$ ). This process took about five waves to achieve the desired number of participants to be recruited for the study (approximately 200). Following completion of the survey, participants were assigned a focus group based on the risk category. Not everyone who took the survey participated in the focus groups. A total of 63 individuals who completed the survey also participated in 11 focus groups.

\section{Data Collection}

Informed consent was administered by the AFYA study team to all study participants before focus group data collection commenced. Following informed consent, the research team administered a survey instrument using a computer-based method known as Audio Computer Assisted Self Interviews (ACASI), which presents survey questions and response options through audio and text. Demographic information was also collected through this means. The team utilized password protected, encrypted tablets for this process. The ACASI methodology not only simplifies survey-taking for individuals with low literacy skills, but it also enhances the purity of the data by improving response validity and increasing confidentiality (Morrison-Beedy, Carey, \& $\mathrm{Tu}, 2006)$. The survey took approximately 25 minutes to complete and participants received \$20 for survey completion. Following completion of the survey, some participants were assigned a focus group based on the risk category (not everyone who took the survey was expected to participate in the focus groups). A semi-structured focus group guide (Appendix A) was developed, pilot tested, and administered by members of the study team. Two team members were present in all except two of the 11 focus groups 
conducted. One team member facilitated the focus group, while the other team member took notes. Focus groups lasted approximately 60 minutes. Study participants each received $\$ 35$ for focus group participation.

\section{Audio recording}

A digital audio recorder was utilized for the focus groups. Salazar et.al (2015)

recommend that interviews should be audio-recorded and later transcribed verbatim before analyses. Before starting any recording, the device was inspected to ensure it was functioning properly.

\section{Transcribing Data}

At the end of each focus group, the audio files were transferred from the recording device to a password-protected computer to be transcribed. Transcripts were checked for accuracy by listening to audio recordings to ensure aligning with transcript verbiage. If there was any discrepancy between the transcript and the audio recording, the appropriate corrections were made accordingly. A total of 11 focus groups were transcribed by various study team members and a transcription service (Rev.com). Specifically, I transcribed two focus groups, other team members also transcribed two focus groups, and the remaining seven were transcribed by the transcription service and reviewed by myself and other team members for accuracy.

\section{Data Management}

Identifiable information (names) were removed from any study data collection materials to protect the identities of the participants. All data/documentation (surveys, forms, recordings, and field notes) gathered during field work were stored in a secure 
location not to be shared with anyone who is not part of the research study. Focus group data (audio and transcript files) were uploaded onto a secure, password-protected portion of the University of Louisville server. All other study materials were stored in locked cabinets at the University of Louisville.

\section{Data Analysis Strategy and Process}

\section{Analysis Strategy}

Because theory development was not a goal for the AFYA study, I utilized constructivist grounded theory (CGT) (Charmaz, 2014) analytic techniques for a rigorous, systematic data analysis process to enable themes to evolve from the data, grounded in the experiences of participants as they pertain to PrEP use and engagement.

Merriam (2009) defines data analysis as the process of "making sense out to data", and this process involves "consolidating, reducing, and interpreting what people have said and what the researcher has seen and read — it is the process of making meaning" (pp.175, 176). The data analysis process in this study (Figure 9) was inductive; that is, main concepts and themes emerged from the data (Pope, Ziebland, \& Mays, 2000). Thus, the analysis process utilized principles of constructivist grounded theory (CGT) (Charmaz, 2014). CGT principles include initial coding (line-by-line coding, using gerund [ing words]), focused coding, and memo writing which are known to aid in data synthesis and allow themes and theories to emerge from the data (Charmaz, 2014). Because the goal for this part of the AFYA study was not to ultimately develop theory, I utilized CGT analysis strategies throughout the coding process to enable the description of lived experiences of African American priority groups in the study. Thematic coding was employed to arrive at final categories (Strauss \& Corbin., 2015). A process of 
constant comparison, whereby data is compared against data to refine meaning, was also utilized as part of the analysis process as stipulated by CGT (Charmaz, 2014; Strauss \& Corbin, 1994). Memoing throughout the data analysis process also contributed to finalizing categories and subthemes (Birks, Chapman, \& Francis, 2008; Charmaz, 2014; Strauss \& Corbin, 1994).

Figure 9 African American Youth Focus Groups Analysis Process.

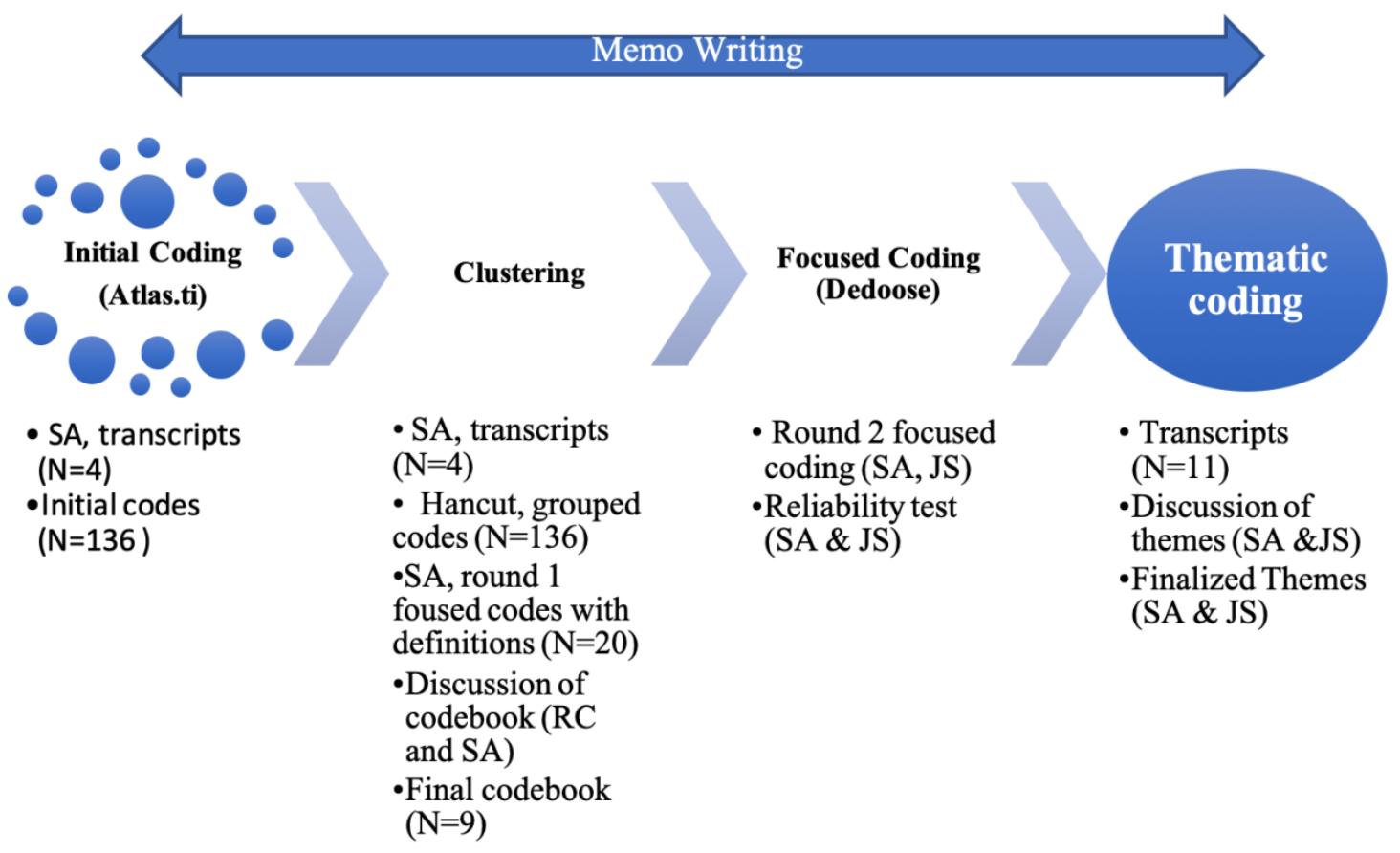

\section{Analysis Process}

Following data collection and transcription, data analysis commenced. I utilized Atlas.ti software for the first round of the data analysis, initial coding, to code one-third of the data ( $\mathrm{N}=4$ focus groups). Atlas.ti is user-friendly interface due to its intuitive nature, ease of use, and visualization (Barry, 1998). Atlas.ti has a wide range of uses including, "the capacity to deal with large amounts of text, as well as the management of annotations, concepts, and complex structures including conceptual relationships that emerge in the process of interpretation" (Muhr, 1991). However, I only chose to use 
Atlas.ti for my initial coding process of the focus group data mainly because it was the only software to which I had access at the time. I needed software to assist with organizing the process of generating initial codes, through a line-by-line process that is extremely daunting to organize by hand. The Atlas.ti software helped to track and sort unique initial codes in alphabetical order. This helped to avoid dealing with code duplicates as would have been the case with hand-coding. For the rest of the analysis, I utilized Dedoose. This was a preferred software over Atlas.ti because Dedoose is a webbased application, which means it can be accessed anywhere, any time, on any devices with internet access (Dedoose.com). This supports teamwork by affording all team members access and control over the same project, while conducting data analysis simultaneously in real time.

Constructivist grounded theory analytic techniques (initial, focused coding, memoing (Charmaz, 2014) and thematic coding (Strauss \& Corbin., 2015) were utilized for a rigorous and systematic data analysis to inductively generate themes (Merriam \& Tisdell, 2015; Strauss \& Corbin., 2015) relating to factors impacting PrEP engagement and uptake among various African American priority groups. Codes (short phrases or words used for explaining and making sense of the data) were derived from the data through an initial line-by-line process to allow codes to emerge from the original data. Four out of 11 transcripts were initially coded and yielded 136 initial codes. These codes were combined in multiple iterations to arrive at a finalized codebook with nine focused codes. The focused codes were clearly defined to highlight the underlying properties of each code. Two members of the team (RC and SA) worked independently and together to refine codes and negotiate code definitions to arrive at a finalized version of the 
codebook (nine focused codes). Some sub-codes (child codes) were derived to help create sub-categories within the main focused codes to provide an in-depth analysis of the data. Two team members (SA and JS) independently applied the final codebook to the entire dataset to ensure consistency in code application and to increase credibility of the process (cite). A pooled kappa score of 0.90 indicated almost perfect inter-rater agreement among coders (McHugh, 2012).

\section{Limitations}

This study had limitations. Due to some participants' preferences to not identify with any specific labels or to identify with more than one category, hence the mixed groups in the study, the number of participants in MSM-only and LGBTQ+-only groups appeared small, compared to the number of heterosexual participants across 11 focus groups. In reality, the sample characteristics resemble the African American demographic distribution in Louisville. For instance, given that the population of African American, non-heterosexual individuals in Louisville is small compared to heterosexual-identifying individuals, it is fair to expect that the sample would appear skewed towards heterosexual individuals. To improve recruitment of sexual and gender minority groups, a recruiter matching the characteristics of the target demographic was hired to specifically reach MSM and LGBTQ+ individuals who are considered a hidden and hard-to-reach population. Additionally, with the recognition that sexual and gender minority populations are highly marginalized and thus difficult to reach, this study utilized an incentivized method of sampling, respondent driving sampling (RDS), that facilitates the recruitment of hard-to-reach/hidden populations. With respect to this technique, nonrandom selection of initial seeds may potentially influence the sample characteristics of 
later recruits, hence more heterosexual participants in the sample. Moreover, for the purposes of the AFYA study, the data source for this analysis, commonality of risk across the populations, rather than unique differences in characteristics of each risk category, was of greater interest, to create a tailored PrEP promotion campaign to address low PrEP awareness among young African Americans across multiple risk categories. Finally, none of the participants screened into the AFYA study identified as a person who injects drugs (PWIDs). This may be due to the fear of being penalized upon disclosure, especially given the pervasive criminalizing drug polices and the war on drugs that disproportionately targets African Americans (Kerr \& Jackson, 2016). The lack of participants identifying as PWIDs could also be due to the fact that the current opioid crises in Kentucky does not reflect a prevalence among African Americans.

\section{Part Two of the Dissertation Study \\ Study Design}

Part two of the proposed study design utilized a grounded theory approach to address the second and third research questions (R2 and R3) by engaging ASOs who have been successful with PrEP initiatives (PrEP outreach/delivery) in the research process to qualitatively assess best practices for conducting PrEP outreach/delivery with African American priority groups. The intent was to utilize these findings, along with study findings compiled from part one, to provide recommendations to local ASOs for improving PrEP outreach and service delivery to African American priority groups.

Scaling up PrEP uptake is necessary to help decrease the HIV disparity gaps experienced by African American high-risk groups.

\section{Study Setting}


Phone interviews were conducted with key informants from ASOs outside of Kentucky, who have been successful in PrEP delivery among various risk populations, especially with African American high-risk groups. Phone call interviews were more convenient and cost-efficient, rather than traveling to conduct face-to-face interviews with the ASOs. Louisville ASO representatives, on the other hand, were interviewed inperson due to the ease of travel to ASOs which were conveniently located in close proximity to the University of Louisville.

\section{Inclusion Criteria (National ASOs and Louisville ASOs)}

For this study, key informants from ASOs in cities/states across the U.S. with specific characteristics described below (Table 1) were selected to participate in the study. Preference was given to organizations who demonstrated success with PrEP outreach services to African Americans. Successful ASOs had established PrEP-focused initiatives and demonstrated a) sustainability, b) robust client uptake, c) established procedures and protocols, and d) effective PrEP education outreach activities.

Table 1 National ASO Sample Study Eligibility criteria

\begin{tabular}{|cc|}
\hline National ASO Sample Study Eligibility criteria \\
\hline ASO Inclusion Criteria & Exclusion Criteria \\
\hline $\begin{array}{l}\text { Have an established and robust } \\
\text { infrastructure for PrEP service } \\
\text { delivery and outreach }\end{array}$ & $\begin{array}{l}\text { Does not have an established } \\
\text { and robust infrastructure for } \\
\text { service delivery and outreach }\end{array}$ \\
- $\begin{array}{l}\text { Have a proven track record of } \\
\text { establishing and implementing } \\
\text { successful PrEP-focused } \\
\text { initiatives, particularly those } \\
\text { that have done this with African } \\
\text { American communities }\end{array}$ & $\begin{array}{l}\text { Does not have an established } \\
\text { track record of PrEP outreach to } \\
\text { priority groups }\end{array}$ \\
\end{tabular}


- Currently engage in HIV service delivery, specifically, PrEP outreach to the various African American high-risk study target groups

- Are involved in PrEP activities for at least 1 year

- Demonstrate client uptake

- Are engaged in PrEP education
- Does not have established PrEP-focused initiatives and does not demonstrate a) sustainability b) robust client uptake c) established procedures and protocols d) effective PrEP education outreach activities

- Is not involved in PrEP activities for at least 1 year

- Does not demonstrate client uptake

- Is not engaged in PrEP education

For ASOs in Louisville, the inclusion criteria were (a) be an ASO (includes government entities like the health department and other clinic-based CBOs providing HIV service) located in Louisville metro area and (b) offer HIV services and PrEP delivery services, especially outreach to African American priority groups. Louisville ASOs were excluded if they did not offer PrEP services and did not engage in outreach to the community.

\section{Recruitment}

I conducted a rigorous internet search to find ASOs who currently participate in PrEP-delivery and have an established infrastructure for PrEP delivery, with emphasis on those who provide services to African Americans in the various high-risk groups. Once I identified potentially eligible ASOs in the internet search, I called the organizations to verify if they fit the criteria. I sent emails with the inclusion criteria and description of study intent (approved by IRB, see Appendix B) to ASOs who requested to have additional information about the study in writing. I also recruited ASOs in various 
locales upon recommendation from other ASOs. The internet search included CDCfunded CBOs whom the CDC considers lead CBOs in the HIV prevention partnerships and whom the CDC claims had demonstrated expertise and success in delivering effective HIV prevention strategies among populations with the greatest need. I intended to select ASOs working with priority populations most affected by the HIV epidemic, with a focus on those ASOs primarily working with African American groups to provide PrEP delivery and/or outreach. Emphasis was placed on cities such as California, New York and Atlanta, Georgia which have elevated HIV rates and have several communitybased organizations with established HIV prevention infrastructure, which includes PrEP delivery and outreach. Additionally, calls were made to various eligible ASOs to solicit participation in the study. Ten ASOs were eligible based on the inclusion criteria and agreed to the interviews. The selected representative from each of those organizations was contacted to determine the most appropriate individual to serve as a key informant for each of those organizations. The selected key informant then participated in the interview. The identified individual was contacted directly via the preferred method of communication (email or phone) to schedule a phone call interview. Incentives were not provided for this portion of the study. This process (from initial recruitment to completion of theoretical interviews took about six months (from September 2019 to March 2020). The average time between initially contacting an ASO to scheduling with a selected representative and completing the interview was 4 weeks. Times ranged broadly from two days to 4 months (both extremes of response time were outliers). 


\section{Sampling}

For one-on-one, in-depth, key informant interviews, I utilized expert sampling (a type of purposive sampling). "Expert sampling calls for experts in a particular field to be the subjects of the purposive sampling" (Etikan, 2016, p. 3). A convenience sample of ten ASOs outside of Louisville, KY was selected based on the established inclusion criteria for the national sample. The selected ASOs were asked to recommend an experienced staff member, who is knowledgeable about the organization's involvement with PrEP delivery and outreach to populations of interest, to participate in the interviews. Cleary, Horsfall, \& Hayter (2014) state that, "informants are selected because of their personal experience or knowledge of the topic under study" (p.473).

While there is no consensus in the literature regarding what sample size is appropriate for any given study, grounded theory approach recommends using the concept of theoretical saturation to ascertain when to stop interviewing. This entails sampling purposefully until all concepts of the developing theory are satisfied (Charmaz, 2014). Theoretical saturation was reached with 16 interviews (10 first round/initial interviews and six second round/repeat interviews). Memoing, especially analytic memoing, and constant comparison of data throughout the data collection process helped to finalize categories and subthemes (Birks et al., 2008; Charmaz, 2014; Strauss \& Corbin, 1994).

For the Louisville ASOs, only about three ASOs are currently conducting PrEP initiatives and all three of those ASOs were reached and agreed to participate in the study. Also, this part of the study was intended only to describe the current state of PrEP outreach and delivery for Louisville ASOs. Thus, theoretical sampling was not necessary. 


\section{Data Collection Procedures}

\section{Semi-structured interviews}

The choice of which data collection methods to use in each qualitative study may stem from the strategy or approach chosen for that given study as well as amount of structure needed for that particular study (Merriam \& Tisdell, 2015; Salazar et al., 2015). For instance, grounded theory strategy typically employs in-depth interviews and observations (Salazar et al., 2015). Interviews may be conducted in one of three forms: unstructured, semi-structured, or structured (Gill, Stewart, Treasure, \& Chadwick, 2008; Merriam \& Tisdell, 2015; Salazar et al., 2015). They may involve individuals in a oneon-one fashion or may be conducted with three or more individuals in a group, which is known as a focus group (Salazar et al., 2015).

I chose to utilize a semi-structured approach to this data collection process because I needed to ask questions to explore a specific area of our research topic factors influencing ASO PrEP engagement and outreach with African American priority groups. Semi-structured interviews are appropriate when the researcher knows enough about the topic to ask relevant questions (Merriam \& Tisdell, 2015). Unlike unstructured interviews, which do not require a guide but are, rather, free-flowing allowing topics to emerge from informal conversations, semi-structured interviews utilize a guide with predetermined questions (typically open-ended questions) about the research topic for the purpose of defining areas to be explored (Gill et al., 2008; Salazar et al., 2015).

"Although there is more structure to this form of interview, the open-ended questions still allow the participant to elaborate and provide significant details on his or her experiences" (Salazar et al., 2015, p. 242). Besides providing structure and guidance to 
the interview process, the semi-structured approach allows room for the investigator to ask additional probing questions to elicit more information from the participants' responses (Gill et al., 2008). Probing is important as it "entails an effort on the part of the interviewer, either verbally or nonverbally, to elicit more details, to guide the dialogue, to iterate the meaning of something said by the interviewee, or to allow the interviewee to feel comfortable in preparing his or her responses" (Salazar et al., 2015, p. 241).

\section{Interview Guides}

The rationale to employ a semi-structured guide stemmed from the ability of this approach to focus or guide discussion when a research topic already exists and the researcher seeks to elicit additional information about a specific area of interest (Gill et al., 2008). A semi-structured guide stirs the participants in the direction of the preset topic; however, the process is not fixed, and questions may be modified and probes (or further questions generated from initial questions) can be inserted to elicit deeper responses to enhance richness of the data (Rich \& Ginsburg, 1999; Salazar et al., 2015). Interviews were determined to be the best method of data collection for this portion of the study, for several reasons. Since the participants (ASOs) are located outside KY (in various parts of the country), direct observation was not possible. "Interviewing is necessary when we cannot observe behavior, feelings, or how people interpret the world around them" (Merriam \& Tisdell, 2015, p. 88). Also, since distance was a factor here, interviews were the most cost-efficient way to acquire data in this portion of the study. Additionally, interviews provide the a more naturalistic setting for data collection (than surveys) and allow for elicitation of unique responses from each participant (Rich \& 
Ginsburg, 1999). Representatives from various ASOs had the opportunity to provide unique responses about their experiences regarding PrEP delivery.

The development of the semi-structured interview guide (Appendix C), for the indepth interviews with key informants from ASOs outside Kentucky, was informed by focus group findings with target populations as well as information in the literature from previous research. A similar interview guide (Appendix D) was administered to Louisville ASOs to assess current PrEP outreach/delivery practices and strategies and to determine areas of improvement as foundation for tailoring recommendations. Both interview guides were reviewed by methods experts who are members of the AFYA team and pilot tested. This served to improve wording, particularly understanding questions and accuracy in capturing desired information. The documents were refined and utilized for subsequent data collection.

\section{Participant Consent}

Before commencing any aspects of data collection, a preamble consent (Appendix E) was administered to all study participants. Interviews were audio recorded with the permission of the participants. First round interviews ranged from 29 minutes to approximately 70 minutes. Second round interviews ranged from 13 minutes to approximately 59 minutes. Interviews were not incentivized.

\section{Self (the researcher) as the instrument}

In qualitative research, researchers are instruments too and thus are expected to show integrity by being self-reflective and transparent about their inherent "biases, predispositions, and assumptions regarding research to be undertaken" (Merriam, 2009, p. 219). To increase trustworthiness of any study, it is recommended that researchers be 
honest and transparent about their background and past professional experiences which may influence the conduct and interpretation of study findings (Creswell, 2013; Merriam, 2009).

\section{Positionality and Reflexivity Statement}

I am an African American, cis-gendered, female immigrant, born and raised outside of the U.S. I have lived in this country for only slightly under two decades. I am aware that being of a different culture and upbringing, my experiences inform my worldview, which may differ from that of my research participants. Prior to beginning my career in public health, I studied chemistry and worked in the biochemical and microbiology industries for a combined four and a half years before transitioning into public health. It was my keen interest in understanding how to prevent the spread of infectious and sexually transmitted diseases among persons of African descent in the diaspora that led me to transition out of the natural sciences into health care. During the last nine years, I have been involved in HIV research and prevention outreach among Black/African American communities. While attending a Historically Black College, I received training in qualitative as well as quantitative research methodologies and spent much of my time as a student researcher working with Black/African American populations. I also worked as a health counselor in a predominantly African American community with high rates of HIV/STDs and Hepatitis C, providing counseling, screening, and outreach services. As a "foreigner" with an extensive educational background and one who is part of a system that is already mistrusted by the African American community, I needed to gain the trust of the community. I began familiarizing myself with the African American culture and history, especially the unethical 
experiences African Americans endured with the medical and research community that have brewed mistrust of the system. Moreover, I became trained and certified to provide tailored HIV prevention instruction to Black/African American audiences within the context of their culture. I have contributed to the development of HIV prevention strategies for preventing HIV among minority and underserved populations and contributed to developing a needs assessment that informed the establishment of a PrEP clinic in Louisville, Kentucky. This vested interest in preventing HIV among this population is what informed my involvement in this current research project.

Thus, as a human instrument in the study, I must be conscious and aware that my background and experiences will undoubtedly inform the way that I interact with the participants and the data. In qualitative research, "it is reasonable to expect that the researcher's beliefs, political stance, cultural background (gender, race, class, socioeconomic status, educational background) are important variables that may affect the research process. Just as the participants' experiences are framed in social-cultural contexts, so too are those of the researcher" (Bourke, 2014, p. 2). If is fair to assume that my previous knowledge of the subject matter may influence my interpretation of what I think the participants mean by their responses. I must, therefore, be mindful of my own biases and ensure that they do not unduly affect the participants' responses or my interpretation of their responses. Additionally, as a person highly interested in this topic and one with previous experience as a HIV disparities researcher, health counselor, and HIV tester within the African American community, I must be conscious that my prior extensive knowledge does not unintentionally introduce bias in the way that the data is collected and interpreted. To ensure that this is not the case, participant checking (at least 
in the case of the in-depth interviews) was conducted to ensure that my interpretation of findings was consistent with what participants meant by their responses. Checking with the participants to confirm that their thoughts are accurately represented in the researcher's interpretation of the results adds credibility to the study. Additionally, although difficult to achieve with perfection, especially during a phone interview where non-verbal communication is impossible, I tried to remain neutral to the extent possible during the data collection process. I needed to be present and engaged with the participants; thus, I tried to minimize value-laden responses so as not appear to take any specific position regarding any of the concepts during data collection.

\section{Transcription}

All interview recordings in the study $(\mathrm{N}=16$ for national ASOs and $\mathrm{N}=3$ for Louisville ASOs) were transcribed by an external transcribing service. After interview transcripts were returned, I first listened to all audio recordings to verify that the transcription matched the information in the recording verbatim. All identifiable information was redacted before data analysis commenced. Transcription of data and data analysis was iterative throughout the duration of the data collection process.

Ongoing or sequential data analysis aided in ascertaining when theoretical saturation was reached and when to cap the number of interviews (Guest, Bunce, \& Johnson, 2006; Trotter II, 2012). This was determined with 16 ( $\mathrm{N}=10$ first round and $\mathrm{N}=6$ second round) interviews.

\section{Data Management}

Identifiable information (names, phone numbers, addresses) was removed from any study data collection materials to protect the identities of the participants. Each 
interview was assigned a unique identification (ID), written on the interview forms, interviewer notes, and transcripts. All data/documentation (interview forms, recordings, and field notes) gathered during field work were stored in a secure location and was not be shared with anyone who was not part of the research team.

\section{Data Analysis Strategy}

Constructivist grounded theory principles (line-by-line coding, focused coding, theory building and memo writing) were employed for this analysis (Figure 10) and aided in data synthesis to allow themes and theories to emerge from the data (Charmaz, 2014). A process of constant comparison whereby data is compared against data to refine meaning was also utilized as part of the analysis process as stipulated by CGT (Charmaz, 2014). Initial coding is the first step in a systematic data analysis process and entails making sense of the data by going through the transcripts line-by-line. This process makes the researcher open to the data, thus providing the opportunity to notice nuances contained within the data; the process also helps with identifying implicit concerns and explicit details in the data (Charmaz, 2014). Part of the data (N=7) was initially coded line-by-line using gerunds ("ing” words denoting actions). Initial codes were further refined to make focused codes for focusing the analysis (Charmaz, 2014). The handclustered codes $(\mathrm{N}=24)$ were further refined into the final codebook $(\mathrm{N}=18$ codes with definitions) using the most frequent and significant focused codes (Charmaz, 2014). The codebook was uploaded into Dedoose, a web-based data analysis software that organizes and facilitates coding (Dedoose.com). Myself and a colleague independently applied the final codebook to part of the data ( $\mathrm{N}=4$ and $\mathrm{N}=3$, respectively) to ensure consistency in code application and to increase credibility of the process. 
Figure 10 National ASO Key Informant Interviews Data Analysis process.

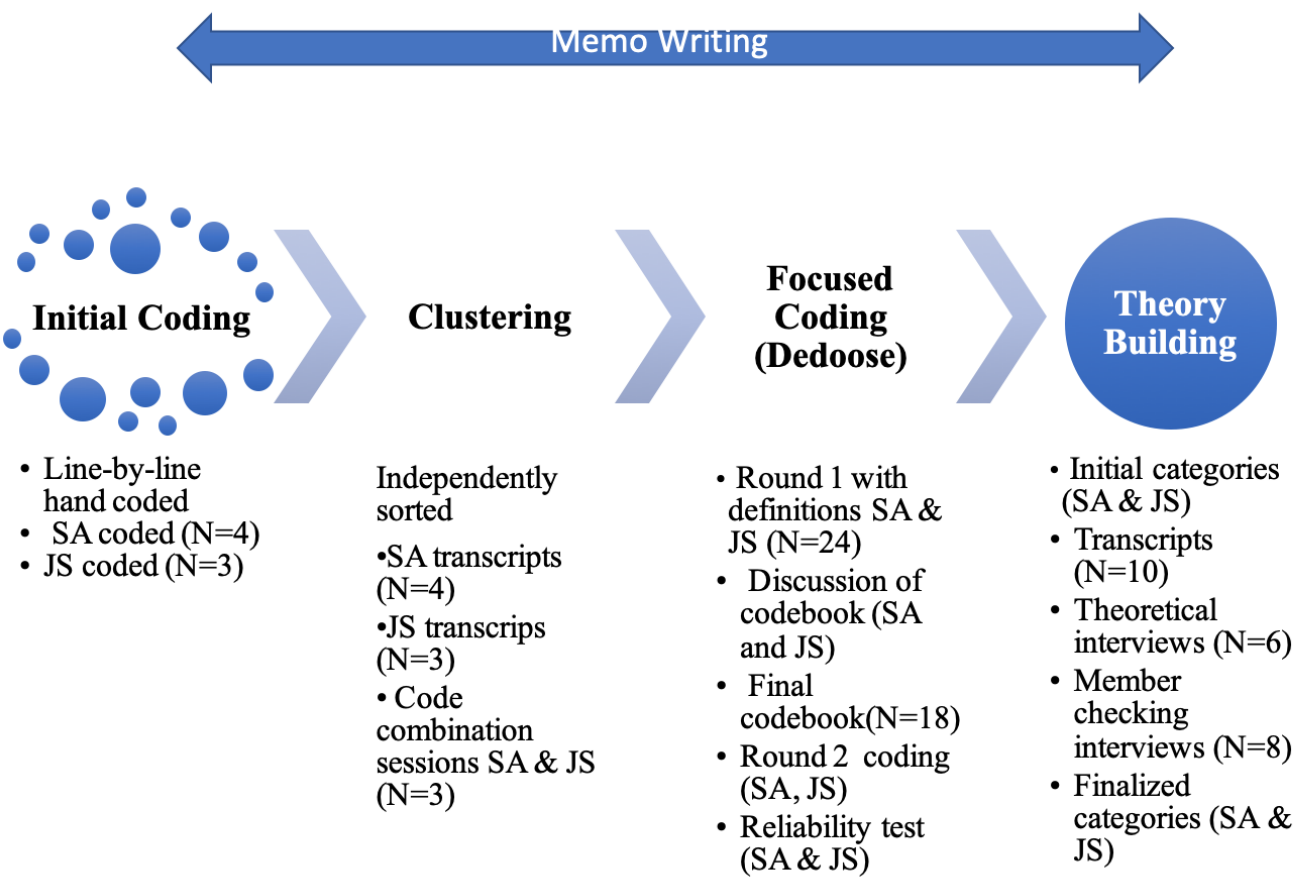

An inter-coder test (kappa test) (Cohen, 1960) was taken by the two coders. Interrater reliability is a statistical measure assigned between -1 and +1 , which determines the extent of agreement between two raters or coders (McHugh, 2012). This process typically involves two team members independently coding random excerpts from the transcripts, based on an existing, agreed upon, coding framework, to determine the percentage of agreement between the two coders. Reliability in this case is determined by an inter-rater consistency score, the kappa score. According to McHugh (2012), a score of $\leq 0$ indicates no agreement; $0.01-0.20$ indicates none to slight agreement; $0.21-0.40$ is fair agreement; $0.41-0.60$ is moderate agreement; $0.61-0.80$ is substantial agreement; and 0.81-1.00 is almost perfect agreement. Myself and a colleague independently coded four and three transcripts and took the kappa test. A pooled Kappa score of 0.92 indicated excellent inter-rater agreement among coders (Cohen, 1960) during the first attempt, but 
$100 \%$ agreement was desired. Thus, following a discussion among coders, a 100\% agreement was reached with the second Kappa test attempt. The final codebook was applied to code the rest of the data $(\mathrm{N}=10)$.

Memo writing was part of the entire process from data collection to theory building. This is important because "memo-writing provides a space to become actively engaged in your materials, to develop your ideas, and to fine-tune your subsequent datagathering" (Charmaz, 2006, p. 72). This process provides the platform for the researcher to become immersed in the data and to explore deeper meanings (Birks et al., 2008). This "interplay between researcher and data is crucial to the generation of knowledge that reflects the breadth and depth of human experience" (Birks et al., 2008, p. 69). While interacting with the data, the researcher makes observations and asks questions of the data before and during the coding phase and writes down side notes, which guide the analysis. Memo writing is also useful for defining and refining focused codes to be applied to the entire dataset for a more robust analysis (Charmaz, 2014). In this study, written memos were utilized during analysis to refine the coding process and to aid the process of generating themes from the data. Constant comparison of data also helped to finalize categories and subthemes (Charmaz, 2014; Strauss \& Corbin, 1994).

Computer software (Dedoose) was utilized to assist with data analysis. This software was only utilized for organizational purposes to manage the data. Data analysis in qualitative research is a daunting process and extremely time-consuming, especially when approached manually, hence the need for a computer program. "The computer has great capacity for organizing massive amounts of data, facilitating analysis and assisting communication with members of a research team" (Merriam, 2009, p. 195). Although the 
use of computer software does not necessarily make the analysis process less timeconsuming per se, it does greatly enhance the process of code formulation, application, and retrieval (Pope et al., 2000).

\section{Human Subjects Protection}

A study protocol was submitted to the University of Louisville Institutional Review Board (IRB) for review. This process was completed as part of the AFYA study with multiple amendments as needed (IRB number: 18.0020, Appendix F). The researcher and all research staff completed the required CITI training to ensure understanding of the rights of human subjects in research. Research staff also received training on procedures to protect participant identity and data during and after data collection. Preamble consent was read over the phone to all study participants who gave verbal consent indicating agreement to voluntarily participate in the study. The purpose of the study, any benefits (non-applicable), potential harm (very unlikely) to each participant, and the anonymity associated with study participation were clearly communicated to all participants. Participants were also informed of the intent to record the interview and focus group sessions, with their permission. In this study, no identifiable information was collected; numbers were assigned to interview participants. However, when identifiable information was revealed during the interviewing process, such information was removed and names redacted during the transcript cleaning process to protect the participants' anonymity. Participants were also informed of their ability to discontinue or withdraw from the study at any time without any consequences or punitive action taken against them. The potential risks associated with participation in this study 
were minimal and participants appeared to not experience discomfort with any questions in the study, despite the sensitive nature of the topic.

\section{Philosophical Assumptions and Interpretive Framework}

This study followed the methodological assumptions of qualitative inquiry, which were emphasized throughout the study. "The procedures of qualitative research, or its methodology, are characterized as inductive, emerging, and shaped by the researcher's experience in collecting and analyzing the data" (Creswell, 2013, p. 22). The study is based on tenets of constructivist grounded theory (Charmaz, 2014) and relies majorly on inductive reasoning for data analysis and reporting of findings. Grounded theory approach implores the researcher to allow a detailed understanding and explanation of the topic being studied to emerge from the data (Charmaz, 2006, 2014; Creswell, 2013; Glaser \& Strauss, 1967; Strauss \& Corbin, 1994). Its philosophy and methodology demand that the researcher respect the views of the participants and represent participants' perspectives without preconceived assumptions, while interpreting the data. That is, the researcher ought to see the world as the "research participants do-from the inside" (Charmaz, 2006, p. 14).

Furthermore, CGT is rooted in an interpretive constructivist worldview. This study was designed through the lens of an interpretive framework of social constructivism. Constructivists believe that multiple realities and interpretations exist and are socially constructed through the lived experiences of individuals and their interactions with others (Creswell, 2013; Merriam \& Tisdell, 2015; Salazar et al., 2015). In this worldview, meaning is co-constructed between the researcher and the research participant and ideas are emergent (Charmaz, 2014; Creswell, 2013); that is, individuals, 
researcher, and research participants alike, have varying interpretations of the studied world and create meanings through interaction and sharing of various viewpoints (Charmaz, 2014; Creswell, 2013). Additionally, social constructivism assumes that interpretations are subjective (context-specific) and may be shaped by cultural norms, social, and historical factors within the lives of the individual (researcher and participant alike) (Creswell, 2013). I was conscious of these assumptions during this study by first engaging in the process of member checking to ensure that participants' views were not misrepresented and, second, ensuring that all interpretations of findings were rooted in data as depicted by the display of quotes to support every claim or conclusion made by the researcher with respect to the study results.

\section{Criteria for Establishing Rigor and Trustworthiness}

Trustworthiness or authenticity of research ensures that the observations, interpretation, and conclusions of a study are accurate, acceptable, dependable, and trustworthy (Lincoln \& Guba, 1985; Merriam \& Tisdell, 2015). This is contingent upon rigor and various strategies employed at different stages of the research process (Creswell, 2013; Creswell \& Miller, 2000; Golafshani, 2003; Merriam, 2009; Patton, 1999; Seale, 1999). Several strategies have been recommended to ensure trustworthiness of research in qualitative research (Creswell, 2013; Merriam, 2009; Merriam \& Tisdell, 2015; Patton, 1999; Salazar et al., 2015). For this study, the criteria for trustworthiness by Lincoln and Guba (1985) that includes credibility, transferability, dependability, and confirmability is utilized to describe how rigor was achieved during the research process. 


\section{Credibility}

This is defined as plausibility or confidence in the truthfulness of the findings (Lincoln \& Guba, 1985; Tracy, 2010). Credibility can be accomplished by giving careful thought to the entirety of the research process from its conceptualization to data collection, interpretation, and reporting (Merriam \& Tisdell, 2015). Several strategies have been recommended to improve credibility in qualitative research (Creswell, 2013; Merriam, 2009; Patton, 1999; Salazar et al., 2015; Tracy, 2010). The following strategies were utilized in this study to ensure credibility: 1) triangulation, 2) member checks or respondent verification, 3) adequate engagement in data collection or spending extensive time in the field to build trust between researcher and participants, 4) researcher's position or reflexivity (clarification of biases or positioning one's self and being selfreflective as a researcher), and 5) peer examination or peer review and debriefing.

\section{Triangulation}

This entails using various sources of data to corroborate evidence (Lincoln \& Guba, 1985; Merriam \& Tisdell, 2015). This dissertation achieved data triangulation by utilizing multiple data sources: focus groups, key informant interviews with Louisville ASOs, and national sample of ASOs. Triangulation is one of the most known and typically utilized strategy for ensuring trustworthiness of study findings (Golafshani, 2003; Merriam, 2009; Merriam \& Tisdell, 2015; Seale, 1999). The process of triangulation not only ensures that weaknesses of some approaches get counterbalanced by the strengths of other approaches, but it also enables a more complete understanding of the concept (in this case, reticence to PrEP engagement, outreach and uptake among 
African Americans); further, it allows for a more conclusive interpretation and conclusion (Salazar et al., 2015).

\section{Member checks or respondent verification}

This entails going back to some of the study participants to verify that the researcher's interpretation of findings and conclusions reflect participants' meaning; that is, "rings true" (Lincoln \& Guba, 1985; Merriam \& Tisdell, 2015). Member checking was done for the in-depth key informant interviews during subsequent/repeat interviews $(\mathrm{N}=8)$ with the national sample of ASOs to confirm emerging theoretical categories and finalized process for the developed framework. For the focus groups, AFYA team members who were present and who participated in the data collection process reviewed the study findings for accuracy to ensure that interpretations were closely representative of what was said by the participants, since the AFYA study could not go back to the focus group participants to verify the findings.

\section{Adequate engagement in data collection or spending extensive time in the}

\section{field}

Spending extensive time in the field builds trust between researcher and participants and ensures a deeper understanding of the culture and perspectives of the participants on the subject or topic under study (Lincoln \& Guba, 1985; Merriam \& Tisdell, 2015). With respect to this, the AFYA research team made effort to be intentionally present within the community during the recruitment process at venues frequented by priority groups. Through this means, our team became familiar with and built relationships with potential participants, many of whom became comfortable with relating with our team as well as referring their friends to participate in our study. For the 
interviews with key informants of ASOs, interview duration (lasting approximately 45 minutes) as well as consistent email and phone correspondence throughout the recruitment, data collection, and member-checking process ensured considerable time spent in the field with participants.

\section{Reflexivity}

It is recommended that researchers be honest and transparent about their background and past professional experiences that may influence the conduct and interpretation of study findings (Creswell, 2013; Merriam \& Tisdell, 2015). This was clearly articulated in earlier sections (see researcher as the instrument/positionality and reflexivity statement). Reflexive notes were maintained throughout the research process to articulate the researcher's position and perceptions of the process (Charmaz, 2014; Creswell, 2013).

\section{Peer examination or peer review or debriefing}

In this case, peers (research chair, committee, and another colleague) reviewed the study product to ensure that interpretations and conclusions are consistent with data (Creswell, 2013; Merriam \& Tisdell, 2015). Debriefing was ensured during focus groups as at least two persons from the AFYA study team were present during focus group data collection and debriefed afterwards. This enables comparison of notes during review and debriefing sessions to ensure accurate reporting or representation of data collection sessions.

\section{Transferability}

This refers to the ability of others to look at a study and be able to extrapolate it and apply it to other similar situations; it is made possible through rich, thick descriptions 
of study procedures (Lincoln \& Guba, 1985; Merriam \& Tisdell, 2015). Merriam \& Tisdell (2015, p. 257) assert that " toaday when rich, thick description is used as a strategy to enable transferability, it refers to a description of the setting and participants of the study as well as a detailed description of the findings with adequate evidence presented in the form of quotes from participant interviews, field notes and documents." I ensured rich, thick description of the study procedures that allows others to be able to determine transferability of study findings to other locations or scenarios. Thus, I expect that the studies in this dissertation can be extrapolated and replicated by persons who find them helpful and thus studies can be applied to populations who are similar to those in this study, namely African American priority high-risk groups and ASOs.

\section{Dependability}

Dependability is achieved using an audit trail (a detailed description of the research process) and is referred to as consistency in interpretation of findings with respect to the data (Lincoln \& Guba, 1985; Merriam \& Tisdell, 2015). This is achieved through a detailed description of the entire research processes as shown in the methods section of this dissertation.

\section{Confirmability}

This refers to the extent to which study findings are shaped by the views of the participants rather than the researcher's biases or interests or motives and is achievable

through audit trails, triangulation, confirmability trail, and reflexivity (Lincoln \& Guba, 1985). These tenets of confirmability have been demonstrated (described above) to ensure that the final interpretation of findings closely reflect participant views. 


\section{Study Limitations}

This part of the study utilized purposive sampling for key informant interviews. Although purposive sampling is a convenience sampling approach, it is advantageous for selecting persons who are experts on the topic being researched. Purposive sampling may be prone to researcher bias, which stems from subjective selection of study participants. However, this limitation was taken into consideration by a clearly defined inclusion and exclusion criteria as well as participant selection process informed by research purpose and questions. Salazar et al. (2015) asserts that participant selection for a study should be based on the purpose of the research study which relates to the research questions. Rather than thinking about generalizability, based on statistical probability, as is the case in quantitative approaches, qualitative researchers recommend the concept of extrapolation (Merriam, 2009). Thus, generalizability is not a limitation of qualitative research.

Despite low response rate from potential ASO key informants (most likely due to heavy workloads and busy schedules), the sample was still determined to be adequate for the study analysis as theoretical saturation was reached using a theoretical sample $(\mathrm{N}=6)$ in addition to the original sample $(\mathrm{N}=10)$ achieved through repeat interviews with some of ten key informants from the initial sample. It took a long time (about 4 weeks to even 4 months in one case) before several ASO representatives could finally schedule a time to complete the interview. Several more ASOs would have participated, but finding the time to commit to a phone interview was cited by some as reasons for their slow response time. In the end, sixteen interviews were determined sufficient for the final conclusions drawn from the data analysis, as theoretical saturation was reached. Moreover, sample adequacy, rather than sample size, provides better justification for theoretical saturation (Hennink, Kaiser, \& Marconi, 2017). Also, the length of the interview time (an average 
of 45 minutes) spent with each participant, helped to elicit thick and nuanced data for a rich analysis and to ensure rigor and trustworthiness of study findings (Creswell, 2013; Lincoln \& Guba, 1985).

\section{Dissemination of Study Findings}

The strategies for disseminating study findings to ASOs in Louisville may include a) distribution of recommendation packet to organization representatives and b) a knowledge translation activity is expected to be executed upon availability of funds. 


\section{CHAPTER IV: PAPER I}

\section{UNDERSTANDING INTRA-PERSONAL FACTORS IMPACTING PRE- EXPOSURE PROPHYLAXIS (PREP) ENGAGEMENT AMONG YOUNG AFRICAN AMERICANS}

\section{Introduction}

In the previous chapter, I described the methods employed to accomplish the purpose of this dissertation. I also described and provided justification for the approaches utilized to answer the various research questions. To reiterate, the purpose of the dissertation study was three fold: (1) to qualitatively explore and understand the barriers and facilitators to PrEP uptake among various African American youth (18-29 years old) around Louisville, Kentucky in priority groups: persons who are at high-risk for HIV; (2) to examine effective strategies for scaling up PrEP engagement and outreach among African American priority groups; and (3) to develop recommendations for local AIDS service organizations (ASOs) to improve PrEP outreach/delivery efforts among African American priority groups.

This chapter provides partial evidence derived from focus groups with African American youth groups in Louisville, KY who are at high risk for HIV. This evidence satisfies three things: (1) it partially addresses the first part of the dissertation's purpose; (2) it answers the first research question: What are the multi-level barriers and facilitators to PrEP engagement, from the perspectives of African American youth groups in 
Louisville, KY who are at high risk for HIV?; and (3) it fulfills study aim one: To explore and understand barriers and facilitators to, and engagement with, PrEP uptake among African American priority groups. This chapter specifically addresses individual level/intrapersonal facilitators or barriers to PrEP engagement and uptake among young (18-29-year-old) African Americans residing in Louisville and who demonstrate heightened HIV vulnerability.

\section{Background}

Despite advances in HIV prevention and treatment in the U.S., racial, sexual, and gender minority groups continue to experience higher disease burden. African Americans are disproportionately affected by HIV. The CDC (2020) estimates 37,832 new HIV diagnoses in 2018. Although African Americans only represent $13 \%$ of the population, they accounted for the highest proportion (43\%) of those cases compared to any other racial group (Centers for Disease Control and Prevention, 2020a). This

disparity is more apparent across some subgroups within this population such as gay and bisexual men, women, and youth. For instance, rising annual HIV diagnosis has been observed among young African American gay and bisexual men, particularly those within the age group of 25-34 years old (Centers for Disease Control and Prevention, 2018a, 2020a). African American women are also heavily impacted as they account for $59 \%$ of HIV cases diagnosed among women (Centers for Disease Control and Prevention, 2019a). In fact, it is estimated that an African American woman is 17 times more likely to be diagnosed with HIV in her lifetime compared to a White woman (Hess et al., 2017). 
Given this disparity, highly effective strategies are warranted for curbing the epidemic and reducing the disease burden among African Americans. In response to this need, the CDC proposed high impact initiatives for prevention that include the use of biomedical interventions such as HIV Pre-exposure prophylaxis (PrEP) (Centers for Disease Control and Prevention, 2014b). Truvada for PrEP — a once daily, oral prescription medication — was approved by the FDA in 2012 as a major biomedical prevention tool for decreasing new infections in HIV-negative individuals at heightened risk (US Food and Drug Administration, 2012). In 2014, the CDC recommended PrEP for use in persons at substantial risk for HIV. This includes serodiscordant couples, or those in an ongoing relationship with an HIV-positive partner only in situations where the HIV-positive partner is neither taking antiretroviral medications nor has a suppressed viral load. This group also includes heterosexual men and women who do not regularly use condoms during sex with partners of unknown HIV status and who are at substantial risk such as injection drug users. Finally, gay or bisexual men who have anal sex without a condom are also at substantial risk for HIV (Centers for Disease Control and Prevention, 2014b). The FDA also approved Descovy, another PrEP medication, in 2019. However, indications for use did not include cisgender women since at the time of the approval, the effectiveness of Descovy had not been evaluated on persons at risk for HIV through receptive vaginal sex (U.S. Food and Drug Administration, 2019).

The efficacy of PrEP in decreasing the number of new HIV cases in various priority groups has been demonstrated by numerous clinical trials with an efficacy rate of more than 92\% observed in some cases (Baeten et al., 2012; Choopanya et al., 2013; Grant et al., 2010; McCormack, Dunn, Desai, Dolling, Gafos, Gilson, Sullivan, Clarke, 
Reeves, Schembri, et al., 2016). Despite this effectiveness, the uptake of PrEP has been marginal among priority groups like high-risk African Americans who stand to benefit the most from the intervention (Buchbinder \& Liu, 2018; Eaton, Driffin, Bauermeister, et al., 2015; Eaton, Matthews, et al., 2017). For example, various studies reporting on national trends in PrEP prescription access and use show that fewer African Americans have access to PrEP and fill fewer PrEP prescriptions (Bush, Ng, Magnuson, Piontkowsky, \& Mera Giler, 2015; Siegler, Bratcher, et al., 2018).

Although PrEP uptake was reported to have increased about 500\% between 20132015, uptake was considerably low among African Americans: 75\% PrEP prescriptions were filled by Whites, compared to only $10 \%$ by African Americans (Bush et al., 2015). Other studies also show a low proportion of African Americans were screened for PrEP services relative to the HIV diagnoses among them (Elopre et al., 2017). A more recent study examining national quarterly rates of PrEP prescriptions revealed that only 5\% of the 1.2 million persons indicated for PrEP are receiving PrEP protection (Siegler, Mouhanna, et al., 2018). This report further showed that by the end of the second quarter of 2017, the national PrEP prevalence was 23/100,000. The PrEP-to-need ratio (PrEP prescriptions relative to HIV diagnosis) was only $1.5 / 100,000$. Moreover, states with the highest number of African Americans had the lowest PrEP prevalence as well as PrEP-toneed ratio, an indication that very few African Americans are accessing PrEP in states where it is needed the most.

These stark disparities observed in HIV burden as well as PrEP uptake underscore the importance of developing a deeper understanding of factors precluding PrEP engagement among African American groups demonstrating heightened HIV vulnerability, such as 
those practicing or who have partners practicing high-risk behaviors. As such, this study aimed to gain a deeper understanding of intrapersonal factors such as perceptions, attitudes, and beliefs that influence engagement with and uptake of PrEP among young African Americans in a southern U.S urban city. This aim indicated that a qualitative approach was suitable wherein we conducted focus groups with various young African American groups demonstrating heightened HIV vulnerability.

We utilized constructs from Theory of Reasoned Action/Theory of Planned Behavior (TRA/TPB; Figure 11) as theoretical sensitizing concepts to elicit a broad range of characteristics shaping African American high-risk groups' intention to use PrEP for HIV prevention. TRA/TPB posits that attitudes towards a behavior (PrEP use), subjective norms, and perceived behavioral control factors predict the performance of a behavior (PrEP use) or more precisely influence the intention to perform that behavior (Ajzen, 1991). As such, these factors were seen as important for elucidating reasons behind low PrEP uptake among this population and subsequently informing multi-media PrEP campaigns to increase PrEP awareness among African American youth.

\section{Methods}

\section{Rationale for study population}

The data for this study was part of the AFYA PrEP study, which was conducted in Louisville, Kentucky. Kentucky is a fitting site for this inquiry as the state's HIV surveillance data shows that between 2011 and 2015, African American women were 9.6 to 12.8 times more likely to be diagnosed with HIV compared to White women, and African American men were 4.4 to 6.2 times more likely to be diagnosed with HIV compared to White men (Kentucky Cabinet for Health and Family Services Department 
for Public Health HIV/AIDS Branch, 2017). Moreover, Kentucky surveillance reports have consistently indicated a higher annual incidence of HIV among African American youth, especially youth between the ages of 20-29 years old, regardless of gender (Kentucky Cabinet for Health and Family Services Department for Public Health HIV/AIDS Branch, 2014, 2016, 2017).

\section{Design}

We conducted a qualitative study (Merriam \& Tisdell, 2015) using constructs within TRA/TPB (Figure 8) as theoretical sensitizing concepts. Theory building was not a goal for this study, but it was informed by constructivist grounded theory (CGT) (Charmaz, 2014) that allows for the use of sensitizing concepts and has its philosophical underpinnings in symbolic interactionism (SI) (Blumer, 1986) and pragmatism (Lewis, 1976) (both of which focus on meanings and actions). SI posits that human beings act towards a situation (PrEP use) based on symbolic meanings (interpretations) and human interactions, informed by the development of the focus group guide.

For theoretical sensitizing concepts, we specifically drew upon two constructs from the TRA/TPB: attitudes and perceived behavioral control factors to explore how intrapersonal characteristics of African American youth at high risk of HIV may inform their decision to either engage or not engage with or use PrEP for HIV prevention. Based on TRA/TPB, we expected that if individuals have less negative perceptions, positive attitudes towards PrEP use, and high perceived behavioral controls (e.g. are aware of PrEP, have PrEP knowledge, have confidence to adhere to taking PrEP), they will be more willing to engage with and use PrEP. 
Figure 8 Adapted conceptual framework of TRA/TPB.

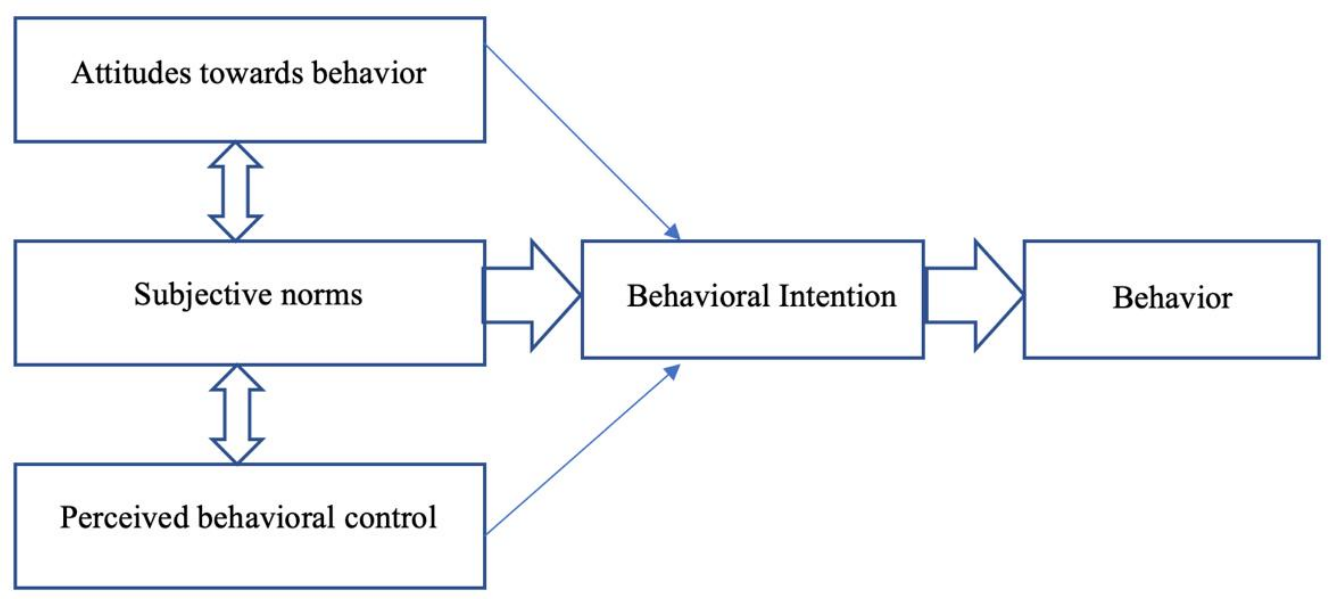

Source: Theory of Planned Behavior (Ajzen, 1991).

Using constructs from TRA/TPB as sensitizing concepts, we developed a semistructured focus group guide with various topics (Appendix A). This was constructed and reviewed across multiple iterations through a collaborative and multi-disciplinary team. This included experts in HIV and qualitative field research from social work, public health promotion, behavioral sciences, and medicine. The guide was pilot tested before being utilized to collect data. Focus groups were conducted at university facilities conveniently accessible to participants. Sample questions from the focus group guide are shown in Table 2.

Table 2 Sample Focus Group Questions

\section{Sample focus group questions}

- Have you ever heard of PrEP (pre-exposure prophylaxis), a daily medication that can prevent people from becoming HIV positive? family, social media, television, etc.?

- What do you think about PrEP?

- Would you consider getting PrEP yourself? Why or why not?

- Can you think of any reasons why young people would or would not want to get PrEP? 


\section{Participants/Sampling, Recruitment, and Enrollment}

Participants qualified to be in the study if they self-identified as an African

American, were between the ages of 18-29 years old, resided in the Louisville Metro area in Kentucky (specifically west Louisville), and self-reported heightened HIV vulnerability fitting the description of at least one of these high-risk groups: men who have sex with men (MSM), transgender persons, LGBTQ+, persons who inject drugs (PWIDs), and heterosexual persons exhibiting heightened HIV vulnerability (commercial sex workers, serodiscordant heterosexual couples, heterosexual persons who do not use condoms or have partners with unknown HIV status, relationships with sexual concurrency). It should be noted that the AFYA study focused on commonality of risk among participants in this case, rather than on risk differences among groups, although some differences were observed and reported across various priority groups. The original aim of the study was not to highlight distinct differences across each group per se.

Participants were recruited through: (i) flyers at local community-based organizations (CBOs) known to provide HIV prevention services to any of the target high-risk groups, a Syringe Exchange Program (SEP), grocery stores, a local university, the local health department, and community events; (ii) hired recruiters to help recruit the hard-to-reach groups (LGBTQ+); (iii) tabling at local churches, LGBTQ+ balls, gay pride parades, and parties; (iv) advertisements (print and social media); (v) attending community organizations and neighborhood association meetings; (vi) word-of-mouth to social networks; and (vii) by direct referral of peers through the method of respondent driven sampling (RDS). Much of the recruitment happened through RDS, during which 
an individual (a seed) was identified based on specific characteristics fitting the inclusion criteria. This individual was then asked to refer or nominate another individual with similar characteristics to participate in the study (Trotter II, 2012). Each seed who participated in the study received $\$ 35$ for participation, and could recruit up to three of their peers into the study and the seed received an additional incentive of $\$ 10$ for each peer who participated (Heckathorn, 1997).

\section{Data Collection Procedures}

We screened and enrolled a total of 63 participants into one of 11 focus groups, based on self-reported risk characteristics (Table 3). Team members that were trained and experienced in qualitative data collection conducted the focus groups. Most team members identified as African American. Team members administered informed consent to all study participants before focus group data collection commenced. Following informed consent, one team member facilitated, while the other team member took notes in all but two focus groups (where there was only the facilitator present). Focus groups lasted approximately 60 minutes and were audio recorded using a digital audio recorder. After each focus group session, team members debriefed and compared notes to ensure accurate reporting and representation of data for enhanced study credibility (Creswell, 2013). The University of Louisville Institutional Review Board reviewed and approved study procedures.

\section{Data Management and Analysis}

At the end of each focus group, the audio files were transferred from the recording device to a password-protected computer and saved on a secure university server. Transcripts were checked for accuracy by listening to audio recordings to ensure 
alignment with transcript verbiage. A total of 11 focus groups $(\mathrm{N}=63)$ were transcribed verbatim by two AFYA study team members and a transcription service (Rev.com). Identifying information was redacted from transcripts before analysis commenced using Dedoose qualitative analysis software (Dedoose.com). Constructivist grounded theory (CGT) analytic techniques (initial and focused coding) (Charmaz, 2014) and thematic coding (Strauss \& Corbin., 2015) were utilized for a rigorous and systematic data analysis illustrated in Figure 9. Since, theory development was not the goal of this study, we only utilize CGT coding techniques to elicit participants' lived experiences relating to PrEP use and engagement. Memoing and constant comparison of data helped to finalize categories and subthemes (Birks et al., 2008; Charmaz, 2014; Strauss \& Corbin, 1994) to inductively generate themes (Merriam \& Tisdell, 2015; Strauss \& Corbin., 2015) to describe factors impacting participants' experiences with PrEP.

Initial codes were derived from line-by-line coding of four out of 11 transcripts during the initial coding process that allowed codes to emerge from the original data. These codes were combined and grouped into focused codes that were clearly defined to highlight the underlying properties of each code. Two members of the team worked independently and together to refine codes and negotiate code definitions to arrive at a finalized version of the codebook (with focused codes). Some sub-codes were derived to help create subcategories within the main focused codes in order to provide an in-depth analysis of the data. 
Table 3 Focus Group Sample Description.

\begin{tabular}{|l|l|l|l|}
\hline Group & Group Composition & $\begin{array}{l}\text { Focus } \\
\text { Group ID }\end{array}$ & $\begin{array}{l}\text { Number of } \\
\text { Participants }\end{array}$ \\
\hline 1 & MSM-only & MS1 & 3 \\
\hline 2 & MSM \& heterosexual male mixed group & MS2 & 10 \\
\hline 3 & LGBTQ+ only Group & LG1 & 4 \\
\hline 4 & LGBTQ+ \& heterosexual male mixed group & LG2 & 5 \\
\hline 5 & Heterosexual male- only & HM & 3 \\
\hline 6 & Heterosexual female-only group 1 & HF1 & 7 \\
\hline 7 & Heterosexual female only group 2 & HF2 & 4 \\
\hline 8 & Heterosexual mixed-gender group 1 & HMG1 & 4 \\
\hline 9 & Heterosexual mixed-gender group 2 & HMG2 & 7 \\
\hline 10 & Heterosexual mixed-gender group 3 & HMG3 & 9 \\
\hline 11 & Heterosexual mixed-gender group 4 & HMG4 & 7 \\
\hline N & Total Number of Participants & & 63 \\
\hline
\end{tabular}

Themes were further derived from the second round of coding. Two team members independently applied the final codebook to the entire dataset to ensure consistency in code application and to increase credibility of the process. A pooled kappa score of 0.90 indicated excellent inter-rater agreement among coders (Cohen, 1960). The team discussed and approved the final coding. One team member (SA) looked over final coded data to ensure the accurate representation of data. The team discussed the finale themes. We utilized a process of inductive reasoning during the entire coding process to allow final themes to evolve (Charmaz, 2014). Constructs from TRA/TPB only served as sensitizing concepts to elicit broad, rich, and nuanced data to enable a deeper understanding of why PrEP engagement is low among the target population. That is to identify the underlying factors that influence intention to engage or not engage with or use PrEP for HIV prevention. Therefore, themes were not deductively derived to fit within each of the individual constructs of these theories. Also, findings relating to norms and behavioral controls from this study are reported elsewhere. 


\section{Results}

All focus group participants $(\mathrm{N}=63)$ were African Americans between the ages of 18-29 years old and residing in Louisville, KY. For the purposes of this current analysis and corresponding results, focus groups broadly comprised of a) MSM only (n=3); b) heterosexual male only $(\mathrm{N}=3)$; c) heterosexual female only $(\mathrm{N}=11)$; d) LGBTQ+ (sexual and gender minority) only ( $\mathrm{N}=4)$; e) two groups of MSM and LGBTQ+, each mixed with other heterosexual males $(\mathrm{N}=15)$; and $\mathrm{f})$ mixed heterosexual males and females $(\mathrm{N}=28)$. Comparisons were made across broad groups to highlight significant differences observed across risk categories.

\section{Common derived themes from data}

We identified several themes and grouped them into four main categories described below. The final categories included (1) PrEP awareness/knowledge (2) perceived HIV risk and PrEP need, (3) fears and reservations about PrEP, and (4) acceptability of PrEP. The relationships among these four categories are depicted by Figure 11. Themes within the four categories influenced intention to use PrEP (willingness/unwillingness to use PrEP) either positively or negatively. Some of the categories had multiple sub-categories. Fears and reservations about PrEP had three subcategories: (a) misperceptions about PrEP eligibility, (b) fear of side effects and drug interactions, and (c) adherence beliefs (or stance on adherence). Acceptability of PrEP had two sub-categories: (a) acceptability of PrEP as a viable HIV prevention approach and (b) acceptability of PrEP in its current pill form.

Findings revealed that PrEP awareness/knowledge influenced perceived HIV risk and need for PrEP. Additionally, awareness/knowledge, along with fears and reservations, influenced acceptability of PrEP. These factors influenced intentions for 
PrEP use and engagement. That is, these factors either acted as facilitators or barriers to PrEP use and engagement. Higher PrEP awareness/knowledge, positive perceptions and attitudes served as facilitators towards PrEP use and engagement, while low to no PrEP awareness/knowledge, negative perceptions, and attitudes, on the other hand, served as barriers towards PrEP use and engagement.

Figure 11 Relationship Among Themes: Intrapersonal Factors Influencing Intention to Use PrEP.

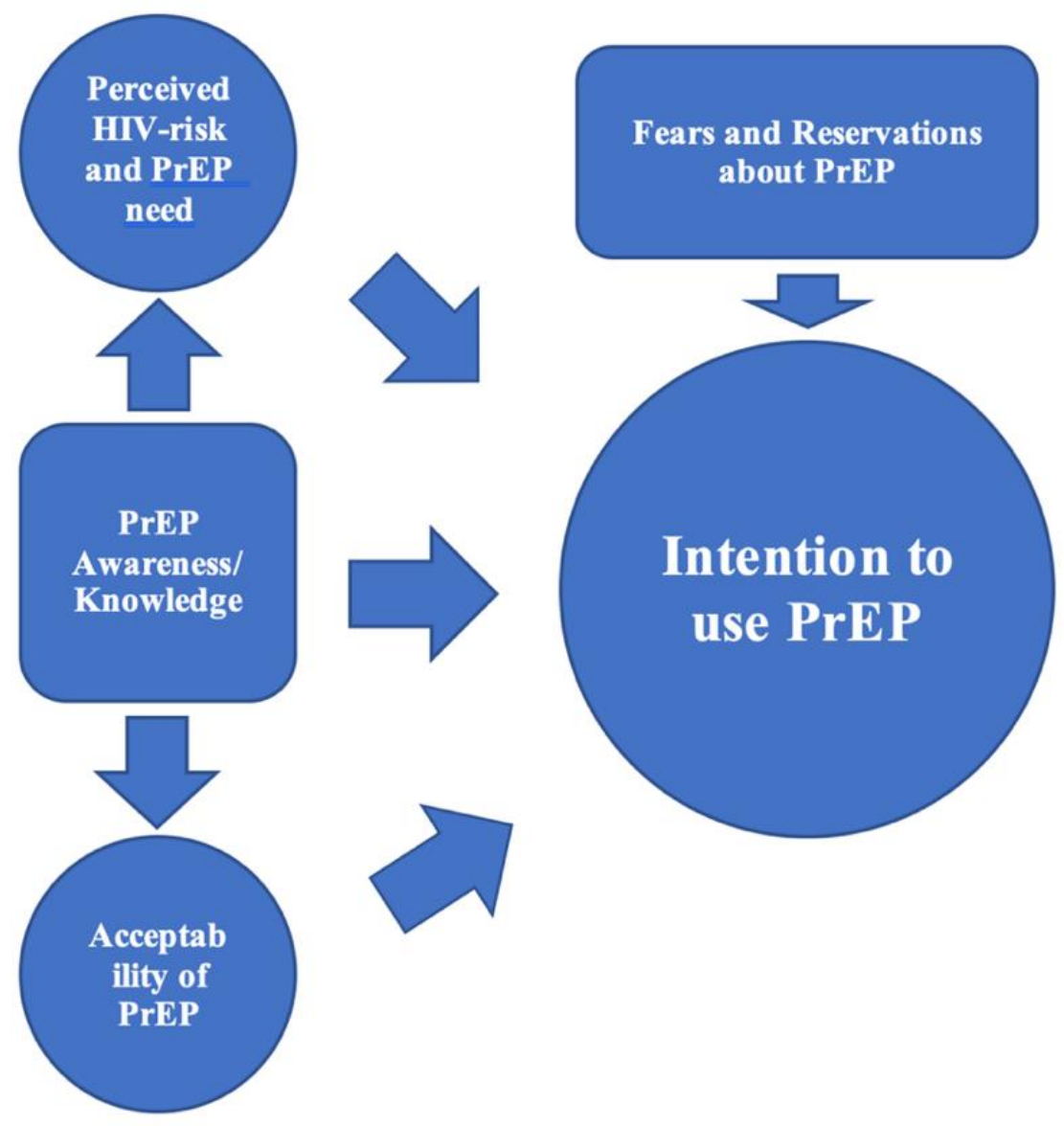

\section{PrEP awareness/knowledge}

Most of the participants across all 11 focus groups indicated that they had never heard about PrEP prior to the study. When the focus group facilitators posed the question, "Have you ever heard about HIV PrEP, Pre-exposure prophylaxis?", many 
participants, across all priority groups, responded by simply saying, "No." Others responded by saying that they had never heard about it. Some of the participants acknowledged that hearing about PrEP in the study was their very first introduction to PrEP. They said such things as,

"Not before this [study]." - Participant, heterosexual men-only group

"I seen the paper [study survey] yesterday. It was my first time hearing about it."

— Participant, heterosexual mixed (male and female group)

“... I haven't heard of it before yesterday.” — Participant, heterosexual mixed

(male and female group)

Heterosexual participants, especially, demonstrated lower levels of PrEP awareness and PrEP knowledge compared to their sexual and gender-minority counterparts (LGBTQ+ and MSM- identifying groups) in the study. In one of the female-only groups, one person said:

“... And there's only two people in here, or three, that has heard about it. So... it's not out there." - Participan,t heterosexual female-only group

Not only were most participants in the study unaware of PrEP, but many were not knowledgeable about it. This lack of knowledge was apparent in some of the participants' questions and comments such as:

"I don't know nothing about it." — Participant, MSM \& heterosexual male mixed group.

"I'm still kind of like, is it to prevent HIV or is it to, like, regulate you while you have HIV? - Participant, heterosexual female-only group 
"I got a question. It's about PrEP. So, you don't have to have AIDS to take it?"

- Participant, heterosexual mixed group

Thus, while many were unaware of PrEP, even fewer participants understood the purpose of PrEP or who is indicated to use PrEP. A small segment of participants, however, mostly from the LGBTQ+ groups, were aware and demonstrated some knowledge of PrEP. These participants heard about it on a TV commercial, social media, or from a friend or family member who had previously used PrEP and told the participant about it. Some participants from the LGBTQ+ groups noted:

"I heard it from a friend, television, I heard it through I think ... A commercial too.”- Participant, MSM mixed group

"They was all talking about it, my little gay friend he was talking about it, he was gonna take some. He talked about taking some." — Participant, MSM mixed group

"When I stayed in [city], whatever, I was talking to someone that was HIV positive and he was going to my house and they introduced me to it, but I never got it. I never got signed up for, well I signed up for it, but never got any, you know what I'm saying. I never accepted...” — Participant, MSM-only group "Yeah, I've heard about it for a few years. For a while just because it would be on social media, particularly Grindr. There was folks taking it a lot, but there was a lot of hesitancy. And I know it reduces your chances of like 92 to $98 \%$." Participant, MSM mixed group 
In addition to these participants, very few from heterosexual female-only groups also indicated exposure to PrEP through a commercial, on the radio, and from a family member.

"I think I've seen the commercials for it now."

"I heard about it on the radio, but pretty much what you just said...You can use it to prevent yourself from getting it [HIV]."

"It's funny 'cause my mom told me about it first, and she did not know about it." Overall, participants expressed various reasons why they believed they had not heard about PrEP prior to the study. Some felt that location was a factor. They assumed, for instance, that a person was more likely to hear about PrEP in spaces with more LGBTQ+ individuals. One participant remarked:

"I feel like it depends on you because it depends on what spaces you feel comfortable going into. So, like maybe if you're in a space where there's more transgender people you're gonna hear about it more but if you're in an area that's like a football or basketball game you probably won't hear about it as much. And then it depends on how educated they are because... I wasn't aware that it was also for straight people. So you also have to educate yourself about it."

Other participants felt that exposure to PrEP information was more likely to happen in some geographical locales than in others. One person, for example, compared Kentucky to New York by saying,

"A lot of people don't know about it for real, because like, the [inaudible] program I was in, I don't know about it and I'm 21 and I also feel like it depends on your location because he was from New York. I feel like maybe depending on 
where you're located because Kentucky is really, outside of Louisville I feel like most people wouldn't know about it." - Participant, LGBTQ+ mixed group Participants perceived that persons who live in less conservative places like New York would, by default, be more knowledgeable about PrEP compared to someone from Kentucky, a conservative state (although Louisville is politically liberal and thus expected to be relatively more open to sexual health promotion). This statement has implications for intervention development as it indicates that geographical location perpetuates disparities in exposure to sexual health promotion information such as PrEP.

\section{Perceived HIV risk and perceived need for PrEP}

Many participants did not perceive themselves to be at high risk of contracting HIV nor did they perceive themselves as having a need for PrEP. This low HIV-risk perception was observed across all groups but varied among participants, with heterosexuals expressing lower perceived risk and lower need for PrEP compared to participants in LGBTQ+ groups. For instance, several participants in the heterosexual groups felt that if they only had one partner or were in a monogamous relationship or if they were not having sexual intercourse with persons of the same sex, then they did not need to be on PrEP.

"I just kind of understand where these ladies are coming from. I look at it like if I have one partner and we're faithful, you know, we get checked every six months, we've got kids, you know I just don't feel it's for my situation. - Participant, heterosexual female-only group 
Another participant in this group also cited being in monogamous relationships as a reason for low perceived HIV risk. In addition, she believed that she was especially low risk by virtue of being a lesbian and not having sex with men.

“I wouldn't take it now because I'm also like in a monogamous relationship and I don't have sex with men, and even though lesbians are at risk for STDs and STIs and stuff like that, HIV is, for lesbians, it's kind of at a lower risk than it is for other people. So our, and I know our STDs are like herpes and HPV and, you know, those skin to skin contact ones, so with that and as long as we're getting checked regularly. Now if the situation changes where we want, where we decide to have sex with other people, even then it would probably not be PrEP, it just has to be more getting tested and opening up that line of communication." Participant, heterosexual female-only group

It should be noted that, this participant self-identified as a lesbian although this focus group was a heterosexual group. Since participants were screened into the study based on group identification and behavior, this could be an indication that the participant, though they identify as same-gender loving, may have had experiences with men in the past, or perhaps chose to identify as heterosexual female during screening. Other participants did not feel as though they were at risk because they were older (closer to 29 years).

"I think at this point in my life I would not get on it, at 28. But I think if I knew about it when I first went off to college I probably, that probably would have been something I did or I was on, because I was high risk. But I think at this point in 
my life no, but that's definitely something I would have done." — Participant, heterosexual female-only group

This downplay of HIV risk based on age appeared to be born from a perception that risky behavior decreases with age. The older participants perceived themselves to be at lower risk compared to when they were younger, such as during their early years in college when they were more likely to engage in behaviors that put them at risk for HIV. Given that both national and Kentucky HIV surveillance data indicates high incidence of HIV in 20-29-year-olds, this finding has implications for HIV risk reduction strategies. HIV interventionists may need to work closely with this population to better ascertain how the younger (lower half of the age range) compared to the older (upper half of the age range) conceptualize risk. This approach should help tailor risk reduction interventions for increased effectiveness with African American priority groups within this age range.

Participants within the MSM and LGBTQ+ groups, on the other hand, were more open to acknowledging their risk of HIV and hence their need for PrEP. Many participants in these groups believed that since they were sexually active, they needed to take the necessary precaution to prevent themselves from becoming infected.

"I don't. I don't look at it that way. I don't because everybody individually gonna have to use their own judgment. Okay, I'm sexually active, so let's go ahead and prevent this. Okay, let's do it...” — Participant, MSM mixed group "Yeah, 'cause I mean take a pill if I'm sexually active and it's gonna help me prevent HIV, why would I not take it?" — Participant, MSM mixed group 
MSM-identifying participants further acknowledged their need for PrEP citing their sexual preferences and tendencies to engage in high risk sexual behavior, such as not using condoms consistently or having multiple sexual partners. These participants were, however, aware that not using condoms puts them at risk, but they also alluded to the fact that not using condoms gave an increased sense of pleasure during sexual intercourse. Thus, they concluded that without some other form of protection, besides condoms, the potential for many individuals within their community to get sicker would increase. Thus, they concluded that PrEP would be a good option to prevent that from happening.

"But look you know what I'm saying, we don't want just be always with the rubber [condom or barrier protection], so why can't we just sometimes feel the skin to skin and in order to do that, we all need to, the whole united nation need to be on PrEP . If you think about it everybody will be sicker..." - Participant, MSM-only group

Although most MSM acknowledged their risk for HIV and need for PrEP, these individuals expressed anger and frustration about assertions of the health care system and consequent assumptions by society and that LGBTQ+ individuals are at higher risk for HIV and thus in greater need of PrEP compared to their heterosexual counterparts. LGBTQ+ participants believed that the HIV risk for heterosexual individuals was being underestimated and expressed indignation over targeting of PrEP towards LGBTQ+ individuals, especially towards gay and bisexual men. They believed PrEP should also be emphasized for heterosexual individuals whom participants believed were just at risk for HIV as were the LGBTQ+ individuals. 
"I'm saying to give women and straight [heterosexual] people. HIV is a scare to straight people. Like I said they don't think straight people get HIV." Participant, LGBTQ+ mixed group

Other participants in the MSM-only group expressed the same feelings about being perceived by society as the ones with the highest HIV risk. when, According to the MSM, heterosexual individuals are also at heightened risk, especially heterosexual men who have sex with men, but do not self-identify as MSM and should be blamed for complicating HIV risk for the MSM and larger LGBTQ+ community. Consider, for example, the following exchange:

Participant 2: "Like a stereotype they just automatically think gay people just have HIV and straight [heterosexual] people can't get it."

Participant 3: "And it's like when you all talk to us, you all are like, hey you know it's only for gay people like you have to be gay, bi, or lesbian."

These participants went as far as blaming other non-MSM-identifying heterosexual men, whom they referred to as "trades" (heterosexual men who secretly have sex with other men), for infecting MSM with HIV by having sex with MSM in addition to their heterosexual partners.

Participant 4: "It's the straight one that's really bringing the disease to us" Participant 3: "Yeah!! They're messing with us, I mean they come under the table"

MSM and other LGBTQ+ participants frowned upon this practice of "under the table" or "swinging" sexual behavior by heterosexual men who "move from one person to another." MSM participants exclaimed that this behavior puts the spouses, significant 
others, and even children of these individuals at risk. Here is a conversation among participants in one of the MSM-only groups:

Participant 2: "And then they go to their wives and they baby mamas and wo...wo...wo..."

Participant 3: "They got babies and they're giving it to them"

Similarly, other participants in a different LGBTQ+ group also share these sentiments. Not only did they think the "trade" or "swingers" were putting MSM at risk, but some also believed that the spouses of these individuals (namely, heterosexual women) were also just as responsible for increasing the risk of MSM. One participant stated:

"And like I said [crosstalk 00:24:25], HIV is the biggest thing for black women because black men don't go to the doctor. They just spreading stuff. Why? Because they move from one person to another, and more people are swingers. Sex is not a big thing anymore, it used to be a big ordeal but now it's just whatever"

This finding has implications for HIV prevention among African American men who do not self-identify as MSM. It is an indication that HIV prevention might not be adequately reaching these men and calls for interventionists to examine HIV risk-reduction strategies for adequately engaging African American heterosexual men who practice this behavior. Additionally, other participants recognized the need to embrace prevention to protect themselves, especially when they perceived that they may not have control over their sexual partner's sexual behavior. Thus, they expressed the need to be proactive in case a potential sexual partner chooses to be secretive about their sexual life, potentially putting them at risk of contracting HIV. 
"Well I mean honestly, you can't really say you're not at risk for HIV. There is so many ways to get HIV. And although you may be in a committed relationship, you just never know what your partner's doing. So, although your partner may be saying they're committed to you, let's say they go and do something off with another girl, not knowing that that girl has HIV. So, now you got HIV. Now you bringing it to me. And then let's say I'm cheating too. So, now I'm bringing it to the next person..." - Participant, heterosexual mixed group "Just know that it's a lot of funny business going around, and we have to protect yourself because, at the end of the day, some people can be secret about they sex life. So with that, it's even more scary because since you do have this man looking like a man, and here he goes somewhere else, dressing like a woman and then, bam, you feel me? He then comes back to his wife [crosstalk] You know what I'm saying? And then she's innocent, and then bam, she got AIDS because he's over here sneaking with men. You know what I'm saying? That's true. I see too much." - Participant, heterosexual mixed group

\section{Fears and reservations about PrEP}

\section{Misperceptions about PrEP eligibility}

Misperceptions about PrEP eligibility were prevalent among study participants.

They were either uniformed or misinformed about who is recommended to use PrEP. Many heterosexual-identifying participants, especially, believed PrEP was primarily for individuals who identify as LGBTQ+. This influenced participants' perceptions and predispositions towards PrEP. For instance, when asked if they would consider using 
PrEP, one heterosexual participant responded: "I mean, is it just for gay people or is it not?" Other participants in the study also shared this sentiment. For instance, when asked how many people had heard about PrEP and how they heard about it, some participants in the heterosexual groups said:

“Only people that I know personally who use it are gay men. I don't remember when I first heard about it but I know I've actually had a friend that brought it up in a conversation and said that he was using it so I kind of learned a little bit more about it, and I thought it was really dope so." — Participant heterosexual femaleonly group

Another participant also said:

"Yeah, that's kind of like the same thing for me. I heard about it a couple years ago at a conference and it was a conference that had a whole lot of LGBTQ people there, so that was one of the stations was like, PrEP, and basically for people who are at higher risk..." - Participant, heterosexual female-only group Participants insinuated that the misconception that PrEP was only for gay individuals was partly due to excessive targeting of PrEP advertisement towards LQBTQ+ persons through PrEP commercials. Participants believed that this practice perpetuates homophobia and PrEP-related stigma, as several individuals erroneously assume that PrEP is only for gay persons. As a result, participants expressed concern that heterosexual individuals would not see themselves as having a need for PrEP.

"And if you navigate the first thing you going to do heterosexual male or female you going to be like OK that ain't got shit to do with me..." - Participant, MSMonly group 
"Like you've mentioned, with the commercial when people see that they're going to automatically think oh, that's for gay people or that's a gay thing." -

Participant, LGBTQ+ only group

"It's okay like to have a couple gay men on there [the commercial], but when you look at the commercial and you see all gay people on there" - Participant, MSMonly group

\section{Fear of side effects and drug interactions}

The fear of side effects was one of the biggest concerns participants across all groups expressed about PrEP. Many participants were skeptical because of their lack of familiarity with the medication, especially its potential side effects. One person remarked, "I don't know all those side effects to that type of medicine, you know what I'm saying?" Some were apprehensive about how the PrEP medication would potentially affect their bodies and whether they would have any allergic reactions to it.

"I need to know what the side effects are if I put it in my body..." - Participant, MSM-only group

“Just 'cause you don't know the side effects that could actually happen. 'Cause certain people are allergic to certain medicines. 'Cause I know I can't put certain sunscreen on 'cause I break out. If I could just break out with sunscreen imagine what a pill can do to my immune system." - Participant, MSM and heterosexual male mixed group

Not only were participants concerned about the potential side effects of PrEP, but several of them were worried about possible drug interactions. They were particularly 
nervous about how PrEP might interact with alcohol or other medications for pre-existing or underlying conditions.

"Yeah because this is serious because I drink, so if I'm taking PrEP too, you know what I'm talking about, if I'm depressed and I decide that I wanna drink a half a gallon or two in the weekend or in a day or two, like, what is this gonna do to me being on PrEP?" - Participant, MSM-only group

"It's like the same thing with Adderall and stuff. People that have ADHD it's like you don't know if you wanna take that certain pill 'cause you don't know what the side effects of it. 'Cause there is side effects of it." - Participant, heterosexual mixed group

Even participants who had positive perceptions about PrEP still expressed fears as they speculated the side effects of the medication. This lack of awareness limited background knowledge about side effects and impacted participants' willingness to consider using PrEP.

"I think that it's definitely a good thing as well. In order for me, I feel like, to give my $100 \%$ feedback on, I would need to do more research on what it is exactly. I mean, I see the commercials, I've spoke with my doctor about it, but I don't know exactly what's in it. I don't know what the side effects may be, so I can't really say that I'm... I can't really root for it and I've not done my research for it yet." Participant, LGBTQ+ only group

Overall, participants were willing to use PrEP if they had enough information about its potential side effects and possible drug interactions and if they could assess that the side effects were mild or tolerable. 
"Yeah...if it was, if it could work, I mean and if the side effects weren't too bad... yeah, I'm big on that, I'll be listening to commercials and I'll just be hearing all of it like... I'm big on that." - Participant, male-only group

\section{Adherence beliefs}

Adherence was a deterrent for willingness to use PrEP among many participants. Several participants expressed a dislike for taking medications in pill form and thus questioned their personal motivation and ability to adhere to taking PrEP consistently every day. Many also did not feel confident they could remember to take it religiously. "Because it's like I have a hard time with that medicine stuff." — Participant, heterosexual male-only group

"I'm just too forgetful. I would not take it every day." — Participant, heterosexual mixed group

"And I would say I'm right in the middle of you two. I really don't take that much medicine and, for it to take it with extra medicine for something that I personally in my head think I wouldn't get, I wouldn't really be motivated to take it every day." - Participant, heterosexual mixed group

PrEP was also likened to birth control and prenatal medications, both of which are required to be taken daily for optimal results. Participants cited previous personal experiences as well as experiences of their peers or their partners having difficulty adhering to birth control or prenatal pills. Thus, participants assumed PrEP would be just as difficult to adhere to, if they were to be prescribed the medication.

“That's why I got a baby. It's hard for everybody. Know what I'm saying, like my baby mama can't even take her birth control, know what I'm saying, like, it 
ain't even like, she don't mean to, know what I'm saying? But it's just like, know what I'm saying, and we might not even be...know what I'm saying...doing nothing...know what I'm saying! But you gotta take it.” — Participant, heterosexual male-only group

While most participants questioned their ability to adhere to a daily PrEP regimen, others, albeit only a few, were confident in their ability to take a daily PrEP pill.

"I would rather take a pill than get that shot. I'll rather take a pill. I ain't gonna forget... sitting in my room on my little dresser, it will be the first thing I do when I wake up in the morning."- Participant, MSM-only group

\section{Acceptability of PrEP}

\section{Acceptability of PrEP as a viable HIV prevention approach}

Acceptability of PrEP as a viable HIV prevention approach varied among participants. Some were in support of the idea and others were skeptical for various reasons. While many participants were not keen on the idea of taking a daily medication (pill) like PrEP to prevent HIV, some participants across the various groups expressed positive predispositions towards the concept, and several participants felt it was a welcome intervention for the prevention of HIV.

"I thought that it was a great idea actually. Something to prevent something from happening I just think that that's smart. It's one step ahead and it's pretty awesome. I've never heard any (negative) feedback about PrEP so." Participant LGBTQ+ mixed group

Another person also noted: 
"I feel like if you have PrEP or what not, I feel like that it would prevent the chances of a lot of people catching AIDS and HIV. So, I feel like, yeah, I feel like PrEP is a big thing." — Participant, heterosexual mixed group

Some also saw PrEP as a major step in the right direction and were hopeful of the possibility of finding a cure soon for HIV.

"I think it's awesome, because I think with all of the research and all the money that's put into the HIV thing, it should be some progress like that. It should even almost be a cure, I'm sure, somewhere around the corner. Hopefully. So, I think this is a giant leap, but I think this is the beginning of something better for this situation" - Participant LGBTQ+-only group

While many saw PrEP as a welcome intervention, other participants had doubts about its efficacy and viability in the long term. Some participants, for instance, did not believe that the producers of PrEP had adequate knowledge about the drug's effectiveness, particularly in the long term.

“I don't know nothing about it. I don't think the people who's making it know everything yet either. So, they don't really know the 20 -year effects of it." Participant, MSM \& heterosexual male mixed group "20-year mark, like he was saying earlier. It takes a long time 'til you pick up different signs of things." - Participant, MSM \& heterosexual male mixed group Others simply did not have faith in the medication. They were fearful that instead of preventing HIV, PrEP might instead cause them to become infected with HIV or to develop another condition like cancer. 
"I'm scared that it might backfire. What if it instead of prevent AIDS, it gives you AIDS? That's what I was thinking all the time like, 'Okay you don't have to have AIDS to take it but what if it just triggers the cells that cause cancer and stuff?" Participant, heterosexual mixed group "I'm saying, 'Oh yeah, it [PrEP] causes you not to have HIV' like that, but at the end of the day, if I got that, then you all must be careful with it, because that's crazy." -Participant, heterosexual mixed group

\section{Acceptability of PrEP in its current pill form}

Most participants liked the idea of having a medication to prevent HIV, but many participants expressed dissatisfaction with PrEP in its current form (available only as a pill).

"I don't like pills, so if it's pills I don't like pills. I don't know how to take pills."

- Participant, heterosexual female-only group.

“Yeah, I don't. I'm not really a big medicine fan. I don't like, I don't wanna have to take no pills, I don't really like to take no pills especially if I don't have to." Participant, heterosexual male-only group.

Heterosexual women, especially, lamented over the lack of options to choose from as is the case with birth control medications. They felt strongly that PrEP should be available in multiple forms to account for variations in medication preferences among individuals.

One participant noted:

"But that's why it's important to have those alternatives. Some people are very good with pills, and then other people need ten years, some people need three 
years, five, other people need a ring, some people are scared of needles so they need, you know, it's so many different things" — Participant, heterosexual female-only group.

Overall, there were mixed preferences among study participants regarding what form PrEP medication should take — that is, whether it should be a pill, a shot, or some other form. Preferences of medication form influenced willingness to use PrEP, as some participants indicated they would only be willing to use PrEP if it were available in a form other than a pill.

"And I think y'all should like turn it into like a liquid form or like a powder form like he said too, something to shake up. Yeah, I think pills is old, pills is just like old people thing, that's what that was." - Participant, heterosexual male-only group

Across the various groups, arguments ensued among participants regarding duration and frequency of taking PrEP. Many wondered if it were possible to change the dosage frequency from daily to biweekly or monthly, to improve adherence. Most participants preferred a shot and suggested that PrEP should be made available in an injectable form and on a less frequent basis. They did not feel like others like them would be accepting of PrEP in its current pill form.

"It's not only the, I think that's the two things like... maybe if it was like, and I ain't trying to switch it over, like maybe if it was like a shot or something" Participant, heterosexual male-only group "But see I feel like if you take it once a month or once every two weeks maybe, a shot, that you ain't got to worry about it because it's already in your system, and 
then you already know 'I done had it, I ain't got nothing to worry about, I can live my life and do what I wanna do"” — Participant, MSM-only group "Yeah... that's what I was going to say, like an annual shot or something." Participant, heterosexual male-only group

"I think you should take the PrEP [as] a shot and for once a month or so, you know what I'm saying? Like a regular pill. They ain't going to remember to take no pill though." — Participant, heterosexual mixed group

While most participants were against having PrEP only available in pill form, others were satisfied with PrEP in its current form and indicated that they would be willing to use it even as a pill.

"I would rather take a pill than get that shot." — Participant, MSM-only group “But I know I take my pill every day." — Participant, heterosexual mixed group

\section{Discussion}

The purpose of this study was to develop a deeper understanding of intrapersonal factors influencing PrEP engagement and uptake among African American priority groups, ages 18-29 years old. Study findings highlight the impact of PrEP knowledge, perceptions, and attitudes on the intention to engage with or use PrEP by young African Americans who practice high-risk behaviors. The findings were in alignment with the predictions of the TRA/TPB (Ajzen, 1991) from which two constructs were utilized as sensitizing concepts for designing the study focus group guide. That is, participants with more positive perceptions and attitudes towards PrEP were more likely to indicate willingness to use PrEP. Also, perceived behavioral control factors such as level of knowledge, awareness, and understanding of PrEP's effectiveness as well as perceptions 
about potential side effects, drug interactions, and beliefs about adherence influenced predispositions towards PrEP (i.e. willingness to engage with and use PrEP or not). Other studies also demonstrated influence of attitudes and perceptions on wiliness to use PrEP (Collier et al., 2017; Eaton et al., 2014; Smith, Toledo, Smith, Adams, \& Rothenberg, 2012).

Also in line with findings from previous research among African Americans, this study revealed low PrEP awareness and knowledge among most participants (Cahill et al., 2017; Eaton, Driffin, Bauermeister, et al., 2015; Eaton et al., 2014; Eaton, Matthews, et al., 2017; Strauss et al., 2017). Most participants in the current study had never heard about PrEP prior to the study, and the few who were aware of PrEP only possessed marginal PrEP knowledge. It is also important to note that in this current study, individuals identifying as MSM or other LGBTQ+ demonstrated more PrEP awareness, relative to heterosexual identifying individuals. Only a few MSM or LGBTQ+ individuals in this current study reported previous or current PrEP use. This could be due to our gay participants being connected to several other gay individuals and thus sharing information about HIV prevention among one another. Social network size (that is, being connected to larger number of other young MSM) has been positively associated with PrEP use among young MSM (Kuhns, Hotton, Schneider, Garofalo, \& Fujimoto, 2017). Another reason for more willingness to use PrEP among MSM could be that PrEP promotion is only reaching a small segment of the population, namely LGBTQ+, who may be more exposed to PrEP information within their communities as a result of targeted PrEP advertisements towards gay individuals. This finding suggests that more interventions, such as PrEP education campaigns targeted towards a wide variety of 
young African American priority groups, are needed to increase PrEP awareness and knowledge among this population. Prior to the study, no mass media campaigns targeting various African American priority groups had been implemented in this region.

Additionally, participants who identified as heterosexuals (both men and women) showed lower levels PrEP awareness. This was especially more surprising to see that women were unaware since African American women typically have more knowledge of health-related issues. This finding has implications for PrEP use since PrEP knowledge and awareness have been previously shown to impact PrEP engagement and wiliness to use PrEP among heterosexual African American priority groups, especially women (Auerbach et al., 2015; Collier et al., 2017).

Another interesting finding was the low perceived risk of HIV and low perceived need for PrEP among participants also reported in other studies (Elopre et al., 2018; Smith et al., 2012). While all participants self-reported engagement in high-risk sexual behavior, which was the criteria for enrollment into the study, many, especially heterosexual individuals, underestimated their risk of HIV and, hence, their need for PrEP. Some believed that because they were in monogamous relationships or were not having sexual relations with a person of the same sex, then they were not at risk for HIV and, thus, PrEP was not applicable to them. Individuals sometimes underestimate their risk by believing they are in monogamous relationships when in reality they may be engaging in unprotected sex with partners who may not be practicing monogamy (Centers for Disease Control and Prevention, 2018f). Other women, however, acknowledged their risk for HIV and saw PrEP as a welcome prevention option, especially in instances where their sexual partner was not practicing safe sex. In previous 
focus groups with African American women, PrEP has also been considered as a welcome option for HIV prevention, as it gives women more control when they cannot control their partner's behavior or in cases where barrier protection fails (Collier et al., 2017; Flash et al., 2014; Smith et al., 2012). Optimizing willingness to use PrEP by heterosexual individuals has implications for HIV prevention among African American heterosexual women, especially since $86 \%$ of HIV cases among women are attributed to heterosexual sexual contact (Centers for Disease Control and Prevention, 2018f).

Furthermore, participants voiced several concerns about PrEP in varying degrees. For instance, participants had mixed reactions about taking a daily pill for preventing HIV. Some questioned their own confidence in adhering to a daily regimen. Others did not like pills and thus desired more options for PrEP, such as injectables. This concern for taking a pill every day has been reported in previous studies where minority MSM (African Americans included) were more likely than White MSM to express concerns about having to take a pill every day (Lelutiu-Weinberger \& Golub, 2016). This should be taken into consideration for PrEP implementation as it has implications for PrEP adherence.

Of all the concerns expressed by participants, fear of side effects was the most prevalent across all focus groups. This concern was so strongly felt that it had considerable impact on PrEP-use intentions among this sample. Most participants indicated they would only be willing to use PrEP if they had enough information about its side effects and were convinced that PrEP would not negatively impact their health in the long term. This intense fear of side effects and its corresponding impact on willingness to use PrEP has also been reported in other studies (Cahill et al., 2017; Collier et al., 
2017; Smith et al., 2012). In one of these studies, even though PrEP knowledge was not statistically associated with being African American (meaning African Americans had comparable levels of knowledge as others in the study), young African American MSM (along with Latinos in the study) were more likely than Whites to decline interest in PrEP-use due to fear of side effects. Participants desired upfront information about potential side effects and long term impact of PrEP on health suggested that PrEP education should be explicit about these along with other pertinent information like efficacy and potential drug interactions. These findings have significant implications for PrEP implementation among young African American priority groups. Future research should consider assessing tailored strategies for addressing root causes of fears, stigmas, and misconceptions relating to PrEP-use among African Americans, as these have the potential to significantly impact uptake of PrEP among this population.

Overall, participants were accepting of PrEP as a good option for preventing HIV and were willing to engage with it and even use PrEP provided they were knowledgeable about it, had enough information about it, and were not kept in the dark about their many fears and reservations regarding PrEP. This has implications for practice. AIDS service organizations and health promotion specialists should take these concerns into consideration when designing promotional materials targeting African American clients. Given that willingness to engage with and use PrEP by African American priority populations hinges on many concerns that appear to have been created either directly or indirectly by the health care system, it behooves the health care system to take responsibility for its actions and begin correcting some of those unintended consequences of well-intentioned interventions. A good place to start is to begin working with African 
American communities to remedy PrEP awareness campaigns and other interventions like it that may have been misperceived as perpetuating stereotypes and stigmas against sexual and gender minorities and, hence, alienating and minimizing HIV risk among sexual and gender majorities. These interventions should endeavor to be open, honest, and transparent and focus on explicitly addressing the population's various concerns about PrEP, especially potential side effects. The fear of side effects appeared to be the biggest source of concern among participants and had considerable impact on willingness to use PrEP. Developing a tailored PrEP campaign with input from the community should serve two purposes: (1) to dispel stigmas and misconceptions that PrEP is only for certain individuals and (2) to create more awareness within the community. The sooner health promotion interventionists acknowledge and adequately address the various intervention shortfalls, the sooner will some barriers be broken down among African Americans that preclude engagement with biomedical interventions like PrEP.

\section{Conclusion}

Disparities in HIV rates among African Americans, particularly among young priority groups (e.g. MSM, LGBTQ+, and heterosexual-identifying individuals demonstrating heightened sexual risk behaviors - history of non-condom use, multiple concurrent sexual partners) warrant high-impact, biomedical prevention initiatives like PrEP. It was evident from the study that increased PrEP awareness and knowledge are likely to yield increased engagement in PrEP and willingness to use PrEP by African American priority populations. However, participants indicated that in addition to increasing PrEP awareness, they were interested in more upfront information about potential side effects, effectiveness, and potential drug interactions. Overall, participants 
were more willing to consider using PrEP if they were presented with comprehensive information and if PrEP was made available in other options, in addition to its current pill form. Furthermore, findings uncovered low perceptions of HIV risk and low perceived need for PrEP among heterosexuals. Since there is a paucity of research on PrEP uptake among African American heterosexual individuals, especially women, future studies should consider examining determinants of PrEP use and PrEP engagement among African American women as well as heterosexual males not practicing safe sex behaviors. Finally, researchers, AIDS service providers, and health promotion interventionists should consider developing culturally tailored interventions to address low PrEP awareness, knowledge, and other pressing concerns such as PrEP efficacy and side effects among African Americans to increase willingness to engage with and use PrEP for HIV prevention. 


\section{CHAPTER V: PAPER 2}

\section{BEYOND THE INDIVIDUAL: EXAMINING SOCIOECOLOGICAL AND STRUCTURAL INFLUENCES ON HIV PRE-EXPOSURE PROPHYLAXIS (PREP) ENGAGEMENT AMONG YOUNG AFRICAN AMERICANS IN KENTUCKY}

\section{Introduction}

The previous chapter partially addressed the first research question: What are the multi-level barriers and facilitators to PrEP engagement, from the perspectives of African American youth groups in Louisville, KY who are at high risk for HIV? It also fulfilled study aim one, to explore and understand barriers and facilitators to, and engagement with, PrEP uptake among African American priority groups. Specifically, the chapter provided partial evidence — individual level/intrapersonal facilitators or barriers to PrEP engagement and uptake — derived from focus groups with young (18-29-year-old) African Americans residing in Louisville, KY who are at heightened risk for HIV.

This current chapter complements the previous chapter. While it was important to explore, and understand intrapersonal factors impacting individual engagement with PrEP, individual-level behavior predictors alone may be insufficient to fully explicate reasons behind unwillingness to engage with PrEP by African American youth demonstrating heightened HIV risk. In fact, evidence suggests that factors beyond individual control may influence behavior, such as interpersonal, social, economic and 
cultural contexts which may restrain or promote such behaviors (DiClemente et al., 2007). Therefore, it was imperative to also explore higher-order factors, transcending individual behaviors, that may further explain reasons behind African American youths' low engagement with PrEP. Thus, the manuscript presented in this chapter focuses on

sociocultural, societal, and structural factors impacting PrEP engagement among African Americans groups practicing high-risk behaviors derived from focus groups with young (18-29-year-old) African Americans residing in Louisville, KY who are at heightened risk for HIV.

\section{Background}

African Americans have consistently exhibited the highest rates of newly diagnosed HIV cases annually in the U.S. (Centers for Disease Control and Prevention, 2019d). Despite comprising only 13\% of the U.S. population, African Americans accounted for $43 \%$ of all new HIV cases diagnosed in 2018 (Centers for Disease Control and Prevention, 2018b). This disproportionality in HIV disease burden among African Americans is especially apparent in young men who have sex with men (MSM), bisexual men, and women (Centers for Disease Control and Prevention, 2018c, 2018f). Further, the majority of persons newly diagnosed with HIV in 2018 reportedly lived in the South (Centers for Disease Control and Prevention, 2020a).

A high-impact HIV intervention, PrEP, was approved by the FDA as a daily oral drug in 2012 as Truvada and, more recently in 2019, as Descovy for prevention of HIVnegative individuals practicing high-risk behaviors (U.S. Food and Drug Administration, 2012, 2019). PrEP has demonstrated effectiveness in decreasing new infections in various priority groups and holds promise for helping to end the HIV epidemic (Baeten et al., 
2012; Choopanya et al., 2013; Grant et al., 2010; McCormack, Dunn, Desai, Dolling, Gafos, Gilson, Sullivan, Clarke, Reeves, Schembri, et al., 2016; Sheth, Rolle, \& Gandhi, 2016). However, evidence suggests disparities in PrEP engagement and uptake among youth, women, and African Americans (Buchbinder \& Liu, 2018, 2019). For instance, national prescription data shows that fewer African Americans compared to Whites have used PrEP to date, and PrEP was prescribed the least in southern states (Bush et al., 2015; Siegler, Bratcher, et al., 2018). These findings are further supported by research demonstrating higher PrEP uptake and engagement among White MSM compared to African American MSM (Kuhns et al., 2017).

PrEP knowledge, awareness, perceptions, and attitudes have been shown to impact engagement with and uptake of PrEP among various populations including African Africans (Al-Tayyib, Thrun, Haukoos, \& Walls, 2014; Bauermeister, Meanley, Pingel, Soler, \& Harper, 2013; Dolezal et al., 2015; Eaton, Driffin, Bauermeister, et al., 2015; Elopre et al., 2018; Koechlin et al., 2017; Mimiaga, Case, Johnson, Safren, \& Mayer, 2009; Mutchler et al., 2015; Smith et al., 2012). Research further shows that African Americans, compared to Whites or Latinos, are more likely to have unfavorable predispositions towards PrEP use, hence suboptimal levels of PrEP uptake among African American groups (Eaton, Kalichman, et al., 2017; Lelutiu-Weinberger \& Golub, 2016).

While intrapersonal factors are important predictors of behavior, evidence suggests that individual behaviors are influenced by various factors within people's social and physical environments which may restrain or promote those behaviors (DiClemente et al., 2007). Thus, individual factors alone are insufficient for explaining behavior or the 
disparities in health outcomes across populations that may be based on their race, social status, class, gender, or sexual orientation. There are underlying systemic root causes of disparities referred to as social determinants of health (SDOH). These are conditions in which people are born, grow up, live, work and age, and the systems put in place to deal with illness, which are then influenced by policies, economic, social, and political forces (World Health Organization, 2016).

SDOH such as living in disadvantaged settings, income, education, housing, poverty, unemployment, racial/ethnic segregation, and access to health care services been linked to health outcomes like HIV risk (Adimora \& Auerbach, 2010; Cene et al., 2011; Gant et al., 2014; Kahana et al., 2016; Viner et al., 2012). Similarly, social capital, poverty, and income inequality predict AIDS rates and other sexually transmitted diseases (STDs) in the U.S. (Holtgrave \& Crosby, 2003). This is especially important for African Americans because, for this population, the interaction between factors such as where they live and work, as well as the resources available to them, may influence their risks of exposure to diseases like HIV/AIDS as well as access to health services. Some SDOH impacting HIV among African Americans have been documented. These include, but are not limited to, racial and ethnic segregation (Kahana et al., 2016), lack of trust in the health care system, conspiracy beliefs (Bogart \& Thorburn, 2005), socio-economic factors (e.g. housing and means of transportation), high incarceration rates (which influence sexual networks), HIV-related stigma (Adimora \& Schoenbach, 2005; Eaton, Driffin, Kegler, et al., 2015; Kerr \& Jackson, 2016; Kerr, Valois, DiClemente, et al., 2015; Reif et al., 2014), and homophobia and homonegativity (Adimora \& Auerbach, 2010). 
Consequently, stigma, medical mistrust, cultural beliefs, cost, interactions with health care providers, and PrEP availability and accessibility have also been shown to have significant implications for PrEP uptake and engagement among African Americans (Eaton et al., 2014; Eaton, Kalichman, et al., 2017; Elopre et al., 2017; LelutiuWeinberger \& Golub, 2016; Wingood, Dunkle, et al., 2013). Research evidence further demonstrates that sociocultural, socioeconomic, and systemic/structural factors are often far more important determinants of PrEP use and willingness to use PrEP among African Americans than individual factors like knowledge and awareness (Eaton, Kalichman, et al., 2017). Researchers call for more investigations emphasizing a better understanding of multi-level factors that influence PrEP access and uptake among African American highrisk populations (Elopre et al., 2017; Lelutiu-Weinberger \& Golub, 2016; Mutchler et al., 2015).

Sociocultural and systemic factors have a significant impact on willingness to engage with and use PrEP among African Americans. These multi-level determinants have not been widely explored among African Americans in various risk categories beyond MSM. Most studies with African Americans focused mainly on MSM and bisexual males (Eaton, Driffin, Bauermeister, et al., 2015; Eaton, Driffin, Kegler, et al., 2015; Eaton et al., 2014; Mutchler et al., 2015; Philbin et al., 2016). Only a few studies focused on heterosexual women and men practicing high-risk behaviors (Auerbach et al., 2015; Collier et al., 2017; Flash et al., 2014; Smith et al., 2012; Wingood, Dunkle, et al., 2013) and even fewer studies explored these factors among African American transgender persons (Eaton, Kalichman, et al., 2017; Eaton, Matthews, et al., 2017). To our knowledge, this is the first study of its kind to assess these factors among a 
multiplicity African American priority groups in Kentucky. Thus, to add to this knowledge base in literature, this study explored multi-level barriers and facilitators to PrEP engagement and uptake among heterosexual men and women, in addition to LGBTQ+ individuals. Understanding multi-level reasons behind reticence to PrEP use and engagement among African Americans in various priority groups is a necessary step towards developing effective measures to reduce disparities in PrEP use among vulnerable African American groups and ultimately curb this epidemic.

\section{Methods}

The AFYA PrEP study was informed by the CGT method (Charmaz, 2014), which has its philosophical underpinnings in symbolic interactionism (SI) (Blumer, 1986) and pragmatism (Lewis, 1976). SI assumes that meanings and actions are formed and shaped by language and symbols. That is, people act towards any given situation based on the how they interpret it, and the meanings ascribed to the situation may, in turn, be modified by the situation (experiences and encounters) (Blumer, 1986; Charmaz, 2014). CGT allows for theoretical sensitizing concepts, thus, the Social Ecological Model was utilized as a sensitizing concept to explore and understand multilevel factors serving as facilitators or barriers to PrEP engagement or PrEP use among young African Americans.

The AFYA team conducted 11 focus groups $(\mathrm{N}=63)$ (table 3$)$, with participants ranging between 18-29 years old and who self-identified as (i) men who have sex with men (MSM), (ii) transgender females, (ii) LGBTQ+, and (iii) heterosexual individuals practicing high-risk behaviors. Participants were recruited through local churches, LGBTQ+ balls, LGBT pride festivals, parties, local community-based organizations (CBOs), a Syringe Exchange Program (SEP), grocery stores, a local university, the local 
health department, and community events; a hired recruiter to help recruit the hard-toreach groups (LGBTQ+); and by direct referral of peers through the method of respondent driven sampling (RDS).

Team members, a majority of whom identified as African American, were trained and experienced in qualitative data collection. Two team members conducted focus groups in ( one facilitated while the other took notes). Facilitators employed a semi-structured focus group guide. Focus groups were audio recorded and lasted approximately 60 minutes. Informed consent was administered to all study participants before focus group data collection commenced. Focus group recordings were transcribed verbatim by two team members and a transcription service (Rev.com). Identifying information was removed and transcripts were coded using Dedoose qualitative analysis software (Dedoose.com).

Table 3 Focus Group Sample Description

\begin{tabular}{|l|l|l|l|}
\hline Group & Group Composition & $\begin{array}{l}\text { Focus } \\
\text { Group ID }\end{array}$ & $\begin{array}{l}\text { Number of } \\
\text { Participants }\end{array}$ \\
\hline 1 & MSM-only & MS1 & 3 \\
\hline 2 & MSM \& heterosexual male mixed group & MS2 & 10 \\
\hline 3 & LGBTQ+ only Group & LG1 & 4 \\
\hline 4 & LGBTQ+ \& heterosexual male mixed group & LG2 & 5 \\
\hline 5 & Heterosexual male- only & HM & 3 \\
\hline 6 & Heterosexual female-only group 1 & HF1 & 7 \\
\hline 7 & Heterosexual female only group 2 & HF2 & 4 \\
\hline 8 & Heterosexual mixed-gender group 1 & HMG1 & 4 \\
\hline 9 & Heterosexual mixed-gender group 2 & HMG2 & 7 \\
\hline 10 & Heterosexual mixed-gender group 3 & HMG3 & 9 \\
\hline 11 & Heterosexual mixed-gender group 4 & HMG4 & 7 \\
\hline N & Total Number of Participants & & 63 \\
\hline
\end{tabular}

Constructivist grounded theory analytic techniques (initial, focused coding)

(Charmaz, 2014), an evolved version of traditional grounded theory methods (Strauss \& 
Corbin, 1994) along with thematic coding (Strauss \& Corbin., 2015), were utilized to generate themes. Initial coding was completed on four out of 11 transcripts that allowed codes to emerge from the original data. These codes were combined and grouped into focused codes that were clearly defined to highlight the underlying properties of each code. Two members of the team (RC and SA) worked independently and together to refine codes and negotiate code definitions to arrive at a finalized version of the codebook (with focused codes). Some sub-codes were derived to help create subcategories within the main focused codes in order to provide an in-depth analysis of the data. A finalized codebook was developed in multiple iterations and was reviewed and agreed upon by two team members (J.S and SA). A pooled kappa score of 0.90 indicated excellent inter-rater agreement among coders (Cohen, 1960). The codebook was used to code all transcripts ( $\mathrm{N}=11)$ using Dedoose, a qualitative analysis software (Dedoose.com).

\section{Results}

All focus group participants ( $\mathrm{N}=63$ ) were African Americans between the ages of 18-29 years old and resided in Louisville, KY. For the purposes of this current analysis and corresponding results, focus groups broadly comprised of a) MSM only (N=3), b) heterosexual male only $(\mathrm{N}=3)$, heterosexual female only $(\mathrm{N}=11)$, c) LGBTQ+ (sexual and gender minority) only (N=4), d) two groups of MSM, LGBTQ+, each mixed with other heterosexual males $(\mathrm{N}=15)$, and e) mixed heterosexual males and females $(\mathrm{N}=28)$. Comparisons were made across broad groups to highlight significant differences observed across risk categories. 
Study findings revealed that interpersonal relationships and sociocultural issues like stigma, homophobia, and homonegativity influenced participants' predispositions towards PrEP. Many participants insinuated that people within their community had poor views of HIV and thus would not view PrEP use in a positive light. Thus, participants were concerned about what other people would think of them if they were using PrEP. As a result, most participants were only willing to use PrEP if they perceived that their social network or significant others or family members approved of PrEP. Stigma was expressed in the form of judgmental views and attitudes towards persons who have HIV and persons taking a medication intended to prevent HIV as well as associating PrEP with gay individuals. These stigmatizing attitudes appeared to be born from cultural norms of homophobia, homonegativity, and negative beliefs about HIV. These factors, along with medical mistrust, were reported as barriers to PrEP engagement and use. Systemic and structural factors such as cost of PrEP medication, insurance coverage, availability and accessibility of PrEP at primary care settings, and responses to PrEP engagement strategies of interventionists, like AIDS service organizations, were expressed as potential facilitators or barriers to PrEP engagement and uptake. Based on these findings, several themes emerged from the data (Figure 13) organized in five categories: (a) opinions of referents, (b) medical mistrust, (c) stigma, (d) PrEP availability and accessibility, and (e) interventionists' PrEP engagement strategies.

\section{Opinions of referents}

Many participants demonstrated that the experiences and opinions of others such as peers, family, friends had the potential to positively or negatively influence participants' intentions to use PrEP. For instance, participants indicated that they would 
be unlikely to use PrEP if other people within the African American community did not endorse or use PrEP.

"I feel like if a lot of African Americans aren't using it as much, then why would everybody else use that's African American too? If our population around here's not using it as much, then why would we just start doing it out of nowhere not knowing nothing about it?" - Participant, MSM \& heterosexual mixed group.

Figure 12 Facilitators or barriers to PrEP engagement and uptake among priority high risk groups.

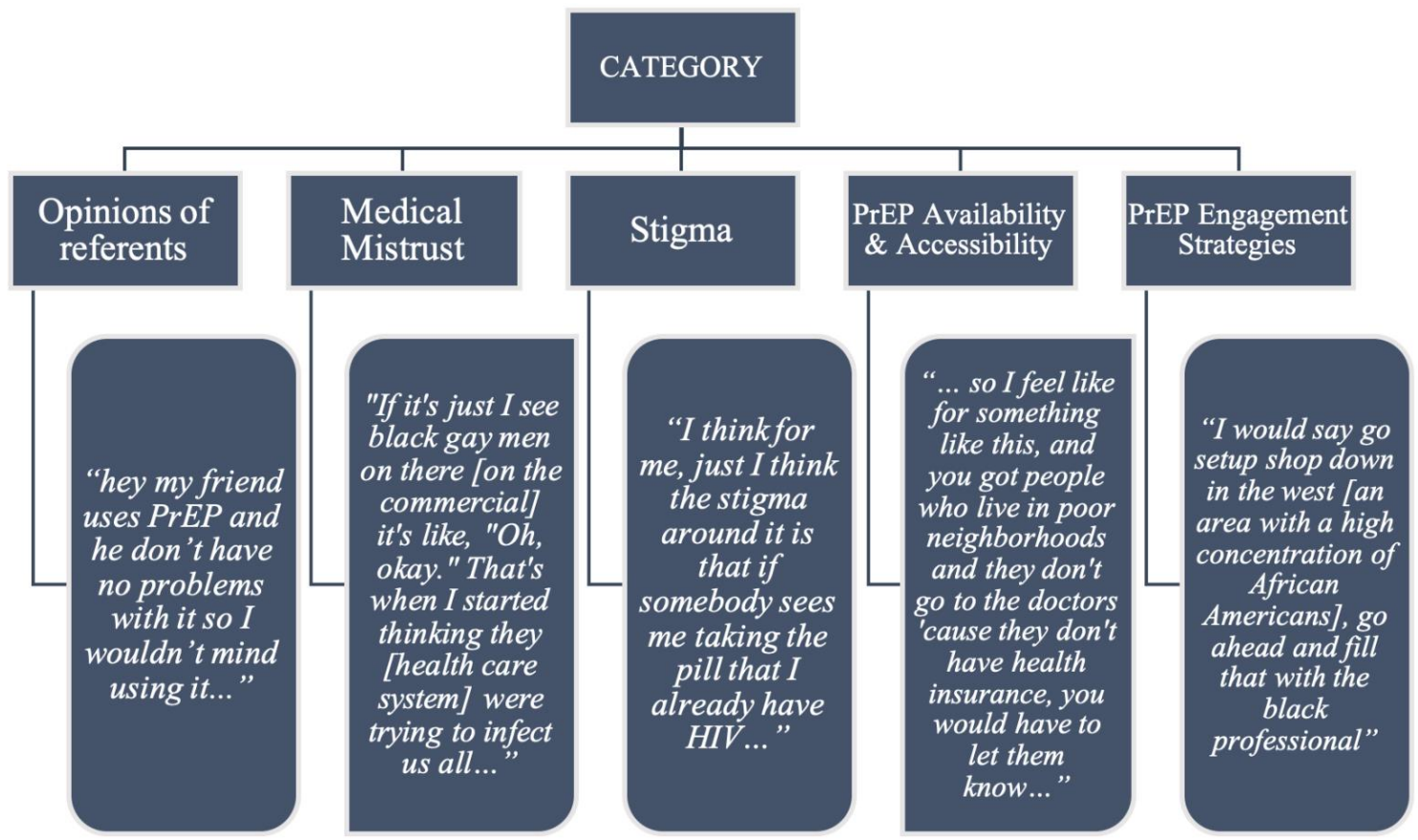

Thus, participants' lack of willingness to use PrEP might be indicative of the landscape of PrEP within the larger African American community. It is possible that the intentions to not use PrEP observed among participants are born from a profound lack of familiarity with PrEP across the larger population.

Consequently, while there were a few outliers who did not care what others thought of them, many participants in the study demonstrated that opinions and personal experiences of others whom they trusted or cared about had a direct bearing on their 
willingness to engage with PrEP for HIV prevention. For instance, some indicated they would be more willing to use PrEP if they saw that people they identified with and trusted were using PrEP and those people recommended PrEP to the participant. One participant remarked, "But if my people's doing it and they telling me, I trust them. I'm like, "Okay I'll consider this, I'll look into it more." Similarly, another participant reported that a friend of theirs was using PrEP and did not have any issues. Therefore, the participant indicated willingness to use PrEP as well. They stated, "hey my friend uses PrEP and he don't have no problems with it so I wouldn't mind using it..."

Participants also demonstrated that the judgmental opinions of peers, friends, family, and significant others could be a deterrent to using PrEP. Some participants expressed that their referents might be offended if they knew the participant was taking a pill to prevent HIV:

"Well, if you're with someone and it's supposed to be just you two, and you're taking an HIV pill, they may be offended." - Participant, heterosexual femaleonly group

“There's just so much judgment with any medication. Even with my birth control, I got the prescription because of ovarian cysts, so I always feel like I have to defend myself when I'm talking about it." — Participant, heterosexual femaleonly group "Because of how people, so I just feel like with any medication that's related to anything that's like sexuality related, and I know that's the only way you can get HIV, but that's the most common way. Even if you're just trying to protect 
yourself, people make assumptions." — Participant, heterosexual female-only group

\section{Medical Mistrust}

Mistrust of PrEP was prevalent among participants in the focus group, especially among male participants. Some were skeptical because they perceived the intervention was trial of some sort to test the experimental drug, PrEP, on some individuals. This leeriness of research and the fear of being a "guinea pig" (test subjects) was a deterrent to accepting PrEP for the some participants.

"I don't want to be a guinea pig or something like that..." - Participant, MSMonly group

"I don't want you like to try it and be like OK so we're going to see what's going on...”- Participant, MSM-only group

"So as far as this PrEP goes right, so say I wanted to be just, you know certain people, it's also a test for certain people, right? Am I lying? I mean because, think about it, right, they are only giving you this to see your reaction, to see your behavior separate from not being on it and being on it, so I am already a whore and I wanna go out here and whore..." - Participant, MSM-only group Mistrust of PrEP was also expressed in terms of aspersions towards PrEP advertisements. Participants were leery of the practice of excessively targeting gay and other LGBTQ individuals with PrEP commercials. They interpreted this practice as an attempt by the health care system to infect African American gay men with HIV. This misconception further cemented participants' conspiracy belief that HIV was man-made. 
"But I think too, with it being everywhere too... it's just targeting me as a black gay man. If it's just I see black gay men on there [on the commercial] it's like, "Oh, okay." That's when I started thinking they [health care system] were trying to infect us all. - Participant, heterosexual and MSM mixed group. "That what I want to say. But why is PrEP only offered to gay people? Why not straight people?" - Participant, MSM-only group

“Like it scares me that they're only targeting towards gay people, so, is this [HIV] a man-made thing?" - Participant, MSM-only group

Along with mistrust of the PrEP medication, some participants expressed misconceptions and endorsed myths and conspiracy beliefs about HIV, which impacted how participants viewed PrEP. For instance, some participants endorsed the belief that HIV and other chronic diseases like cancer are not real, but rather made by a person and injected into people. One participant stated, "is a lot of stuff that is man-made." Another participant responded, "yeah from what I heard...it was [they] injected it..." and another added, "I mean HIV, cancer and all that shit is man-made that's my opinion."

Others believed that the medical community has a cure for HIV and a cure for cancer, but that cure is expensive and is being hoarded by the medical community to make merchandise of people.

"I just don't get why they don't have a cure for HIV. I feel like they [do]. You know what I'm saying? I feel like they know that cure." - Participant, heterosexual mixed group "They do have a cure. I heard it costs a lot of money." - Participant, heterosexual mixed group 
"These kids have cancer and you got a cure for cancer, but you won't give it to them because you want the money. You're thinking about the money. You're thinking about all the medicine ... It's like saying, you're seeing these kids suffer. You feel me?" - Participant, heterosexual mixed group These misperceptions and mistrust of health care systems impacted participants' views of PrEP and their willingness to use PrEP to the extent that participants, especially males, did not trust their doctor's advice about PrEP.

"Yeah, my doctor just kinda brought it up to me. He was just like, 'Are you sexually active?' And I was like, 'Yeah.' And instead of him asking my lifetime number, which is what I'm used to kinda hearing, he was just like, 'Are you on PrEP?' And I was like, 'No, and I don't know about getting on it.' Cause I was still kinda leery about it. So, he's kinda got the ball rolling on it. I still have some reservations. Just about, I don't trust a lot. So that's just my issue." — Participant, MSM \& heterosexual mixed group Male participants' mistrust of the PrEP was further compounded by their fears about PrEP safety, particularly its side effects, and the participants' distrust in their provider's ability to be transparent about these safety concerns. For instance, some participants did not trust that their doctor would be open and honest about potential side effects associated with taking PrEP, to which they remarked, "Like liver damage, stuff like that, the doctors cannot answer those questions for me."

Participants' skepticisms about PrEP and mistrust of health care providers is born from mistreatment of African Americans in research as well as experiences of discrimination and disparities within the health care system. This unpleasant history, 
coupled with recent reports of continued experiences of differential treatment of African American individuals within the health care system, has caused many, especially men, in the community to have a distrust of biomedical interventions and health care systems.

\section{Stigma}

Participants endorsed stigma beliefs that impacted decision-making around PrEP use for HIV prevention. Stigma appeared to be expressed as internalized (participants' feelings of shame associated with society's judgmental perceptions of them using an HIV medicine like PrEP or as persons who are gay) and as externalized (participants' judgmental feelings and expressions about others perceived to be HIV positive or taking PrEP for HIV prevention). Participants reported on prevalence of HIV-related stigma within the African American community. These were expressed as accounts of the community views and treatment of persons with HIV or those who are perceived as being at a high risk for HIV, such as gay individuals.

Some participants also confessed that at one point they, too, endorsed such sentiments and even perpetuated stigma against persons suspected to be HIV positive. Here is a conversation participants exchanged about stigmatizing attitudes towards persons with HIV:

Participant 3:"I used to be scared of HIV I would never talk to nobody that had HIV. I would not be in a relationship with nobody... don't do that, don't kiss me, don't eat off of me."

Participant 2: "see that's how I am like it, it terrifies me."

Participant 4: “I was scared...don't don't touch me don't talk to me. Don't kiss me don't be near me don't don't do me nothing." 
Many participants felt that people would think badly of a person taking medication to prevent HIV and that person would potentially be targeted and judged or mistaken for being HIV positive. Thus, participants feared being stigmatized by others within the community if they were seen taking PrEP for HIV prevention.

"I think for me, just I think the stigma around it is that if somebody sees me taking the pill, that I already have HIV. And it's not to prevent it, but it's that I'm on retroviral treatment." - Participant, MSM + heterosexual mixed group "Yeah. Like people our age, people are gonna joke about it. Like, "Oh you taking the pill, you got HIV." — Participant, MSM \& heterosexual mixed group Some of these stigmas were born from the public's interpretation of the portrayal of gay individuals in PrEP advertisements. Participants expressed that the excessive targeting of PrEP towards gay individuals produced unintended consequences. They remarked that the practice served to stigmatize gay individuals and to shame or exclude others who may need PrEP but do not identify as gay, as such individuals might assume PrEP was intended only for the gay community.

"I think the whole label aspect of it all shames. Sometimes it demasculates some guys if they're put in a category, the same category with people like myself or like my friend. You know what I mean? They don't want to be labeled as gay men but they still need that $[\mathrm{PrEP}]$ for the same protection, and they need to know that it's not just subject to the gay community." - Participant, LGBTQ+ only group Consequently, participants expressed the need to decrease stigma associated with using PrEP for HIV prevention. They suggested that PrEP should be normalized; that is, 
more people should be made aware of PrEP in ways that emphasize its benefits and reduces misperceptions about its purpose.

"Actually target ... How about this? Normalize, like you normalize condoms and everything else. Normalize that [PrEP].” — Participant, LGBTQ+-only groups. "I wanna be optimistic and assume that maybe if we can normalize it [PrEP] and kinda take some of the taboo and the big bad away of it. 'Cause a lot of people don't know about it, so automatically people will assume, I see this dude taking a pill every day. And I probably would honestly wanna hide and take the pill. I'm gonna sneak off, pop this pill, and then come back. But I feel like if more people did it or more people knew about it, maybe they wouldn't think of it so bad." Participant, MSM \& heterosexual mixed group In addition to decreasing PrEP-related stigma, participants further expressed the need to decrease the HIV-related stigma in order to increase willingness to engage with PrEP for HIV prevention. They highlighted the impact of sexual silence within the community and implied that this practice was not only perpetuating stigmas but also indirectly impacting HIV risk within the community. They suggested the need for encouraging more open conversations about HIV, sex, and sexuality within their communities to normalize the subject and increase perceived risk of HIV and perceived need for prevention interventions like PrEP.

"It's going to be a long problem because of the stigma, until the stigma can be reduced no one's going to want to talk about it [HIV] and people are going to be like "Oh you think ..." Like how he said, because the stigma is there, it's not a cold or something that you know you can get help, it's a long-term thing, so like if you 
have it [HIV], it's going to affect your life and people will look at you different." - Participant, heterosexual mixed group "I agree with her, but the hardest thing is even if somebody was trying to talk to somebody about HIV, that's kind of a subject that a lot of people don't want to touch on, it even if I was offering it [PrEP] to you a lot of people get defensive and say "Oh, you think that I'm this type of person that catches a disease like this." So you've got to find a way to get out there in a way that's not directly seeming like an attack, just as a way of 'You can protect yourself by doing this.'

— Participant, heterosexual mixed group

\section{PrEP availability and accessibility}

Accessibility was described in terms of cost (lack of affordability, lack of insurance) and where to go to obtain PrEP if participants decided that they needed it.

\section{Cost}

Participants were interested in PrEP, but worried about the cost. Many were unaware that PrEP was covered by most insurance plans, and were also unaware that medication assistance programs are available to reduce the cost burden and reduce PrEP access barriers associated with cost of medication.

"I mean I guess PrEP is good and everything, don't get me wrong, and I've actually even tried it but I feel like... when I looked up information about PrEP afterwards, with PrEP there was something like it costs $\$ 4,000$ to get and like you said some insurance cover it, some insurances don't. I wasn't sure about which was which and it made me think ... Make it seem like it's easy to get but it isn't." — Participant, LGBTQ+-only group 
Overall, participants saw PrEP as welcome intervention and felt like people would be willing to obtain it as long as they could readily access it. They further suggested creating more PrEP awareness that explicitly shows where and how to easily access PrEP, including where to obtain it at no cost since some people may be poor and not have health insurance.

"And this was before I actually Figured out how to get it or that my insurance even took it. I think that making things more realistic and showing things, expressing what it actually is and then also showing how people can actually get the medication could even help." - Participant, LGBTQ+-only group "I feel like for something like this, and you got people who live in poor neighborhoods and they don't go to the doctors 'cause they don't have health insurance, you would have to let them know. You have to come to them and reach out in their communities to let them know, 'Look, this is what we're offering, it's free, come see us and we'll give it to you.' 'Cause they're not going to go find out about it." -Participant, female-only group

\section{Where to obtain PrEP and Provider preferences}

Some participants saw providers as facilitators to PrEP engagement and uptake while others perceived this as a barrier to PrEP education and uptake. Discourses among participants regarding reasons for lack of PrEP awareness prior to the study revealed that several participants, especially heterosexual women, expected to have heard about PrEP during their routine health care visits. They felt that their primary care provider (PCP) should have at least mentioned PrEP to them, but that did not happen. Participants expressed surprise and even anger for not having been told about PrEP by their provider. 
"I mean, I don't think anybody ... Like I've been to the doctor countless times and nobody has ever mentioned it." - Participant, heterosexual mixed (male and female group)

"But it sounds like none of, and I'm going to include myself in this too, none of our physicians, none of our primary care providers or doctors or nurses or anybody we talk to are mentioning this to us. I know that's been my case and none of y'all hadn't heard of it from your providers?" - Participant, heterosexual female-only group

"I would say I usually get my full STD testing from my nurse practitioner when I go to see her every year, and I have also gotten it from the city, and in neither of those experiences have they mentioned PrEP, which is interesting and maybe they don't ... I know I get a lot of questions, and maybe they don't think I'm at high-risk per se, but I never hear about it, and I try to do as much preventative testing as possible." - Participant, heterosexual female-only group

Women's reactions hinted at their positive relationship with health care providers. It appeared from their responses that African American women trust and even expect their health care provider to educate them about HIV prevention including PrEP. "My health care provider, I will say I'm comfortable with her. I've asked her questions about stuff that I didn't want to, but I know I needed to ask, and she didn't make me feel uncomfortable at all, she just answered my question and give me good advice. This is someone who [inaudible 00:35:42] give you a straightforward answer and not look at you like they're judging you." Participant, heterosexual female-only group 
Consequently, the participants expressed the desire to have PrEP readily available at provider locations such as STD and HIV testing clinics. They further suggested that HIV services including PrEP needed to be integrated with other STD testing services to normalize HIV, rather than keeping HIV separate and on a somewhat higher pedestal than other STDs, which can make HIV conversations seem more of a taboo, further perpetuating the stigma around HIV. One participant suggested:

"It could be a clinic of STD. It could be a STD clinic all it wants to be but I think that you know how they separate, well they separate pretty much. HIV is the only thing that's separate from almost everything else. If you have herpes, gonorrhea, all that, you still go to the same place, but if you have HIV, you are over here. If maybe they could move that since y'all controlling it and y'all got a pill for it and move it on over to the rest of the STDs." - Participant, LGBTQ+ mixed group In sharp contrast to heterosexual women, some male participants, especially MSM, did not feel comfortable learning about PrEP from a PCP. They perceived that the health care system's practice of targeting PrEP promotion towards gay individuals perpetuated stigma and stereotyping of gay individuals by health care personnel. For instance, one participant was highly displeased when they were handed a PrEP-promotion material like a pamphlet at a provider's office during a routine visit. This approach infuriated the participant who alluded to the tendency of the health care system to target gay persons for PrEP and HIV prevention. He perceived that the health care personnel were operating under the assumption that gay persons are at higher HIV risk and thus need to be made more aware of PrEP compared to heterosexual individuals. 
"Stuff like that, that's the people [the heterosexual individuals] who need it. We know about it because we're gay, they're gonna throw it in our face, we going to the doctor and the nurse gonna slip us a little pamphlet because she think we gay, bitch! I'm straight! I got okay husband and four kids, now what you wanna do? Then she feel stupid, she gonna slide you a pamphlet because you gay. It needs to be in the straight community, you know what I'm saying? There's a big bucket of condoms in the gay club, why aren't there a big bucket of condoms in the straight club? Because most of them niggas is going home with somebody too!"Participant LGBTQ+ mixed group

It appeared that male participants' mistrust of the health care system was born, in part, from unpleasant personal experiences and perceptions of unfair treatment at health care facilities, particularly at public/free clinics. Participants hinted at differential treatment at public clinics compared to private clinics. Thus, they indicated that if they could afford it, they preferred to access care at a private facility. One participant remarked:

"Because I'm able to work a good 9-5, I'm able to go to a private physician. I don't have to go where everybody else [goes]. I don't have to go to where they go at to get a STD check, I don't. I never have. When I went down there one time..., I was mortified. Never went back... I always just go to the doctor. Something's wrong, I just go there first thing in the morning." - Participant, MSM-only group Further, some MSM perceived being seen getting care from certain facilities as stigmatizing because these facilities are associated with HIV services for persons living with HIV within the community. 
"Certain times if you go certain places it just holds a certain stigma. [HIV clinic], if you go to [ASO location], if you are seen at any of those places then you got the bug and I think that's the box [separating HIV-related services] I'm talking about.”

— Participant, LGBTQ+-only group

Conversely, the negative experiences and intense displeasure with receiving sexual health services from health care providers expressed by gay individuals did not seem to be shared by the one transgender woman in one of the LGBTQ groups. This transgender woman expressed a positive inclination towards health care provider role in STD services and appeared to be in support of receiving care through the PCP. They said,

"For me when I go to see my Endocrinologist, I'm able to do blood work and I can do my STDs every couple months with that and I keep up with it and I have had issues but I only caught it because I was able to do that. That's something that is good for me, but I'm a transgender girl. I'm not a gay guy.” — Participant LGBTQ+-only group

This is an indication that, to build trust between the health care system and African Americans, the health care system would need to improve the quality of experiences so that the population can feel more comfortable accessing care at these facilities.

\section{Interventionists' PrEP engagement strategies}

Participants expressed responses to current AIDS service organizations' (ASOs) PrEP engagement strategies, participant preferences, and recommendations regarding what would facilitate PrEP engagement — how ASOs should interact with the 
community (Ayangeakaa et al., 2019). These were two-fold: (a) responses to current engagement practices (that is, efforts to engage with African American priority groups for PrEP uptake and other HIV prevention services), and (b) preferences and recommendations for future PrEP engagement strategies.

\section{Responses to current ASOs' PrEP engagement practices}

Participants expressed dissatisfaction with current practices of ASOs regarding outreach to African American communities. For instance, they indicated that ASO presence was not being felt within the African American community. Many were unaware of these organizations' existence and their efforts around PrEP within the community. This was an indication that current ASO efforts around PrEP promotion are not adequately reaching young African Americans who could benefit from PrEP. Specifically, participants were frustrated that service locations were out of reach of young African Americans, citing long commute times of one to two hours.

"Not even just downtown, but there's also places all the way over in east, towards the east end, Westport Rd., Lyndon Lane [areas of the city that are occupied by predominantly White individuals], areas like that, that have all these resources and yet these resources are only stuck in one area that's very difficult to get to, especially by bus that take practically an hour or two if you miss it. Why isn't it like ... If there was more places where they could be actually reachable to younger people then it would be a hell of a lot more easier to even take care of themselves, but they don't have any knowledge, they don't have any resources, they don't have places where they can go in these areas." - Participant, LGBTQ- only group 
"Why is there, honestly, why isn't there more of these HIV resources like even towards places like the west end [an area with a high concentration of African Americans]?" - Participant, LGBTQ+-only group

\section{Preferences and recommendations for future PrEP engagement strategies}

Preferred strategies for ASOs to better engage the African American community with PrEP promotion were suggested by participants. Specifically, they expressed preferences in the characteristics of the people who should provide PrEP to African Americans. Examples of suggested preferred personnel and qualities of persons providing outreach included peers, other trusted persons who look like participants (minority, specifically African American) and members of the community, similar age group, personable, professional, and knowledgeable (should be well versed in the concept of $\operatorname{PrEP})$.

Facilitator: For those of you who haven't heard about it, who would be the best messenger

"I think it should come from people in our age group, the same age group as us. 'Cause if it's coming from an older person they're not gonna pay attention. But if it's coming from people the same age as I am I mean ..." - Participant, MSM \& heterosexual men mixed group

Participants indicated they would be more likely to accept PrEP-related information if it were to come from or be recommended by a trusted person, typically someone who looks like the participant (same race), or at least someone with whom they could relate. Thus, some participants expressed strong sentiments against employing personnel of a different race to deliver PrEP messages to African Americans. Many 
participants did not feel like they could identify with someone who was not African American because the participant did not believe the non-African American personnel, especially if they were White, could understand the struggles of an African American person.

"I have to be honest with them. Please don't give me a White person. Please don't, 'cause they could never understand my struggle and what the hell I'm going through. I cannot talk to this White person.” — Participant, MSM-only group "I can't identify with him, because he's White and preppy. I can't identify with him because he's ... I can't even remotely identify. I don't want you coming to my house. Do not talk to me.” — Participant, MSM-only group

Despite their recommendation to hire PrEP outreach personnel from the African American community, participants cautioned against ASOs hiring someone simply because they fit the recommended preferred characteristics. Participants expected the hired African American personnel to be qualified for the position - be knowledgeable, as well as act professionally.

"Most definitely. I mean to me I feel like I can smell that, but sometimes some people are hired off their credentials, which is very sad because you will get ... For me I would rather see a White professional not pay me attention and do their job than a black person sit in front of me and not be professional and not do their job, or the two. If I see a black person doing their job and then they're not attentive to me it would probably would make me more upset, just me personally. Just because I feel like you work this hard to get here, you should at least like what you're doing. - Participant, LGBTQ+-only group 
"For me, somebody who's taking it or knows someone who's taking it." -

Participant, female-only group

"More knowledgeable about it." — Participant, female-only group

Participants also provided recommendations for PrEP promotion as they felt the current promotional efforts were not resonating well with African American youth. They thought, for instance, that the current PrEP commercials overly focused on sex and only seemed to cater mainly to Whites. So, participants did not feel like they could relate to the current PrEP promotional efforts. They expressed a desire to see the commercials modified to diversify the modes of transmission as well as feature more African American persons.

"I think if they advertise it, not just through sex as well, because that commercial is like, it's very, sex oriented and you can get HIV in various different forms, ways, whatever. Oh, it was White too..., just the commercial alone, that I've seen on YouTube, it was White. And that's another thing too, like, it was White. I mean, that's just the end of the sentence. It was White.” — Participant, femaleonly group "I [will] look at it like if they mixed it, you know, threw some of us [African Americans] in there because it is more, you know, so I can understand that." Participant, female-only group

Participants further suggested the need to make PrEP publicity and education around sexual health more inclusive of various priority groups (PWIDs, MSM, LGBTQ, heterosexual persons, etc.) so that everyone, regardless of their sexuality, especially 
heterosexual individuals, would view themselves as being at risk and recognize their need for PrEP.

"This needs to be the conversation everybody's having, not just women and not just gay men. Like, straight men that are having sex with a lot of women." Participant, female-only group

"When I see the one PrEP pill commercial that I do see, I see two transgender girls which pretty much knocks the whole thing down really. Like when you catch that it's like "oh shit, it's not for me," so I think the trans girls pretty much know about it because they pretty much know everything. I think that it [the commercial] should be comfortable enough for straight men to really get into because then I feel like women would probably follow suit.”- Participant, MSM $\&$ heterosexual male mixed group "Also, it's [HIV] something that people are scared of, so I think the trans woman being the voice of PrEP is wrong. I think it should be endorsed by a heterosexual man...like Odell Beckham or some sh*t... And then I think a lot of more people will feel comfortable...So, I feel like the message needs to be spread, the young people need to know for the future, but if we want to survive as a whole, we all need to update our education on sex because what it was five years ago and what it was ten years ago, it is not today..."- Participant ,LGBTQ+ \& heterosexual male mixed group

Furthermore, participants recommended that PrEP awareness and education should include statistics on HIV rates and PrEP eligibility for African Americans in various risk groups (not targeting only one risk group, like MSM). 
"I think putting the statistics out there, but I think just doing it mindfully, I guess. Because I don't think we just wanna make it seem like oh, here's Black people, black gay people, they got HIV. Because I think that's what a lot of the stereotype and a lot of the conversation. So, I definitely don't ... 'Cause then it just feels like a loss of hope."-Participant, MSM \& heterosexual male mixed group "Some statistic about heterosexual women having it..."- Participant, femaleonly group

Additionally, participants expressed their preferences and offered suggestions for PrEP promotional campaigns to increase PrEP awareness within their community. They felt multimedia campaigns (posters, billboards, social media etc.) would be more effective and wanted these efforts to be transparent in presenting the information, include side effects, and use colorful visuals and slogans.

"Posters, billboards, that could work out too. 'Cause throughout our campus we have little signs sticking out the grass, so then free HIV testing and stuff like that. That's very beneficial as well." - Participant, MSM \& heterosexual male mixed group.

"Put them on billboards. I read billboards anytime." - Participant, heterosexual mixed group.

“Ads, like on YouTube. Some of them ads you just can't skip, you have no choice but to watch them." - Participant, MSM \& heterosexual male mixed group.

"To keep it 100, I think I would just be G off the muscle with the side effects, because, that's the first raise of question, then the second question is going to be how much. So that's off top." - Participant, heterosexual male-only group. 
More importantly, participants suggested that ASOs should consider situating their offices in places that are easily accessible to young African Americans to increase likelihood of engagement. They wanted ASOs to reduce current access barriers by placing more services within the reach of many young African Americans such as making PrEP services available and accessible at neighborhood clinics and incorporating PrEP into routine care such as STD services.

"I would say go setup shop down in the west [an area with a high concentration of African Americans], go ahead and fill that with the black professional..." Participant, LGBTQ+-only group

Overall, participants wanted to see more ASO engagement within the African American community. They believed ASOs could better establish their presence within the African American community by building rapport through hiring of community members who identify with young African Americans and by that having services within reach of the community. This was expected to improve engagement with PrEP services and ultimately PrEP uptake.

\section{Discussion}

The purpose of this study was to explore and understand multi-level barriers and facilitators to PrEP engagement and uptake among multiple African American priority groups of heterosexual men and women, in addition to LGBTQ+ individuals. Understanding reasons behind reticence to PrEP use and engagement among African Americans in various priority groups is a necessary step towards developing effective measures to reduce disparities in PrEP use among them. Findings revealed multi-level influences on PrEP use including, interpersonal factors (opinions of referents), 
sociocultural factors (stigma, medical mistrust and conspiracy beliefs), and structural issues (cost and availability of PrEP services).

Opinions of referents influenced PrEP-use intentions for many participants in the study. For instance, participants believed they would be more likely to use PrEP if PrEP was widely accepted and endorsed as an HIV prevention option by trusted persons within the African American community. Consequently, many participants cared about the opinions of their referents. They were unwilling to engage with or consider using PrEP if they perceived that they would be judged by their peers for taking a pill to prevent HIV and vice versa. This finding is indicative of the potential for social networks to impact PrEP acceptability and engagement among African American youth since youth are likely to trust the opinions of their peers. If trusted individuals within these networks were to endorse PrEP, chances are others would follow suit. This finding further reinforces evidence suggesting that injunctive norms (perceptions of who approves or disapproves of a behavior, in this case PrEP use) have an impact on performing any given behavior (Morris, Hong, Chiu, \& Liu, 2015; Schnarrs et al., 2018). Thus, understanding the perceived norms of PrEP among African American priority groups is critical to scaling up PrEP uptake among them.

Similarly, fear of being stigmatized for using PrEP was expressed by most participants in the study who endorsed beliefs that people within their community would think badly of a person taking a pill associated with HIV (PrEP). Others feared being mistaken for being infected with HIV if they were seen using PrEP. Participant discourses also indicated that PrEP publicity was partly to blame for perpetuation of homophobic ideologies, PrEP-related stigma, and stigma associated with being gay. 
Specifically, due to excessively targeting of PrEP advertisements towards gay

individuals, heterosexual individuals in the study erroneously assumed that PrEP was not for them, but only intended for LGBTQ+ populations. As a result, heterosexual individuals associated PrEP with gay persons and thus did not want to be seen taking PrEP nor did they see themselves as being at risk for HIV. This behavior is in concert with evidence suggesting that if individuals do not associate with certain characteristics of the persons to whom the behavior is linked, they will not engage in the behavior (Schnarrs et al., 2018). This finding highlights the impact of perceived social norms and their potential for influencing PrEP engagement and willingness to use PrEP. Research on social norms further shows that performance of behavior is influenced by who the potential performer perceives is linked to that behavior (Andrew et al., 2016; Vissman et al., 2011). Addressing the unintended consequences of associating PrEP with LGBTQ+ individuals in future PrEP publicity is important to reduce homophobia, homonegativity, and stigma that have previously been shown to negatively impact HIV risk and PrEP uptake among African Americans (Arnold, Rebchook, \& Kegeles, 2014; Elopre et al., 2018). Interventionists should honor participant's suggestions to design more inclusive PrEP publicity that equally represents all demographics of persons demonstrating heightened HIV risk, not just LGBTQ+ individuals.

Another significant finding in our study was medical mistrust, and it was observed to be reinforced by conspiracy beliefs. Mistrust of PrEP resulting from the mistrust of the health care system, along with the endorsement of conspiracy beliefs, were prevalent in the study. Other studies also demonstrated that deeply expressed concerns about stigma, endorsement of conspiracy beliefs, and expressed medical 
mistrust by African American priority groups had considerable impact on PrEP-use intentions among this population (Eaton, Driffin, Kegler, et al., 2015; Eaton et al., 2014; Eaton, Kalichman, et al., 2017; Elopre et al., 2018; Lelutiu-Weinberger \& Golub, 2016). Medical mistrust in our study was more apparent among male participants. It is plausible that this prevalence of mistrust among male participants is due to the lingering effects of research improprieties like the Tuskegee study, that mainly focused on African American males. This historical unethical treatment of African American men has been etched on the minds of the African American community and continues to impact interactions with the health system today. Evidence further reveals that African American men continue to experience unfair treatment and discrimination in the current society, and particularly within the health care system (Scharff et al., 2010; Underhill et al., 2015). The health care industry will need to consciously assess and address factors that perpetuate distrust among African Americans to improve health care access among African Americans, especially males.

Additionally, structural factors such as cost, availability, and accessibility of PrEP were discussed by participants and shown to influence their decisions to engagement with PrEP. Participants demonstrated interest in PrEP but were concerned about affordability. Concerns about cost, however, were born out of a lack of knowledge that PrEP is covered by most insurance and medication assistance programs also exist to help uninsured participants interested in PrEP to access it at no monetary cost to them. This underscores the need for awareness campaigns and outreach efforts geared towards African Americans to highlight information pertaining to PrEP access such as coverage by most insurance companies as well as locations where PrEP is readily available. Also, with 
regards to access, PrEP cost and accessibility, in terms of provider preferences, were among the main concerns expressed. Determinants of access within the environments of the target population would need to be taken into consideration if PrEP intervention is to be effective; otherwise, the efforts are pointless (Richardson, 2014).

Furthermore, provider preferences and expectations appeared to shape PrEP engagement and willingness to use PrEP. For instance, some of the women in our study blamed their health care providers for never mentioning nor recommending PrEP during routine health check visits. This was an indication that these women expected their PCPs to be knowledgeable about PrEP and would be likely to trust PrEP information coming from their provider. This could have positive implications for PrEP implementation, especially in primary care settings frequented by African American women. In previous studies, African American women indicated that they trusted their primary care providers to provide PrEP services and indicated willingness to use PrEP if a provider recommended it (Auerbach et al., 2015; Wingood, Dunkle, et al., 2013). However, our findings along with evidence from previous research revealed that women as well as their providers have limited PrEP knowledge (Seidman, Carlson, Weber, Witt, \& Kelly, 2016). Consequently, the failure of health care providers to adequately provide PrEP information to or address PrEP needs of African Americans, especially women, has been previously documented (Collier et al., 2017; Elopre et al., 2017) and is worrisome. This underscores the need to equip providers catering to women such as those in reproductive health care settings to orient and educate their female clients about HIV risk and PrEP. Increasing PrEP awareness among women holds potential for increasing PrEP uptake among women and thus decreasing HIV risk (Flash et al., 2017) especially since African American 
women are the group with the second highest risk of HIV after MSM and bisexual men of all races (Centers for Disease Control and Prevention, 2018f).

In contrast to heterosexual female participants, MSM and other males in our study were not positively inclined towards receiving PrEP education from their PCP. PrEP promotion at health care provider locations was perceived as stigmatizing and stereotyping to MSM. For instance, some men felt insulted when handed a flyer about PrEP during a visit to the health care provider. This has implications for PrEP engagement and access among African American men, especially MSM. Other studies have documented that African American men do not feel comfortable discussing or disclosing sexual life with their providers (Lelutiu-Weinberger \& Golub, 2016; Underhill et al., 2015). African American men's discomfort with disclosing sexual health to providers stems from medical mistrust and perceived discrimination (Underhill et al., 2015). MSM, however, along with other participants, seemed comfortable engaging with community ASOs if the ASO personnel possessed characteristics with which African Americans identified, such as other African Americans and if the personnel were knowledgeable and professional. This calls for additional outreach strategies tailored towards African American communities that address the needs of the community.

Finally, participants were unsatisfied with current ASO outreach strategies for engaging African Americans with PrEP. Many did not feel that ASOs were present enough within the community. They also did not perceive that PrEP outreach efforts resonated well with African American high-risk groups. They wanted to have HIV prevention and PrEP promotional messages be more inclusive of various risk categories and tailored more towards African Americans. Additionally, participants recommended 
PrEP integration into existing sexual health services offered at accessible locations within their neighborhoods for ease of access. Moreover, participants indicated strong sentiments and desired to see more African American people or peers of high-risk groups employed by ASOs to conduct HIV/PrEP outreach within the African American community. They believed this practice would make ASOs more relatable and thus improve engagement with PrEP services and PrEP uptake among young African Americans.

\section{Conclusion}

Findings highlighted focus areas for tailoring outreach to African American priority high-risk groups for improving PrEP engagement, uptake, and outreach. Sociocultural factors (stigma, medical mistrust, and conspiracy beliefs), along with structural issues (cost and availability of services) may act as barriers or facilitators to PrEP engagement and uptake among African Americans. Health promotion interventionists should take advantage of this knowledge to tailor interventions that address barriers and maximize facilitators to PrEP engagement among African American priority groups. AIDS services organizations should hire more African American individuals from the local communities who can draw upon insider knowledge about their community to help ASOs better establish their presence within the community and scale up PrEP efforts. Future research should consider working directly with the communities to co-develop strategies for decreasing stigma, medical mistrust, and conspiracy beliefs among young African Americans in ways that do not stigmatize any segment of the population but rather strive to normalize PrEP as well as increase perceptions of HIV risk and need for PrEP among priority groups 
CHAPTER VI PAPER 3

“BECOMING ONE WITH THE COMMUNITY”: A GROUNDED
THEORY STUDY EXPLORING AIDS SERVICE ORGANIZATIONS'
STRATEGIES FOR SUCCESSFULLY IMPLEMENTING HIV PRE-
EXPOSURE PROPHYLAXIS (PREP) OUTREACH AMONG AFRICAN

AMERICANS

\section{Introduction}

Persistent disproportionate rates of HIV among African Americans, coupled with pervasive disparities in uptake of high potency biomedical interventions, cast a dark shadow on prospects of ending the HIV epidemic soon, unless drastic measures are taken. Despite only comprising $13 \%$ of the U.S. population, African Americans reportedly account for the majority (42\%) of all new of HIV diagnoses in the U.S. annually (Centers for Disease Control and Prevention, 2019b). The CDC recommends the use of preexposure prophylaxis (PrEP), an FDA-approved, once-daily oral antiretroviral medication for decreasing incidence in HIV-negative individuals with heightened HIV vulnerability: MSM, persons who inject drugs, and heterosexual individuals demonstrating high-risk sexual behaviors - sex without condoms or with a partner of unknown HIV status (Centers for Disease Control and Prevention, 20181). PrEP is highly effective in decreasing HIV incidence in various high-risk groups when used as recommended (Baeten et al., 2012; Choopanya et al., 2013; Grant et al., 2010; McCormack, Dunn, 
Desai, Dolling, Gafos, Gilson, Sullivan, Clarke, Reeves, Schembri, et al., 2016; Sheth et al., 2016). Yet, uptake is marginal among populations like African Americans, women, and youth who stand to benefit the most from PrEP (Bush et al., 2015; Kuhns et al., 2017; Siegler, Bratcher, et al., 2018). Disparities in PrEP uptake are especially present among African Americans as evidence depicts PrEP prescribed to and used by fewer African American priority groups compared to Whites (Bush et al., 2015).

Research demonstrates a plethora of challenges to PrEP uptake among African Americans. These include low PrEP knowledge, awareness, poor attitudes and perceptions, and low perceived HIV risk and need for PrEP (Bauermeister et al., 2013; Collier et al., 2017; Eaton, Driffin, Bauermeister, et al., 2015; Elopre et al., 2018; Smith et al., 2012). Additionally, access, availability, stigma, conspiracy beliefs, medical mistrust, and provider preferences have been shown to preclude PrEP engagement among African American groups (Eaton et al., 2014; Eaton, Kalichman, et al., 2017; Elopre et al., 2017; Lelutiu-Weinberger \& Golub, 2016; Wingood, Dunkle, et al., 2013). While PrEP holds the potential to slow down and even end the HIV epidemic, this goal can only be achieved with optimal engagement and uptake in populations with the highest HIV vulnerability (Richardson, 2014).

AIDS service organizations (ASOs), who include community-based organizations (CBOs) and other entities like local clinics and community health centers providing HIV testing and other STD services, hold the potential to engage vulnerable populations with PrEP for HIV prevention (Collier et al., 2017; Elopre et al., 2017). Receiving services such as HIV testing, routine doctor's visits, and condoms from a CBO have been previously linked to PrEP awareness (Raifman et al., 2017). Evidence further suggests 
that these community organizations are strategically positioned to reach and engage vulnerable individuals who would otherwise not have access to those services through traditional methods like clinics or hospitals (Flash et al., 2017). Thus, CBOs could integrate PrEP into existing programs to improve scale-up efforts (Wingood, Rubtsova, et al., 2013).

Despite this recognition of ASO's important role in HIV prevention, evidence suggests that few CBOs are adequately engaging populations at highest HIV vulnerability with PrEP or may be ill equipped to do so effectively (Elopre et al., 2017; Smith et al., 2016). Far more worrisome is evidence suggesting that ASOs are barely reaching and engaging populations with the highest HIV vulnerability, specifically African American priority groups like MSM, women, and youth (Ayangeakaa et al., 2019; Elopre et al., 2017). A study of ASOs implementing PrEP within a southern U.S. urban locale revealed that limited accesses to African American communities as well as limited agency capacity, specifically staffing issues, were among several challenges to effectively reaching and conducting outreach with African American priority populations (Ayangeakaa et al., 2020). Incidentally, according to study results from an assessment of young 18-29 years old) African American priority groups in the same urban southern U.S. city as mentioned above, participants reported that ASOs' presence was not being felt within their community; participants also indicated the need for more African American personnel or peers of high-risk groups employed by ASOs to conduct HIV/PrEP outreach within the African American community (Ayangeakaa et al., 2019). This is an indication that ASOs may be employing status quo approaches to PrEP engagement and outreach that are not suitable for nor preferred by minority populations, 
such as African Americans, who may require culturally tailored approaches to engage with health care-related interventions like PrEP.

Research evidence on ASO struggles underscores the importance of having proven strategies or a guide for ASOs to tailor PrEP engagement and outreach to African Americans to scale up PrEP uptake among them. Currently, proven strategies for ASOs to successfully scale up PrEP uptake by effectively conducting PrEP outreach with hardto-reach African American groups at heightened risk of HIV either remain understudied or are sparsely published in the literature. To fill this knowledge gap, this study utilized constructivist grounded theory (CGT) (Charmaz, 2014) methods rooted in symbolic interactionism (SI) (Blumer, 1986) and pragmatism (Lewis, 1976) to explore the meanings ASOs ascribe to PrEP implementation and processes (specific activities or actions) involved in successfully conducting outreach with African American groups at increased vulnerability to HIV. The objective was to develop a context specific framework grounded in experiences of ASOs of how they have successfully implemented PrEP outreach among African American priority high-risk groups. Findings are expected to inform the outreach efforts of other ASOs looking to improve outreach efforts with African American priority groups.

\section{Methods}

\section{Methodology, Philosophical Assumptions, and Interpretive Framework}

This study utilized a theory-methods package — constructivist grounded theory CGT method (Charmaz, 2014), which has its philosophical underpinnings in symbolic interactionism (SI) (Blumer, 1986) and pragmatism (Lewis, 1976) and assumes a strong constructivist worldview (Charmaz, 2014; Creswell, 2013). SI assumes that meanings 
and actions are formed and shaped by language and symbols, and there is reciprocal relationship between actions and meaning (Blumer, 1986; Charmaz, 2014). That is, people act towards any given situation based on the how they interpret it, and those meanings may, in turn, be modified by the situation (experiences and encounters) (Blumer, 1986; Charmaz, 2014).

The study was designed using CGT approach and through the lens of social constructivism from conceptualization to dissemination of findings. Constructivists believe that multiple realities and interpretations exist and are socially constructed through the lived experiences of individuals and their interactions with others (Creswell, 2013; Merriam \& Tisdell, 2015; Salazar et al., 2015). In this worldview, meaning is coconstructed between the researcher and the research participant and ideas are emergent (Charmaz, 2014; Creswell, 2013); that is, individuals (researcher and participants alike) have varying interpretations of the studied world and create meanings through interaction and sharing of various viewpoints (Charmaz, 2014; Creswell, 2013).

Thus, this study assumed that ASOs will approach PrEP implementation based on the meanings they ascribe to it, and their interpretations of PrEP implementation may be impacted by their unique experiences engaging with and providing PrEP outreach to African American priority groups. This also helped to determine the range of meanings of PrEP interpretation held by various participants and the corresponding actions (strategies) that were informed by those meanings. The research data derived from this type of approach forms the basis for context-specific frameworks for explaining processes embedded within these social interactions. 


\section{Recruitment, Sampling, and Data Collection}

\section{Recruitment and Sampling}

Participants were recruited via a (1) rigorous internet search of ASOs across the U.S., beginning with a list of CDC-funded CBOs (delivering HIV prevention services among populations with the greatest need) found on the CDC website and then expanding search criteria to include any ASOs in specific target cities fitting the set eligibility criteria (Table 1); (2) referrals from other ASOs; and (3) academic/researcher collaborators with ties to ASOs. Emphasis was placed on these cities/states: California, New York, Atlanta, Chicago, Philadelphia, Maryland, and Washington DC that have elevated HIV rates and have several ASOs and CBOs with established HIV prevention infrastructure that includes PrEP delivery and outreach.

Ten key informants (representatives knowledgeable about organizational PrEP practices) were selected from unique ASOs in six urban sites across the U.S. (Table 4) using purposive sampling (Cleary et al., 2014; Etikan, 2016). The selection was based on the following inclusion criteria: (1) have an established and robust infrastructure for PrEP service delivery and outreach; 2) have a proven track record of establishing and implementing successful PrEP-focused initiatives, particularly those that have done this with African American communities; 3) currently engage in HIV service delivery, specifically, PrEP outreach to the various African American high-risk study target groups; 4) are involved in PrEP activities for at least 1 year ;5) demonstrate client uptake; and 6) are engaged in PrEP education. Successful ASOs had established PrEP-focused initiatives and demonstrated a) sustainability, b) robust client uptake, c) established procedures and protocols, and d) effective PrEP education and outreach activities. 
University of Louisville, KY Institutional Review Board (IRB) approved study protocols. Participants were screened through a multi-phased screening process to ensure that they fit the inclusion criteria. First, the original list of ASOs came from a list of CDC-funded organizations providing HIV services across the U.S. in locales demonstrating highest HIV rates. These ASOs were already subjected to a rigorous federal grant funding application process that emphasizes demonstrated client uptake. Second, the ASO websites were visited to ascertain if their listed HIV prevention services included PrEP services (especially outreach). Third, ASOs were called or emailed to further verify if they fit the inclusion criteria. Those who did not (e.g. who did not conduct outreach to African American priority groups) were not allowed to participate in the study. Finally, ASOs were asked about client uptake, and those who reported demonstrated client uptake (measured by tracking their PrEP outreach and delivery efforts like number of PrEP referrals and/or PrEP prescription initiations) as a result of PrEP outreach with clients were invited to participate in the study.

\section{Data Collection}

Two rounds of semi-structured phone interviews were conducted with a sample $(\mathrm{N}=10)$ from October 2019 to March 2020. First round of interviews were conducted with participants $((\mathrm{N}=10)$ and second round interviews were conducted with some of the participants $(\mathrm{N}=6)$ from the original sample to fulfil theoretical sampling/saturation and member checking (Charmaz, 2014). Before commencing any aspects of data collection, a preamble consent was administered to all study participants. Participants were informed that their responses were anonymous and confidentiality would be maintained. Interviews were audio recorded with the permission of the participants. First round 
interviews ranged from 29 to 70 minutes. Second round interviews ranged from 13 to 59

minutes. Interviews were not incentivized. Interviews were audio recorded and lasted approximately 45 minutes. Interview audio recordings were transcribed verbatim by a transcription service (Rev.com). Identifying information was removed and transcripts were coded using Dedoose qualitative analysis software (Dedoose.com).

Table 1 National ASO Sample Study Eligibility Criteria

\begin{tabular}{|c|c|}
\hline \multicolumn{2}{|c|}{ National ASO Sample Study Eligibility criteria } \\
\hline ASO Inclusion Criteria & Exclusion Criteria \\
\hline $\begin{array}{l}\text { - Have an established and robust } \\
\text { infrastructure for PrEP service } \\
\text { delivery and outreach }\end{array}$ & $\begin{array}{l}\text { Does not have an established and } \\
\text { robust infrastructure for service } \\
\text { delivery and outreach }\end{array}$ \\
\hline $\begin{array}{l}\text { Have a proven track record of } \\
\text { establishing and implementing } \\
\text { successful PrEP-focused } \\
\text { initiatives, particularly those that } \\
\text { have done this with African } \\
\text { American communities }\end{array}$ & $\begin{array}{l}\text { Does not have an established track } \\
\text { record of PrEP outreach to priority } \\
\text { groups }\end{array}$ \\
\hline $\begin{array}{l}\text { Currently engage in HIV service } \\
\text { delivery, specifically, PrEP } \\
\text { outreach to the various African } \\
\text { American high-risk study target } \\
\text { groups }\end{array}$ & $\begin{array}{l}\text { Does not have established PrEP- } \\
\text { focused initiatives and does not } \\
\text { demonstrate a) sustainability b) } \\
\text { robust client uptake c) established } \\
\text { procedures and protocols d) } \\
\text { effective PrEP education outreach } \\
\text { activities }\end{array}$ \\
\hline $\begin{array}{l}\text { - Are involved in PrEP activities for } \\
\text { at least } 1 \text { year }\end{array}$ & $\begin{array}{l}\text { - Is not involved in PrEP activities } \\
\text { for at least } 1 \text { year }\end{array}$ \\
\hline - Demonstrate client uptake & $\begin{array}{l}\text { - Does not demonstrate client } \\
\text { uptake }\end{array}$ \\
\hline - Are engaged in PrEP education & - Is not engaged in PrEP education \\
\hline
\end{tabular}




\section{Data Analysis}

CGT analytic principles (line-by-line coding, focused coding, theory building, and memo writing) were employed for this analysis (Figure 10) and aided in data synthesis to allow themes and theories to emerge from the data (Charmaz, 2014). Part of the data $(\mathrm{N}=7)$ was initially coded line-by-line by two coders (SA and JS) using gerunds ("ing" words denoting actions). Initial codes were clustered to form 24 focused codes that were further refined into the final codebook with 18 codes with definitions using the most frequent and significant focused codes (Charmaz, 2014). The codebook was uploaded into Dedoose, a web-based data analysis software that organizes and facilitates coding (Dedoose.com). Two team members (SA and JS) independently applied the final codebook to part of the data ( $\mathrm{N}=4$ and $\mathrm{N}=3$, respectively) during focused coding to ensure consistency in code application and to increase credibility of the process. A pooled kappa score of 0.92 indicated excellent inter-rater agreement among coders (Cohen, 1960) during the first attempt. Following a discussion among coders, a 100\% agreement was reached with the second kappa test attempt. The final codebook was applied to code the rest of the data $(\mathrm{N}=10)$ during a second round of analysis.

At the axial level of analysis, two researchers (SA and JS) worked together to compare focused codes and discuss emerging categories and relationships among categories. Code matrices enabled comparison across and within cases to tease out nuances and variations and to create descriptive categories and subcategories from the data. This also ensured intercoder consistency between coders as $100 \%$ agreement was reached regarding finalized categories and sub-categories within each of the themes (Charmaz, 2014; Strauss \& Corbin, 1994). 
Memos were written throughout the research process such as during data collection and all stages of the analysis to develop and check emerging ideas (Charmaz, 2014). Through a series of successive analytic memos, a provisional category was developed — "becoming one with the community" - that comprised these codes: "meeting people where they are" that encompassed identifying gate keepers; building rapport; gaining trust; being present in the community; knowing your client; reflecting your client; hiring from community; and catering to other needs besides sexual health. Through successive sorting of comprehensive analytic memos and diagramming, coupled with across and within-case data comparisons, an agreement was reached between the two researchers that a preliminary process was developing and the decision was made to elevate the provisional category "becoming one with the community" to a final abstract category. Researchers further determined this category to be a process with several phases, encompassing multiple subcategories that existed within distinct phases accompanied with unique tasks. 
Figure 10 National ASO Key Informant Interviews Data Analysis process

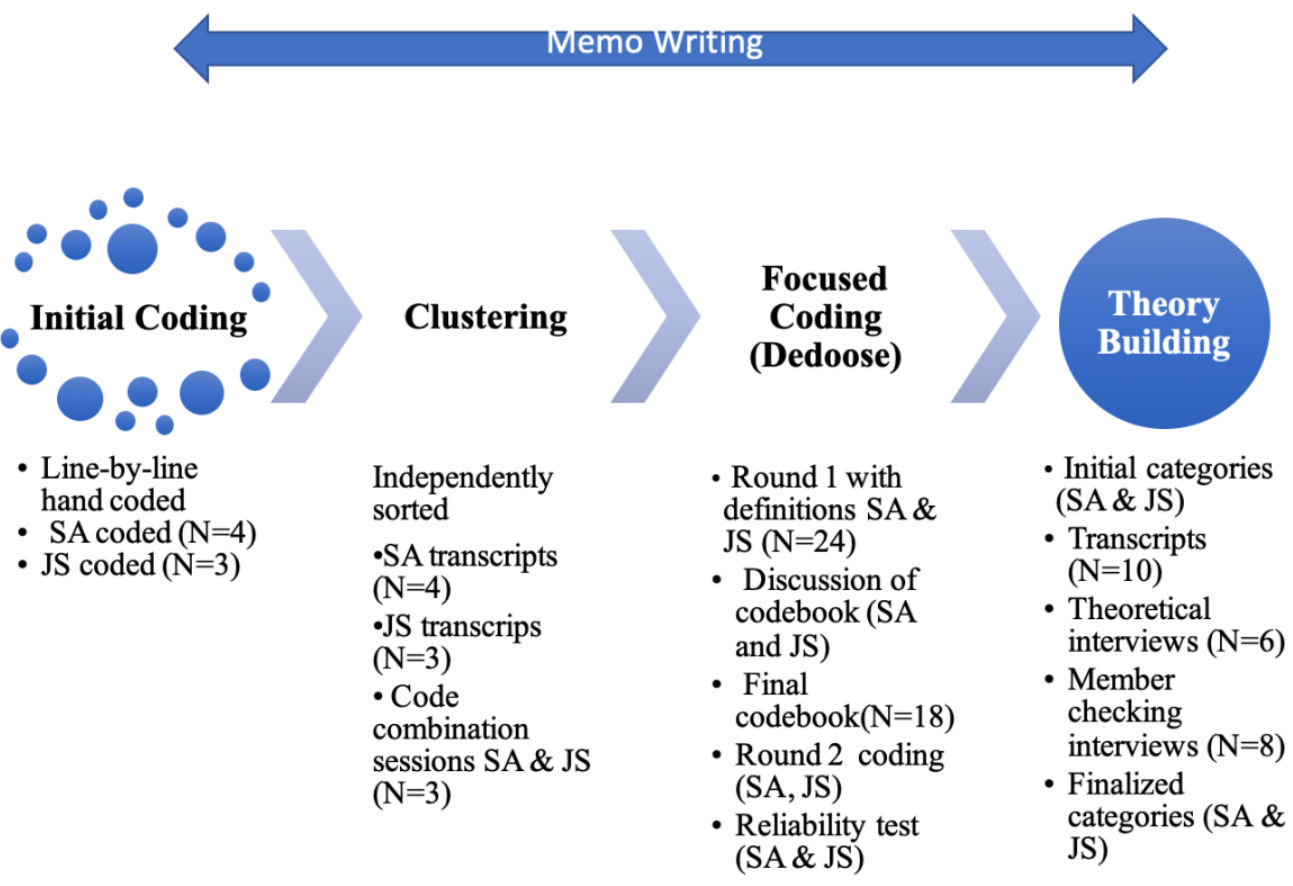

\section{Theoretical Sampling and Theoretical Saturation}

Following the data analysis and once a provisional category was developed and a process was conceptualized to form the context specific framework for PrEP outreach among African Americans, some areas were determined to need further explication. That is, there were a few gaps and missing links in the process that needed to be explored. For example, (1) the researcher needed to verify if the process was linear or dynamic, (2) verify conditions and connections for transitioning between phases, (3) verify temporal elements and sequencing of tasks associated with phases, and (4) explicate dimensions within some subcategories (Table 5). For instance, in phase III, for the subcategory "maximizing gatekeeper/ally connections," I needed to know: how are they selected, how are their skepticisms addressed, what is the temporal element between gatekeepers/allies and community access? What is the alternative strategy if an ASO cannot match 
personnel to population characteristic? Thus, theoretical sampling was necessary to further explicate and saturate all categories and subcategories within the emerging framework (Charmaz, 2014; Creswell, 2013; Glaser \& Strauss, 1967; Strauss \& Corbin, 1994). Saturation is defined as "the point in data collection when no additional issues or insights emerge from data and all relevant conceptual categories have been identified, explored, and exhausted" (Hennink et al., 2017, p. 592). Additional interviews ( $\mathrm{N}=6$, $\mathrm{N}=8$, respectively) were conducted with some participants from the original sample for theoretical saturation and for member checking (Charmaz, 2014). ASO key informants verified phases and confirmed that framework was a dynamic and "ongoing" process, rather than a linear and static one. They also provided missing links such as connections and conditions for transitioning between phases as well as sequencing of tasks and temporal elements associated with various phases and sub-categories and via a second round of interviews.

Researchers explored gaps or missing links in the process and conducted theoretical sampling to further saturate the process framework. Data collection and analysis were concurrent and sampling continued purposefully until all concepts of the developing theory were satisfied (Charmaz, 2014). Theoretical saturation was reached with 16 interviews: 10 first round (initial) interviews and six second round (repeat) interviews. The sample for the study was determined to be adequate for the study analysis as theoretical saturation was reached. This conclusion was made using a process of code saturation (the point at which all codes are accounted for to make the codebook stable) as well as meaning saturation "defined as the point when we fully understand issues, and when no further dimensions, nuances, or insights of issues can be found" 
(Hennink et al., 2017, p. 594). Code saturation occurred by the seventh interview and meaning saturation occurred by the eleventh interview.

\section{Results}

\section{Sample Characteristics}

The majority (80\%) of the participants were African American and 20\% were Hispanic (Table 4). Six self-identified as male, two identified as female, and two as nongender conforming. One ASO had been providing sexual health (including HIV) services for more than 30+ years, four ASOs for 20-30 years, two of them for 10-20 years, and one for less than 10 years. Duration of PrEP services also varies with one ASO providing PrEP for more than 6 years, five of them for 3-5 years, and four of them for less than three years. Although all $(\mathrm{N}=10)$ ASOs offered services to all demographics, $60 \%$ of them had MSM and transgender as their primary target population, $30 \%$ indicated serving persons who inject drugs (PWIDs), 20\% did not have a specific target group and $10 \%$ indicated heterosexual males as their primary target group (percentages do not add up to 100 since there was an overlap due to the fact that some ASO serving PWIDs also had other primary target groups).

\section{Context specific framework for ASO PrEP outreach with African Americans}

Participants described strategies and articulated processes involved in successfully conducting outreach with African American priority groups. Their responses indicated an abstract dynamic process, "becoming one with the community," involving various phases and stages as well as conditions for achieving success with PrEP engagement and outreach among African Americans (Table 5). 
Participants articulated the process of "becoming one with the community" as a major precursor to successfully implementing PrEP outreach within African American communities (Figure 13). This context specific framework for PrEP outreach was grounded in the voices and lived experiences of participants. The process involved three phases, each with corresponding tasks: (1) Grappling with pushback and challenges, (2) transforming challenges into opportunities, and (3) establishing authentic presence within the community. It should be noted that phases are not necessarily rigid. There is some overlap between phases.

\section{Phase I: Grappling with Pushback/Challenges}

For participants, the process of successful outreach with African Americans begins with an initial period of experiencing pushback and multiple challenges while attempting to implement PrEP engagement and outreach among African American groups. During this phase, participants recognize that if they are to succeed in their endeavors with African American clients, they would need to have meaningful interactions with their clients - as one participant put it, "get to know your client." This phase entails engaging in the tasks of acknowledging, assessing, and understanding the various types and origins of pushback and challenges. Thus, phase I required interacting with clients to understand the interplay of multiple factors that preclude client PrEP engagement and outreach among African Americans. This was a necessary first step in the process of "becoming one with the community." This phase served as a starting point for ASOs to begin assessing and understanding various types of issues (their corresponding root causes) that preclude PrEP engagement and uptake among clients. 
Table 4 Characteristics of key informants from national sample of ASOs. Sample size $(\mathrm{N}=10)$

$\mathrm{N} \%$

\section{Key informant characteristics}

Male

6

Female

Non-gender conforming

African American

Hispanic

Agency characteristics

Years providing HIV services

$30+$ years

20-30

10-20

Less than 10 years

Years providing PrEP services

$1-3$

$3-5$

$6+$

20

210

$8 \quad 80$

220

1210

5

220

220

$4 \quad 40$

$5-50$

110

Location (City/State)

Los Angeles, California 20

Chicago, Illinois $\quad 2 \quad 20$

Houston, Texas 20

Dallas, Texas 110

Atlanta, Georgia 110

Largo, Maryland 110

$\begin{array}{lll}\text { Philadelphia, Pennsylvania } & 1 & 10\end{array}$

Primary Target population (Client)

characteristics*

No specific target

220

MSM and some transgender mainly 40

MSM and some transgender mainly $\quad 2 \quad 20$

Heterosexual men mainly $\quad 1 \quad 10$

$\begin{array}{lll}\text { Some PWID } & 3 & 30\end{array}$

\section{Types of PrEP services provided}

$\begin{array}{lll}\text { Non-clinical } & 7 & 70\end{array}$

Clinical $3 \quad 30$

Notes: * Primary target population total does not equal $100 \%$ due to overlap, as some participants had more than one primary target 
Table 5 Becoming one with the community: A Dynamic Process of ASO PrEP Engagement and Outreach with African Americans.

\begin{tabular}{|c|c|c|c|}
\hline & \multicolumn{3}{|c|}{ Phases } \\
\hline & $\begin{array}{l}\text { I. Grappling with } \\
\text { pushback/challeng } \\
\text { es }\end{array}$ & $\begin{array}{l}\text { II. Transforming } \\
\text { challenges into } \\
\text { opportunities }\end{array}$ & $\begin{array}{l}\text { III. Establishing } \\
\text { authentic presence in } \\
\text { community. }\end{array}$ \\
\hline $\begin{array}{r}\text { Tasks } \\
\text { related to } \\
\text { respective } \\
\text { phase }\end{array}$ & $\begin{array}{l}\text { Acknowledging, } \\
\text { understanding types/ } \\
\text { origins of pushback/ } \\
\text { challenges }\end{array}$ & $\begin{array}{l}\text { Devising strategies to } \\
\text { deal with pushback/ } \\
\text { challenges }\end{array}$ & $\begin{array}{l}\text { Building } \\
\text { rapport/gaining trust } \\
\text { with community }\end{array}$ \\
\hline Transitions & $\begin{array}{l}\text { Pressure from } \\
\text { funders to meet } \\
\text { goals; motivation to } \\
\text { make an impact }\end{array}$ & Community buy-in & Diminished rapport/trust \\
\hline Conditions & \multicolumn{3}{|c|}{$\begin{array}{c}\text { Resources (funding, capacity e.g. personnel, educational and support } \\
\text { services, decision-making power) } \\
\text { Interactions (client/community) } \\
\text { Acronym: TRRI }\end{array}$} \\
\hline \multirow[t]{2}{*}{$\begin{array}{r}\text { Subcategori } \\
\text { es: Factors } \\
\text { impacting } \\
\text { engagement/ } \\
\text { outreach }\end{array}$} & $\begin{array}{l}\text { (i) Psychosocial } \\
\text { and behavioral } \\
\text { issues }\end{array}$ & $\begin{array}{l}\text { Addressing } \\
\text { psychosocial and } \\
\text { behavioral } \\
\text { factors }\end{array}$ & $\begin{array}{l}\text { (i) Understanding and } \\
\text { prioritizing } \\
\text { communities' needs } \\
\text { and struggles (e.g. } \\
\text { Matching personnel } \\
\text { to population } \\
\text { characteristics) }\end{array}$ \\
\hline & $\begin{array}{l}\text { (ii) Cultural/ } \\
\text { Societal factors } \\
\text { (e.g. cultural } \\
\text { norms/ societal } \\
\text { influences on } \\
\text { HIV/PrEP, } \\
\text { sexual norms } \\
\text { and values, } \\
\text { stigma, } \\
\text { historical } \\
\text { medical } \\
\text { mistrust) }\end{array}$ & $\begin{array}{l}\text { (ii) Addressing } \\
\text { cultural/ Societal } \\
\text { norms around } \\
\text { HIV/PrEP }\end{array}$ & $\begin{array}{l}\text { (ii) Being rooted in } \\
\text { community; ie. } \\
\text { Meeting people } \\
\text { where they are (e.g. } \\
\text { Avoiding } \\
\text { oversaturation/overl } \\
\text { oad of services) } \\
\\
\text { (iii) Maximizing } \\
\text { gatekeeper/ally } \\
\text { connections }\end{array}$ \\
\hline
\end{tabular}


(iii) Systemic and structural issues

(e.g.

Cost/insurance, staff buy-in) cost/insurance, integrating PrEP organizational philosophy to elicit staff buy-in

Understanding and defining a problem and its causes is recognized as a first step in the development of public health interventions which are defined as "planned actions to prevent or reduce a particular health problem, or the determinants of the problem, in a defined population” (Wight, Wimbush, Jepson, \& Doi, 2016, p. 520).

Figure 13 Becoming One with The Community: A Dynamic Process/Context Specific Framework for ASO Successful PrEP Implementation with African Americans.

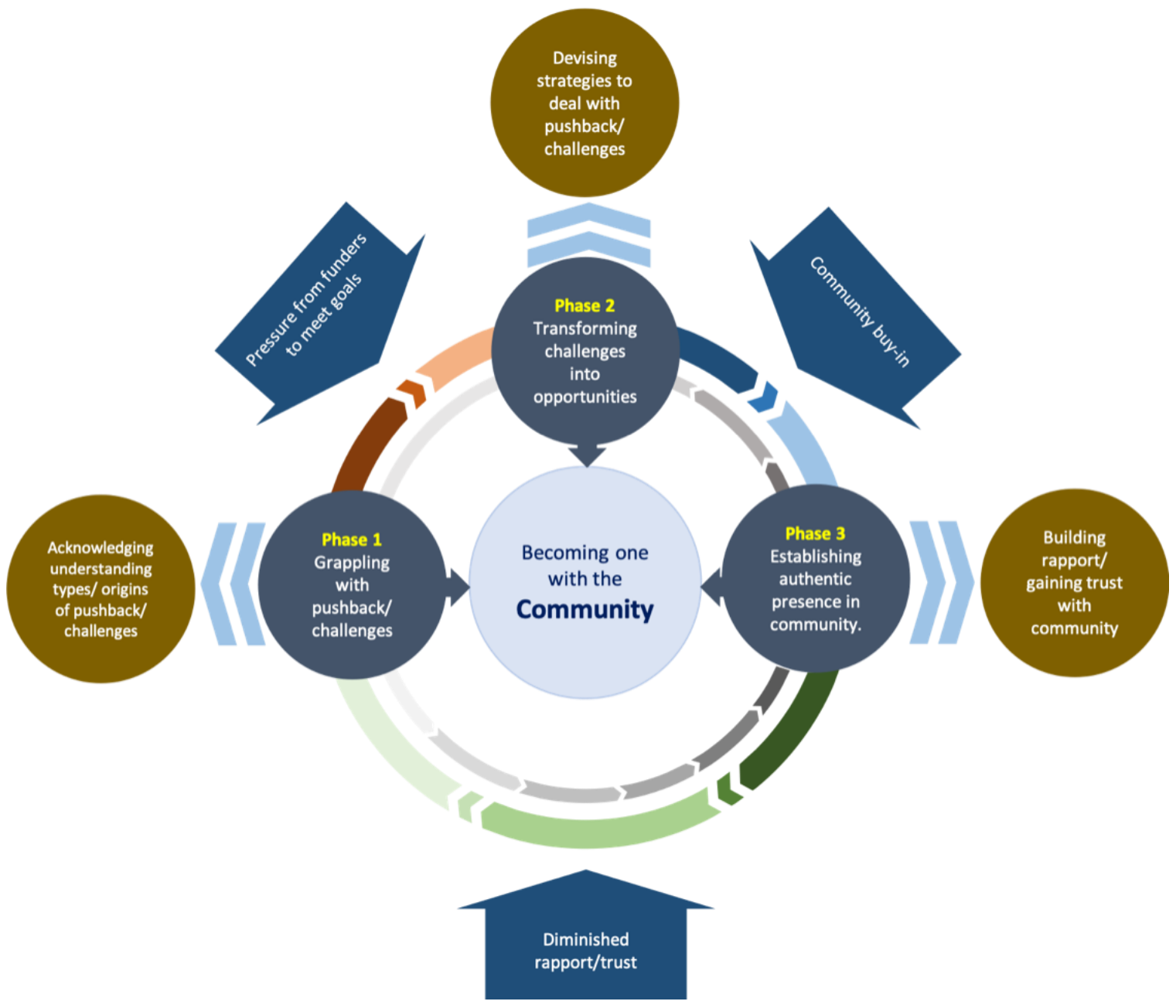


Consequently, participants articulated that pushback not only came from clients/target populations served, but also from within the organizations themselves, expressed as reservations about PrEP held by organization staff. Factors articulated by participants that related to pushback and challenges originating from within the organization as well as from the target populations served are grouped according to common occurring themes and presented in the framework as sub-categories: intrapersonal; sociocultural; systemic; and structural factors precluding African Americans from engaging with and using PrEP.

\section{Psychosocial and Behavioral Challenges to PrEP Outreach}

As part of phase I, participants consciously witnessed the interplay among personal and interpersonal factors like attitudes, perceptions, and beliefs among their clients and potential clients as well as how these factors influence the decisions of their African American clients with respect to PrEP engagement and outreach. Further, participants observed multiple barriers and reflected on the differences (gender and sexual orientation) among clients as opportunity areas of intervention. Some of the most salient ones are noted. For example, profound levels of low PrEP awareness, low knowledge, a lack of interest, and misinformation were prevalent due to many African Americans not adequately engaging with HIV prevention promotional materials within their community. One client stated,

"Unfortunately, most of them have little knowledge of it. And that's another one of our barriers, is outreach and getting the information out and actually getting them to read it. Because sometimes you can have pamphlets and billboards and 
whatever and they don't pay attention to it, it doesn't apply to them." P9_LosAngeles, CA

This pervasive lack of knowledge and understanding about PrEP, coupled with misinformation, was further observed to engender inappropriate uses of PrEP, especially among African American heterosexual men.

"And then when we get into populations where they are heterosexual man or a heterosexual men of color, and we find that it's used as like birth control and it's an option to condom usage and not being used together. So we have to combat that stigma [around PrEP] and decrease those misconceptions to make sure that those persons have the right information." - P4_Houston, TX

Participants also observed low perceived HIV risk and low perceived need for PrEP as major barriers. They noticed that differences in risk perception existed among the population (based on gender and sexual orientation). Heterosexual men, for instance, demonstrated lower HIV risk perception compared to women; women were less resistant and more willing to engage with prevention. Additionally, African American men who identify as heterosexual individuals, but who secretly have sex with men, reportedly shied away from using PrEP for fear of retribution from significant others who would question them, if the men were seen using PrEP.

"For down-low men [these are men who identify as heterosexual but who may have sex with other men], it'd be real hard because most of the men that I test that have girlfriends and wives, they definitely don't want to get on PrEP because they don't want their girlfriend or their wife to find out they're taking this medication because it makes them look like, "what do you need this for?" That 
makes them have to tell on themself. It's a little harder for the down-low population to be put on PrEP. It's real tricky. They're already in denial, so it's a little harder for them. Well with women, if they don't have a lot of sex partners, they pretty much feel like they don't need PrEP. If they know they do have a lot of sex partners or multiple partners or they think that their partner has a lot of partners. Once we let them know that there's a pill you can take every day that'll greatly reduced chance that you contract HIV, they really be more open to getting on PrEP if they feel they fit that need. A lot of black gay men do know about PrEP is just getting them to actually test.”- P6_Dallas, TX

Not only did participants observe differences in risk perceptions among populations, many of whom were in denial of their HIV risk, participants also noticed that many clients within the African American community endorsed conspiracy beliefs. Thus, these areas too were recognized as pertinent intervention focus areas to reeducate the community to correct the misinformation.

"A lot of that [LGBTQ] community has already heard of PrEP, you know what I'm saying? So it's not foreign to that group of individuals. And then there are some heterosexuals that have heard of PrEP, but there are a lot more that haven't because it doesn't, it doesn't pertain to them, so a lot of the communities in which we go and we work in and provide prevention, a lot of the communities, individuals are in denial or they're misinformed. You know what I'm saying? They're misinformed. Or they believe, we're still dispelling myths that, for example, two myths, I can just tell you two things I always hear. There is a cure for HIV because Magic Johnson got cured. That's not true. That's 
misinformation. Or the other one being only people that are very, very high risk people that are, what's the word I'm trying to say? I want to say it in its proper context. People that are being whore mongers, are at risk of HIV. Just because I'm with one person, I'm not a risk. but yet you're having sex unprotected with one person, you're having sex with one person, but how many people is that person having sex with?" —P10_Largo, MD

Recognizing these various intrapersonal and interpersonal challenges and their varying occurrence across client demographics helped participants to understand how to tailor their interventions to specific target/priority groups to address the HIV and PrEP education needs of their various clients.

\section{Cultural/ Societal Norms around HIV/PrEP}

Understanding cultural and societal-level influences on African Americans' PrEP engagement and outreach was critical for participants in this phase. Participants specifically emphasized the importance of being cognizant of the existence of stigma, historical medical mistrust, homophobia, and sexual taboos within the African American community. Acknowledging the historical unethical treatment of African American persons in research as well as continued experiences of disparities within society and differential treatment within the health care system was apparent in the data. Consequently, participants reported that medical mistrust was one of the greatest pushbacks to PrEP they faced from most of their African American clients. They observed that many of African American clients were unwilling to use PrEP for fear of side effects and long-term effects of the medication on their body, concerns which were born from a mistrust of the health care system. One participant remarked, 
"The biggest reaction is why? "Why do I need this and other people aren't taking this? Are you offering White people this too? Why do I have to take a pill? Isn't that going to kill me? Isn't that going to kill my lung or my kidneys? I read somewhere that it's going to affect my bones." It starts really deep; it starts with a lot of mistrust...And for populations that have a mistrust of the medical system, have a mistrust of chemicals, have a mistrust of, or an inexperience of utilizing medication on a daily basis, it's a transition for them to move from the state of readiness. The state of, "I'm thinking about it, this might be an option for me," to, "Oh, I can commit to this."”-P3_Chicago, IL

One participant further cautioned that it was important not to underestimate or belittle the fact that medical mistrust exists within the African American community, especially given the history of mistreatment of African American persons within the health care industry.

"To be quite honest, that medical mistrust is real. It's valid. People are not crazy because they think things because there's been medicine and medical doctors with a history of doing horrible things to us [African Americans] so it makes sense in some ways."-P8_Los Angeles, CA

Along with medical mistrust, stigma too was reported as being prevalent among African American clients and acted as a barrier to PrEP engagement. Various aspects of stigma were at play within their client communities. Stigma associated with being gay was demonstrated as clients' perceptions that they would be judged or shamed by their referents (family, friends, significant others, etc.) for being gay. HIV-related stigma was observed as persons within the community having judgmental attitudes and negative 
opinions regarding HIV and persons who have HIV. PrEP-related stigma was implied by clients' fears of being judged or shamed for taking a pill (PrEP) associated with HIV. "I think fear. There's a lot of fear in a lot of ways. Fear about HIV, fear about PrEP, fear about having to go to the doctor regularly. I have people who don't like the idea of they have to go get checkups every two or three months. Just fear about stigma. People are fearful about where they're going to have the medicine delivered. A lot of people live at home with their parents or with roommates who don't even know that they are gay, let alone taking pills to prevent HIV, so a lot of fear of the stigma. It's really what drives a lot of people.”-P8_Los Angeles, CA Some participants located in the southern U.S. felt that stigma was especially apparent in the south. They further asserted that being in the South required them to constantly battle with stigma around HIV. The participant seemed to allude to the conservative nature of southern U.S. states where communities may frown upon open discussions about sex and endorse homophobic attitudes and misperceptions that PrEP is only for men who have sex with men. One participant indicated,

"We definitely still feel a lot of stigma. We are in the South and the South is just unfortunately disproportionate. We affected compared to the rest of the U.S. So stigma is definitely one of the things that we have to battle and we continuously battle and fight against. So some of the feedback that we received, it's just, as I mentioned pushback, you know, people think that it's really not for me because I'm not part of this pocket of, of, of a label. And that's one of the reasons why we don't target specific groups. Because if you do, then you are consciously or unconsciously putting them into labels and, and that kind of sometimes 
contributes to that stigma because, Oh, if you're offering me PrEP, but I hear that it's supposed to be for MSM, you are either assuming that I am an MSM or, or you're targeting me because of who I am. So, we really focus on eliminating labels and in order to do so we've really just have a blanket approach that this is a service that we're providing for everybody. — P5_Houston, TX

Overall, most of the pushback participants experienced appeared to have deep roots in cultural and societal norms upheld by the African American community as well as discourses within the society. For instance, poor interaction with sexual health interventions and poor HIV risk perception are born out of community-held beliefs around HIV and sex. Within the African American community, talking about sex is considered a taboo and so it is not openly discussed within many households. There is also a lot of stigma around HIV, and that negatively impacts the community's willingness to engage with HIV-related promotion for fear of being stigmatized. Thus, many do not want to be seen associated with HIV or medications for preventing HIV such as PrEP.

\section{Systemic and Structural Issues around PrEP Engagement and Outreach}

Systemic barriers were also ascertained to impact PrEP implementation. These included organizational policies and/or structure including staff buy-in, provider attitudes (including display of racism and discriminatory practices among health care personnel), cost and affordability of PrEP, insurance, incentives, transportation issues, complicated application processes for medication assistance program (MAP), and disparities in availability and quality of PrEP promotional resources within African American communities. 
Cost was articulated in terms of limited affordability of PrEP due to lack of insurance and low socioeconomic status of many of the participants' African American clients. This was especially seen as a concern since most participants dealt with transient (homeless) populations, many of whom not only lacked insurance but also did not see insurance as a priority. For many of these clients, survival was more important.

"When we engage individuals out in the field, most of the time they don't have a phone number, or they don't have access to a phone number, or their phone number is off right now, or that government phone, but they only get 15 minutes a month, so don't call them. Or they don't have insurance. They don't know how to navigate their insurance, they don't know how to get insurance, they don't know the importance of insurance. And for the hard to reach, it's more so that the populations are so focused on survival and the needs of survival that when you engage with them with anything that falls out of their hierarchical need of survival, it doesn't fall on their priority list." - P3_Chicago, IL

Participants indicated using medication assistance programs (MAP), as these are intended to help clients who lack insurance to deal with issues surrounding cost and affordability of PrEP. However, MAP were seen as a double-edged sword. On the one hand, they were a welcome solution to overcoming cost and insurance barriers. Yet, on the other hand, the arduous process of applying for MAP — the time-consuming nature of the paperwork - was enough to deter some clients from following through with obtaining PrEP.

At the organizational level, participants acknowledged that provider attitudes, in terms of staff-buy in, were important for effectively implementing PrEP. Some ASOs 
experienced initial pushback from staff due to staff having difficulty with making a shift from a focus on behavioral interventions (services that most ASOs were used to providing) to a more biomedical model of emphasizing PrEP. Some of these ASO staff did not believe in a biomedical intervention per se — "taking a pill to fix everything" as one participant put it.

"I think all of the team members that work in our preventative services, are prevention-based period. So I think even though from a personal standpoint, some of the individuals don't particularly believe in taking a pill to fix everything, they believe in changing the behaviors. They still are prevention specialists...?" P2_Atlanta, GA

These biases expressed by individuals within the ASOs were described as a challenge to implementing PrEP that ASOs recognized needed to be addressed before they could effectively move forward with PrEP implementation. In attempting to ready their agency personnel for the transition to a biomedical prevention model, some ASOs experienced pushback in getting their staff on board with the idea.

"I think the bigger issue was getting staff on board and understanding all the things of PrEP, and then making sure we make the referral and being able to answer the questions of the individuals who are at greatest risk and who are interested in PrEP. I mean, you know.” — P2_Atlanta, GA

Consequently, even though they understood the efficacy and importance of PrEP as a step towards achieving zero transmissions, some participants still interpreted the idea of using PrEP to prevent HIV "force-feeding a pill" on people, and thus expressed reservations about the idea. 
"I feel like the difficulty with biomedical intervention as the only option, is that it is somewhat force-feeding a pill on a population, and it's taking out the fact that there is a lot more work that goes into just taking a pill every day. And I feel like a lot of people who have worked in the field for so long, they see PrEP as such a wonderful solution. And it is such a wonderful, it is a good tool to get to a potentially a functional care or to Get to Zero. But if you take out that behavioral aspect of it, you're really missing the primary focus of why that client is there." P3_Chicago, IL

It is important to note that many of these ASO representatives working with these clients are themselves African American, so the ASO representatives, too, may have shared experiences with the larger African American community (of being leery of the health care system) and thus needed to first grapple with their own personal biases and examine them in context of providing care for their community. For some, that shift in mentality needed to happen first before they were ready to begin advocating PrEP as an option for HIV prevention.

\section{Phase II. Transforming Challenges into Opportunities}

The transition to phase II happened when participants recognized that they needed to overcome pushback and challenges that preclude engagement of target populations if they were to make an impact in the community as well as meet funding goals. Given that most ASOs are grant funded, they are often under pressure to meet grant goals. Thus, it is no surprise that participants would need to devise ways to overcome those challenges to improve their output, hence their transition into the next phase of the framework. 
Phase II is characterized by strategies that participants employed to help them get navigate barriers to PrEP outreach/service delivery encountered during phase one. These include strategies for addressing psychosocial factors; navigating systemic and structural factors (e.g. helping clients navigate insurance issues, MAP applications, addressing other competing priorities, offering clients a "buffet of options," embedding PrEP within other services, providing agency-wide training to elicit buy-in); and cultural beliefs (e.g. addressing stigma and medical mistrust through various means like educating/re-educating the client population).

\section{Addressing Psychosocial and Behavioral Pushback/Challenges}

Participants utilize several strategies to navigate and address the pushback and turn them into teachable moments. Education and "reeducating" the community was one of the ways participants dealt with the low perceived need for PrEP and misinformation about PrEP. Participants believed that education is the foundation for correcting many of the intrapersonal level factors that contribute to pushback observed among clients such as the low knowledge, awareness as well as the pervasive misinformation and low risk perception within the African American communities. One participant stated,

"We start with the foundation of education. We believe education is the start of everything, so we provide health education and risk reduction every time we're in a field, whether it's an event, whether we're out utilizing our mobile home offering free testing. So we provide the health education of risk reduction practice to individuals, then educate them, number one about HIV, and then to show them 
how no matter what type of sex that they may be engaging in, how they can do it as safe as possible." - P10_Largo, Maryland

Persistent engagement of clients in conversations and multiple education sessions was noted as an effective strategy for improving clients' risk perception and willingness to use PrEP among clients who were, hitherto, were either unknowledgeable or skeptical about side effects. The practice of persistently educating clients helped clients recognize their risk of HIV and transition to accepting PrEP as an option for HIV prevention.

"For the individuals who've never heard about it before, they are really skeptical because they haven't had the information up close and personal or some individuals who have been on the fence about whether or not PrEP is going to work for them. After a few conversations, you could see them turn around and says, okay, here are my risk factors and as a result of my risk factors, that maybe I do or maybe I should give it a try. So, the willingness I'm finding that the willingness is increased after multiple sessions involving education. "P10_Largo, Maryland

Participants further determined that consistently educating individuals and community at large was necessary for normalizing HIV and engendering change both in the individuals as well as within the community.

"Unless people are exposed with the information, you're not going to have some type of change within the individual or even the community that they live in. So making sure that we continue to, to provide this information." — P5_Houston, TX 
"I think what we do is, we try to reduce some of that community fear, by normalizing and talking about in a way that gives people hope." -

P7_Philadelphia, Pennsylvania

\section{Navigating Cultural/Societal Norms around HIV/PrEP}

One of the ways that ASOs were responding to these issues relating to factors like racism, segregation, discrimination, and stigma was by acknowledging that these exist and really do impact participants and calling for the systems to address these. Also, to combat stigma, participants are engaging in activities that help to normalize HIV and PrEP among sexual minorities within the community. For instance, they are being conscious of how they present information by paying attention to language (e.g. what are they calling their outreach? What taglines are they using?)

Since HIV is highly stigmatized, participants believed that a strategy to destigmatize HIV would be to normalize it. Thus, some ASOs resorted to removing HIV from all sexual health promotion. They recognized that HIV is not acceptable within segments of the African American community thus, including HIV in promotional materials would only perpetuate those stigmas. Instead, the ASOs focused on providing "whole health", that is, promoting sexual health services along with other screenings while integrating HIV services within these services.

"And so, what whole health is, essentially, is that we are providing not only sexual reproductive health care, we're also providing care related to blood pressure, right? So we'll do blood pressure screenings, we'll do STI screenings, as well. Because we know that if we promote or publicize HIV testing, there's going to be quite a few people that are turned off on it. And so, we tend to remove HIV 
from our tag lines and our promotion, because we don't want to stigmatize an already stigmatizing situation._-P7_Philadelphia, Pennsylvania

Additionally, participants recognized from their interactions with the community during phase I that some of the cultural barriers to PrEP engagement observed among African American communities were borne from a lack of adequate information about HIV as well as sexual health. Many of their African American clients had severely low levels of PrEP awareness and knowledge, and high levels of misinformation, which may have contributed to those clients' endorsement of conspiracy beliefs and stigmatizing views about HIV and PrEP. Thus, participants decided that consistently educating the community would be a good strategy for changing cultural and societal norms.

"But on a community education level, it's just a matter of making sure the information is out there. You're not going to achieve a change in societal norms or even community norms unless they are exposed to the information whether or not they like it. And so we are okay with getting pushbacks and we'll continue to promote whatever we need to promote this to make sure that they understand that this is simply another option that's available.”- P5_Houston, TX

\section{Navigating Systemic and Structural Issues}

Recognizing that many of their African American clients were profoundly disenfranchised and lacked the wherewithal to afford PrEP, participants resorted to finding ways to help clients access PrEP more easily. Participants utilized medication assistance programs (MAP) to help the uninsured overcome cost and affordability barriers. For instance, most of their clients lacked access to important necessities like transportation and health insurance, important indicators of socioeconomic disadvantage. 
Some participants even went out of their way to expedite MAP application processes to improve client experiences and to reduce any unnecessary added burden.

"And there are a lot of barriers, transportation, medical knowledge, whether they have insurance or not. Most of the patients that we see are uninsured and so we have to use patient assistance programs to get their medication covered. So it can be very difficult. But we have case managers and I do a lot of my own patient assistance program application works so that I can help expedite that, so that the patient isn't waiting on us. And then once the application is approved and they get the numbers from them and then they take it to the pharmacy and then they provide the pharmacy with the numbers that they get so that they can then have their medication covered. So that in itself is a barrier because all those extra steps and all that type of stuff is something that would prevent somebody from taking that on."-P6_Dallas, TX

Additionally, within the context of systemic barriers to PrEP engagement, ensuring that all agency staff understood the importance of PrEP was recognized as being incredibly important for effective PrEP implementation. Agency-wide staff buy-in was encouraged to ensure that no opportunities were missed due to staff not all understanding the ASO's mission for PrEP. To accomplish this, participants were highlighting organization's PrEP goals as part of the hiring process.

"So that's one of the things that we do with our hiring process and making sure that you understand, Hey we're, we're promoting something that is not widely viewed as something that should be talked about openly or whatever may be. Number two is really making sure that you have a buy-in from the agency before 
you even start any type of education and promotion. Because if you don't have the buy in from the top down from the bottom up, whatever it may be, everybody needs to be on the same page when it comes to PrEP. I expect our organization for, you know, for clients who will come in and they go to the, to the x-ray tech and for them to say, 'Hey I heard about this blue pill.'" — P5_Houston, TX This step was important because participants recognized that staff had differing views about PrEP and endorsed personal biases that needed to be addressed to effectively provide PrEP services. For instance, some staff were keen on behavioral prevention options and did not personally endorse a biomedical option like PrEP. To complicate matters, some staff were influenced by the legitimation around PrEP and expressed safety concerns. Thus, it was imperative to adequately educate and train the staff on PrEP to decrease biases and improve client experiences.

"I think the bigger concern with PrEP is, there are many different train of thoughts as relates to staff. First and foremost, some staff members believe in PrEP and think it's great and some staff members are a bit concerned about PrEP because we're so old ethically, we still believe in people changing their risk factors, changing their behaviors. So, and then we've heard so much negative information about the medication and the class action suits against the pharmaceutical companies as relates to the liver damage and so on and so forth. So first having all the team members be on board with, we may not personally be a fan of it, but it's something that we need to make sure we offer to everybody and be educated enough to provide information to individuals." - P2_Atlanta, GA 
Thus, ASOs resorted to ensuring that all staff were properly trained and understood the organization's mission and goals concerning PrEP. They emphasized the importance of ensuring that all personnel within the agency, especially those directly providing PrEP services were adequately trained to do so effectively without being influenced by their personal bias.

"Make sure that, first of all, that you or the agency have a conversation with the people that are going to be making the referrals, about any biases that they may have. Have that conversation because the same biases that the staff members have, some of the people that they engage are going to have those and you want to be able to respond to that in a proper, properly. I mean, when we engage with any client and we do the whole prevention spiel, we talk about the risk factors. I mean, and that's everybody that's part of our prevention, our outreach team. And that's from our peer specialists, all the way to our managers, supervisors. There's just this whole spiel about, do you know how HIV is transmitted? You find a way to open up the conversation and it kind of takes off from there.” — P2_Atlanta, GA Further, participants recognized that organizational culture had a considerable impact on client experiences. They emphasized the importance of integrating PrEP into organizational philosophy and ensuring that organization staff, especially outreach personnel were trained and on board with PrEP. One participant noted:

"What is the pretty much creating an internal policy and procedure to make sure that you are able to draw out everything. Now we don't stop with just the providers because the providers are the ones that are able to provide the prescription, but it goes all the way down to even the front desk staff. If 
somebody shows up and says, Hey, I'm interested in PrEP, but if they don't know what that means, or they maybe they send a whole entire word, 'I'm interested in pre-exposure, you know'" — P5_Houston, TX

Participants demonstrated that it was not only it important to train outreach personnel in PrEP. It was also important to ensure that everyone else in the organization has some basic information about PrEP to improve client experiences.

\section{Phase III. Establishing Authentic Presence in Community}

The transition to this third phase happened when participants realized that if they were to make considerable impact and have sustained success in their PrEP service delivery efforts, they needed community buy-in. Accomplishing this required building rapport and gaining trust within the communities in which they served. Participants employed various ways of engendering support from the community that are presented under three subcategories: (a) understanding and prioritizing communities' needs and struggles (b) being rooted in community, and (c) maximizing gate keeper/ally connections. The more participants engaged in the tasks associated with this phase, the more they experienced success with the engagement and outreach efforts to their respective target communities.

\section{Understanding and Prioritizing Community Needs and Struggles}

Participants demonstrated that building rapport and trust within the African American community required prioritizing community needs and struggles. Participants attempted to accomplish this using the following strategies: making clients feel comfortable enough to be open to having a conversation and knowing the clients' needs (e.g. What types of needs are a priority to the client? What types of incentives are 
appropriate for the target population? Do food cards or clothing cards work better for them than a gift card? How tangible and appropriate are the incentives?). Participants advised that prioritizing needs of the community showed the community that the ASOs were not simply there to exploit them for grant numbers, but that the ASOs truly care. This is important because many priority populations have several competing needs that are higher on their priority list. Many of them simply need to survive. They need food, shelter, help with insurance, etc. Thus, when they are approached by ASOs, it is imperative that those needs are met before the client can be open to sexual health services offered by the ASO. One participant narrated their experience with this,

"Clients primarily come seeking services for one of the other reasons, like they want to get ... they need a food card, they need help with their insurance, they need help paying the rent or something like that. And then we engage them with preventative services in addition to the services that they're already getting...And so our primary mode of engagement is the other barriers to service that are a higher priority on a client's list. We work with a very hard-to-reach population. Primarily black and Hispanic MSM and trans black and Hispanic individuals. And when engaging with these populations, if you start the conversation with HIV test, they lose interest. I've also received lots of comments from people of community that feel like prevention services and prevention initiative have singled out populations, and youth populations, for funding and for numbers, and not to assist or affect change within that population. And so we try to address all those other barriers that are higher on the priority list to somebody, and then get 
engaged with them in sexual health and prevention services, and addendum to whatever services they're looking for.” — P3_Chicago, IL

Thus, this approach of first understanding and prioritizing client needs over those of the ASO contributed to rapport building for the ASOs and gaining of the community's trust. It ensured that the ASOs were viewed as genuinely caring rather than predatory simply seeking to take advantage of the populations to meet grant goals.

Participants also noted that in addition to meeting needs such as food, shelter, and daily necessities, it was equally important to be sensitive to other community needs such as preferences for engagement. For instance, MSM may not want to be approached in the open, so talking with your clients and letting them articulate their preferences for engagement helps to improve ASO client relationships. One participant remarked, “There's a lot that doesn't work well. If I can say anything, it's just to know your client. For example, when I came here I didn't understand the concept of, "Maybe someone doesn't want to be outed at school," like I said. I was going to school thinking I was going to get this huge outpouring. I was going to go to the LGBT groups on campus and meet all these cool people and it wasn't like that because A, people don't want to be outed as gay and then B, they see me walking around with a red HIV shirt on and they don't want to be associated with it. So that was an instance of me not knowing the client very well and maybe not planning as well as I could have for an event like that. Talk to your clients. Let your clients be heard, definitely first and foremost. Like I said earlier, just knowing your clients, knowing them. There's not a one-size-fits-all. What we do here is not what they 
do at [place] in Beverly Hills. Two completely different things. It's very important to just know your clients and plan accordingly.”-P8_Los Angeles, CA

Another participant also demonstrated meeting clients where they are metaphorically by being sensitive to the clients' background and experiences that may be related to the clients' gender or sexual orientation and may influence that clients thought process when with respect to PrEP.

"Well, we start by meeting the client where they are. So if there's a person who is a heterosexual African American male, we are going to meet him from that standpoint. So there's going to be certain things, certain experiences that he has went through that's going to formulate his thinking. So, we start there and then on vice versa, if we're talking to a heterosexual African American woman, her stigma, her misconceptions, her thoughts and experiences, may be totally different. So we build upon where that person is." —-P6_Houston, TX Another also highlighted using a variety of outreach strategies:

"We have different programs, like we use social networks, we use incentive in the community, we give gift cards for testing. We do, we give out information.” P4_Houston, TX

Participants demonstrated the importance of "meeting people where they are" such as using non-traditional efforts like the mobile van to reach clients who would otherwise not have access to services and using a rapid PrEP model to decrease barriers to obtaining PrEP. Both approaches were shown to increase success with PrEP uptake. One participant reported, 
"Yes. So, our outreach, our engagements have increased with inclusion of mobile, of our mobility, and our tele PrEP. Tele PrEP launched a month ago, and within a month we've linked 15 people to PrEP. The month before we averaged around five a month. So once we got that rapid system going on, it almost tripled. And so we're hoping to continue to see that. That's everything, that's actually people coming in having received or known some information about us. So that outreach efforts, yeah, from an outreach efforts, it's nothing but improved by having appropriate incentives, by having the rapid model, and being able to meet them in their community." — P3_Chicago, IL

Further, understanding that African Americans are more likely to trust others who look like them, participants ensured the use of outreach personnel teams whose characteristics matched those of the target demographics whom they served. This was seen to be more effective than not having people with whom many African American clients could identify. To accomplish this goal of matching outreach personnel to community characterizes, participants hired outreach personnel from the African American community. This not only ensured that ASOs could connect better with community, but it also helped them build rapport and engender trust among the community.

"We also employ people in the community, too, that look like the population we're serving. And I think, because of that, it helps us to build more rapport and trust in the community, I guess, so that this comfort or anxiety around research is not so prevalent." — P7 _Philadelphia, PA 
Participants, however, cautioned against simply hiring from community, as this could backfire - be misconstrued by community as predatory on the part of the ASO. They stated that hiring from community may seem like a benign strategy from the standpoint of the ASO. However, the community may not look favorably on this, if they perceive that the only reason people from the African American community are being hired is to help the ASO meet their grant goals.

"Government funds like [federal and local government grants] or any of them, when they fund cooperate, or they fund these agencies, they target a population, they say you need to reach 2000 black MSM individuals, right...Now, to reach 2000 black MSM, what do the community-based organization have to do? They have to hire people of community to reach out to people of community. So, you've got organizations that are hiring black MSM to reach out to black MSM. Now, those black MSM that are hired by these organizations, because of this grant, is the only reason why they're being considered for the position. They're only being considered for this position because they're Black and MSM.

Secondly, they're being considered for an entry level outreach or position. So, not only are we taking community members and employing them, but we're only giving them entry level skills and abilities. So, because they have only entry level skills and abilities, these organizations that are claiming to hire from community and raise community, essentially only helping support the system that's oppressing them, right? So, the effectiveness shifts from, I want to make a difference, I want to reach these people to, oh shit, I need to reach 2000 people before the end of the year. And when that mentality shifts, the quality of those 
engagements diminishes. And this is historical. Throughout time, people in these communities have come to realize that these organizations are only using them to complete their numbers so they can maintain grants.”- P3_Chicago, IL

Participants implied that it was not enough to simply hire from community; the ASOs needed to invest in the community past the entry-level training that ASOs typically provide for the community members. ASOs need to show the community that they truly care. Participants alluded to the importance of ASOs hiring community members to occupy positions beyond entry level as this practice would show the community that the ASO is truly invested in the wellbeing of the community and not simply in meeting organization goals.

It should be noted that when participants were unable to match outreach personnel characteristics with population, they employed other strategies to compensate this limitation. For instance, participants indicated it was important to have a diverse team of outreach personnel to pull from even if the specific demographic characteristics were not possible to match. Specifically, they suggested having a team member who possesses other qualities with which the target population can relate.

"If we can't find those specific characteristics, we go to somebody who is able to exhibit something that the client hopefully is able to click and engage with. So, depending on how big a team is, having an array and a variety, of staff members that is diverse, can definitely help. Because when I get request, uh, for us to do community events, I have to be very mindful of who I send. And I'm grateful that I have such a diverse team, that's almost a buffet, right? Like there's like a buffet 
of options that I can pick from. It's going to be you or it's going to be you so, yeah.”_- P5_RT_Dallas, TX

\section{Being Rooted in Community}

For participants, being rooted in the community meant making their presence known in the community. This required them to engage in activities that helped to establish the ASO's presence within the community such as not waiting for clients to come to them, but literally "meeting people where they are," hitting the streets or taking outreach to the streets, going into physical spaces, like clubs, bars, barbershops and being consistent in their approach using non-traditional reach out methods like social media and mobile vans, driving around town. Participants emphasized that meeting people where they are, that ,is in spaces where they feel comfortable, demonstrates care on the part of the ASO and thus contributes to building rapport with the community. One participant stated,

"I think the organizational structure here is really meeting people where they are, and then, trying to give them the resources that they need, to make sure that they get linked to services that they need, right. So, one of the things we do is, we do give gift cards. We do go out in the community to drug and sex trade areas, and we have, on our mobile unit, packs of cookies, or juices, or whatever. Because we're trying to, one, make feel people good, and not being in their area, look down on them. But come, giving them, what they, essentially, they need. And oftentimes, people that come to us to get tested, haven't had a meal in a few days. So, we want to make sure that we're removing some of those barriers. And in the 
hopes of removing those barriers, and really providing comfort, we're building that rapport.” — P7_Philadelphia, PA

"Showing up in spaces where young people are," such as meeting people in virtual spaces like social media or through web-based methods, also contributed to rapport building and success with outreach. One participant described having significant success with outreach using Snapchat and Instagram by maintaining a strong daily presence on the web. When asked, "How would you say your outreach efforts have impacted your progress with PrEP?", the participant responded,

"Since we have been able to go do more web-based social media outreach, like I said, we've been getting much more people that have come to the Empowerment groups. The first Empowerment event we held was in September and I think there was maybe, I don't know, 12 to 15 people there. And at the end of that month is when we started to do the web-based stuff. We went really hard at it. And our most recent one on Thanksgiving, like I said, we had our Linkage to Care coordinator there connecting people as they were walking out and there was almost 30 people. So web-based has really, really helped. Having a really powerful web presence on Snapchat, Instagram, actually posting on the stories every day. What I try to do is I try to post what we're doing in the office to prepare for events. So, yeah, web-based outreach is huge. Web-based outreach has definitely contributed." - P8_Los Angeles, CA

Being rooted in community also meant being present and showing up consistently to multiple events within the community. This consistency ensured that ASO's presence was registered in the community and, as a result, community members became more 
willing to engage with ASOs during subsequent visits. This practice of repeatedly showing up was shown to build trust and break down barriers of stigma that have been etched on the minds of many African American communities. Thus, participants succeeded in reaching many who previously would not have payed attention to the ASOs. One participant stated,

"That [consistency] looks like if there are three community activities, if we're at two of those three, then that create a stronger visual presence for us in the community. And what happens is the individual that live in that community sees that presence and they says, okay, well let me engage with this organization. Because every time I look around, they're there. So it provides trust and that breaks down a whole lot of the stigma. And then after the breakdown happens, then we're able to get in and penetrate and really, really engage individuals within the community." — P10_Largo, MD

Another participant also demonstrated success with community event-based outreach. Through this approach, many community members came to use PrEP.

“Our outreach efforts, they have been very successful. They've brought us in a lot of participants that do end up getting on PrEP. But like I said, it's usually event based. If it's just a general outreach, it doesn't seem to have as big of an impact. But definitely when our outreach is wrapped around an event, we have great success, usually." -P2_Atlanta, GA

Another also emphasized the importance of embedding PrEP outreach within community-based events, especially when dealing with youth. 
"I guess the least of those would be maybe just doing ... I don't want to call it like basic outreach, where we just kind of go out and if there's not an event wrapped around it, it tends to be less successful. Like especially since we deal with the youth, youth are all about music and food and excitement and things that. So, we try to keep that kind of energy when we do approach them. And a lot of times, that means that's putting on an event or something, but when we don't put on an event, we just kind of do general outreach, I don't think we get as good of a response.”-P9_Los Angeles

Furthermore, community mobilization around PrEP through events hosted within the community by ASOs were shown to be highly successful in drawing the attention of the community to HIV prevention and disabusing the minds against PrEP of the community "I do think there is needed mobilization in the community. And so, here at [interviewed organization], for instance, last year, we hosted the first ever PrEP Week. PrEP Week was for us to really celebrate the birthday of PrEP. And so, on July 15th, or the 16th, the FDA approved Truvada for PrEP. And what we did was, we made it a week-long celebration, and we brought together people from all over the Tri-State area, we brought a news media. We had a great host. We did several different events, to create awareness around PrEP. And as a result of that, we were able to have 40 new PrEP starts that week, and re-engage 20, those who are on PrEP, or lost to care.” — P7_Philadelphia, PA

Not only did participants demonstrate success with being "active in the community" by hosting and attending multiple events within the community, but they also demonstrated oneness with the community by having an open-door policy where community members 
were allowed into the ASO's space anytime to receive help with various needs including non-health related needs like resume' and job application assistance.

"Well, because we are active in the community and the people do see us, they see our logo, they see us. Like back to school, we give out backpacks, school supplies. We're there in the community, so when they see us, when they see a mob, I mean people get really excited because they know that we're bringing something good to the community and they look forward to seeing us, actually. And just the events and just being visible. And we have a drop in Wednesday, where they can come in and use our computers and we help them with their resumes and anything else that we can help them with. And so I think just having our doors open, being welcoming and being visible, we have found much success in that approach... But I think to be better effective is to be on the same level, is to be in their neighborhood. And you have an office in their neighborhood and they can come into your office at any time and get services. And get other incentives, if they need bus tokens, anything like that. So, I think just being one with the community and being visible.” — P9_AILosAngeles

Part of establishing authentic presence within the community was described as making conscious effort to avoid oversaturation of services within a community by fostering partnerships and working in collaboration with other organizations.

"The number one for me would be to recognize who your community partners are and see who is doing similar work. Because one, you don't want to duplicate any of the work that you're doing. Two, you don't want to oversaturate because once you start doing a hard promotion of whatever it may be, people get turned 
off. So, making sure that you have a clear understanding of who is doing what. And, and for maybe for some cities, and luckily Houston is not, not so much this, at least not at [interviewed organization]. You know, not everything is a competition, you know. So, if you have another AIDS service organization providing the same thing, great. That just means that there's more people all with the same goal. So, ensuring that you know, who is fighting alongside with you, look at their strengths, look at their weakness, and look at your own strength and weakness and just make sure that you're not duplicating any efforts. And, and there's a sense of agreement of what is the collaborative approach that we are going to take. As it relates to PrEP."- P5_Houston, TX "Some other organizations, some people that may be, for instance, we have this one, it's a shelter and they work with young adults, so we go out and we'd speak with them, just so they can have, they may not offer the services that we offer, so they have us come in and speak to those services and provide education on those things. They may just be providing housing, you know what I'm saying? But they want the individuals to get more knowledge and information on a variety of other topics."-P2_Atlanta, GA

Participants implied that overly saturating a community with HIV or PrEP promotion may become overwhelming for the targeted population.

"I think, also, dealing with black gay men, or MSM, in the community. It's difficult in a sense, too, because I think the HIV testing services and PrEP, our promotion, has essentially been thrown down their throats. I'm noticing this with MSM communities.”-P7_Philadelphia, PA 
Participants advised that having a mutual understanding among organizations regarding expertise would decrease the likelihood of duplication of services that would only serve to overwhelm the community.

\section{Maximizing gate keepers/Ally connections}

Gate keepers and allies were considered entry points or liaisons into the community. Taking advantage of this resource to improve community engagement requires the ASO to identify the gate keepers/allies, establish trust with them, and then rely on gatekeepers/allies for connection and insight into the community. Participants identified gate keepers as peer group leaders, community leaders, existing clients or nonclients who are community members, and community advisory board (CAB). Other community organizations with whom ASOs collaborated for PrEP engagement were considered as allies. Selection methods varied per gatekeeper type. Some organizations had existing programs from which they could draw community members to serve as gate keepers. Other ASOs solicited gate keepers within the community by advertising and asking for volunteers to serve as gate keepers for their community and to receive an incentive for their participation. CABs, for instance, comprise community members mostly from the priority populations selected and paid an incentive to serve for a period of time to advise the ASO on matters relating to engagement with the community. One participant discussed using a $\mathrm{CAB}$ and how they select their $\mathrm{CAB}$ :

"Every time we have a new $\mathrm{CAB}$ [community advisory board] cohort, basically we reach out to multiple of our programs. So, our CAB can be composed of clients and non-clients. So, like I mentioned before, we have chapter community advisory board, that every, that has a new cohort, six, four times a year and it has 
a total of 10 to 15 people. So through chap [interviewed organization's apprenticeship program], who are all from community, all primary populations, we're able to reach out to them and provide them with compensation for their input, right. So, one third to one half of our $\mathrm{CAB}$ is composed of clients from other programs that fit our demographic. The other half, we do post externally through geosocial, through social media. And we do ask that, we reach out and say any individuals of color, of the queer expression who are interested in providing feedback and support to [interviewed organization] can receive compensation... for their participation" — P3-RT_Chicago Another participant mentioned a different type of gatekeeper strategy, the "social network strategy." This type of a peer-oriented strategy was also instrumental in connecting ASOs with priority populations through referring of their friends.

"The allies are other individuals in the community. We rely on what we call SNS, which is social network strategy. And what that does is it allows us to have intimate conversations with small groups that will allow us to create a more intimate setting and people are more open in intimate settings. The allies identified a lot using basically their interests. They really come to us, like say for instance, if we come across like a new positive during that conversation, that rapport that's being established, it often comes out if they have a cluster of peers who may be at high risk, who wouldn't mind engaging in such conversations." P10_Largo, MD 
Similarly, another participant reported using a peer referral strategy, although less formal than the SNS. They simply identify peer group leaders from within the various priority groups in the community who then bring their friends with them.

"They bring in their friends, they have friends that we don't ever see in a club we don't see in the community too much. So we have very popular people who as well, influence over a small group. We get them to get with their friends and their friends' friends and it worked, because those are people that we probably wouldn't have, we would've missed. Right. I think that's really, definitely important because of, you know, they'll listening to them versus us a lot of times." - P6RT_Dallas, TX

A third recommended strategy was utilizing the Internet to locate popular opinion leaders (e.g. DJs, promoters, popular club and bar hosts) within the community.

"I mean just being out on the scene, knowing who is popping on internet and the one who was popular in the club, DJs, um, promoters, um, people who do, who host the club events, who on the mike, bartender, uh, you know, just people is very popular that we noted. You know, a lot of people will look up to, well, you know, just very positively." - P6-RT_Dallas, TX

Fourthly, participants recommended identifying allies by scouting the community and seeking out opportunities to build collaborative partnerships with existing entities and organizations within the community. Special emphasis was placed on entities invested in a common goal of improving the community, such as churches and other places of worship. One participant said, 
"You, identify community partners or entities or organizations or churches, you know, whatever it may be, and just go one by one and say, 'Who can I speak with? This is what we offer. Can I provide you with just some information, or, if not, you know, can we establish a relationship' That's what's worked for us to the point where we even have established MOU where this is where this is like the legit agreement between you and I, but we want to make sure that we can do as much as we can for your community folks. So less with the surprising. I don't want to shake hands. I don't want to simply say 'We're going to do this.' Let's make the legit, you know what I mean? It's really that grassroots approach. Literally looking on Google or doing a drive by and finding out, 'Oh, that might be a good place! Let me find out what they do! Oh, I know this other place. Let me find out what they do.' Yeah. So really, nontraditional grassroots approach to making these immediate connections." — P5-RT_Houston, TX

ASOs relied on gatekeepers and allies to provide insight into community preferences and expectations and advise the ASOs on best strategies for increasing reach, building rapport and trust with the community, and ultimately establishing an authentic presence within the community. This includes getting feedback (maintaining feedback loop), referrals within the community, and gaining access to peer networks' social/sexual networks. Participants suggest trusting the gatekeepers even if the information they share does not make much sense at face value.

"The main thing is, ask the population. Spend a good time trying to reach that population and try to identify key members or key leaders or key influencers, and ask them for their opinions, and then listen. Listen, that's the biggest thing, is 
listen to their opinions. When I first go to CAB and they told me to do Footlocker incentives, I was like, "Footlocker? \$25 for Footlocker aint crap. You ain't going to get a pair of shoes for $\$ 25$ at Footlocker." But because of the recommendation, we did the \$25 Footlocker gift card, and then all of a sudden there was a million people knocking on their door wanting the Footlocker incentive. I had no faith, and I was like, "That's crazy. It's \$25 for Footlocker," ... So, even if the idea sound weird or don't seem logical to you, try it out. It's really worth trying out, because everything with, okay, maybe not absolutely everything, but the majority of what they've told me, has worked out well." _-P3_Chicago, IL

Participants also relied on the $\mathrm{CAB}$ to advise them on where to go or not to go. Relying on community to identify "sacred places" and "hot spots" helps the ASOs to not encroach on the community's privacy. An outsider can easily make mistakes, like unintentionally desecrating spaces that are considered off limits for persons outside of the community, especially when that outsider assumes some level of familiarity with that community. However, with the help of an insider, as is the case for the gatekeepers and allies, mistakes like that can be avoided and the outcomes more likely to be positive for both parties. For instance, one participant found out through their $\mathrm{CAB}$ that it was unacceptable to conduct outreach in a place where sex work is practiced by transgender individuals in a community that was familiar to the participant. They said this about their CAB:

"We also bring them $[\mathrm{CAB}]$ to the table, to tell us where the hot spots are. And so, sometimes, we as researchers may feel, that we need to go to XYZ Place, not understanding that those places are a sacred place. So I used, for example, just not 
too long ago, I was talking about trans work sex work, in an area that is very familiar here in Philadelphia. And so, yes, we have access to that area, and we know that sex work happens in that area. But from our Community Advisory Board, we're probably not going to make the most connections in that area, because you're infringing upon a person's workplace, if that makes sense.”P7_Philadelphia, PA

Authentic interactions and relationships were reported as the key to sustaining meaningful connections between ASO representatives and gatekeepers/allies. To address gatekeepers' skepticisms, earn their trust, and have them motivated enough to connect the ASOs with their community, participants recommended being transparent, honest, and accountable to those individuals who are willing to be a bridge between the ASOs and the community. Transparency and honesty required ASOs to be upfront about agency goals (e.g. HIV testing goals, number of PrEP referral per year, etc.), the community's role in helping the ASO reach those goals for the good of the community, as well as the agency's capacity and capability to honor gatekeeper recommendations of best strategies for meeting community needs, etc. This strategy helped to disabuse the minds of the community members and to dispel negatively held notions that ASOs were in the community simply "to make the numbers" - to take advantage of the community to meet agency goals.

One participant shared this,

"For me it's 100\% transparency. Okay. So, you know, I think from my experience, a lot of pushback from communities about grants and programs like mine is that it tends to feel as if we use the community for a number and then 
stop. So, when I do meet with cab members, I'm 100\% transparent with grant scopes, grant numbers, and then what our intentions are. You know, my grant scopes may be test 3000 people, link 300 people to PrEP and diagnose 32 new positives. But I don't do outreach with the idea of 3000 tests, 300 you know, PrEP and 32 positives. I go out there with how do I make a difference in this community? How do I engage this community? And I really take a step back and I let them speak. I don't necessarily provide input, I just steer the conversation so that they're having an open discourse amongst themselves of how they wish or what our services to be displayed... I think that's what helps me build relationships and build trust within the community is that I truly do my best to listen to them. And then I'm also very transparent, you know, when they said, "Oh, we should have Footlocker gift codes for $\$ 50$ ". I'm transparent. I can't afford that, you know, I'm not going to say that's a good idea and move on. I'm going to say, no, we can't afford that. This is our budget. Let's talk about within the budget. You know what I mean? So I'm very transparent and, I'm very open, right? Cause I don't limit myself like other organizations do to just gift cards. Right? A lot of my organizations just do gift cards.” - P3-RT_Chicago, IL

Overall, participants demonstrated that establishing authentic presence within the community required the ASO to understand and prioritize community needs, be rooted within the community, and maximize gatekeeper/ally connections. A significant strategy for gaining access into African American communities was through the community gatekeepers and allies, as these gatekeepers/allies are the bridge between the ASO and the community. This process required giving community members a seat at the table in 
collaborative and participatory efforts that shifted power to the community, allowing them to have a say in HIV prevention efforts within their own community. Thus, being understanding of community needs, being honest, transparent, and visible in the community along with maximizing gatekeeper and ally connections are critical tools for ASOs to build rapport, engender trust among community members, and establish authentic presence within the African American community.

\section{Discussion}

AIDS service organizations play a vital role in HIV prevention, using PrEP as they are well-positioned to reach vulnerable communities through prevention and support services including promotion, education, engagement, recruitment, navigation, and evidence-based interventions that support PrEP (Centers for Disease Control and Prevention, 2020b). Yet, practice guidelines detailing strategies and best practices for ASOs to effectively tailor PrEP engagement and outreach towards African Americans a population with disproportionately high HIV vulnerability — are non-existent or have not been adequately studied and published in the literature. Developing a context-specific framework like the one presented in this study is an attempt to add to the toolbox of intervention strategies for engaging vulnerable populations (if such a toolbox already exists). This framework serves as guide for other ASOs seeking guidance on how to effectively reach African Americans with PrEP for HIV prevention with the understanding that context matters.

Participating ASO representatives articulated their experiences and lessons learned from engaging and conducting outreach with African American communities to facilitate PrEP uptake. They articulated and attempted to address the multiplicity of 
factors impacting PrEP engagement and outreach. These included individual, organizational, community, systemic, and structural level influences that conglomerate to produce various outcomes for PrEP engagement and outreach with African American communities. Their responses formed the basis of the development of the context specific framework presented in this study that was grounded in the lived experiences of ASO representatives serving African American communities.

A multi-phased framework highlighting multi-level factors impacting PrEP engagement and uptake and how to effectively navigate them in the context of outreach was appropriate, given that different factors are at play relative to engaging any individual, not just African Americans. Individual behaviors do not exist in isolation, but are rather intertwined or influenced by other factors such as interpersonal, social, economic and cultural contexts, which may restrain or promote such behaviors (DiClemente et al., 2007). Thus, interventions employing a multi-level approach tend to hold better promise in effecting behavior change in the long term (DiClemente et al., 2007). Multi-level approaches target social and economic factors, racial disparities, environmental, and political factors that may influence HIV risk (Ellen et al., 2015; Gant et al., 2014; Kahana et al., 2016; Wohlfeiler \& Ellen, 2007). Moreover, evidence suggests that multi-level/multifaceted interventions are more effective in preventing HIV (Charania et al., 2011; Jackson et al., 2012; Prado et al., 2013). Thus, this context specific framework showing the process of becoming one with the community was multi-phased with several corresponding sub-categories (although the sub-categories did not occur in any specific order). In this process framework, every phase contributed to successful PrEP engagement, outreach, and uptake, albeit in varying degrees. 
Phase one was important for acknowledging, assessing, and understanding the problem, which is a necessary first step in public health intervention development (Wight, Wimbush, Jepson, \& Doi, 2016).

During this first phase, participants reported on multiple factors that impacted PrEP engagement and outreach efforts with African American clients at high risk of HIV. The factors reported by participants were consistent with findings in the HIV and PrEP literature. Individual-level factors identified by participants included PrEP knowledge, awareness, attitudes, perceptions, fears, and conspiracy beliefs about HIV and PrEP (Collier et al., 2017; Eaton et al., 2014; Smith et al., 2012). They also identified structural and systemic barriers such as cost, lack of insurance, issues relating to low socioeconomic status (e.g., homelessness and poverty), perceived mistrust of the health care system, stigma, medical mistrust, and perceived racism. All of these influence PrEP engagement, outreach, and uptake (Arnold et al., 2014; Cahill et al., 2017; Eaton et al., 2014; Eaton, Kalichman, et al., 2017; Elopre et al., 2017; Lelutiu-Weinberger \& Golub, 2016; Wingood, Dunkle, et al., 2013). Not only did participants experience pushback from their African American clients, but they were also needed to elicit staff buy-in from within their organizations, which was difficult for some of the ASOs' participants represented. Participants reported facing pushback from some agency personnel who were not positively inclined towards biomedical prevention strategies. These individuals personally believed HIV prevention should be addressed mainly through behavioral prevention, which has been the focus of HIV prevention for decades. This finding was unsurprising as provider beliefs and attitudes have been shown to impact implementation of HIV prevention including PrEP (Owczarzak \& Dickson-Gomez, 2011). 
As participants grappled with these multiple barriers to African American engagement/outreach and their corresponding impact on ASO grant goals, participants realized they needed to find ways to overcome those challenges especially if they were going to maintain their funding to pay staff and keep their doors open. Thus, participants made the transition into phase two, as depicted by the framework. In phase two, participants devised strategies to overcome pushback and challenges experienced from clients and personnel within their respective agencies. In this phase, many participants appeared to be involved in status-quo approaches like using existing sexual health education and HIV prevention programs to address problems encountered during interactions with clients. For instance, some participants utilized incentivized HIV testing to increase PrEP referrals and evidence-based interventions (EBIs) presented in the form of workshops to increase client HIV and PrEP awareness through education. They also helped their clients navigate barriers to PrEP access like cost and insurance through MAP applications. Additionally, outside of providing sexual health services, ASOs either linked clients to other support services (like housing and food) or provided them to meet the needs their disenfranchised clients. Many of these efforts were provided one-on-one, on a case-by-case basis and, occasionally, in groups settings. These activities serve to further establish ASO's important role in the PrEP care system as delineated by the CDC: promotion and education; engagement, identification, recruitment, and EBIs supporting PrEP uptake; navigation; and directories of health and prevention services (Centers for Disease Control and Prevention, 2020b).

Further, participants saw that it was not only important to address pushback and challenges experienced from interacting with clients (the African American individuals 
and community at large), but it was expedient to also tackle (or even begin with) withinagency pushback. For any agency to successfully implement HIV prevention interventions, agency staff buy-in is paramount (Owczarzak \& Dickson-Gomez, 2011). Resource, especially personnel, are needed to get the job done, and if these personnel are not properly trained or do not endorse the organization's vision or goals about PrEP with respect to the target communities, or if the personnel have implicit or explicit biases about the target communities, the outcome of the organization's mission would be negatively impacted. Therefore, participants emphasized the importance of incorporating PrEP into the organization's philosophy as well as training staff — all staff but, more importantly, outreach personnel — in cultural humility. For African Americans specifically, trust and demeanor are key to reaching them. Due to historical and sustained mistreatment and discrimination against African Americans, perpetuated by society at large, but especially by the health care industry, African Americans have a profound mistrust of health care-related interventions (Scharff et al., 2010; Underhill et al., 2015). Thus, it befits the organization to train its staff on the history of the culture as well as cultural preferences to ensure that no additional harm is inflicted and no further damage is caused by the lack of cultural humility on the part of the agency. Thus, personnel qualities are incredibly important for effectively engaging the African American community. This community tends to trust people with whom they identify people who look like them. Participants demonstrated this as they ensured that outreach personnel characteristics were matched (to the extent possible) to the respective target client populations served. Participants reported that youth, for instance, may prefer a younger person, and gay men may be more endearing to other gay individuals or in some 
cases heterosexual women as African American women are respected and often seen as matriarchs within the culture.

While all phases of the framework contributed to successful engagement and outreach efforts with African Americans in varying degrees, ASOs reported experiencing the most success the more they operated in phase three of the framework (establishing authentic presence in community). The intensity of pushback and challenges faced by ASOs drastically decreased upon entering this phase of the framework, especially when participants demonstrated success with maximizing gatekeeper/ally connections. In general, all activities in this phase considerably contributed to establishing ASO presence in the community in an authentic way that the community saw the ASOs as part of their community. Thus, the success rate of ASOs tangibly improved as was demonstrated by reported increase in numbers of persons following through with referrals to obtain PrEP.

Phase three involves the task of building rapport/trust with the community. This required the ASO to commit to (a) prioritizing community needs and struggles, (b) being rooted in community and (c) maximizing gatekeeper/ally connections. Accomplishing these takes considerable effort and a substantial financial commitment on the part of the ASOs, who can only meet the many needs of the community to the extent that their funding allows. Unfortunately, funding presents a challenge as ASOs are grant-supported and may not always have the leeway for expenditures warranted for a comprehensive PrEP implementation that warrants extensive community stakeholder collaboration (Mayer, Chan, R, Flash, \& Krakower, 2018). Moreover, factors of socioeconomic disadvantage (such as facing food insecurity, homelessness, and lack of insurance) reportedly experienced by participants' African American clients and communities 
served have been shown to complicate HIV risk (Adimora \& Auerbach, 2010; Cene et al., 2011; Gant et al., 2014; Kahana et al., 2016; Viner et al., 2012). Further evidence suggests that mitigating these conditions of disadvantage, many of which act as determinants of HIV and other health outcomes, has great potential for lowering HIV risk.

This study has implications for practice and policy. The findings confirm previous research that multiple factors impact low PrEP uptake among African Americans including intrapersonal, socioecological, systemic, and structural factors. As this study demonstrates, improvement of PrEP uptake among African American groups with highest HIV vulnerability is contingent upon successful PrEP engagement and outreach among this population. Thus, it is imperative that ASOs who work with African American clients would consider endorsing this framework as a guide to improve their PrEP implementation efforts.

While the findings of this study demonstrated promise of ASOs to successfully improve engagement and outreach efforts among African American priority groups, it is worth noting that their success was contingent upon several conditions, many of which hinged on availability of resources such as funding that may impact agency capacity (e.g. adequacy of outreach personnel, adequacy of educational and other support services or connections to other support services, outreach personnel decision-making power). Since ASO efforts may be stifled by limited funding, organizations who provide funding to ASOs should to take this into consideration when allocating funding sources for community-related work conducted by ASOs (Yaylali et al., 2018). Policy makers should consider increasing and maintaining optimal support for health care programs, especially 
for health promotion and disease prevention programs that cater services to disenfranchised populations at increased risk of HIV. For instance, dedicated federal funding for PrEP is needed like the Ryan White HIV/AIDS program that currently focuses on HIV care and some prevention services and outreach, but not PrEP (U.S. Department of Health and Human Services, 2019). Presently, "CDC funding is limited to screening for PrEP eligibility, linkage to PrEP services, support for PrEP adherence, and increasing consumer and provider knowledge of PrEP (Yaylali et al., 2018, p. 12).” It seems counterintuitive that, on the one hand, the health care system, specifically the federal government, believes that PrEP holds the potential to end the HIV epidemic. Yet on the other hand, the very entities like ASOs who hold significant promise to increase PrEP uptake among the most vulnerable populations — the drivers of the epidemic lack the very means to do so effectively due to a paucity of dedicated federal funds for PrEP, beyond the usual HIV prevention services that allow for PrEP awareness and referrals. Even in spite of demonstrated ASOs' success in PrEP engagement and outreach efforts to vulnerable populations, PrEP access barriers such as lack of affordability and lack of insurance, complicated MAP application process, and a limited number of prescribers persist. This implies that fewer people from the most vulnerable populations will have optimal access to PrEP, thus jeopardizing the goal of ending the epidemic soon through this promising intervention.

Further, improvements in insurance policy are warranted to increase coverage for the most disadvantaged populations who are disproportionately impacted by HIV. This is particularly important as cost and affordability, due to lack of insurance and prevailing low socioeconomic status, have been shown to deter PrEP uptake among the most 
vulnerable populations who stand to benefit the most from this promising intervention (Smith et al., 2012; Wingood, Dunkle, et al., 2013) .

Finally, researcher bias during sample selection for the study was minimalized due to the appropriateness of sampling technique employed. Purposive sampling was utilized to select key informants from ASOs across the U.S. to participate in the study. Purposive sampling is advantageous for selecting persons who are experts on the topic being researched (Charmaz, 2014). A clearly defined inclusion and exclusion criteria as well as participant selection process informed by research purpose and questions helped to minimize researcher bias, which stems from subjective selection of study participants. Salazar et al. (2015) asserts that participant selection for a study should be based on the purpose of the research study which relates to the research questions.

\section{Conclusion}

ASOs play a vital role in engaging African American priority groups to improve PrEP uptake, but challenges abound. There is a dearth of exemplary strategies that guide ASOs in their PrEP engagement and outreach efforts towards African American clients. This study helps fill this gap by developing a context specific framework that serves as a guide for successfully implementing PrEP engagement and outreach with African American priority groups. A national sample of ASOs demonstrating success with implementing PrEP among African American priority groups demonstrated strategies they utilized to boost their success. The study has implications for other ASOs to improve service delivery and outreach to African American priority high-risk groups for increased PrEP engagement and uptake. More research is needed, particularly mixed method studies targeting African American priority groups — especially women and other high- 
risk heterosexuals, in addition to MSM — to more fully assess and understand the breadth and depth of multi-level factors impacting PrEP engagement, outreach, and uptake among this vulnerable population. Also, more studies should consider evaluating effectiveness of PrEP programs that cater specifically to African Americans to better allocate resources where they are most needed (Yaylali et al., 2018). Further, future studies should consider developing comprehensive national level normative guidelines for effectively implementing culturally tailored PrEP engagement and outreach with populations that demonstrate the highest HIV vulnerability, like African Americans (Mayer et al., 2018). 


\section{CHAPTER VII}

\section{DISCUSSION, IMPLICATIONS, RECOMMENDATIONS.}

\section{Discussion of Research Findings}

The overarching goal of this dissertation was to examine and address factors related to low PrEP engagement and outreach that impact PrEP uptake among African Americans in multiple priority groups in Louisville, KY. The purpose was to qualitatively explore and understand the barriers and facilitators to PrEP uptake among young (18-29 years old) African Americans residing in Louisville, Kentucky and to examine effective strategies that can be developed into recommendations for local AIDS service organizations (ASOs) to scale up PrEP outreach/delivery efforts among African American priority groups. The dissertation posed three research questions: (1) What are the multi-level barriers and facilitators to PrEP engagement, from the perspectives of African American youth groups in Louisville, KY who are at high risk for HIV; (2) What are the strategies, nationally, for PrEP outreach and delivery among various groups at high risk for HIV, particularly, African Americans, from the perspectives of key informants in ASOs across the country; and (3) How should ASOs in Louisville, KY approach PrEP outreach/delivery with African American groups at high risk to improve PrEP engagement, based on evidence from research questions 1 (local context) and 2 (national context)? Three specific aims were the focus of the dissertation study: 1) To explore and understand barriers and facilitators to, and engagement with, PrEP uptake 
among African American priority groups; (2) To develop a context specific framework grounded in experiences of ASOs of how they have successfully implemented PrEP among African American priority high-risk groups; and (3) To assess current strategies and challenges to PrEP outreach with African Americans from the perspectives of ASOs in Louisville, KY and to suggest recommendations for improving PrEP service delivery and outreach to African American priority high-risk groups.

Study findings utilized to answer research question one were reported in the two separate manuscripts presented in chapters IV and V. To address the second and third research questions, in-depth, one-on-one interviews were utilized to examine lived experiences of a national sample of key informants from ASOs across the U.S. who are successfully implementing PrEP among African American priority high-risk groups and to garner lessons learned. These findings presented in chapter VI formed the basis of developing the context specific framework — becoming one with the community - a dynamic process for successfully conducting PrEP engagement and outreach with African American priority groups. Interviews were also conducted with Louisville ASOs (submitted for presentation to APHA, 2020 and under review) (Ayangeakaa et al., 2020) to assess current strategies, challenges, and areas of improvements as a background to tailoring recommendations based on best practices from the national sample. These findings also formed the background and rationale for providing recommendations to Louisville ASOs, using the context specific framework developed from lived experiences of the national sample of ASOs and corroborated by information garnered from focus groups with a sample of Louisville African American priority groups. 
Focus groups with young African American priority groups highlighted intrapersonal or individual-level factors impacting PrEP among them as reported in chapter IV. These included PrEP awareness/knowledge, perceived HIV risk and PrEP need, fears and reservations about PrEP, and acceptability of PrEP. While there was a variation in the kind of influence these factors had on willingness to engage with and use PrEP, meaning these had both positive and negative influences of PrEP, participants reported mostly negative influences of these individual factors on PrEP use. That is, low PrEP awareness and knowledge were pervasive among participants, most were fearful of using PrEP, many (especially heterosexual individuals) did not perceive themselves as being at risk for HIV — hence not having a need for PrEP — and many had reservations about using PrEP even though they were willing to accept it as a viable option for HIV prevention. In chapter five, interpersonal, sociocultural, and systemic (structural and institutional) factors ware also reported as impacting PrEP use among African American youth in Louisville.

For the purposes of this dissertation, though, it was important to know the various types of factors influencing PrEP use among African American youth in Louisville. It was even more important to understand the reasons behind the occurrence of these factors. Thus, part of the questions posed during focus groups included reasons why participants would or would not use PrEP. Based on the responses of the focus group participants, it was observed that PrEP awareness and knowledge were low partly because the information on PrEP was not readily available or accessible within their community - the African American community. Additionally, even among the few participants who were previously aware of PrEP, there was evidence of the prevalence of 
PrEP-related stigma and conspiracy beliefs about PrEP that were born from PrEP advertisements excessively targeted towards gay individuals. Participants did not want to be associated with PrEP for fear of being perceived as being gay or having HIV. Given the high levels of stigma (Reif et al., 2014), homophobia and homonegativity within the African American community (Adimora \& Auerbach, 2010), it is no wonder that individuals in these communities would did not want to be associated with a medication that is being touted as or being perceived as being for LGBTQ+ individuals.

Furthermore, study findings revealed limited engagement between community ASOs and the Louisville community. Participants within the various priority groups indicated that ASO presence was not being felt within the African American community as many were unaware of these organizations' existence and their efforts around PrEP within the community. Priority populations provided suggestions in form of recommended for how Louisville ASOs should approach PrEP engagement and outreach with young African Americans to improve uptake.

In addition to assessing factors impacting PrEP engagement, outreach and uptake were also assessed among the Louisville priority populations. These were also assessed among both local and national ASOs catering to various African American priority populations, discussed in chapter six. These findings mirrored the focus group findings with Louisville priority groups. For instance, key informants of ASOs also reported that psychosocial, sociocultural, and systemic factors impact their prevention efforts among various priority groups within the African American community. Louisville ASOs also reported having trouble connecting with African American priority groups. This underscores the need to seek out proven strategies for accessing and building rapport with 
the African American community as demonstrated by the context specific framework developed from the perspective of national ASOs demonstrating success with PrEP engagement and outreach efforts among African Americans as presented in chapter VI.

Throughout chapter VI, findings derived from national ASOs' efforts to engage African Americans demonstrated that the key to being successful with reaching African Americans is to become one with the community, a process that was illustrated through a three-phased framework. This process showed an ongoing progression from phase I (during which ASOs are struggling to understand pushback and challenges faced both from the community and within their organization) to phase II (when they are devising means to tackle these challenges) and onto phase III (where they are building rapport and trust with the community). Throughout this process, it was evident that genuine, honest, and transparent interactions and authentic relationships were paramount to sustaining valuable engagement with African American individuals in any risk category to successfully elicit willingness to use PrEP among them.

It was evident from the framework that the more ASOs found themselves in phase III (establishing authentic presence within the community), the better the success they experienced with PrEP engagement and outreach within the African American community. Although various factors were at play in this phase, it was evident that maximizing gatekeeper and ally connections unleashed the power of the snowball effect, where trusted individuals disseminated the information to their peers or connected the ASOs to their community and social/sexual networks. ASOs elicited the help of trusted individuals and groups from the community (gatekeepers/allies) who served as a bridge between ASOs and community to help ASOs effectively implement PrEP service 
delivery and outreach within the community. This process involved a spectrum that ranged from peer referrals to ASOs identifying and training select community members from priority groups for disseminating PrEP services to community, and ensuring that community members have a seat at the table to help ASOs, in participatory efforts, to tailor interventions to the African American community. This was shown to significantly improve ASO efforts to reach target populations and to elicit PrEP uptake among them.

This ability of community-based interventions to engage individuals in initiating community-wide changes for HIV prevention is evident in the literature (Salam, Haroon, Ahmed, Das, \& Bhutta, 2014). Community-level interventions work through diffusion models (Wohlfeiler \& Ellen, 2007). That is, they produce community-wide effects by identifying and training select, well-trusted community leaders or other representatives to present HIV prevention messages back to their respective communities. For instance, in two community-level interventions (projects POL and MPowerment) conducted in the '90s, select leaders of the target audience were identified and trained to disseminate HIV prevention messages that produced effective results in decreasing HIV-risk behaviors. The intervention POL (popular opinion leaders), which identified and trained well-trusted opinion leaders in gay communities to diffuse safer sex norms among their social networks, was shown to significantly reduce any unprotected anal sex (from $36.9 \%$ to 27.5\%), multiple sex partners (by 18\%), and increase condom use during anal sex (by $16 \%$ ) among gay men in the intervention cities, compared to the comparison cities (Kelly et al., 1991). Similarly, Project Mpowerment identified and trained select members of gay communities to conduct outreach events and disseminate HIV prevention messages within their communities. This intervention too was shown to be effective in decreasing 
the proportion of men having any unprotected anal sex (from $41 \%$ to $30 \%$ ) in the intervention city, compared to no significant changes in the comparison city (Kegeles, Hays, \& Coates, 1996). More recent studies have also demonstrated the utility and effectiveness of community-level approaches in HIV prevention efforts, especially those interventions that employ participatory approaches.

Overall, this dissertation study contributes to the field of public health and fills gaps in the literature by adding to the knowledge base on HIV prevention using PrEP. A review of the literature indicated that there was a paucity of studies targeting multiple African American high-risk groups (African American women, serodiscordant couples, and other high-risk heterosexuals), besides MSM and transgender persons, and even fewer studies specifically targeting African American high-risk youth groups within these contexts (Mutchler et al., 2015). This dissertation fills those gaps and adds to the knowledge base on PrEP among African Americans by including multiple African American risk groups, not only MSM, as well as focusing on youth groups (specifically 18-29-year-olds). It highlighted multi-level factors that pose as facilitators and barriers to PrEP engagement, outreach, and use by African American priority youth populations as well as elucidated underlying root causes behind these factors. The dissertation also demonstrated the importance of addressing individual, sociocultural, and systemic level impacts on PrEP engagement and uptake as well as improving authentic connections between the African American community and ASOs. Further, the dissertation identified strategies and best practices for ASOs working with these populations to tailor interventions that address reticence to PrEP use by African American priority groups. ASOs who work with African American priority groups should glean best practices 
(illustrated in the context specific framework) derived from lived experiences of ASOs across the nation who have demonstrated success in engaging the African American community with PrEP for HIV prevention, as described in this dissertation.

\section{Limitations}

The dissertation study had some limitations and, thus, findings should be interpreted in light of these limitations. First, symbolic interactionism (SI, a philosophical underpinning of CGT), informed the development of the study guides in both the AFYA study with the various African American priority groups and the interview with the ASOs. SI posits that human beings act towards a situation (PrEP use) based on symbolic meanings (interpretations) and human interactions. However, for the AFYA

study, since a major segment of population were unaware and lacked knowledge of PrEP prior to the study, it would be fair to state that these particular participants had limited basis upon which to effectively interact with PrEP. Thus, their responses had limited prediction on what their actions would be provided that they had adequate knowledge of PrEP and had sufficient time to interact with it based on the symbolic meanings they ascribe to PrEP. On the other hand, for those participants who had prior awareness and knowledge, it can be inferred that their responses to PrEP were rooted in the symbolic meanings they ascribed to PrEP based on their existing knowledge of the intervention.

Second, TRA/TPB (the a priory theory utilized as a sensitizing concept in developing the focus group guide) posits that attitudes, norms, and perceptions influence behavior, i.e. willingness to take PrEP. However, many of my participants did not know about PrEP prior to the AFYA study. The onboarding of participants to what PrEP is during the AFYA study was the first introduction that many participants had to PrEP. 
Thus, TRA/TPB may not be in proper alignment (as a theoretical sensitizing concept) with this segment of the population in this study. On the other hand, in the case of those participants who had existing knowledge of PrEP, these theoretical sensitizing concepts were in proper alignment. That is, intention to use PrEP (willingness/unwillingness to use PrEP) was impacted either positively or negatively by attitudes towards PrEP and behavioral controls (such as adherence beliefs).

Third, not all priority high-risk groups were sufficiently represented, as some of these groups were harder to reach than others. For instance, none of the participants screened into the AFYA study identified as a person who injects drugs (PWIDs). To improve recruitment of sexual and gender minority groups, a recruiter matching the characteristics of the target demographic was hired to specifically reach MSM and LGBTQ individuals who are considered a hidden and hard-to reach population. Additionally, with the recognition that sexual and gender minority populations are highly marginalized and thus difficult-to-reach, this study utilized an incentivized method of sampling — respondent driving sampling (RDS) — that facilitates the recruitment of hard-to-reach/hidden populations. Separation of groups also did not work as well as expected because many participants fit more than one risk characteristic and several preferred to stay with their friends. Thus, we had multiple mixed groups.

Fourthly, purposive sampling was utilized to recruit ASOs into the study. Although purposive sampling is a convenience sampling approach, it is advantageous for selecting persons who are experts on the topic being researched, and it is prone to researcher bias, which stems from subjective selection of study participants. However, this limitation was taken into consideration by a clearly defined inclusion and exclusion 
criteria as well as participant selection process informed by research purpose and questions. Salazar et al. (2015) asserts that participant selection for a study should be based on the purpose of the research study which relates to the research questions.

Finally, this study reflects a local context. There was no robust PrEP promotion in this mid-southern city prior to the AFYA study and, thus, findings may differ in other contexts with more PrEP publicity.

\section{Implications}

This dissertation study was necessary, from a public health standpoint because African Americans continue to be disproportionately impacted by HIV. Despite this, there is a sparsity of research studies seeking to understand and address factors influencing PrEP uptake among various African American priority groups beyond MSM. Most reviewed studies focused mainly on MSM and bisexual males without adequately exploring other high-risk groups. It was further determined that assessing individual factors as well as structural factors influencing PrEP engagement, PrEP use, and outreach among various African American youth risk groups was warranted and expected to help identify effective strategies for improving PrEP use for HIV prevention and, ultimately, reducing disparities. Also, since most studies focus mainly on (MSM and bisexual males) without adequately exploring other high-risk groups, this study attempted to fill that gap in the literature by including youth in other high-risk categories (in addition to MSM and bisexual) who are indicated for PrEP and recommended by the CDC. Furthermore, given that African Americans in multiple priority groups (especially women, youth and MSM) are at heightened HIV risk but are not adequately engaging with PrEP, it was imperative 
to develop a deeper understanding of factors influencing PrEP among priority groups and assess strategies and best practices to improve PrEP uptake.

This dissertation study addressed the various gaps in the literature. Being the first of its kind in Kentucky, to our knowledge, this study provides baseline data for understanding multi-level factors impacting PrEP engagement, outreach, and willingness to use PrEP among African American youth (18-29 years old) residing in Louisville, KY. The study further highlights the underlying systemic root causes that are responsible for the various factors influencing PrEP engagement, outreach, and uptake among various African American priority groups. It also provides Louisville ASOs with understanding of preferred outreach methods/strategies for engaging African Americans.

Finally, the findings from this study can be extrapolated and applied to other settings with similar demographics who share similar characterizes with the African American priority groups, being cognizant of the locale context.

\section{Implications for Practice}

It is important to note that although many of these factors revealed in the study with priority African American youth groups appeared to be operating at the individual level, factors that transcend individual-level influences on behavior more heavily impose on reticence by African Americans to engage with PrEP. Thus, interventions that transcend individual level behavioral changes are warranted. In their extensive review of STD/HIV preventive interventions for adolescents, DiClemente, et al. (2007) found that individual-level interventions are insufficient for sustaining behavioral change over a prolonged period of time, whereas interventions employing a more ecological approach tend to hold more promise in effecting behavior change in the long term. They further 
asserted that individual behaviors do not exist in isolation, but are rather intertwined or influenced by other factors such as interpersonal, social, economic and cultural contexts, which may restrain or promote such behaviors.

A major contribution of the study is that findings were indicative of inadequate or ineffective PrEP promotion within the Louisville community and present a vital opportunity for tailored intervention development to address low PrEP awareness and stigmas within the community. The study indicated that current PrEP promotion may perpetuate stigmas against sexual and gender minorities and undermine risk among heterosexuals. Also, prior to the AFYA study, from which the focus group data was derived for this dissertation, there had never been a city-wide mass media PrEP campaign to create awareness within the Louisville community. This is an area of opportunity for interventionists to address influences on PrEP engagement that may be born from a lack of awareness or understanding of the purpose of PrEP for HIV prevention impacted by individual, social, and societal norms. Organizations can work with the community to mobilize them and garner community impute to tailor such interventions.

Increasing awareness and knowledge and dispelling myths and misconceptions by educating the population through mass media campaigns is a good place to start. Mass media campaigns are good examples of community-level interventions for HIV prevention and a great way to bring awareness to a community and to dispel misconceptions brought about by misinformation. These types of interventions target social, cultural, and societal norms influencing HIV risk among various target populations in a community. They take advantage of relationships among organizations and institutions (Baral et al., 2013). These types of interventions often encompass 
community mobilization, capacity-building, and coalition-building to boost prevention efforts. Several evidence-based, community-level HIV prevention interventions have been shown to produce effective behavioral changes at the community level (Centers for Disease Control and Prevention, 2017b). Evidence further demonstrates that mass media campaigns have been effective in reaching a wide audience with HIV prevention messages as well as promoting STD/HIV risk-reduction behavioral change in youth, including African American youth (Kerr, Valois, DiClemente, et al., 2015; LaCroix, Snyder, Huedo-Medina, \& Johnson, 2014; Noar et al., 2014; Romer et al., 2009; Sznitman et al., 2011). Also, culturally tailored mass media interventions (using radio and television), especially, have been shown to reduce HIV-related stigma (albeit in the short term) and increase HIV-related knowledge among African American youth (Kerr, Valois, DiClemente, et al., 2015).

Moreover, the findings from the dissertation draw considerable attention to systemic factors that impact PrEP engagement and uptake among African American priority groups. Many institutional and structural factors like availability and accessibility of PrEP (in terms of cost, affordability, and insurance) were reported to preclude willingness to use PrEP and actual PrEP uptake. Evidence suggests that for African Americans, in addition to sociocultural issues (that are typically addressed through HIV prevention education and community mobilization), socioeconomic and systemic factors are often far more important determinants of PrEP use and willingness to use PrEP than individual factors like knowledge and awareness (Eaton, Kalichman, et al., 2017). Consequently, researchers argue that structural factors (which constitute social determinants of health) such as living in disadvantaged settings, poverty, social capital, 
unemployment, and racial and ethnic segregation, to name a few, may increase vulnerability to HIV and AIDS (Holtgrave \& Crosby, 2003; Kahana et al., 2016). This argument is supported by research evidence that links HIV to social determinants of health like income, poverty, education, housing, and access to health care services (Cene et al., 2011; Gant et al., 2014; Viner et al., 2012). In this regard, interventions that target structural factors, such as those which situate individual-level risks in the context of networks, community, and public policy, hold better promise in altering the course of the HIV epidemic at population levels (Baral et al., 2013). It is important to note that even behavioral interventions like those promoting condom use, when implemented at the structural level, have been shown to be significantly effective in addressing structural issues of availability, accessibility, and acceptability in the context of condom distribution (Charania et al., 2011).

Structural-level interventions act on multiple levels to improve access to services such as improving infrastructure and transportation, which directly or indirectly influence HIV risk. For example, Project Connect Health Systems is a structural-level intervention that improved access to sexual health care services by compiling and utilizing community and health care infrastructure information to create a referral guide containing information of high-quality providers of sexual and reproductive health care services. This intervention was successful in increasing rates of HIV and STD testing among sexually active female youth (Loosier et al., 2016). Thus, intervention efforts ought to focus on addressing these structural and institutional-level factors that influence PrEP implementation among African Americans. 


\section{Implications for Policy}

It is important to note that effectively addressing structural and institutional factors impacting PrEP engagement, outreach, and uptake among African Americans is contingent upon policy at multiple levels (including organizational/institutional policy, governmental, and funding policies).

Firstly, addressing structural factors impacting PrEP within the agency through organizational policies ensured that the ASOs could adequately and effectively engage with the African American populations in ways that served to break down or mitigate PrEP-access barriers and improve quality of services for the clients/community. For instance, ASOs navigated structural barriers to PrEP implementation by helping clients access support services (food, transportation, housing/shelters, etc.) and to navigate cost and insurance-related barriers through medication assistance programs as well as helped clients apply for health coverage in some cases. This was made possible by the agency capacity such as the availability of resources and the decision-making power of the key frontline PrEP personnel for the good of the clients/priority populations served.

Second, addressing organizational/institutional factors impacting PrEP through organizational policies entailed that ASOs work to integrate PrEP into their agencies' philosophy by providing culturally tailored PrEP trainings to agency staff (including during the personnel hiring and onboarding process) to elicit staff buy-in, reduce personnel implicit and explicit biases, and improve client experiences. Some ASOs also provided training and technical assistance to affiliate and referring PrEP providers to improve provider attitudes and willingness to prescribe PrEP. 
Thirdly, an implication of this study relates to grant funding requirements for ASOs. Findings from interviews with key informants of ASOs across the country indicated that the extent to which any given ASO can successfully build rapport with the community and effectively engage in the process of becoming one with the community is contingent upon funding. Thus, strict funding requirements and the added pressure on the agency to meet funding goals (to remain funded) by reaching a specified number of targets within a stringent allotted timeframe often distracts from the quality of engagements and interactions with the community. Based on the findings from this study, the process of becoming one with the community was shown to be a precursor to successful engagement and outreach with African Americans. This process requires building rapport and engendering trust through authentic relationships and interactions with various African American priority groups, many of whom are socially marginalized by society. This takes time to accomplish. Thus, funders should consider easing funding restrictions to allow flexibility for ASOs to engage in this important process that is proven to produce better outcomes in the long run.

Fourthly, another major policy implication of this study relates to the importance of having dedicated federal funding for PrEP to improve costs associated with PrEP use (medications, every three-month doctors' visits) similar to Ryan White funding that mitigates cost-related barriers associated with HIV care (Health Resources and Services Administration, 2019b). Data from Ryan White beneficiaries indicates that approximately two-thirds of the all clients in 2018 were living under $100 \%$ of the federal poverty line (Health Resources and Services Administration, 2019a). This is likely indicative of the link between low socioeconomic factors and HIV risk. Incidentally, populations who 
stand to benefit the most from PrEP, such as African Americans in priority groups who demonstrate heightened HIV risk, are often socioeconomically disadvantaged and may have competing priorities for survival that may cause at-risk individuals to relegate their need for HIV prevention. Thus, policy makers should consider endorsing policies that serve to improve socioeconomic conditions such as homelessness, limited transportation, and food insecurity as these factors have a bearing on HIV risk as well as perceived need for PrEP.

Finally, improvements in insurance policy are warranted to increase coverage for the most disadvantaged populations who are disproportionately impacted by HIV. This is particularly important as cost and affordability, due to lack of insurance and prevailing low socioeconomic status, have been shown to deter PrEP uptake among the most vulnerable populations who stand to benefit the most from this promising intervention (Smith et al., 2012; Wingood, Dunkle, et al., 2013) .

\section{Implications for Future Research}

This dissertation study provides baseline data for understanding multi-level factors impacting PrEP engagement, outreach, and willingness to use PrEP among African American youth (18-29 years old) residing in Louisville, KY. This reflects a local context, and since there was no robust PrEP promotion in this mid-southern U.S. city prior to the AFYA study, findings may differ in other contexts with more PrEP publicity.

Additional research is needed in more African American priority groups. Future research should consider mixed-method studies targeting African American priority groups, especially women and other high-risk heterosexuals, in addition to MSM, to more 
fully assess and understand the breadth and depth of multi-level factors impacting PrEP engagement, outreach, and uptake among this vulnerable population.

Secondly, future studies should consider evaluating effectiveness of PrEP programs that cater specifically to African Americans to better allocate resources where they are needed the most (Yaylali et al., 2018). Such impact evaluation studies should focus on the outcomes of PrEP implementation specifically within the African American populations in the short term, immediate, and long term. This should provide funders with an objective way of distributing funds that is inclusive of some of the smaller ASOs located within the African American communities who have earned the trust of the community, but who may lack the capacity to secure larger, federal-level grants that allow them to scale up their PrEP outreach and engagement efforts with the African American communities.

Third, future research is needed to better understand HIV risk communication, particularly with respect to PrEP promotion or PrEP publicity. The current dissertation presented a paradox relating to current PrEP promotion. Findings revealed unintended consequences of overly targeting PrEP promotion towards MSM or LGBTQ+ populations. On the one hand, since MSM represent the highest HIV vulnerability and have historically not been adequately represented or featured in society, it makes sense that PrEP campaigns would target efforts towards the population with the highest vulnerability. On the other hand, PrEP commercials that appear to be targeted towards LGBTQ+ individuals do not resonate well with heterosexual individuals and many undermine their risk of HIV and need for PrEP as these heterosexual individuals associate PrEP with LGBTG+ individuals. These health risk communication studies 
should involve various stakeholders, particularly the various priority groups

demonstrating the highest HIV vulnerability, namely African American priority groups, to better ascertain how these populations interpret risk communication.

Fourth, more intervention studies are warranted to address medical mistrust and conspiracy theories within the African American population, particularly skepticisms regarding PrEP safety, side effects, and long-term effects born from national discourses around PrEP class action lawsuits. These interventions should involve various stakeholders within the African American community in an open and honest process from inception to finish to ensure the adequate tailoring of such interventions to the population.

Finally, the context-specific framework presented in this dissertation serves as a starting point for practice guidelines for other ASOs seeking to engage African Americans with increased HIV vulnerability. Future studies should consider developing comprehensive national-level normative guidelines based on best practices in a larger sample of ASOs demonstrating success with effectively implementing culturally tailored PrEP engagement and outreach with populations that demonstrate the highest HIV vulnerability, like African Americans (Mayer et al., 2018).

\section{Recommendations for Implementing PrEP Engagement and Outreach among African Americans in Louisville, KY.}

The findings of this study highlighted pertinent areas for ASOs to improve PrEP engagement and outreach efforts among African American priority populations in Louisville. These nine recommendations are presented in Table 6 below and are based on findings from the study with priority groups in Louisville and strategies, lessons learned, and best practices reported by ASOs across the U.S. who articulated becoming one with 
the community as a precursor to successfully engaging and conducting PrEP outreach with African Americans to improve PrEP uptake.

Table 6 Recommendations for Implementing PrEP engagement and outreach among African Americans in Louisville, KY.

\section{Suggested Action (s)}

\section{Increase ASO presence and visibility within the predominantly African American neighborhoods (West End, Louisville)}

- Have offices in areas that are more easily accessible to the community

- Show up consistently to multiple events within the community (sponsor community events if possible)

- Engage in community mobilization around overall (whole) health, which includes sexual health.

- Collaborating with the community through events

2. Have more inclusive, less stigmatizing PrEP promotion
- Include African Americans and various risk populations (LGBTQ+, heterosexual individuals, etc.)

- Pay attention to language (e.g. consider removing stigmatizing content from outreach/campaign taglines; integrate HIV prevention and PrEP into whole health promotion)

\section{Minimize PrEP access barriers}

- Provide cost/insurance navigation services to help clients address cost-related barriers.

- Provide technical assistance to affiliate PrEPprescribing providers to improve provider-client relationships

4. Increase tailored prevention education

5. Match personnel characteristics to target population
- Include statistics on various priority groups

- Hire African Americans within the community

- Train adequately (beyond entry-level skills) to ensure competency and professionalism 


\begin{tabular}{|c|c|}
\hline Recommendation & Suggested Action (s) \\
\hline & $\begin{array}{l}\text { Match age group and sexual or gender } \\
\text { characteristics to the extent possible }\end{array}$ \\
\hline $\begin{array}{l}\text { 6. Meet people where they } \\
\text { are (Utilize non- } \\
\text { traditional reach out } \\
\text { methods) }\end{array}$ & $\begin{array}{l}\text { - ASOs should explore non-traditional methods } \\
\text { like mobile vans } \\
\text { - Maximize the use of social media to enhance } \\
\text { presence }\end{array}$ \\
\hline $\begin{array}{l}\text { 7. Maximize gatekeeper/ally } \\
\text { connections }\end{array}$ & $\begin{array}{l}\text { Identify wide range of gatekeepers/allies } \\
\text { (consider popular opinion leaders, peer group } \\
\text { leaders, community leaders, faith-based leaders, } \\
\text { etc.) from community (clients, non-clients, } \\
\text { community organizations) } \\
\text { - Build authentic relationships with the } \\
\text { gatekeepers/allies (be honest and transparent } \\
\text { about agency grant goals and expectations } \\
\text { regarding PrEP) } \\
\text { Allow the community members to inform all } \\
\text { outreach efforts within the community (e.g. find } \\
\text { out from gatekeepers and allies what needs the } \\
\text { community prioritizes) }\end{array}$ \\
\hline $\begin{array}{l}\text { 8. Acknowledge, address, } \\
\text { and/or mitigate } \\
\text { sociocultural factors } \\
\text { (medical mistrust, } \\
\text { conspiracy theories, } \\
\text { stigmas) within African } \\
\text { American communities }\end{array}$ & $\begin{array}{l}\text { ASOs should invest time and resources to } \\
\text { educate and re-educate the African American } \\
\text { community to correct misinformation that breeds } \\
\text { medical mistrust, stigmas, and conspiracy } \\
\text { theories } \\
\text { - Work with various stakeholders within the } \\
\text { African American community to devise } \\
\text { strategies for open and honest communication } \\
\text { about national PrEP-related discourses (e.g. class } \\
\text { action PrEP lawsuits) that perpetuate medical } \\
\text { mistrust within the African American } \\
\text { community }\end{array}$ \\
\hline $\begin{array}{l}\text { 9. Consider having an open- } \\
\text { door policy }\end{array}$ & $\begin{array}{l}\text { Encourage community members to access ASO } \\
\text { facilities for assistance with non-sexual health } \\
\text { services } \\
\text { - Provide informal non-health related assistance } \\
\text { (e.g. provide assistance with free job } \\
\text { applications/trainings and resume' assistance) }\end{array}$ \\
\hline
\end{tabular}




\begin{tabular}{|l|l|}
\hline Recommendation & \multicolumn{1}{c|}{ Suggested Action (s) } \\
\hline & - $\begin{array}{l}\text { Provide or refer community members to other } \\
\text { support services (food, shelter, transportation } \\
\text { etc.) }\end{array}$ \\
\hline
\end{tabular}




\section{REFERENCES}

“Cisgender". (Ed.) (2019) Merriam-Webster online.

"Heterosexual". (Ed.) (2019) Merriam-Webster. Online.

"Stigma". (Ed.) (2019) Merriam-Webster. Online.

"Transgender". (Ed.) (2019) Merriam-Webster. Online.

Abad, N., Baack, B. N., O’Leary, A., Mizuno, Y., Herbst, J. H., \& Lyles, C. M. (2015). A systematic review of HIV and STI behavior change interventions for female sex workers in the United States. AIDS and Behavior, 19(9), 1701-1719.

Abara, W., Coleman, J. D., Fairchild, A., Gaddist, B., \& White, J. (2015). A faith-based community partnership to address HIV/AIDS in the southern United States: implementation, challenges, and lessons learned. J Relig Health, 54(1), 122-133. doi:10.1007/s10943-013-9789-8

Abbas, U. L. (2011). Uptake of biomedical interventions for prevention of sexually transmitted HIV. Curr Opin HIV AIDS, 6(2), 114-118. doi:10.1097/COH.0b013e328343ad7e

Adams, J. W., Lurie, M. N., King, M. R. F., Brady, K. A., Galea, S., Friedman, S. R., . . . Marshall, B. D. L. (2018). Potential drivers of HIV acquisition in AfricanAmerican women related to mass incarceration: an agent-based modelling study. BMC public health, 18(1), 1387. doi:10.1186/s12889-018-6304-x

Adams, L. M., \& Balderson, B. H. (2016). HIV providers' likelihood to prescribe preexposure prophylaxis (PrEP) for HIV prevention differs by patient type: a short report. AIDS care, 28(9), 1154-1158. doi:10.1080/09540121.2016.1153595

Adimora, A. A., \& Auerbach, J. D. (2010). Structural interventions for HIV prevention in the United States. J Acquir Immune Defic Syndr, 55(0 2), S132-135. doi:10.1097/QAI.0b013e3181fbcb38

Adimora, A. A., \& Schoenbach, V. J. (2005). Social context, sexual networks, and racial disparities in rates of sexually transmitted infections. J Infect Dis, 191(Supplement_1), S115-S122.

AIDS.gov. (2015, June 5, 2015). HIV Testing: Opt-Out Testing. Retrieved from https://www.aids.gov/hiv-aids-basics/prevention/hiv-testing/opt-out-testing/

AIDSinfo.nih.gov. (2019). HIV/AIDS Glossary.

Ajzen, I. (1991). The theory of planned behavior. Organizational behavior and human decision processes, 50(2), 179-211.

Al-Tayyib, A. A., Thrun, M. W., Haukoos, J. S., \& Walls, N. E. (2014). Knowledge of pre-exposure prophylaxis (PrEP) for HIV prevention among men who have sex with men in Denver, Colorado. AIDS Behav, 18 Suppl 3, 340-347. doi:10.1007/s10461-013-0553-6

Alegría, M., Alvarez, K., Ishikawa, R. Z., DiMarzio, K., \& McPeck, S. (2016). Removing obstacles to eliminating racial and ethnic disparities in behavioral health care.

Health Affairs, 35(6), 991-999. 
Andrew, B. J., Mullan, B. A., de Wit, J. B., Monds, L. A., Todd, J., \& Kothe, E. J. (2016). Does the theory of planned behaviour explain condom use behaviour among men who have sex with men? A meta-analytic review of the literature. AIDS and Behavior, 20(12), 2834-2844.

Arnold, E. A., Rebchook, G. M., \& Kegeles, S. M. (2014). 'Triply cursed': racism, homophobia and HIV-related stigma are barriers to regular HIV testing, treatment adherence and disclosure among young Black gay men. Cult Health Sex, 16(6), 710-722. doi:10.1080/13691058.2014.905706

Attia, S., Egger, M., Müller, M., Zwahlen, M., \& Low, N. (2009). Sexual transmission of HIV according to viral load and antiretroviral therapy: systematic review and meta-analysis. Aids, 23(11), 1397-1404.

Auerbach, J. D., Kinsky, S., Brown, G., \& Charles, V. (2015). Knowledge, attitudes, and likelihood of pre-exposure prophylaxis (PrEP) use among US women at risk of acquiring HIV. AIDS Patient Care STDS, 29(2), 102-110. doi:10.1089/apc.2014.0142

Ayala, G., Makofane, K., Santos, G.-M., Beck, J., Do, T. D., Hebert, P., . . A Arreola, S. (2013). Access to basic HIV-related services and PrEP acceptability among men who have sex with men worldwide: barriers, facilitators, and implications for combination prevention. J Sex Transm Dis, 2013.

Ayangeakaa, S. D., Kerr, J. C., Combs, R., Harris, L., Parker, K., \& Sears, J. (2020, Under review). Serving the Underserved: A Qualitative Study of U.S. AIDS Service Organizations' Lessons Learned for Conducting HIV Pre-Exposure Prophylaxis (Prep) Outreach with African American Groups. . Paper presented at the American Public Health Association (APHA) annual meeting and exposition., San Francisco, CA.

Ayangeakaa, S. D., Kerr, J. C., Combs, R., Harris, L., Sears, J., Northington, T., . . . Krigger, K. W. (2019, November 5 2019). Insider perspectives of African American high-risk groups and their recommendations to AIDS service organizations for tailoring pre-exposure prophylaxis (PrEP) outreach. Paper presented at the American Public Health Association (APHA) annual meeting and exposition., Philadelphia, PA.

Aziz, M., \& Smith, K. Y. (2011). Challenges and successes in linking HIV-infected women to care in the United States. Clinical Infectious Diseases, 52(suppl_2), S231-S237.

Baeten, J., \& McCormack, S. (2016). Welcome to the preexposure prophylaxis revolution. Curr Opin HIV AIDS, 11(1), 1-2. doi:10.1097/coh.0000000000000225

Baeten, J. M., Donnell, D., Ndase, P., Mugo, N. R., Campbell, J. D., Wangisi, J., . . . Katabira, E. (2012). Antiretroviral prophylaxis for HIV prevention in heterosexual men and women. $N$ Engl J Med, 2012(367), 399-410.

Baeten, J. M., Haberer, J. E., Liu, A. Y., \& Sista, N. (2013). Preexposure prophylaxis for HIV prevention: where have we been and where are we going? J Acquir Immune Defic Syndr, 63 Suppl 2, S122-129. doi:10.1097/QAI.0b013e3182986f69

Baral, S., Logie, C. H., Grosso, A., Wirtz, A. L., \& Beyrer, C. (2013). Modified social ecological model: a tool to guide the assessment of the risks and risk contexts of HIV epidemics. BMC public health, 13(1), 482. 
Barry, C. A. (1998). Choosing qualitative data analysis software: Atlas/ti and Nudist compared. Sociological research online, 3(3), 1-13.

Bauermeister, J. A., Meanley, S., Pingel, E., Soler, J. H., \& Harper, G. W. (2013). PrEP awareness and perceived barriers among single young men who have sex with men. Curr HIV Res, 11(7), 520-527.

Birks, M., Chapman, Y., \& Francis, K. (2008). Memoing in qualitative research: Probing data and processes. Journal of Research in Nursing, 13(1), 68-75.

Black, R. J. (1997). Animal studies of prophylaxis. The American journal of medicine, 102(5), 39-44.

Blumer, H. (1986). Symbolic interactionism: Perspective and method: Univ of California Press.

Bobashev, G. V., Zule, W. A., Osilla, K. C., Kline, T. L., \& Wechsberg, W. M. (2009). Transactional sex among men and women in the south at high risk for HIV and other STIs. Journal of Urban Health, 86(1), 32-47.

Bogart, L. M., \& Thorburn, S. (2005). Are HIV/AIDS conspiracy beliefs a barrier to HIV prevention among African Americans? JAIDS Journal of Acquired Immune Deficiency Syndromes, 38(2), 213-218.

Bourke, B. (2014). Positionality: Reflecting on the research process. The qualitative report, 19(33), 1-9.

Bradley, H., Mattson, C. L., Beer, L., Huang, P., \& Shouse, R. L. (2016). Increased antiretroviral therapy prescription and HIV viral suppression among persons receiving clinical care for HIV infection. Aids, 30(13), 2117-2124. doi:10.1097/qad.0000000000001164

Braksmajer, A., Senn, T. E., \& McMahon, J. (2016). The Potential of Pre-Exposure Prophylaxis for Women in Violent Relationships. AIDS Patient Care STDS, 30(6), 274-281. doi:10.1089/apc.2016.0098

Brawner, B. M., Reason, J. L., Goodman, B. A., Schensul, J. J., \& Guthrie, B. (2015). Multilevel drivers of human immunodeficiency virus/acquired immune deficiency syndrome among Black Philadelphians: exploration using community ethnography and geographic information systems. Nursing research, 64(2), 100110.

Buchbinder, S. P., \& Liu, A. Y. (2018). CROI 2018: Epidemic Trends and Advances in HIV Prevention. Top Antivir Med, 26(1), 1-16.

Buchbinder, S. P., \& Liu, A. Y. (2019). CROI 2019: Advances in HIV prevention and plans to end the epidemic. Top Antivir Med, 27(1), 8-25.

Buot, M. L., Docena, J. P., Ratemo, B. K., Bittner, M. J., Burlew, J. T., Nuritdinov, A. R., \& Robbins, J. R. (2014). Beyond race and place: distal sociological determinants of HIV disparities. PLoS One, 9(4), e91711. doi:10.1371/journal.pone.0091711

Burnett, J. C., Broz, D., Spiller, M. W., Wejnert, C., \& Paz-Bailey, G. (2018). HIV infection and HIV-associated behaviors among persons who inject drugs-20 cities, United States, 2015. Morbidity and Mortality Weekly Report, 67(1), 23.

Buseh, A. G., Kelber, S. T., Hewitt, J. B., Stevens, P. E., \& Park, C. G. (2006). Perceived stigma and life satisfaction: Experiences of urban African American men living with HIV/AIDS. International Journal of Men's Health, 5(1), 35.

Bush, S., Ng, L., Magnuson, D., Piontkowsky, D., \& Mera Giler, R. (2015). Significant uptake of Truvada for pre-exposure prophylaxis (PrEP) utilization in the US in 
late 2014-1Q 2015. Paper presented at the IAPAC Treatment, Prevention, and Adherence Conference.

Cahill, S., Taylor, S. W., Elsesser, S. A., Mena, L., Hickson, D., \& Mayer, K. H. (2017). Stigma, medical mistrust, and perceived racism may affect PrEP awareness and uptake in black compared to white gay and bisexual men in Jackson, Mississippi and Boston, Massachusetts. AIDS care, 1-8. doi:10.1080/09540121.2017.1300633

Calabrese, S. K., \& Underhill, K. (2015). How Stigma Surrounding the Use of HIV Preexposure Prophylaxis Undermines Prevention and Pleasure: A Call to Destigmatize "Truvada Whores". Am J Public Health, 105(10), 1960-1964. doi:10.2105/ajph.2015.302816

Cardo, D. M., Culver, D. H., Ciesielski, C. A., Srivastava, P. U., Marcus, R., Abiteboul, D., . . . McKibben, P. S. (1997). A case-control study of HIV seroconversion in health care workers after percutaneous exposure. New England Journal of Medicine, 337(21), 1485-1490.

Cardoza, V. J., Documét, P. I., Fryer, C. S., Gold, M. A., \& Butler, J. (2012). Sexual health behavior interventions for US Latino adolescents: a systematic review of the literature. Journal of pediatric and adolescent gynecology, 25(2), 136-149.

Castel, A. D., Choi, S., Dor, A., Skillicorn, J., Peterson, J., Rocha, N., \& Kharfen, M. (2015). Comparing cost-effectiveness of HIV testing strategies: targeted and routine testing in Washington, DC. PLoS One, 10(10), e 0139605.

Celum, C., \& Baeten, J. (2012). Tenofovir-based Pre-exposure prophylaxis for HIV prevention: Evidence and evolving questions. Current opinion in infectious diseases, 25(1), 51.

Cene, C. W., Akers, A. Y., Lloyd, S. W., Albritton, T., Powell Hammond, W., \& CorbieSmith, G. (2011). Understanding social capital and HIV risk in rural African American communities. J Gen Intern Med, 26(7), 737-744. doi:10.1007/s11606011-1646-4

Census.gov. (2011). The Black Population: 2010. 2010 Census Briefs. Retrieved from https://www.census.gov/prod/cen2010/briefs/c2010br-06.pdf

Centers for Disease Control and Prevention. (1995). Case-control study of HIV seroconversion in health-care workers after percutaneous exposure to HIVinfected blood--France, United Kingdom, and United States, January 1988August 1994. MMWR: Morbidity and mortality weekly report, 44(50), 929-933.

Centers for Disease Control and Prevention. (2013, January 8). Vital Signs. HIV among youth in the United States. Retrieved from http://www.cdc.gov/vitalsigns/HIVAmong Youth/index.html

Centers for Disease Control and Prevention. (2014a). HIV Surveillance Report. Centers for Disease Control and Prevention. Retrieved from http://www.cdc.gov/hiv/pdf/library/reports/surveillance/cdc-hiv-surveillancereport-2014-vol-26.pdf

Centers for Disease Control and Prevention. (2014b). Preexposure prophylaxis for the prevention of HIV infection in the United States: A clinical practice guideline. . Retrieved from https://www.cdc.gov/hiv/pdf/PrEP_fact_sheet_final.pdf 
Centers for Disease Control and Prevention. (2016a). HIV and Transgender

Communities. CDC Issue Brief. Retrieved from

https://www.cdc.gov/hiv/pdf/policies/cdc-hiv-transgender-brief.pdf

Centers for Disease Control and Prevention. (2016b). HIV Risk Among Persons Who

Exchange Sex for Money or Nonmonetary Items. Retrieved from

https://www.cdc.gov/hiv/group/sexworkers.html

Centers for Disease Control and Prevention. (2016c). Vital Signs: Trends in HIV

Diagnoses, Risk Behaviors, and Prevention Among Persons Who Inject Drugs -

United States. Retrieved from

https://www.cdc.gov/mmwr/volumes/65/wr/mm6547e1.htm

Centers for Disease Control and Prevention. (2017a). Communities in Crisis: Is There a

Generalized HIV Epidemic in Impoverished Urban Areas of the United States?

Retrieved from https://www.cdc.gov/hiv/group/poverty.html

Centers for Disease Control and Prevention. (2017b). Complete Listing of Risk

Reduction Evidence-based Behavioral Interventions. Retrieved from

https://www.cdc.gov/hiv/research/interventionresearch/compendium/rr/complete. html

Centers for Disease Control and Prevention. (2017c, January 8). HIV in the United States: At A Glance. Retrieved from

http://www.cdc.gov/hiv/statistics/overview/ataglance.html

Centers for Disease Control and Prevention. (2017d). HIV Surveillance Report. Retrieved from https://www.cdc.gov/hiv/statistics/overview/index.html

Centers for Disease Control and Prevention. (2017e). HIV Surveillance Report Retrieved from Atlanta, Georgia.: https://www.cdc.gov/hiv/statistics/overview/index.html

Centers for Disease Control and Prevention. (2017f). Pre-Exposure Prophylaxis (PrEP).

Retrieved from https://www.cdc.gov/hiv/risk/prep/

Centers for Disease Control and Prevention. (2018a). HIV Among African American Gay and Bisexual Men. Retrieved from

https://www.cdc.gov/hiv/group/msm/bmsm.html

Centers for Disease Control and Prevention. (2018b). HIV among African Americans.

Retrieved from

http://www.cdc.gov/hiv/group/racialethnic/africanamericans/index.html

Centers for Disease Control and Prevention. (2018c). HIV Among Gay and Bisexual

Men. Retrieved from https://www.cdc.gov/hiv/group/msm/index.html

Centers for Disease Control and Prevention. (2018d). HIV Among People Who Inject

Drugs. Retrieved from https://www.cdc.gov/hiv/group/hiv-idu.html

Centers for Disease Control and Prevention. (2018e). HIV Among Transgender People.

Centers for Disease Control and Prevention. (2018f). HIV Among Women Retrieved

from https://www.cdc.gov/hiv/group/gender/women/index.html

Centers for Disease Control and Prevention. (2018g, March 21). HIV among youth.

Retrieved from http://www.cdc.gov/hiv/group/age/youth/index.html

Centers for Disease Control and Prevention. (2018h, June 25, 2018). HIV in the United States by Geography. Retrieved from

https://www.cdc.gov/hiv/statistics/overview/geographicdistribution.html 
Centers for Disease Control and Prevention. (2018i, August 6, 2018). HIV in the United States: At A Glance. Retrieved from https://www.cdc.gov/hiv/statistics/overview/ataglance.html

Centers for Disease Control and Prevention. (2018j, July 23 2018). HIV/AIDS. HIV Basics. HIV Basic Statistics. Retrieved from https://www.cdc.gov/hiv/basics/statistics.html

Centers for Disease Control and Prevention. (2018k). Infectious Diseases, Opioids and Injection Drug Use. Retrieved from https://www.cdc.gov/pwid/opioid-use.html

Centers for Disease Control and Prevention. (20181, March 2018). Preexposure prophylaxis for the prevention of HIV infection in the United States-2017 update: a clinical practice guideline. Retrieved from https://www.cdc.gov/hiv/pdf/risk/prep/cdc-hiv-prep-guidelines-2017.pdf

Centers for Disease Control and Prevention. (2018m). Racial and Ethnic Disparities in Sustained Viral Suppression and Transmission Risk Potential Among Persons Receiving HIV Care - United States, 2014. Retrieved from

Centers for Disease Control and Prevention. (2018n). Vulnerable Counties and Jurisdictions Experiencing or At-Risk of Outbreaks. Retrieved from https://www.cdc.gov/pwid/vulnerable-counties-data.html

Centers for Disease Control and Prevention. (2019a, March, 19, 2019). HIV among women. Retrieved from https://www.cdc.gov/hiv/group/gender/women/index.html

Centers for Disease Control and Prevention. (2019b). HIV and African Americans. Retrieved from https://www.cdc.gov/hiv/group/racialethnic/africanamericans/index.html

Centers for Disease Control and Prevention. (2019c). HIV incidence: estimated annual infections in the U.S., 2010-2016. Retrieved from https://www.cdc.gov/nchhstp/newsroom/docs/factsheets/HIV-Incidence-FactSheet 508.pdf

Centers for Disease Control and Prevention. (2019d). HIV surveilance report, 2018 (preliminary). Retrieved from https://www.cdc.gov/hiv/library/reports/hivsurveillance.html

Centers for Disease Control and Prevention. (2019e). Statistics overview. Retrieved from https://www.cdc.gov/hiv/statistics/overview/index.html

Centers for Disease Control and Prevention. (2020a). HIV in the United States and dependent areas. Retrieved from https://www.cdc.gov/hiv/statistics/overview/ataglance.html?deliveryName=USC DC_1046-DM17205

Centers for Disease Control and Prevention. (2020b). Pre-exposure prophylaxis (PrEP). Retrieved from https://www.cdc.gov/hiv/effectiveinterventions/prevent/prep/index.html\#PrEP-and-CBOs

Charania, M. R., Crepaz, N., Guenther-Gray, C., Henny, K., Liau, A., Willis, L. A., \& Lyles, C. M. (2011). Efficacy of structural-level condom distribution interventions: a meta-analysis of US and international studies, 1998-2007. AIDS and Behavior, 15(7), 1283-1297.

Charmaz, K. (2006). Constructing grounded theory: A practical guide through qualitative analysis: Sage. 
Charmaz, K. (2014). Constructing grounded theory: sage.

Choopanya, K., Martin, M., Suntharasamai, P., Sangkum, U., Mock, P. A., Leethochawalit, M., . . . Kittimunkong, S. (2013). Antiretroviral prophylaxis for HIV infection in injecting drug users in Bangkok, Thailand (the Bangkok Tenofovir Study): a randomised, double-blind, placebo-controlled phase 3 trial. The Lancet, 381(9883), 2083-2090.

Cleary, M., Horsfall, J., \& Hayter, M. (2014). Data collection and sampling in qualitative research: does size matter? Journal of advanced nursing, 70(3), 473-475.

Cohen, J. (1960). A coefficient of agreement for nominal scales. Educational and psychological measurement, 20(1), 37-46.

Cohen, M. S., Chen, Y. Q., McCauley, M., Gamble, T., Hosseinipour, M. C., Kumarasamy, N., . . Pilotto, J. H. (2011). Prevention of HIV-1 infection with early antiretroviral therapy. New England Journal of Medicine, 365(6), 493-505.

Cohen, M. S., Smith, M. K., Muessig, K. E., Hallett, T. B., Powers, K. A., \& Kashuba, A. D. (2013). Antiretroviral treatment of HIV-1 prevents transmission of HIV-1: where do we go from here? The Lancet, 382(9903), 1515-1524.

Cohen, S. E., Liu, A. Y., Bernstein, K. T., \& Philip, S. (2013). Preparing for HIV preexposure prophylaxis: lessons learned from post-exposure prophylaxis. Am J Prev Med, 44(1 Suppl 2), S80-85. doi:10.1016/j.amepre.2012.09.036

Coleman, J. D., Tate, A. D., Gaddist, B., \& White, J. (2016). Social determinants of HIVrelated stigma in faith-based organizations. Am J Public Health, 106(3), 492-496. doi:10.2105/ajph.2015.302985

Collier, K. L., Colarossi, L. G., \& Sanders, K. (2017). Raising Awareness of PreExposure Prophylaxis (PrEP) among Women in New York City: Community and Provider Perspectives. J Health Commun, 22(3), 183-189. doi:10.1080/10810730.2016.1261969

Crepaz, N., Dong, X., Chen, M., \& Hall, H. I. (2017). Examination of HIV infection through heterosexual contact with partners who are known to be HIV infected in the United States. Aids, 31(11), 1641-1644.

Creswell, J. W. (2013). Qualitative inquiry \& research design: Choosing among five approaches (3rd ed ed.). Thousand Oaks, California: Sage

Creswell, J. W., \& Miller, D. L. (2000). Determining validity in qualitative inquiry. Theory into practice, 39(3), 124-130.

Crosby, R. A., Charnigo, R. J., Salazar, L. F., Pasternak, R., Terrell, I. W., Ricks, J., . . . Taylor, S. N. (2014). Enhancing condom use among Black male youths: A randomized controlled trial. Am J Public Health, 104(11), 2219-2225.

Dean, H. D., \& Fenton, K. A. (2010). Guest Editorial: ADDRESSING SOCIAL DETERMINANTS OF HEALTH IN THE PREVENTION AND CONTROL OF HIV/AIDS, VIRAL HEPATITIS, SEXUALLY TRANSMITTED INFECTIONS, AND TUBERCULOSIS. Public Health Reports, 1-5.

Dedoose.com. What makes Dedoose different?

Denning, P., \& DiNenno, E. (2010). Communities in crisis: is there a generalized HIV epidemic in impoverished urban areas of the United States. Paper presented at the XVIII international AIDS conference. 
DiClemente, R. J., Salazar, L. F., \& Crosby, R. A. (2007). A review of STD/HIV preventive interventions for adolescents: sustaining effects using an ecological approach. Journal of Pediatric Psychology, 32(8), 888-906.

Do, D. P., Frank, R., \& Iceland, J. (2017). Black-white metropolitan segregation and selfrated health: Investigating the role of neighborhood poverty. Social Science \& Medicine, 187, 85-92.

Dolezal, C., Frasca, T., Giguere, R., Ibitoye, M., Cranston, R. D., Febo, I., . . CarballoDieguez, A. (2015). Awareness of Post-Exposure Prophylaxis (PEP) and PreExposure Prophylaxis (PrEP) Is Low but Interest Is High Among Men Engaging in Condomless Anal Sex With Men in Boston, Pittsburgh, and San Juan. AIDS Educ Prev, 27(4), 289-297. doi:10.1521/aeap.2015.27.4.289

Dolling, D. I., Desai, M., McOwan, A., Gilson, R., Clarke, A., Fisher, M., . . Nardone, A. (2016). An analysis of baseline data from the PROUD study: an open-label randomised trial of pre-exposure prophylaxis. Trials, 17, 163. doi:10.1186/s13063-016-1286-4

Donnell, D., Baeten, J. M., Bumpus, N. N., Brantley, J., Bangsberg, D. R., Haberer, J. E., .. Hendrix, C. (2014). HIV protective efficacy and correlates of tenofovir blood concentrations in a clinical trial of PrEP for HIV prevention. Journal of acquired immune deficiency syndromes (1999), 66(3), 340.

Duncan, G. J., Ziol-Guest, K. M., \& Kalil, A. (2010). Early-childhood poverty and adult attainment, behavior, and health. Child development, 81(1), 306-325.

Dworkin, S. L., Pinto, R. M., Hunter, J., Rapkin, B., \& Remien, R. H. (2008). Keeping the spirit of community partnerships alive in the scale up of HIV/AIDS prevention: critical reflections on the roll out of DEBI (Diffusion of Effective Behavioral Interventions). Am J Community Psychol, 42(1-2), 51-59.

Eaton, L. A., Driffin, D. D., Bauermeister, J., Smith, H., \& Conway-Washington, C. (2015). Minimal Awareness and Stalled Uptake of Pre-Exposure Prophylaxis (PrEP) Among at Risk, HIV-Negative, Black Men Who Have Sex with Men. AIDS Patient Care STDS, 29(8), 423-429. doi:10.1089/apc.2014.0303

Eaton, L. A., Driffin, D. D., Kegler, C., Smith, H., Conway-Washington, C., White, D., \& Cherry, C. (2015). The role of stigma and medical mistrust in the routine health care engagement of black men who have sex with men. Am J Public Health, 105(2), e75-82. doi:10.2105/ajph.2014.302322

Eaton, L. A., Driffin, D. D., Smith, H., Conway-Washington, C., White, D., \& Cherry, C. (2014). Psychosocial factors related to willingness to use pre-exposure prophylaxis for HIV prevention among Black men who have sex with men attending a community event. Sex Health, 11(3), 244-251. doi:10.1071/sh14022

Eaton, L. A., Kalichman, S. C., Price, D., Finneran, S., Allen, A., \& Maksut, J. (2017). Stigma and Conspiracy Beliefs Related to Pre-exposure Prophylaxis (PrEP) and Interest in Using PrEP Among Black and White Men and Transgender Women Who Have Sex with Men. AIDS Behav, 21(5), 1236-1246. doi:10.1007/s10461017-1690-0

Eaton, L. A., Matthews, D. D., Driffin, D. D., Bukowski, L., Wilson, P. A., \& Stall, R. D. (2017). A Multi-US City Assessment of Awareness and Uptake of Pre-exposure Prophylaxis (PrEP) for HIV Prevention Among Black Men and Transgender Women Who Have Sex with Men. Prev Sci. doi:10.1007/s11121-017-0756-6 
Elion, R., \& Coleman, M. (2016). The preexposure prophylaxis revolution: from clinical trials to routine practice: implementation view from the USA. Curr Opin HIV AIDS, 11(1), 67-73. doi:10.1097/coh.0000000000000222

Ellen, J. M., Greenberg, L., Willard, N., Korelitz, J., Kapogiannis, B. G., Monte, D., . . . Friedman, L. B. (2015). Evaluation of the Effect of Human Immunodeficiency Virus-Related Structural Interventions: The Connect to Protect Project. JAMA pediatrics, 169(3), 256-263.

Elopre, L., Kudroff, K., Westfall, A. O., Overton, E. T., \& Mugavero, M. J. (2017). The right people, right places, and right practices: disparities in PrEP access among African American men, women and MSM in the Deep South. Journal of acquired immune deficiency syndromes (1999), 74(1), 56.

Elopre, L., McDavid, C., Brown, A., Shurbaji, S., Mugavero, M. J., \& Turan, J. M. (2018). Perceptions of HIV Pre-Exposure Prophylaxis Among Young, Black Men Who Have Sex with Men. AIDS Patient Care STDS, 32(12), 511-518. doi:10.1089/apc.2018.0121

Etikan, I. (2016). Comparison of Convenience Sampling and Purposive Sampling. American Journal of Theoretical and Applied Statistics, 5(1). doi:10.11648/j.ajtas.20160501.11

Fennie, K. P., Lutfi, K., Maddox, L. M., Lieb, S., \& Trepka, M. J. (2015). Influence of residential segregation on survival after AIDS diagnosis among non-Hispanic blacks. Annals of Epidemiology, 25(2), 113-119. e111.

Fiscella, K., \& Sanders, M. R. (2016). Racial and ethnic disparities in the quality of health care. Annual review of public health, 37, 375-394.

Flash, C. A., Dale, S. K., \& Krakower, D. S. (2017). Pre-exposure prophylaxis for HIV prevention in women: current perspectives. International journal of women's health, 9, 391.

Flash, C. A., Stone, V. E., Mitty, J. A., Mimiaga, M. J., Hall, K. T., Krakower, D., \& Mayer, K. H. (2014). Perspectives on HIV prevention among urban Black women: A potential role for HIV pre-exposure prophylaxis. AIDS patient care and STDs, 28(12), 635-642.

Fletcher, F., Ingram, L. A., Kerr, J., Buchberg, M., Bogdan-Lovis, L., \& Philpott-Jones, S. (2016). "She Told Them, Oh That Bitch Got AIDS": Experiences of Multilevel HIV/AIDS-Related Stigma Among African American Women Living with HIV/AIDS in the South. AIDS Patient Care STDS, 30(7), 349-356. doi:10.1089/apc.2016.0026

Foster, P. P., \& Gaskins, S. W. (2009). Older African Americans' management of HIV/AIDS stigma. AIDS care, 21(10), 1306-1312. doi:10.1080/09540120902803141

Gant, Z., Gant, L., Song, R., Willis, L., \& Johnson, A. S. (2014). A census tract-level examination of social determinants of health among black/African American men with diagnosed HIV infection, 2005-2009--17 US areas. PLoS One, 9(9), e107701. doi:10.1371/journal.pone.0107701

Garcia, J., Parker, C., Parker, R. G., Wilson, P. A., Philbin, M., \& Hirsch, J. S. (2016). Psychosocial Implications of Homophobia and HIV Stigma in Social Support Networks: Insights for High-Impact HIV Prevention Among Black Men Who 
Have Sex With Men. Health Educ Behav, 43(2), 217-225.

doi:10.1177/1090198115599398

Gardner, E. M., McLees, M. P., Steiner, J. F., del Rio, C., \& Burman, W. J. (2011). The spectrum of engagement in HIV care and its relevance to test-and-treat strategies for prevention of HIV infection. Clinical Infectious Diseases, 52(6), 793-800.

Gill, P., Stewart, K., Treasure, E., \& Chadwick, B. (2008). Methods of data collection in qualitative research: interviews and focus groups. British dental journal, 204(6), 291.

Glanz, K., \& Bishop, D. B. (2010). The role of behavioral science theory in development and implementation of public health interventions. Annual review of public health, 31, 399-418.

Glanz, K., Rimer, B. K., \& Viswanath, K. (2008). Health behavior and health education: theory, research, and practice (4th ed.): John Wiley \& Sons.

Glaser, B. G., \& Strauss, A. L. (1967). The discovery of grounded theory: strategies for qualitative theory. New Brunswick: Aldine Transaction.

Golafshani, N. (2003). Understanding reliability and validity in qualitative research. The qualitative report, 8(4), 597-606.

Golden, S. D., \& Earp, J. A. L. (2012). Social ecological approaches to individuals and their contexts: twenty years of health education $\&$ behavior health promotion interventions. Health Education \& Behavior, 39(3), 364-372.

Grant, R. M., Anderson, P. L., McMahan, V., Liu, A., Amico, K. R., Mehrotra, M., . . Montoya, O. (2014). Uptake of pre-exposure prophylaxis, sexual practices, and HIV incidence in men and transgender women who have sex with men: a cohort study. The Lancet infectious diseases, 14(9), 820-829.

Grant, R. M., Lama, J. R., Anderson, P. L., McMahan, V., Liu, A. Y., Vargas, L., . . . Ramirez-Cardich, M. E. (2010). Preexposure chemoprophylaxis for HIV prevention in men who have sex with men. New England Journal of Medicine, 363(27), 2587-2599.

Grant, R. M., \& Smith, D. K. (2015). Integrating Antiretroviral Strategies for Human Immunodeficiency Virus Prevention: Post- and Pre-Exposure Prophylaxis and Early Treatment. Open Forum Infect Dis, 2(4), ofv126. doi:10.1093/ofid/ofv126

Guest, G., Bunce, A., \& Johnson, L. (2006). How many interviews are enough? An experiment with data saturation and variability. Field Methods, 18(1), 59-82.

Hakre, S., Blaylock, J. M., Dawson, P., Beckett, C., Garges, E. C., Michael, N. L., . . Okulicz, J. F. (2016). Knowledge, attitudes, and beliefs about HIV pre-exposure prophylaxis among US Air Force Health Care Providers. Medicine (Baltimore), 95(32), e4511. doi:10.1097/md.0000000000004511

Hanscom, B., Janes, H. E., Guarino, P. D., Huang, Y., Brown, E. R., Chen, Y. Q., . . Donnell, D. J. (2016). Brief Report: Preventing HIV-1 Infection in Women Using Oral Preexposure Prophylaxis: A Meta-analysis of Current Evidence. J Acquir Immune Defic Syndr, 73(5), 606-608. doi:10.1097/qai.0000000000001160

Hart-Cooper, G., Irwin, C., \& Scott, H. (2018). Adolescent medicine providers' attitudes towards prescribing pre-exposure prophylaxis (PrEP) to youth at risk of HIV infection in the US: Am Acad Pediatrics.

Hatzenbuehler, M. L., Phelan, J. C., \& Link, B. G. (2013). Stigma as a fundamental cause of population health inequalities. Am J Public Health, 103(5), 813-821. 
Health Resources and Services Administration. (2018). Ryan White HIV/AIDS Program Annual Client-Level Data Report 2017. Retrieved from http://hab.hrsa.gov/data/data-reports

Health Resources and Services Administration. (2019a). Ryan White HIV/AIDS program annual client-level data report 2018. Retrieved from http://hab.hrsa.gov/data/data-reports

Health Resources and Services Administration. (2019b). Ryan White HIV/AIDS Program Client Populations and Outcomes. Retrieved from https://hab.hrsa.gov/data/datareports

Healthy People 2020. (2014). Disparities. Retrieved from https://www.healthypeople.gov/2020/about/foundation-healthmeasures/Disparities

Heckathorn, D. D. (1997). Respondent-driven sampling: a new approach to the study of hidden populations. Social problems, 44(2), 174-199.

Heitz, D. (2014). Insurers and Medicaid Cover It, So What's Behind the Slow Adoption of Truvada PrEP? Retrieved from http://www.healthline.com/health-news/hivprevention-truvada-prep-covered-by-most-insurers-050814\#1

Hennink, M. M., Kaiser, B. N., \& Marconi, V. C. (2017). Code Saturation Versus Meaning Saturation: How Many Interviews Are Enough? Qual Health Res, 27(4), 591-608. doi:10.1177/1049732316665344

Hernández-Romieu, A. C., Sullivan, P. S., Rothenberg, R., Grey, J., Luisi, N., Kelley, C. F., \& Rosenberg, E. S. (2015). Heterogeneity of HIV prevalence among the sexual networks of Black and White MSM in Atlanta: illuminating a mechanism for increased HIV risk for young Black MSM. Sex Transm Dis, 42(9), 505.

Herron, P. D. (2016). Ethical Implications of Social Stigma Associated with the Promotion and Use of Pre-Exposure Prophylaxis for HIV Prevention. LGBT Health, 3(2), 103-108. doi:10.1089/lgbt.2014.0114

Hess, K. L., Hu, X., Lansky, A., Mermin, J., \& Hall, H. I. (2017). Lifetime risk of a diagnosis of HIV infection in the United States. Annals of Epidemiology, 27(4), 238-243.

HIV.gov. (2017). U.S. Statistics. Retrieved from https://www.hiv.gov/hivbasics/overview/data-and-trends/statistics

Holtgrave, D. R., \& Crosby, R. A. (2003). Social capital, poverty, and income inequality as predictors of gonorrhoea, syphilis, chlamydia and AIDS case rates in the United States. Sexually transmitted infections, 79(1), 62-64.

Huang, Y.-1. A., Zhu, W., Smith, D. K., Harris, N., \& Hoover, K. W. (2018). HIV Preexposure Prophylaxis, by Race and Ethnicity - United States, 2014-2016. Retrieved from https://www.cdc.gov/mmwr/volumes $/ 67 / \mathrm{wr} / \mathrm{mm} 6741 \mathrm{a} 3 . \mathrm{htm}$

Jackson, C., Geddes, R., Haw, S., \& Frank, J. (2012). Interventions to prevent substance use and risky sexual behaviour in young people: a systematic review. Addiction, 107(4), 733-747.

Johnson, B. T., Scott-Sheldon, L. A., Huedo-Medina, T. B., \& Carey, M. P. (2011). Interventions to reduce sexual risk for human immunodeficiency virus in adolescents: a meta-analysis of trials, 1985-2008. Archives of pediatrics \& adolescent medicine, 165(1), 77-84. 
Kahana, S. Y., Jenkins, R. A., Bruce, D., Fernandez, M. I., Hightow-Weidman, L. B., Bauermeister, J. A., \& Adolescent Medicine Trials Network for, H. I. V. A. I. (2016). Structural Determinants of Antiretroviral Therapy Use, HIV Care Attendance, and Viral Suppression among Adolescents and Young Adults Living with HIV. PLoS One, 11(4), e0151106. doi:10.1371/journal.pone.0151106

Kalichman, S. C., \& Grebler, T. (2010). Stress and poverty predictors of treatment adherence among people with low-literacy living with HIV/AIDS. Psychosomatic medicine, 72(8), 810.

Karris, M. Y., Beekmann, S. E., Mehta, S. R., Anderson, C. M., \& Polgreen, P. M. (2014). Are we prepped for preexposure prophylaxis (PrEP)? Provider opinions on the real-world use of PrEP in the United States and Canada. Clin Infect Dis, 58(5), 704-712. doi:10.1093/cid/cit796

Kashuba, A. D., Patterson, K. B., Dumond, J. B., \& Cohen, M. S. (2012). Pre-exposure prophylaxis for HIV prevention: how to predict success. Lancet, 379(9835), 2409.

Kegeles, S. M., Hays, R. B., \& Coates, T. J. (1996). The Mpowerment Project: a community-level HIV prevention intervention for young gay men. Am J Public Health, 86(8), 1129-1136.

Kelly, J. A., Somlai, A. M., DiFranceisco, W. J., Otto-Salaj, L. L., McAuliffe, T. L., Hackl, K. L., . . . Rompa, D. (2000). Bridging the gap between the science and service of HIV prevention: transferring effective research-based HIV prevention interventions to community AIDS service providers. Am J Public Health, 90(7), 1082-1088.

Kelly, J. A., St Lawrence, J. S., Diaz, Y. E., Stevenson, L. Y., Hauth, A. C., Brasfield, T. L., . . Andrew, M. E. (1991). HIV risk behavior reduction following intervention with key opinion leaders of population: an experimental analysis. Am J Public Health, 81(2), 168-171.

Kentucky Cabinet for Health and Family Services Department for Public Health HIV/AIDS Branch. (2014). Kentucky HIV/AIDS Surveillance Report. Retrieved from http://chfs.ky.gov/nr/rdonlyres/13385b34-c9d1-42bc6-b019c0ba7a104d37/0/hivaidsannualreportjune2014.pdf

Kentucky Cabinet for Health and Family Services Department for Public Health HIV/AIDS Branch. (2016). Kentucky HIV/AIDS Surveillance Report: June 30, 2016

. Retrieved from http://chfs.ky.gov/NR/rdonlyres/DCA0FF73-C42B-41FD-AC90733939C239FD/0/AnnualReport_2016.pdf

Kentucky Cabinet for Health and Family Services Department for Public Health HIV/AIDS Branch. (2017). Kentucky HIV/AIDS Surveillance Report: June 30, 2017. Retrieved from https://chfs.ky.gov/agencies/dph/dehp/hab/Documents/Annual\%20Report\%20201 7.pdf

Kerr, J., \& Jackson, T. (2016). Stigma, sexual risks, and the war on drugs: Examining drug policy and HIV/AIDS inequities among African Americans using the Drug War HIV/AIDS Inequities Model. Int J Drug Policy, 37, 31-41. doi:10.1016/j.drugpo.2016.07.007 
Kerr, J. C., Valois, R. F., DiClemente, R. J., Carey, M. P., Stanton, B., Romer, D., . . . Fortune, T. (2015). The effects of a mass media HIV-risk reduction strategy on HIV-related stigma and knowledge among African American adolescents. AIDS Patient Care STDS, 29(3), 150-156. doi:10.1089/apc.2014.0207

Kerr, J. C., Valois, R. F., Siddiqi, A., Vanable, P., \& Carey, M. P. (2015). Neighborhood condition and geographic locale in assessing HIV/STI risk among African American adolescents. AIDS and Behavior, 19(6), 1005-1013.

Kirby, D. B., Laris, B., \& Rolleri, L. A. (2007). Sex and HIV education programs: their impact on sexual behaviors of young people throughout the world. Journal of Adolescent Health, 40(3), 206-217.

Koechlin, F. M., Fonner, V. A., Dalglish, S. L., O'Reilly, K. R., Baggaley, R., Grant, R. M., .. . Kennedy, C. E. (2017). Values and Preferences on the Use of Oral Preexposure Prophylaxis (PrEP) for HIV Prevention Among Multiple Populations: A Systematic Review of the Literature. AIDS Behav, 21(5), 1325-1335. doi:10.1007/s10461-016-1627-z

Krakower, D., Ware, N., Mitty, J. A., Maloney, K., \& Mayer, K. H. (2014). HIV providers' perceived barriers and facilitators to implementing pre-exposure prophylaxis in care settings: a qualitative study. AIDS Behav, 18(9), 1712-1721. doi:10.1007/s10461-014-0839-3

Kuhns, L. M., Hotton, A. L., Schneider, J., Garofalo, R., \& Fujimoto, K. (2017). Use of Pre-exposure Prophylaxis (PrEP) in Young Men Who Have Sex with Men is Associated with Race, Sexual Risk Behavior and Peer Network Size. AIDS Behav, 21(5), 1376-1382. doi:10.1007/s10461-017-1739-0

Kurth, A. E., Celum, C., Baeten, J. M., Vermund, S. H., \& Wasserheit, J. N. (2011). Combination HIV prevention: significance, challenges, and opportunities. Curr HIV/AIDS Rep, 8(1), 62-72.

LaCroix, J. M., Snyder, L. B., Huedo-Medina, T. B., \& Johnson, B. T. (2014). Effectiveness of mass media interventions for HIV prevention, 1986-2013: a meta-analysis. JAIDS Journal of Acquired Immune Deficiency Syndromes, 66, S329-S340.

Laufer, F. N., O'Connell, D. A., Feldman, I., \& Zucker, H. A. (2015). Vital Signs: Increased Medicaid Prescriptions for Preexposure Prophylaxis Against HIV infection--New York, 2012-2015. MMWR Morb Mortal Wkly Rep, 64(46), 12961301. doi:10.15585/mmwr.mm6446a5

Lehman, D. A., Baeten, J. M., McCoy, C. O., Weis, J. F., Peterson, D., Mbara, G., .. . Partners Pr, E. P. S. T. (2015). Risk of drug resistance among persons acquiring HIV within a randomized clinical trial of single- or dual-agent preexposure prophylaxis. J Infect Dis, 211(8), 1211-1218. doi:10.1093/infdis/jiu677

Lelutiu-Weinberger, C., \& Golub, S. A. (2016). Enhancing PrEP Access for Black and Latino Men Who Have Sex With Men. J Acquir Immune Defic Syndr, 73(5), 547555. doi:10.1097/qai.0000000000001140

Levitt, G. (2014). Are Truvada Prices An Obstacle to Americans at High Risk for HIV? Retrieved from https://www.pharmacycheckerblog.com/are-truvada-prices-anobstacle-to-americans-at-high-risk-for-hiv

Lewis, J. D. (1976). The classic American pragmatists as forerunners to symbolic interactionism. The Sociological Quarterly, 17(3), 347-359. 
Lincoln, Y. S., \& Guba, E. G. (1985). Naturalistic inquiry Newbury Park, CA: Sage

Liu, Y., Canada, K., Shi, K., \& Corrigan, P. (2012). HIV-related stigma acting as predictors of unemployment of people living with HIV/AIDS. AIDS care, 24(1), 129-135.

Loosier, P. S., Doll, S., Lepar, D., Ward, K., Gamble, G., \& Dittus, P. J. (2016). Effectiveness of an Adaptation of the Project Connect Health Systems Intervention: Youth and Clinic-Level Findings. Journal of School Health, 86(8), 595-603.

Lutfi, K., Trepka, M. J., Fennie, K. P., Ibanez, G., \& Gladwin, H. (2015). Racial residential segregation and risky sexual behavior among non-Hispanic blacks, 2006-2010. Social Science \& Medicine, 140, 95-103.

Mahajan, A. P., Sayles, J. N., Patel, V. A., Remien, R. H., Ortiz, D., Szekeres, G., \& Coates, T. J. (2008). Stigma in the HIV/AIDS epidemic: a review of the literature and recommendations for the way forward. AIDS (London, England), 22(Suppl 2), S67.

Marmot, M., Allen, J., Goldblatt, P., Boyce, T., McNeish, D., \& Grady, M. (2010). Fair society, healthy lives. Retrieved from http://www.instituteofhealthequity.org/resources-reports/fair-society-healthylives-the-marmot-review/fair-society-healthy-lives-full-report-pdf.pdf

Mayer, K. H., Chan, P. A., R, R. P., Flash, C. A., \& Krakower, D. S. (2018). Evolving Models and Ongoing Challenges for HIV Preexposure Prophylaxis Implementation in the United States. J Acquir Immune Defic Syndr, 77(2), 119127. doi:10.1097/qai.0000000000001579

Mayer, K. H., \& Venkatesh, K. K. (2010). Antiretroviral therapy as HIV prevention: status and prospects. Am J Public Health, 100(10), 1867-1876.

McCormack, S., Dunn, D. T., Desai, M., Dolling, D. I., Gafos, M., Gilson, R., . . . Schembri, G. (2016). Pre-exposure prophylaxis to prevent the acquisition of HIV1 infection (PROUD): effectiveness results from the pilot phase of a pragmatic open-label randomised trial. The Lancet, 387(10013), 53-60.

McCormack, S., Dunn, D. T., Desai, M., Dolling, D. I., Gafos, M., Gilson, R., . . . Gill, O. N. (2016). Pre-exposure prophylaxis to prevent the acquisition of HIV-1 infection (PROUD): effectiveness results from the pilot phase of a pragmatic open-label randomised trial. Lancet, 387(10013), 53-60. doi:10.1016/s01406736(15)00056-2

McCormack, S. M., Gafos, M., Desai, M., \& Cohen, M. S. (2014). Biomedical prevention: state of the science. Clinical Infectious Diseases, 59(suppl_1), S41S46.

McHugh, M. L. (2012). Interrater reliability: the kappa statistic. Biochemia medica: Biochemia medica, 22(3), 276-282.

McLeroy, K. R., Bibeau, D., Steckler, A., \& Glanz, K. (1988). An ecological perspective on health promotion programs. Health education quarterly, 15(4), 351-377.

Merriam, S. B. (2009). Qualitative Research: A Guide to Design and Implementation: Wiley.

Merriam, S. B., \& Tisdell, E. J. (2015). Qualitative research: A guide to design and implementation: John Wiley \& Sons. 
Mimiaga, M. J., Case, P., Johnson, C. V., Safren, S. A., \& Mayer, K. H. (2009). Preexposure antiretroviral prophylaxis attitudes in high-risk Boston area men who report having sex with men: limited knowledge and experience but potential for increased utilization after education. J Acquir Immune Defic Syndr, 50(1), 77-83. doi:10.1097/QAI.0b013e31818d5a27

Morris, M. W., Hong, Y.-y., Chiu, C.-y., \& Liu, Z. (2015). Normology: Integrating insights about social norms to understand cultural dynamics. Organizational behavior and human decision processes, 129, 1-13.

Morrison-Beedy, D., Carey, M. P., \& Tu, X. (2006). Accuracy of audio computerassisted self-interviewing (ACASI) and self-administered questionnaires for the assessment of sexual behavior. AIDS and Behavior, 10(5), 541-552.

Muhr, T. (1991). ATLAS/ti-A prototype for the support of text interpretation. Qualitative sociology, 14(4), 349-371.

Mullins, T. L., Zimet, G., Lally, M., \& Kahn, J. A. (2016). Adolescent Human Immunodeficiency Virus Care Providers' Attitudes Toward the Use of Oral PreExposure Prophylaxis in Youth. AIDS Patient Care STDS, 30(7), 339-348. doi:10.1089/apc.2016.0048

Mutchler, M. G., McDavitt, B., Ghani, M. A., Nogg, K., Winder, T. J., \& Soto, J. K. (2015). Getting PrEPared for HIV Prevention Navigation: Young Black Gay Men Talk About HIV Prevention in the Biomedical Era. AIDS Patient Care STDS, 29(9), 490-502. doi:10.1089/apc.2015.0002

Muvuka, B., Gray, A., Aramburu, C., Kakar, R. M., Ayangeakaa, S. D., Leslie, K. F., . . . Spencer, C. (2016). Increasing access to HIV pre-exposure prophylaxis: An assessment of physician barriers to administration. Paper presented at the 144th Annual Meeting of the American Public Health Association. , Denver, CO.

National Institute on Drug Abuse. (2018). Kentucky Opioid Summary. Retrieved from https://www.drugabuse.gov/drugs-abuse/opioids/opioid-summaries-bystate/kentucky-opioid-summary

Noar, S. M., Zimmerman, R. S., Palmgreen, P., Cupp, P. K., Floyd, B. R., \& Mehrotra, P. (2014). Development and implementation of mass media campaigns to delay sexual initiation among African American and White youth. Journal of health communication, 19(2), 152-169.

Nunn, A., Yolken, A., Cutler, B., Trooskin, S., Wilson, P., Little, S., \& Mayer, K. (2014). Geography should not be destiny: focusing HIV/AIDS implementation research and programs on microepidemics in US neighborhoods. Am J Public Health, 104(5), 775-780. doi:10.2105/ajph.2013.301864

Nyblade, L. C. (2006a). Measuring HIV stigma: existing knowledge and gaps. Psychol Health Med, 11(3), 335-345. doi:10.1080/13548500600595178

Nyblade, L. C. (2006b). Measuring HIV stigma: existing knowledge and gaps. Psychology, health \& medicine, 11(3), 335-345.

Oldenburg, C. E., Perez-Brumer, A. G., \& Reisner, S. L. (2014). Poverty matters: contextualizing the syndemic condition of psychological factors and newly diagnosed HIV infection in the United States. AIDS (London, England), 28(18), 2763. 
Onwuegbuzie, A. J., \& Leech, N. L. (2005). On becoming a pragmatic researcher: The importance of combining quantitative and qualitative research methodologies. International journal of social research methodology, 8(5), 375-387.

Otten, R. A., Smith, D. K., Adams, D. R., Pullium, J. K., Jackson, E., Kim, C. N., . . Folks, T. M. (2000). Efficacy of postexposure prophylaxis after intravaginal exposure of pig-tailed macaques to a human-derived retrovirus (human immunodeficiency virus type 2). Journal of virology, 74(20), 9771-9775.

Owczarzak, J., \& Dickson-Gomez, J. (2011). Provider perspectives on evidence-based HIV prevention interventions: barriers and facilitators to implementation. AIDS patient care and STDs, 25(3), 171-179.

Patient Access Network Foundation. (2016). HIV Prevention and Treatment. Retrieved from https://panfoundation.org/index.php/en/patients/assistance-programs/hivprevention-and-treatment

Patton, M. Q. (1999). Enhancing the quality and credibility of qualitative analysis. Health services research, 34(5 Pt 2), 1189.

Paz-Bailey, G., Noble, M., Salo, K., \& Tregear, S. J. (2016). Prevalence of HIV among US female sex workers: systematic review and meta-analysis. AIDS and Behavior, 20(10), 2318-2331.

Petroll, A. E., Staden, R. A., \& Westergaard, R. P. (2016). Pre-exposure Prophylaxis in Primary Care--A New Era in HIV Prevention. Wmj, 115(1), 6-8.

Petroll, A. E., Walsh, J. L., Owczarzak, J. L., McAuliffe, T. L., Bogart, L. M., \& Kelly, J. A. (2017). PrEP Awareness, Familiarity, Comfort, and Prescribing Experience among US Primary Care Providers and HIV Specialists. AIDS Behav, 21(5), 1256-1267. doi:10.1007/s10461-016-1625-1

Philbin, M. M., Parker, C. M., Parker, R. G., Wilson, P. A., Garcia, J., \& Hirsch, J. S. (2016). The Promise of Pre-Exposure Prophylaxis for Black Men Who Have Sex with Men: An Ecological Approach to Attitudes, Beliefs, and Barriers. AIDS Patient Care STDS, 30(6), 282-290. doi:10.1089/apc.2016.0037

Pope, C., Ziebland, S., \& Mays, N. (2000). Qualitative research in health care: analysing qualitative data. BMJ: British Medical Journal, 320(7227), 114.

Prado, G., Lightfoot, M., \& Brown, C. H. (2013). Macro-level approaches to HIV prevention among ethnic minority youth: state of the science, opportunities, and challenges. American Psychologist, 68(4), 286.

Raifman, J. R., Flynn, C., \& German, D. (2017). Healthcare Provider Contact and Preexposure Prophylaxis in Baltimore Men Who Have Sex With Men. Am J Prev Med, 52(1), 55-63. doi:10.1016/j.amepre.2016.07.031

Raphael, D. (2011). Poverty in childhood and adverse health outcomes in adulthood. Maturitas, 69(1), 22-26.

Reif, S., Safley, D., McAllaster, C., Wilson, E., \& Whetten, K. (2017). State of HIV in the US Deep South. J Community Health, 42(5), 844-853.

Reif, S. S., Whetten, K., Wilson, E. R., McAllaster, C., Pence, B. W., Legrand, S., \& Gong, W. (2014). HIV/AIDS in the Southern USA: a disproportionate epidemic. AIDS care, 26(3), 351-359. doi:10.1080/09540121.2013.824535

Reilly, K. H., Neaigus, A., Jenness, S. M., Hagan, H., Wendel, T., \& Gelpí-Acosta, C. (2013). High HIV prevalence among low-income, black women in New York 
City with self-reported HIV negative and unknown status. Journal of Women's Health, 22(9), 745-754.

Rey, D. (2011). Post-exposure prophylaxis for HIV infection. Expert review of antiinfective therapy, 9(4), 431-442.

Rich, M., \& Ginsburg, K. R. (1999). The reason and rhyme of qualitative research: why, when, and how to use qualitative methods in the study of adolescent health. Journal of Adolescent Health.

Richardson, E. T. (2014). Research on oral pre-exposure prophylaxis in sub-Saharan Africa is an example of biomedical tunnel vision. Aids, 28(10), 1537-1538.

Robinson, J., \& Yeh, E. (2011). Transactional sex as a response to risk in Western Kenya. American Economic Journal: Applied Economics, 3(1), 35-64.

Romer, D., Sznitman, S., DiClemente, R., Salazar, L. F., Vanable, P. A., Carey, M. P., . . . Stanton, B. F. (2009). Mass media as an HIV-prevention strategy: using culturally sensitive messages to reduce HIV-associated sexual behavior of at-risk African American youth. Am J Public Health, 99(12), 2150-2159.

Runyan, C. W., DeVellis, R. F., DeVellis, B. M., \& Hochbaum, G. M. (1982). Health psychology and the public health perspective: In search of the pump handle. Health Psychology, 1(2), 169.

Salam, R. A., Haroon, S., Ahmed, H. H., Das, J. K., \& Bhutta, Z. A. (2014). Impact of community-based interventions on HIV knowledge, attitudes, and transmission. Infect Dis Poverty, 3, 26. doi:10.1186/2049-9957-3-26

Salazar, L. F., Crosby, R. A., \& DiClemente, R. J. (2015). Research methods in health promotion: John Wiley \& Sons.

Sanders, G. D., Bayoumi, A. M., Sundaram, V., Bilir, S. P., Neukermans, C. P., Rydzak, C. E., . . Owens, D. K. (2005). Cost-effectiveness of screening for HIV in the era of highly active antiretroviral therapy. New England Journal of Medicine, 352(6), 570-585.

Sangaramoorthy, T., Jamison, A., \& Dyer, T. (2018). Older African Americans and the HIV Care Continuum: A Systematic Review of the Literature, 2003-2018. AIDS Behav. doi:10.1007/s10461-018-2354-4

Satcher, D., Okafor, M., \& Dill, L. J. (2012). Impact of the built environment on mental and sexual health: policy implications and recommendations. ISRN Public Health, 2012.

Scharff, D. P., Mathews, K. J., Jackson, P., Hoffsuemmer, J., Martin, E., \& Edwards, D. (2010). More than Tuskegee: understanding mistrust about research participation. J Health Care Poor Underserved, 21(3), 879-897. doi:10.1353/hpu.0.0323

Schechter, M., Do Lago, R. F., Mendelsohn, A. B., Moreira, R. I., Moulton, L. H., Harrison, L. H., \& Team, P. O. S. (2004). Behavioral impact, acceptability, and HIV incidence among homosexual men with access to postexposure chemoprophylaxis for HIV. JAIDS Journal of Acquired Immune Deficiency Syndromes, 35(5), 519-525.

Schnarrs, P. W., Gordon, D., Martin-Valenzuela, R., Sunil, T., Delgado, A. J., Glidden, D., . . . McAdams, J. (2018). Perceived social norms about oral PrEP use: differences between African-American, Latino and White gay, bisexual and other men who have sex with men in Texas. AIDS and Behavior, 22(11), 3588-3602. 
Scott, A. J., \& Wilson, R. F. (2011). Social determinants of health among African Americans in a rural community in the Deep South: an ecological exploration. Rural Remote Health, 11(1), 1634.

Seale, C. (1999). Quality in qualitative research. Qualitative inquiry, 5(4), 465-478.

Seidman, D., Carlson, K., Weber, S., Witt, J., \& Kelly, P. J. (2016). United States family planning providers' knowledge of and attitudes towards preexposure prophylaxis for HIV prevention: a national survey. Contraception, 93(5), 463-469. doi:10.1016/j.contraception.2015.12.018

Sevelius, J. M., Keatley, J., Calma, N., \& Arnold, E. (2016). 'I am not a man': Transspecific barriers and facilitators to PrEP acceptability among transgender women. Glob Public Health, 11(7-8), 1060-1075. doi:10.1080/17441692.2016.1154085

Sharpe, T. T., Voute, C., Rose, M. A., Cleveland, J., Dean, H. D., \& Fenton, K. (2012). Social determinants of HIV/AIDS and sexually transmitted diseases among black women: implications for health equity. J Womens Health (Larchmt), 21(3), 249254. doi:10.1089/jwh.2011.3350

Sherman, S. G., Hast, M., Park, J. N., Decker, M. R., Flynn, C., \& German, D. (2018). Correlates of exchange sex among a population-based sample of low-income women who have heterosexual sex in Baltimore. AIDS care, 1-9.

Sheth, A. N., Rolle, C. P., \& Gandhi, M. (2016). HIV pre-exposure prophylaxis for women. J Virus Erad, 2(3), 149-155.

Siegler, A. J., Bratcher, A., Weiss, K. M., Mouhanna, F., Ahlschlager, L., \& Sullivan, P. S. (2018). Location location location: an exploration of disparities in access to publicly listed pre-exposure prophylaxis clinics in the United States. Annals of Epidemiology. doi:10.1016/j.annepidem.2018.05.006

Siegler, A. J., Mouhanna, F., Giler-Mera, R., Yeung, H., Jones, J., Guest, J. L., . . . Sullivan, P. S. (2018). Distribution of Active PrEP Prescriptions and the PrEPto-Need Ratio, US Q2 2017. Paper presented at the Conference on Retroviruses and Opportunistic Infections (CROI), Boston, Massachusetts. http://www.croiconference.org/sites/default/files/posters2018/1430_Siegler_1022LB.pdf

Smith, D. K., Grohskopf, L. A., Black, R. J., Auerbach, J. D., Veronese, F., Struble, K. A., ... Onorato, I. M. (2005). Antiretroviral postexposure prophylaxis after sexual, injection-drug use, or other nonoccupational exposure to HIV in the United States: recommendations from the US Department of Health and Human Services. Morbidity and Mortality Weekly Report: Recommendations and Reports, 54(2), 1-20.

Smith, D. K., Maier, E., Betts, J., Gray, S., Kolodziejski, B., \& Hoover, K. W. (2016). What Community-Based HIV Prevention Organizations Say About Their Role in Biomedical HIV Prevention. AIDS Educ Prev, 28(5), 426-439. doi:10.1521/aeap.2016.28.5.426

Smith, D. K., Toledo, L., Smith, D. J., Adams, M. A., \& Rothenberg, R. (2012). Attitudes and program preferences of African-American urban young adults about preexposure prophylaxis (PrEP). AIDS Educ Prev, 24(5), 408-421. doi:10.1521/aeap.2012.24.5.408

Smith, D. K., Van, M. H., \& Huggins, R. (2017). Estimated coverage to address financial barriers to HIV preexposure prophylaxis among persons with indications for its 
use, United States, 2015. Journal of acquired immune deficiency syndromes (1999), 76(5), 465-472.

Stokols, D. (1992). Establishing and maintaining healthy environments: toward a social ecology of health promotion. American Psychologist, 47(1), 6.

Stokols, D. (1996). Translating social ecological theory into guidelines for community health promotion. American journal of health promotion, 10(4), 282-298.

Strauss, A., \& Corbin, J. (1994). Grounded theory methodology. Handbook of qualitative research, 17, 273-285.

Strauss, A., \& Corbin., J. (2015). Basics of qualitative research: Techniques and procedures for developing grounded theory (4th ed.). Thousand Oaks, CA: Sage.

Strauss, B. B., Greene, G. J., Phillips, G., 2nd, Bhatia, R., Madkins, K., Parsons, J. T., \& Mustanski, B. (2017). Exploring Patterns of Awareness and Use of HIV PreExposure Prophylaxis Among Young Men Who Have Sex with Men. AIDS Behav, 21(5), 1288-1298. doi:10.1007/s10461-016-1480-0

Sullivan, P. S., Peterson, J., Rosenberg, E. S., Kelley, C. F., Cooper, H., Vaughan, A., . . . DiClemente, R. (2014). Understanding racial HIV/STI disparities in black and white men who have sex with men: a multilevel approach. PLoS One, 9(3), e90514.

Sznitman, S., Vanable, P. A., Carey, M. P., Hennessy, M., Brown, L. K., Valois, R. F., . . . Farber, N. (2011). Using culturally sensitive media messages to reduce HIVassociated sexual behavior in high-risk African American adolescents: results from a randomized trial. Journal of Adolescent Health, 49(3), 244-251.

Tracy, S. J. (2010). Qualitative Quality: Eight "Big-Tent" Criteria for Excellent Qualitative Research. Qualitative inquiry, 16(10), 837-851. doi:10.1177/1077800410383121

Trotter II, R. T. (2012). Qualitative research sample design and sample size: Resolving and unresolved issues and inferential imperatives. Preventive medicine, 55(5), 398-400.

U.S. Department of Health and Human Services. (2019). About the Ryan White HIV/AIDS Program. Retrieved from https://hab.hrsa.gov/about-ryan-whitehivaids-program/about-ryan-white-hivaids-program

U.S. Food and Drug Administration. (2012). News \& Events. Retrieved from https://wayback.archiveit.org/7993/20161022204837/http://www.fda.gov/NewsEvents/Newsroom/PressA nnouncements/ucm 312210.htm

U.S. Food and Drug Administration. (2019). FDA News Release. Retrieved from https://www.fda.gov/news-events/press-announcements/fda-approves-seconddrug-prevent-hiv-infection-part-ongoing-efforts-end-hiv-epidemic

Underhill, K., Morrow, K. M., Colleran, C., Holcomb, R., Calabrese, S. K., Operario, D., ... Mayer, K. H. (2015). A Qualitative Study of Medical Mistrust, Perceived Discrimination, and Risk Behavior Disclosure to Clinicians by U.S. Male Sex Workers and Other Men Who Have Sex with Men: Implications for Biomedical HIV Prevention. J Urban Health, 92(4), 667-686. doi:10.1007/s11524-015-99614

Ungar, L. (2017). Kentucky's Appalachian hills are a shared drug needle away from the next big HIV outbreak. Louisville Courier Journal. 
United States Census Bureau. (2016). Poverty Status in The Past 12 Months 2012-2016 American Community Survey 5-Year Estimates. Retrieved from https://factfinder.census.gov/faces/tableservices/jsf/pages/productview.xhtml?src= $\underline{\text { bkmk }}$

United States Census Bureau. (2017). Income and Poverty in the United States: 2016. Retrieved from https://www.census.gov/library/publications/2017/demo/p60259.html

Upchurch, D. M., Mason, W. M., Kusunoki, Y., \& Kriechbaum, M. J. (2004). Social and Behavioral Determinants of Self-Reported STD Among Adolescents. Perspectives on Sexual and Reproductive Health, 36(6), 276-287.

US Food and Drug Administration. (2012). Background package for NDA 21752/Supplement 30. Retrieved from http://www.fda.gov/downloads/AdvisoryCommittees/CommitteesMeetingMateria 1s/Drugs/AntiviralDrugsAdvisoryCommittee/UCM303213.pdf

USAID.gov. HIV prevention for serodiscordant couples. Retrieved from https://aidsfree.usaid.gov/resources/pkb/combination/hiv-preventionserodiscordant-couples

Van Handel, M., Kann, L., Olsen, E. O. M., \& Dietz, P. (2016). HIV testing among US high school students and young adults. Pediatrics, 137(2), e20152700.

Viner, R. M., Ozer, E. M., Denny, S., Marmot, M., Resnick, M., Fatusi, A., \& Currie, C. (2012). Adolescence and the social determinants of health. The Lancet, 379(9826), 1641-1652.

Vissman, A. T., Hergenrather, K. C., Rojas, G., Langdon, S. E., Wilkin, A. M., \& Rhodes, S. D. (2011). Applying the theory of planned behavior to explore HAART adherence among HIV-positive immigrant Latinos: elicitation interview results. Patient Education and Counseling, 85(3), 454-460.

Volk, J. E., Marcus, J. L., Phengrasamy, T., Blechinger, D., Nguyen, D. P., Follansbee, S., \& Hare, C. B. (2015). No new HIV infections with increasing use of HIV preexposure prophylaxis in a clinical practice setting. Clinical Infectious Diseases, 61(10), 1601-1603.

Volunteers of America. (2018). Learn More About PREP, The HIV Prevention Pill. Retrieved from https://www.voamid.org/prep

Wagstaff, A. (2002). Poverty and health sector inequalities. Bulletin of the world health organization, 80, 97-105.

White House Office of National AIDS Policy. (2010, July 13, 2010). National HIV/AIDS strategy for the United States Retrieved from https://search.archives.gov/search?query=National+AIDS+strategy\&op=Search\& affiliate $=$ obamawhitehouse

Wight, D., Wimbush, E., Jepson, R., \& Doi, L. (2016). Six steps in quality intervention development (6SQuID). J Epidemiol Community Health, 70(5), 520-525. doi:10.1136/jech-2015-205952

Willard, N., Chutuape, K., Stines, S., Ellen, J. M., \& Interventions, A. M. T. N. f. H. A. (2012). Bridging the gap between individual-level risk for HIV and structural determinants: Using root cause analysis in strategic planning. Journal of prevention \& intervention in the community, 40(2), 103-117. 
Wingood, G. M., Dunkle, K., Camp, C., Patel, S., Painter, J. E., Rubtsova, A., \& DiClemente, R. J. (2013). Racial differences and correlates of potential adoption of pre-exposure prophylaxis (PrEP): results of a national survey. Journal of acquired immune deficiency syndromes (1999), 63(0 1), S95.

Wingood, G. M., Rubtsova, A., DiClemente, R. J., Metzger, D., \& Blank, M. (2013). A New Paradigm for Optimizing HIV Intervention Synergy The Role of Interdependence in Integrating HIV Prevention Interventions. Journal of acquired immune deficiency syndromes (1999), 63(0 1), S108.

Wohlfeiler, D., \& Ellen, J. M. (2007). The limits of behavioral interventions for HIV prevention. Prevention is primary: strategies for community well-being. San Francisco, CA: Jossey-Bass, 329-347.

World Health Organization. (2016). Social Determinants of Health. Retrieved from http://www.who.int/social_determinants/en/

Yardley, L. (2000). Dilemmas in qualitative health research. Psychology and health, 15(2), 215-228.

Yaylali, E., Farnham, P. G., Cohen, S., Purcell, D. W., Hauck, H., \& Sansom, S. L. (2018). Optimal allocation of HIV prevention funds for state health departments. PLoS One, 13(5), e0197421. doi:10.1371/journal.pone.0197421

Zanoni, B. C., \& Mayer, K. H. (2014). The adolescent and young adult HIV cascade of care in the United States: exaggerated health disparities. AIDS patient care and STDs, 28(3), 128-135.

Ziol-Guest, K. M., Duncan, G. J., Kalil, A., \& Boyce, W. T. (2012). Early childhood poverty, immune-mediated disease processes, and adult productivity. Proceedings of the National Academy of Sciences, 109(Supplement 2), 17289-17293. 


\title{
APPENDICES
}

\section{Appendix A: Focus Group Guide}

\author{
UofL Institutional Review Boards \\ IRB NUMBER: 18.0020 \\ IRB APPROVAL DATE: 02/25/2018
}

\section{Focus Group Guide}

1. Have you ever heard of PrEP (pre-exposure prophylaxis), a daily medication that can prevent people from becoming HIV positive?

Probe:

a) Where did you hear about PrEP? Doctor, friends, family, social media, television, etc.?

2. What do you think about PrEP?

3. Would you consider getting PrEP yourself? Why or why not?

4. Can you think of any reasons why young people would or would not want to get PrEP? Probe:

5. Can you describe any barriers to obtaining PrEP, specifically within your communities?

6. What would make it easier for people at risk for HIV to get PrEP?

7. An AIDS service organization helps provide HIV prevention education to the community as well as care and support for people living with HIV. Examples include Volunteers of America, the House of Ruth etc.

How can AIDS service organizations (ASOs) break down barriers to getting PrEP?

Probe:

a) What are some best strategies that ASOs can use for promoting PrEP use among young people?

8. Health communication campaigns give the public information about how to stay healthy and avoid disease.

What should a campaign about PrEP look like and sound like?

Probe:

a) If you have seen a campaign on PrEP in the past, what worked and what did not work?

b) What messages about HIV and PrEP should be included?

c) What messages about HIV and PrEP should be avoided or left out? 


\title{
Appendix B: ASO Recruitment Email
}

UofL Institutional Review Boards IRB NUMBER: 18.0020

IRB APPROVAL DATE: 09/21/2019

\author{
Recruitment Email
}

Hello

My name is Suur Ayangeakaa. I am an African American cis-gender female and a doctoral student from the university of Louisville, Kentucky.

I am currently working on my dissertation which focuses on understanding factors impacting low PrEP uptake among African Americans. My study aims are

1. To explore and understand barriers and facilitators to, and engagement with, PrEP uptake among priority high-risk groups.

2. To identify effective strategies for AIDS service organizations to improve service delivery and outreach to priority high-risk groups for PrEP engagement.

My objective is to reach out to AIDS service organizations like yours who have been successful with PrEP initiatives among African Americans, who have been involved in PrEP activities for at least 1 year (including PrEP education), have an established and robust infrastructure for PrEP outreach and/or delivery to African Americans (especially groups considered high-risk), and have had success with client uptake.

I am reaching out to you because I need your help completing my dissertation, which I hope would help improve PrEP uptake in our community in Louisville. My goal is to gain some insight into you experiences catering to African Americans and what factors have contributed to your success. This information will be useful in aiding the work of AIDS service organizations in Louisville to improve PrEP outreach to African American groups.

It would be a great honor to speak with a representative from your organization who is an expert on your PrEP initiative.

I sincerely appreciate your willingness to assist with this endeavor.

Sincerely,

Suur. 


\section{Appendix C: National ASO Interview Guide}

\section{Revised Interview Guide for National ASOs}

Section 1: Description of Organization

1. Gender (pronouns) of representative, location, and year organization was established.

2. What types of HIV prevention services does your organization provide?

3. How long has your organization been providing HIV services? PrEP services?

Section 2: Description of PrEP delivery and outreach activities provided by the organization

4. What has implementing PrEP delivery (or outreach or both) meant to your organization?

5. Can you describe the population that you cater PrEP services to?

Probe:

- Which specific priority groups do you target for PrEP services? MSM, Women, PWIDs, high-risk heterosexuals (serodiscordant couples and persons who do not use condoms or have partners with unknown HIV status)?

- What age groups do you target?

6) Can you please describe your experience with providing PrEP services to African American groups?

Probe:

- What is your strategy for locating the various African American priority groups (MSM, women, PWIDs, high-risk heterosexuals [serodiscordant couples and persons who do not use condoms or have partners with unknown HIV status])?

- In your experience, how does each of the different groups prefer to be approached?

- Please describe your experiences with reaching youth and young adults (specifically, 18-29 year olds). 


\section{Appendix D: Louisville ASO Interview Guide}

UofL Institutional Review Boards

IRB NUMBER: 18.0020

IRB APPROVAL DATE: 09/21/2019

\section{Interview Guide for Louisville ASOs}

Section 1: Description of Organization

1. Gender (pronouns) of representative, location, and year organization was established.

2. What types of HIV prevention services does your organization provide?

3. How long has your organization been providing HIV services? PrEP Services?

Section 2: Description of HIV prevention activities provided by the organization

4. What does HIV prevention look like for you as an organization?

Probe:

- What types of activities do you engage in for HIV prevention?

5. What has implementing PrEP delivery (or outreach or both) meant to your organization?

6. Can you describe the population that you cater HIV prevention (PrEP) services to?

- Which specific high-risk (priority) groups do you target? MSM, Women, PWIDs, high-risk heterosexuals (serodiscordant couples and persons who do not use condoms or have partners with unknown HIV status)?

- What age groups do you target?

7. Can you please describe your experience with providing HIV prevention (PrEP) services to African Americans?

Probe:

- What is your strategy for locating the various African American target groups (MSM, women, PWIDs, high-risk heterosexuals [serodiscordant couples and persons who do not use condoms or have partners with unknown HIV status])?

- In your experience, how does each of the different groups prefer to be approached? 


\section{Appendix E: ASO Preamble Consent}

UofL Institutional Review Boards

IRB NUMBER: 18.0020

IRB APPROVAL DATE: 10/14/2019

Study Title: Increasing Pre-exposure Prophylaxis among High-risk African Americans in Louisville, KY.

\section{Subject Preamble Consent Document and Research Authorization}

Date:

\section{Background}

You are being invited to participate in a research study titled, Increasing Pre-exposure Prophylaxis (PrEP) among High-risk African Americans in Louisville, KY. The study is being conducted by Dr. Jelani Kerr, Dr. Ryan Combs, and Suur Ayangeakaa. The study is sponsored by the Jewish Heritage Fund for Excellence and the University of Louisville, Department of Health Promotion and Behavioral Sciences. You are being asked to provide responses to interview questions to help us know more about your PrEP efforts. The interview will take approximately 60 minutes. You may be asked to participate in a subsequent interview. There are no known risks for your participation in this research study. The information collected may not benefit you directly. The information learned in this study may be helpful for improving PrEP efforts among African Americans in Louisville, KY.

\section{Confidentiality}

Total privacy cannot be guaranteed. We will protect your privacy to the extent permitted by law. You will be given a unique identification number, and this will be recorded on the interview data instead of your name. Several measures will help ensure the safety of your data. The data will be collected on an audio recorder, transported to a computer using an encrypted jump drive, and stored on a secure, password-protected location of the University of Louisville's server. Only the research team and staff members that deal with data will have access to these records. If the results of the study are published, your name will not be made public.

While unlikely, the following may look at the study record: The University of Louisville Institutional Review Board, Human Subjects Protection Program Office, and Privacy Office.

\section{Voluntary Participation}

Taking part in this study is voluntary. By answering interview questions, you agree to take part in this research study. You do not have to answer any questions that make you uncomfortable. You may choose not to take part at all. If you decide to be in this study you may stop taking part at any time. If you decide not to be in this study or if you stop taking part at any time, there will be no penalty.

\section{Research Subject's Rights, Questions, Concerns, and Complaints}

If you have any questions, concerns, or complaints about the research study, please contact: Dr. Jelani 


\section{Appendix F: Study Outcome Letter}

\section{unvensistr of LOUISVILLE}

Human Subjects Protection Program Office

MedCenter One - Suite 200

501 E. Broadway

Louisville, KY 40202-1798

Office: 502.852.5188 Fax: 502.852.2164

\section{DATE:}

TO:

IRB NUMBER:

STUDY TITLE:

REFERENCE \#:

IRB STAFF CONTACT:
September 23, 2019

Jelani C Kerr

18.0020

Increasing Pre-exposure Prophylaxis among High-risk African Americans in Louisville, KY 693754

Barbara Dearinger 852-5987 badear01@louisville.edu

The amendment request has been received by the Human Subjects Protection Program Office and approved by the Chair/Vice Chair of the Institutional Review Board (IRB) on 09/21/2019 through the expedited review procedure according to 45 CFR 46.110(B). The following documents have been reviewed and approved:

\begin{tabular}{|l|l|l|}
\hline Title & Version Date & Outcome \\
\hline Key informant interview guide Louisville & $09 / 12 / 2019$ & Approved \\
\hline Recruitment Email & $09 / 12 / 2019$ & Approved \\
\hline Key informant interview guide & $04 / 29 / 2019$ & Approved \\
\hline
\end{tabular}

The modifications include modifying this application by adding a recruitment email for AIDS service organizations. Also interviewing AIDS service organizations within Louisville, Kentucky whereas before only interviewing representatives outside of Louisville.

Please begin using your newly stamped approved documents at this time. The committee will be advised of this action at a regularly scheduled meeting.

For guidance on using iRIS, including finding your approved stamped documents, please follow the instructions at http://louisville.edu/research/humansubjects/iRISSubmissionManual.pdf.

If you have any questions, please contact the IRB analyst listed above or the Human Subjects Protection Program office at hsppofc@louisville.edu.

We value your feedback. Please let us know how you think we are doing: https://www.surveymonkey.com/r/CCLHXRP

Sincerely,

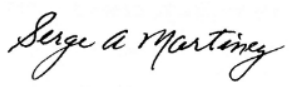

Serge A. Martinez, M.D., J.D. Vice Chair

Biomedical Institutional Review Board 


\title{
CURRICULUM VITA
}

\author{
Suur D. Ayangeakaa \\ 1206 Stonewood Court \\ Annapolis, MD 21409 \\ $+1-574-310-4362$ \\ suur.ayangeakaa@louisville.edu
}

\begin{abstract}
INTERESTS
Global Health Initiatives and Community Empowerment Programs, Women and Children Nutrition, Community-Based Interventions for Nutrition, Health, and Wellness. Health Promotion, Behavioral and Structural Interventions, Social Determinants of Health, Chronic and Infectious Disease Prevention, Sexually Transmitted Diseases (STI), Viral Hepatitis, and HIV/AIDS Disparities among Minority and Underserved Populations.
\end{abstract}

\section{EDUCATION}

Doctor of Philosophy (Ph.D.), Public Health Sciences. G.P.A.: 3.98/4.0 (May 2020)

- Concentration: Health Promotion

- School: University of Louisville, School of Public Health and Information Sciences, Louisville, Kentucky, U.S.A

Master of Public Health (MPH). G.P.A.: 4.0/4.0 (May 2013)

- Concentration: Health Promotion and Disease Prevention

- Capstone Project: HIV/AIDS and Hepatitis C Among African Americans: Strategies for increasing screening, awareness, and counseling for specific target groups.

- School: Chicago State University, Chicago, Illinois, U.S.A

Bachelor of Arts Degree (B.A.). G.P.A.: 3.54/4.0 (May 2009)

- Major: Chemistry (Pre-Med)

- School: Dordt College, Sioux Center, Iowa, U.S.A

\section{TeChNICAL SKILLS}

Computer Literacy: Microsoft Word, Excel, PowerPoint, Statistical Program for Social Sciences (SPSS), Atlas.ti, Dedoose and working knowledge of NVivo qualitative software. 


\section{RESEARCH EXPERIENCE}

Graduate Research Assistant/Doctoral Student Investigator (2015-2020)

Institution: School of Public Health and Information Sciences, University of Louisville, Kentucky.

- Dissertation study through Department of Health Promotion and Behavioral Sciences:

Designed study focused on (1) assessing and addressing multi-level factors impacting low uptake of HIV pre-exposure prophylaxis (PrEP) among African American priority groups: men who have sex with Men, LGBTQ+ and high-risk heterosexual men and women (commercial sex workers and serodiscordant heterosexual couples as well as other heterosexual persons who do not use condoms or have partners with unknown HIV status) (2) assessing strategies for AIDS service organizations (ASOs) to scale up PrEP outreach among African American priority groups.

- Successfully conducted participant recruitment.

- Facilitated focus groups with priority populations and one-on-one interviews with national and local ASOs.

- Conducted literature reviews, manuscript preparation, conference presentations.

\section{Multi-faceted PrEP intervention project through Department of Health Promotion and Behavioral Sciences:}

- Contributed to PrEP multi-media campaign development, implementation, evaluation through recruiting study participants, distributing PrEP promotion materials at community events, participating in content development for social media campaign, participating in formative research process for tailoring campaign messaging to target audience, conducting baseline and post-campaign data collection for evaluation.

- Provided research supported through focus group facilitation, quantitative survey administration via Audio Computer Assisted Self Interviews (ACASI), data management, and analysis.

- Conducted literature reviews, manuscript preparation, and oral presentation of findings.

\section{HIV/AIDS Prevention Research with various high-risk groups through Department of Health Promotion and Behavioral Sciences:}

- Contributed to investigation of ex-offender stigma, HIV-related stigma, and care continuum participation among African American HIV positive ex-offender's HIVrelated stigma.

- Administered ACASI surveys, conducted data management and analysis.

- ConductedsecondarydataanalysisonHIVpreventionstudyamongAfricanCaribbean, Black youth in North America, co-authored a published peer-reviewed journal article. 


\section{Health Literacy/ Insurance Literacy Research Intervention through Commonwealth Institute of Kentucky-Office of Public Health Practice:}

- Co-conducted a study on examining the development, use, applicability, and design of health insurance, health systems, and health behavior literacy materials to residents of West Louisville.

- Conducted participant recruitment, data collection, facilitated focus groups.

- Completed data analysis, literature reviews, manuscript preparation, presentations, co-authored peer-reviewed journal article.

\section{Community-Based Participatory Research [CBPR] through Commonwealth} Institute of Kentucky-Office of Public Health Practice:

- Utilized an innovative CBPR technique, Boot Camp Translation (BCT), to develop tailored health communication campaign addressing depression among West Louisville residents.

- Participated in community advisory meetings to engage key community stakeholders and to forge academic-community partnerships.

Co-Investigator Empowerment-based HIV prevention for previously incarcerated women: (2012-2013)

Institution: HIV/AIDS Research and Policy Institute, Chicago State University Contribution:

- Administered individual interviews, transcribed transcripts.

- Utilized findings to inform HIV prevention among former female prison inmates.

Co-Investigator: Prevalence of tattoo recipients among college students, and their knowledge, awareness and practices to Hepatitis C virus (2012-2013)

Institution: Health Studies Department, Chicago State University. Contribution:

- Completed survey questionnaire development, participant recruitment, data collection and analysis using SPSS (Quantitative software).

- Conducted literature reviews, manuscript preparation, and oral presentation of findings.

\section{TEACHING EXPERIENCE}

Graduate Teaching Assistant, University of Louisville, Kentucky (Spring, 2020)

- Qualitative Research Methods in Public Health (PHPB 711-Graduate Course).

- Assisted instructor, co-lectured some class sections.

- Facilitated interactive class activities with students.

- Trained students in data collection techniques, specifically focus group facilitation.

Graduate Research and Teaching Assistant, Chicago State University, Chicago, Illinois (2012 - 2013)

- Provided instruction to 11 senior level Health Information Administration students in quantitative analysis using SPSS, graded SPSS lab assignments, compiled and reported final grades.

- Pioneered, developed, and evaluated a seminar aimed at educating undergraduate students about graduate school application process. 
- Served as peer mentor to undergraduate students.

- Conducted prep sessions for graduate record exam (GRE).

Undergraduate Teaching Assistant, Dordt College Sioux Center, Iowa (2007-2009)

- Provided tutorial services to students enrolled in organic chemistry.

- Set up and supervised lab experiments.

- Prepared lab reagents for experiment.

- Created and graded lab quizzes, weekly reports, and homework assignments.

\section{INVITED TALKS}

Panelist: School of Public Health \& Information Sciences Preview Day (February, 2020) Institution: University of Louisville, Louisville, KY

Guest Speaker: Health Talk (December 2019)

Institution: Fifth Street Baptist Church, Louisville, Kentucky.

Topic: Emphasizing the importance of health and wellness in the Church.

\section{Guest Lecture: Social Determinants of Health (PHPB 630-Graduate Course)}

(December 2019)

Institution: School of Public Health and Information Sciences, University of Louisville, Kentucky.

Topic: Exploring and addressing factors impacting low pre-exposure prophylaxis (PrEP) engagement and uptake among young, urban African Americans in high risk groups.

Speaker: Women's HIV Pre-Exposure Propylaxis (PrEP) Summit (June 2017) Institution: University of Louisville, Kentucky.

Topic: Examining trends in HIV prevalence, incidence, and disparities across Kentucky.

\section{Conference Presentations}

147th APHA Annual Meeting and Exposition (November, 2019)

Philadelphia, Pennsylvania, U.S.A

- Authors: Suur D Ayangeakaa, Jelani Kerr, Ryan Combs, Lesley Harris, Jeanelle Sears, Toya Northington, Kimberly Parker, Emma Sterrett-Hong, and Karen Krigger.

- Round Table Title: Individual and Structural Factors Influencing Low Pre-Exposure Prophylaxis (Prep) Engagement and Uptake Among Young, Urban African Americans In High Risk Groups.

147th APHA Annual Meeting and Exposition (November, 2019)

Philadelphia, Pennsylvania, U.S.A

- Authors: Suur D Ayangeakaa, Jelani Kerr, Ryan Combs, Lesley Harris, Jeanelle Sears, Toya Northington, Kimberly Parker, Emma Sterrett-Hong, and Karen Krigger. 
- Round Table Title: Insider Perspectives of African American High-Risk Groups and their Recommendations to AIDS Service Organizations for Tailoring Pre-Exposure Prophylaxis (PrEP) Outreach.

Kentucky Public Health Association (KPHA) Conference (April, 2019)

Covington, Kentucky, U.S.A

- Authors: Suur D. Ayangeakaa, Jelani Kerr, Ryan Combs, Lesley Harris, Jeanelle Sears, Toya Northington,Kimberly Parker, Emma Sterrett-Hong, and Karen Krigger.

- Poster Title: A Novel Approach to HIV Prevention: Examining Views of PreExposure Prophylaxis (PrEP)among Young African Americans in Louisville, KY. Adherence Conference Pre-session (2018)

Miami, Florida.

- Authors: Kerr, J., Golder, S., Crawford T., Ayangeakaa, S.

- Oral Title: Intersections of HIV-related and Incarceration Stigma among Formerly Incarcerated African Americans Living with HIV.

University of Louisville Graduate Student Regional Research Conference (2018) University of Louisville, Kentucky, U.S.A.

- Authors: Kelsey Burton, Suur D. Ayangeakaa, Jelani Kerr.

- Poster Title: Assessing the Factors Contributing to Sexual Concurrency and Increase in Sexual Partners Among African, Caribbean, and Black Women Living in Windsor, Ontario, Canada.

145th APHA Annual Meeting and Exposition (November 2017)

Atlanta, Georgia, U.S.A.

- Authors: Baraka Muvuka, Ryan Combs, Nida Ali, Suur D. Ayangeakaa.

- Oral Title: Developing Health Insurance Literacy Interventions in an Urban African American Community: Process, Lessons Learned, and Future Directions

144th APHA Annual Meeting and Exposition (November 2016)

Denver, Colorado, U.S.A.

- Authors: Baraka Muvuka, Aneshia Gray, Camila Aramburu, Rishtya M. Kakar, Suur D. Ayangeakaa, Katie F. Leslie, Karen W. Krigger, Cathy Spencer.

- Round Table Title: Increasing Access to HIV Pre-exposure Prophylaxis: An Assessment of Physician Barriers to Administration

Research! Louisville (October 2016)

University of Louisville, Kentucky, U.S.A.

- Authors: Suur D. Ayangeakaa, Ryan Combs, Baraka Muvuka, Nida Ali.

- Poster Title: Health Literacy in West Louisville: Examining The Development, Use, Applicability, And Design Of Health Insurance, Health Systems, And Health Behavior Literacy Materials To West Louisville Residents 
Research! Louisville (October 2016)

University of Louisville, Kentucky.

- Authors: Baraka Muvuka, Ryan Combs, Suur D. Ayangeakaa, Nida Ali, Monica Wendel, Trinidad Jackson.

- Poster Title: Factors Contributing to Inadequate Health Literacy among African Americans Residents

Research! Louisville (October 2016)

University of Louisville, Kentucky, U.S.A.

- Authors: Nida Ali, Ryan Combs, Baraka Muvuka, Suur D. Ayangeakaa, Monica Wendel.

- Poster Title: Promoting Health Through the Utilization of Novel Community-Based Participatory Research Methods: Using Boot Camp Translation to Improve Depression Literacy in West Louisville Residents

Research! Louisville (October 2015)

University of Louisville, Kentucky, U.S.A.

- Authors: Whitney Rogers, Beth, Young, Chandre' Chaney, Gaberiel Jones, Suur D. Ayangeakaa, Baraka Muvuka, Scott LaJoie.

- Poster Title: Redesigned medication labels better communicate information to patients

142nd APHA Annual Meeting and Exposition (November 2014)

New Orleans, Louisiana, U.S.A.

- Authors: Suur D. Ayangeakaa, Donna Sinclair.

- Poster Title: Emergency Room Integrated Routine HIV Screening: An innovative way to reach the major drivers of the epidemic - the undiagnosed

\section{PUBLICATIONS/SUMMITTED ARTICLES}

1. Nida M. Ali, Ryan M. Combs, Baraka Muvuka, Suur D. Ayangeakaa (2018). Addressing Health Insurance Literacy Gaps in an Urban African American Population: A Qualitative Study. Community Health. https://doi.org/10.1007/s10900-018-0541-x

2. Kelsey Burton, Suur Ayangeakaa, Jelani Kerr, Sarah Kershner, Eleanor Maticka-Tyndale (2018). Examining sexual concurrency and number of partners among African, Caribbean, and Black Women using the Social Ecological Model: Results from the ACBY study. The Canadian Journal of Human Sexuality 28 (1),46-56.

3. Baraka Muvuka, Ryan Combs, Suur D. Ayangeakaa, Nida Ali Monica Wendel, Trinidad Jeackson: Health Literacy in African American Communities. Barriers and Strategies Health Literacy Research and Practice (HLRP). Accepted 2019 (In Press). 
4. Suur D. Ayangeakaa, Baraka Muvuka, Aneshia Gray, Camila Aramburu, Rishtya M. Kakar, Katie F. Leslie, Karen W. Krigger, Cathy Spencer. Increasing Access to HIV Pre-Exposure Prophylaxis: An Assessment of Physician Barriers to Administration. In Preparation.

5. Suur D. Ayangeakaa, Jelani Kerr, Ryan Combs, Lesley Harris, Jeanelle Sears, Toya Northington, Kimberly Parker, Emma Sterrett-Hong, and Karen Krigger. Understanding intra-personal factors impacting pre-exposure prophylaxis (PrEP) engagement among young African-Americans. In Preparation.

6. Suur D. Ayangeakaa, Jelani Kerr, Ryan Combs, Lesley Harris, Jeanelle Sears, Toya Northington, Kimberly Parker, Emma Sterrett-Hong, and Karen Krigger. Beyond The Individual: Exploring Sociocultural and Structural-level Influences on HIV Pre-Exposure Prophylaxis Engagement among Young African Americans. In Preparation.

7. Suur D. Ayangeakaa, Jelani Kerr, Ryan Combs, Lesley Harris, Jeanelle Sears, Kimberly Parker. Serving the Underserved: A Qualitative Study Exploring Challenges of AIDS Service Organizations Conducting HIV Pre-Exposure Prophylaxis Outreach with African Americans. Abstract under review, APHA 2020

8. Suur D. Ayangeakaa, Jelani Kerr, Ryan Combs, Lesley Harris, Jeanelle Sears, Kimberly Parker. Become One with the Community: A Grounded Theory Study of AIDS Service Organizations' HIV Pre-Exposure Prophylaxis Strategies for African Americans. Abstract under review, APHA 2020

\section{GRANTS AND AWARDS}

Delta Omega Honorary Society in Public Health, Beta Pi Chapter (May, 2020)

\section{American Public Health Association}

- APHA Policy Action Institute Scholarship Award (February, 2020)

\section{University of Louisville}

- Non-Resident Tuition Differential Award (Fall 2016 - Spring 2017) \$6000.00 per semester

- Research! Louisville, Excellence in Health Disparities [Research Award - 2nd Place] (2016)

- Health Promotion Behavioral Sciences Tuition Scholarship Award (Spring \& Summer 2017) \$6000.00 per semester

- School of Public Health and Information Sciences Travel Fund (September 2019): $\$ 400$

- School of Public Health and Information Sciences Travel Fund (March 2019): \$500

- Graduate Student Council Travel Funds ( June 2019): \$200

- Graduate Merit Scholarship ( June 2019): \$115

\section{Chicago State University}


- Master of Public Health Graduate Division Award (2013)

- International Student Ambassador Award for Academic Excellence (2013)

- Global Ambassador Award for Academic Excellence (2012)

- University Honor Roll (2012 - 2013)

\section{Dordt College}

- Academic Honors Scholarship (2006 - 2009)

- Summer Ministries Scholarship (2006)

- International Students Grant (2006 - 2009)

\section{FUNDED RESEARCH AND CONTRACTS}

Project Title: West Louisville Health Literacy Project.

Funding Period: 2015-2018

- Agency: Funded by KentuckyOne Health through the Commonwealth Institute of Kentucky

- Amount: $\$ 300,000.00$

- Role: Research Associate

Project Title: Increasing Pre-exposure Prophylaxis among High-risk African Americans in Louisville, KY.

Funding Period: 2018-2020

- Agency: Funded by Jewish Heritage Fund for Excellence

- Amount: \$219,036.00

- Role: Research Associate

\section{WORK EXPERIENCE}

Emergency Department Health Counselor (2013)

Institution: Advocate Trinity Hospital, Chicago, Illinois, U.S.A

Project: HIV/Hepatitis C Prevention and Education.

- Rapid HIV testing, pre and post-test counseling, and prevention education in the emergency room to underserved populations - LGBT, African Americans, and Hispanics in South Chicago.

- Collected and reported risk assessment data.

- Prepared HIV positive clients for linkage to care.

Microbiology Laboratory Technician (2009 - 2011)

Organization: Well Pet, LLC. Mishawaka, Indiana, U.S.A

- Performed microbial testing using polymerase chain reaction.

- Conducted mycotoxin testing (mainly aflatoxin and deoxynivalenol-DON).

- Completed qualitative chemical analysis on pet food samples using near infrared (NIR).

- Performed quality control duties for final product release.

Quality Control Laboratory Assistant (2006 - 2009)

Organization: Sioux Biochemical Inc. Sioux Center, Iowa, U.S.A 
- Performed biochemical assays using standard operating procedures (SOPs).

- Extensive microbiology or bio burden assays.

- Performed assays on research proteins (i.e. trypsin and chymotrypsin activities).

- Performed assays using high pressure liquid chromatography (HPLC), electrophoresis, infrared spectroscopy, optical rotation and UV/visible spectrophotometry; tested for protein, chloride, sulfate and sodium limits in samples of chondroitin sulfate; tested for salmonella, e-coli, yeast, mold and staphylococcus aureaus in final product.

- Performed quality control duties for final product release.

\section{INTERNSHIPS}

Accreditation Coordination Specialist (2013 - 2014)

Office of Performance Management, Illinois Department of Public Health, Chicago, Illinois.

- Assisted the Health Department in preparing for accreditation through the Public Health Accreditation Board (PHAB).

- Researched, created, compiled, and reviewed documentation to meet PHAB standards and measures.

- Spearheaded the process for developing a quality improvement (QI) plan for the agency.

- Created, distributed and analyzed a QI survey to assess the QI culture of the agency.

- Reviewed grants and edited the agency Electronic Grants Application Management System.

Health Counselor (2012 - 2013)

Advocate Trinity Hospital, Chicago, Illinois, U.S.A.

- HIV and Hepatitis C counseling and testing in the emergency department.

- Collected and reported risk assessment data.

- Executed seven community outreach events along with other health counselors in schools, faith-based organizations, and the Chicago park district.

- Collaborated with Chicago Department of Public Health in conducting STI prevention outreach to Chicago public schools:

- Provided health promotion and disease prevention education to Chicago public schools and provided as-needed counseling.

○ Collected specimen for gonorrhea and chlamydia testing, handed off to on-site lab personnel for specimen preparation; ensured accuracy of participant demographic data.

- Performed in-office data entry of risk assessment results and questionnaire responses.

- Compiled quarterly reports for distribution to Chicago Department of Public Health.

Health Assistant $\quad$ (Summer, 2008)

Saint Luke Hospital, Kasei, Ghana. 
- Assisted in out-patient department (OPD), measured patients vital signs including blood pressure, temperature, height, and weight.

- Assisted in dispensary, provided guidance to patients regarding prescription medication adherence.

- Shadowed surgeon in operating room (hernia surgery).

Orphan Missions Assistant (Summer, 2006)

Every Orphans Hope, Lusaka, Zambia.

- Supervised, for eight hours daily, six orphans (ages 3-11).

- Taught math and reading to children orphaned by HIV/AIDS.

- Planned and conducted two summer vacation Bible camps for children orphaned by HIV/AIDS.

- Supervised 15-20 kids in each camp, helped camp participants practice songs, dance, sports, and crafts.

- Updated orphan sponsorship profiles; created orphan sponsorship awareness.

- Collaborated with local churches and communities to build bonds between orphans, their neighbors and pastors, and identify the neediest of orphans as candidates for sponsorship.

\section{PROFESSIONAL TRAINING/ CERTIFICATIONS}

\section{HIV/AIDS Counseling and Testing}

Provided by: Kentucky Department of Health, HIV/AIDS Branch

African American HIV Education and Prevention Instructor

Provided by: Chicago Department of Public Health

\section{HIV/AIDS Counseling and Testing}

Provided by: Midwest AIDS Training and Education Center (MATEC)

\section{Hepatitis Training}

Provided by: Chicago Department of Public Health

Polymerase Chain Reaction (PCR) Training

Provided by: Bio-Control Genetic Detection Systems

\section{PROFESSIONAL MEMBERSHIP}

Member - American Public Health Association (APHA) Since 2013

Member - American Academy of HIV Medicine (AAHIVM) Since 2016

Member - Kentucky Public Health Association (KPHA) Since 2019

\section{JOB SHADOW}

Dr. Richardson's Neighborhood Family Practice Oak Park, IL (2013-2014)

- Observed Family Practice physician (history-taking, patient examination, and consultation). 
- Assisted weekly in clinic for 3-4 hours

- Participated actively in weekly case studies, patient simulations, and problembased learning to generate hypotheses, formulate and test theories, and produce a diagnosis.

Sioux Land Medical Education Foundation, Sioux City, IA

Sioux Center Medical Clinic Sioux Center, IA $\quad$ (2007 - 2008)

- Observed an OBGYN Physician in family practice (history-taking and consultation).

- Shadowed surgeon in operating room (gall bladder removal and laparoscopic surgery).

\section{VOLUNTEER PROJECTS}

Shelter Monitor $\quad$ (2007 - 2009)

Family Crisis Center, Sioux Center, Iowa, U.S.A.

- Provided shelter residents with food, clothing and hygiene resources.

- Ensured safety of residents via monitoring entrances and exits and notifying police of suspicious activity or direct threats.

Missions Project Participant (2005 - 2006)

Dort College, Sioux Center, Iowa, U.S.A.

- Project: Center, Colorado: Assisted with roofing, plumbing and painting.

- Hurricane Rita Relief, Lumberton, TX: landscaping projects to help individuals return to their homes.

\section{COMMITTEES AND ORGANIZATION ENGAGEMENTS}

International Student Council, University of Louisville, Louisville KY

- Member (2019 - 2020)

Graduate Student Council (GSC), University of Louisville, Louisville KY

- GSC Representative - School of Public Health (2015 - 2017)

- Committee Member - GSC Advocacy and involvement committee

$(2015-2016)$

- Committee Member: GSC Research Fund committee (2016 - 2017)

Student Government Association - SPHIS University of Louisville, Louisville KY

- GSC Representative (2015 - 2017)

MPH Student Association, Chicago State University, Chicago, IL

- Vice President of Finance (2011- 2013)

Economic Recovery Institute, Inc. Sub-Saharan Africa

Project Manager: Empowerment program for widows, orphans, and vulnerable children (2011- 2013)

- Co-developed pilot project plan. 
- Developed the eligibility and selection criteria for program participants.

- Collected donations (clothing, shoes and non-perishable food items) to be shipped to Africa to aid the identified WOVC.

- Co-executed fund raisers for the agency for project sustenance.

Dort College, Sioux Center, IA

- Treasurer - Future Physicians Club, 2008

- Member - Women in Science and Engineering (WISE) 